\title{
Effects of Diameter-limit and Two-age Timber Harvesting on Songbird Populations on an Industrial Forest in Central West Virginia
}

\author{
Cathy Ann Weakland \\ West Virginia University
}

Follow this and additional works at: https://researchrepository.wvu.edu/etd

Part of the Forest Sciences Commons, and the Other Ecology and Evolutionary Biology Commons

\section{Recommended Citation}

Weakland, Cathy Ann, "Effects of Diameter-limit and Two-age Timber Harvesting on Songbird Populations on an Industrial Forest in Central West Virginia" (2000). Graduate Theses, Dissertations, and Problem Reports. 1243.

https://researchrepository.wvu.edu/etd/1243

This Dissertation is protected by copyright and/or related rights. It has been brought to you by the The Research Repository @ WVU with permission from the rights-holder(s). You are free to use this Dissertation in any way that is permitted by the copyright and related rights legislation that applies to your use. For other uses you must obtain permission from the rights-holder(s) directly, unless additional rights are indicated by a Creative Commons license in the record and/ or on the work itself. This Dissertation has been accepted for inclusion in WVU Graduate Theses, Dissertations, and Problem Reports collection by an authorized administrator of The Research Repository @ WVU.

For more information, please contact researchrepository@mail.wvu.edu. 
Effects of Diameter-limit and Two-age Timber Harvesting on

Songbird Populations on an Industrial Forest in Central West Virginia

\author{
Cathy A. Weakland
}

Dissertation submitted to the College of Agriculture, Forestry, and Consumer Sciences at West Virginia University in partial fulffillment of the requirements for the degree of

\title{
Doctor of Philosophy \\ in
}

Forest Resource Science:

Wildlife and Fisheries Resources

Petra B. Wood, Ph. D., Chair

Andrew F. Egan, Ph. D.

W. Mark Ford, Ph. D.

Linda S. Gribko, Ph. D.

Robert C. Whitmore, Ph. D.

Division of Forestry

Morgantown, West Virginia

2000

Keywords: Diameter-limit; Forest management; Landscape ecology; Songbird populations; Timber harvesting; Two-age harvest 


\title{
ABSTRACT \\ Effects of Diameter-limit and Two-age Timber Harvesting on Songbird Populations on an Industrial Forest in Central West Virginia
}

\author{
Cathy A. Weakland
}

Many studies examining forest fragmentation effects on songbirds have been conducted in landscapes significantly altered by urbanization or agriculturalization rather than forested landscapes. There is some evidence that forest fragmentation due to timber harvesting has different effects on bird abundance than fragmentation from other land uses. It is unknown how songbirds respond to different forms of timber harvesting as fragmentation events. Also, it is unclear if microhabitat-level or landscape-level characteristics are more important predictors of breeding bird occurrence in the central Appalachians. The objectives of my study were to determine the short-term effects of diameter-limit and two-age timber harvesting on the abundance and nest survival of songbirds and to determine the specific landscape-level and microhabitat-level features of a recently fragmented industrial forest that affect songbird abundance.

I examined songbird abundance at 50-m fixed-radius point counts and nest survival on eight 40-ha nest searching plots on the Westvaco Wildlife and Ecosystem Research Forest in Randolph County, West Virginia, 1-yr before and 1-2-yr after partial harvesting. Microhabitat variables were measured within each 50 -m radius point count plot, and landscape metrics were calculated within a $1-\mathrm{km}$ radius of each point count.

For most songbird species present prior to harvest, abundances changed little following diameter-limit harvesting. Interior-edge species and total abundance of songbirds were more abundant in diameter-limit harvested areas. The abundance of most songbird species present prior to two-age harvesting also changed little after the harvest. Interior-edge species had higher abundance in two-age harvested areas. Two species, the Dark eyed-Junco (Junco hyemalis) and Veery (Catharus fuscescens), were found more frequently in nonfragmented landscapes than in landscapes fragmented by regeneration (two-age and seed tree) harvests, and two different species, the Ovenbird (Seirus aurocapillus) and Scarlet Tanager (Piranga olivacea), were found more frequently in nonfragmented landscapes than in landscapes fragmented by diameter-limit harvests. However, these results might not apply to species that were rare or uncommon on the study area.

It appears that timber harvesting in this forested landscape is not having short-term deleterious impacts on most songbirds. These results could be used by land managers to maintain healthy and diverse populations of songbirds in extensively forested regions. 


\section{Dedicated to the memory of Lee Richards}

"Forgetting what is behind and straining toward what is ahead, I press on toward the goal to win the prize for which God has called me heavenward in Christ Jesus ... Only let us live up to what we have already obtained."

Phil. 3:13-14, 16

"If God in the final judgement would ask you a question about the Creation, what might that question be?... 'What did you do with my creation?' ... or 'How did I make the world?' ...Are we preparing ourselves and those we influence to answer 'How did I do it?' or 'What did you do with it?'” Calvin B. Dewitt 


\section{ACKNOWLEDGEMENTS}

This research was funded by Westvaco Corporation, Forest Resources Division, and the West Virginia Division of Natural Resources Nongame Wildlife and Natural Heritage Program. The West Virginia Cooperative Fish and Wildlife Research Unit, USGS, BRD, provided equipment and logistical support. I owe special thanks to my advisor, Petra Wood, for her continual support and suggestions during the entire length of this project. Petra, you're the best, and I admire your dedication and hard work. I would like to thank my committee members, Robert Whitmore, Linda Gribko, Mark Ford, and Andy Egan who gave me advice and encouragement throughout my time at WVU. Mark Ford, Sydney McIntyre, Sarah Clapham, Rick Odum, and Sally Lane of Westvaco provided technical and/or field assistance. Additionally, I am forever indebted to Ann Steketee for her advice and consultation on GIS and Fragstats analyses.

I would also like to thank the many field technicians who assisted with nest searching, point count surveys, and habitat sampling: Dave Jones, Brian Lindley, Cheryl Allen, Travis Moulder, Paul Callo, Rhonda Wuensch, Becky Murray, Brett Magdasy, Wyatt Nimitz, Aaron Brady, Mike Sorice, Jayme Waldron, Steve Cundy, Lisa Kendall, and Dennis Feeney. You had to survive snow storms in May, flash flooding, countless thunderstorms, fighting through logging debris and rhododendron, rising at 4:30am, hiking in difficult terrain, delayed paychecks, skunks in the kitchen, and living with 20+ people. Thank you for your hard work and for surviving without complaining too much. The following graduate students also provided support and encouragement: Gary Williams, Jeff Duguay, Pam Denmon, Jerry and Ann Steketee, Dorothy Tinkler, Ron Huffman, Nikki and Steven Castleberry, Cheryl Allen, Rebecca Smith, Jon Zuck, and J. B. Churchhill. A huge thank-you also goes to Janine Ostergren and Becky Nestor for the joy and energy that they brought to my life and their willingness to give me aid me whenever I needed it. You are the best secretaries ever, and I'm sorry for keeping you from your work with all those chat sessions (well, okay, maybe I'm not sorry).

I would like to thank my family for their love and support throughout my studies. Mom and Dad, you are the best parents anyone could ever have. Thank you for instilling a strong work ethic in me and a desire to achieve great things. I would also like to thank members of my Christian family, especially Lisa Arroyo, Richard Webster, Alan Batchelor, Sandy and Susan 
Bigelow, Sean and Amy Wightman, members of the Christian Student Fellowship and Intervarsity Christian Fellowship, Covenant Evangelical Methodist Church, and the "Percival Prayer" warriors. You kept me focused on Christ and the truly important things in life and stretched my way of thinking and worshipping. You exemplify by your faith, love, and actions what it means to be a true witnessing community shining the light of Christ to the world. Keep praying for WVU and Morgantown; the battle may last long and the clouds may be dark, but Jesus has already won the victory and His light shines upon you.

Lastly, but most importantly, I want to thank my Lord and Savior Jesus Christ for His countless blessings. Your unconditional love and acceptance changed me forever and gave my life purpose and meaning, and it will take eternity for me to express the depth of my gratitude and devotion. 


\section{TABLE OF CONTENTS}

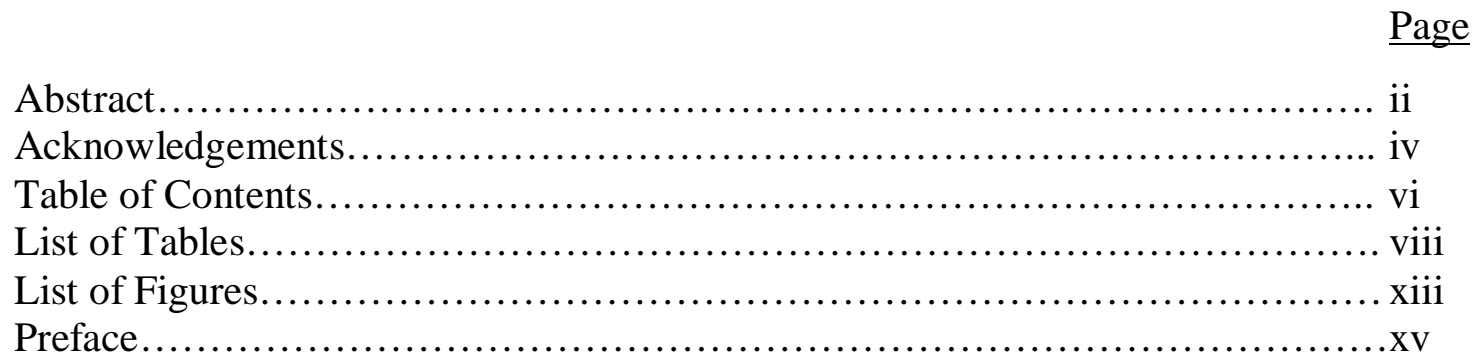

\section{CHAPTER 1: SONGBIRD POPULATIONS IN AN INDUSTRIAL FOREST}

LANDSCAPE IN CENTRAL WEST VIRGINIA

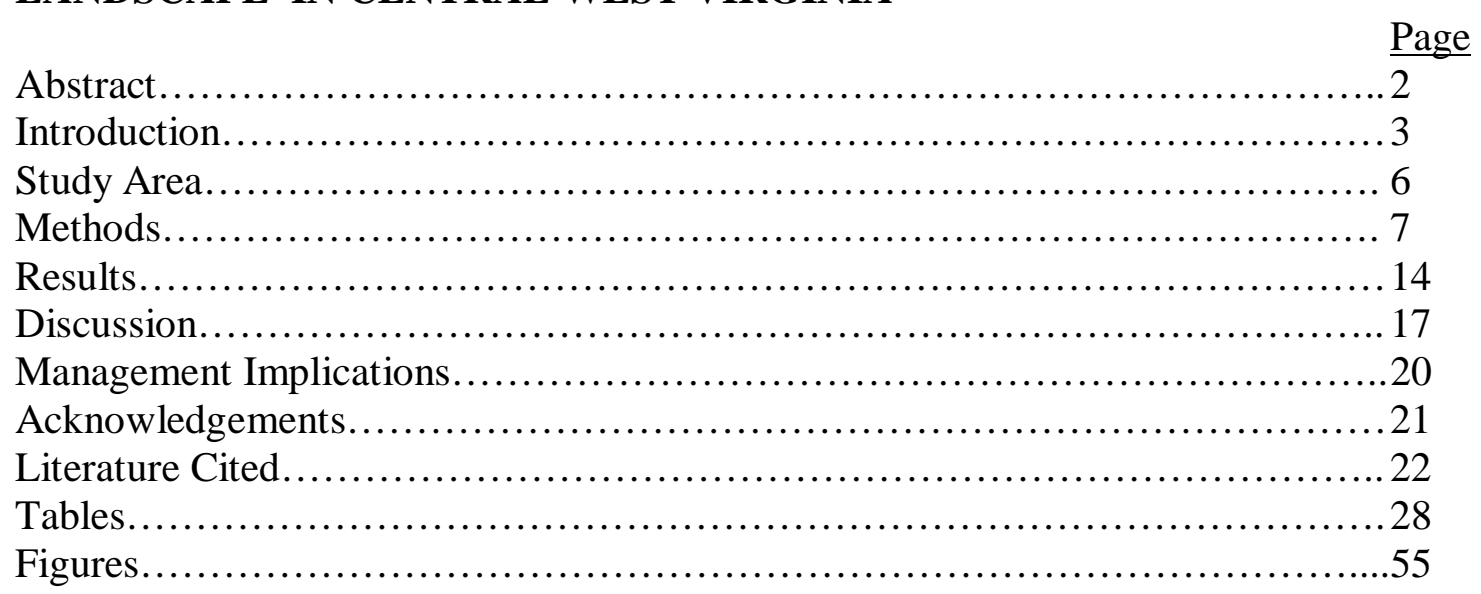

\section{CHAPTER 2: RESPONSES OF SONGBIRDS TO DIAMETER-LIMIT CUTTING IN THE CENTRAL APPALACHIANS OF WEST VIRGINIA}

\begin{tabular}{|c|c|}
\hline & $\frac{\text { Page }}{57}$ \\
\hline Introduction... & .57 \\
\hline Methods....... & .59 \\
\hline Results..... &. .64 \\
\hline Discussion. &. .66 \\
\hline Conclusions........... &. .69 \\
\hline Acknowledgements... & .69 \\
\hline Literature Cited....... &. .70 \\
\hline Tables.................. &. .74 \\
\hline Figures.............. & $\ldots 82$ \\
\hline
\end{tabular}


CHAPTER 3: SHORT-TERM EFFECTS OF TWO-AGE HARVESTING ON SONGBIRD ABUNDANCE AND NESTING SUCCESS

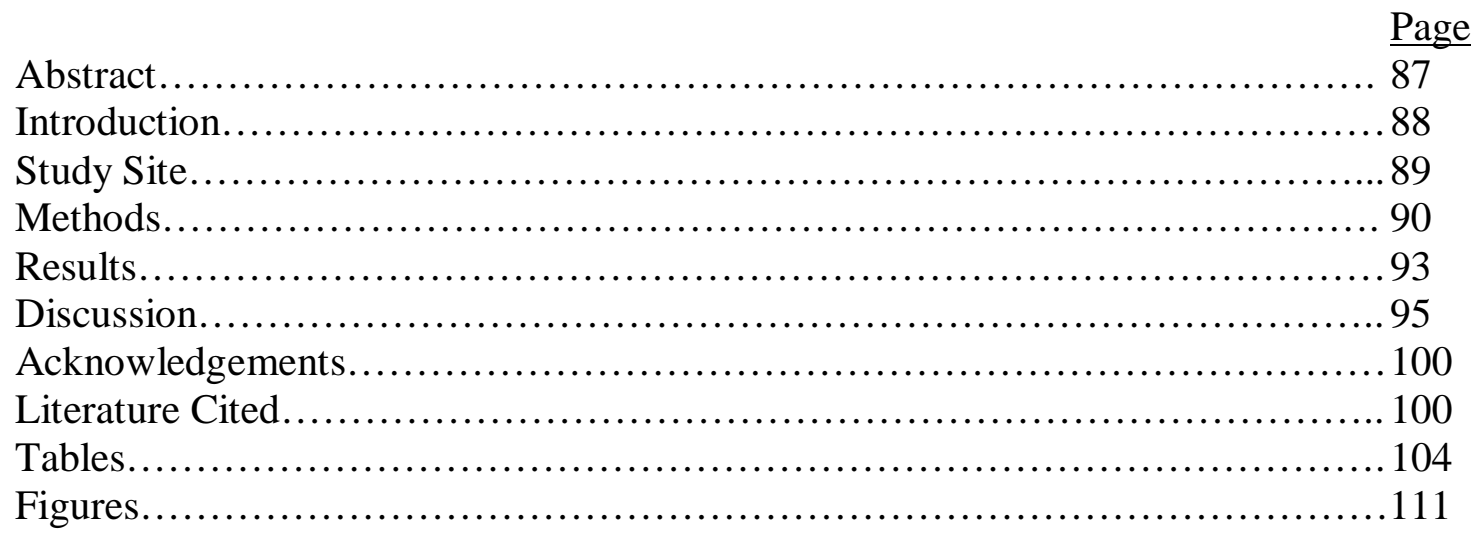

\section{CHAPTER 4: LANDSCAPE AND MICROHABITAT FACTORS INFLUENCING SONGBIRD OCCURRENCE ON AN INDUSTRIAL FOREST IN CENTRAL WEST VIRGINIA}

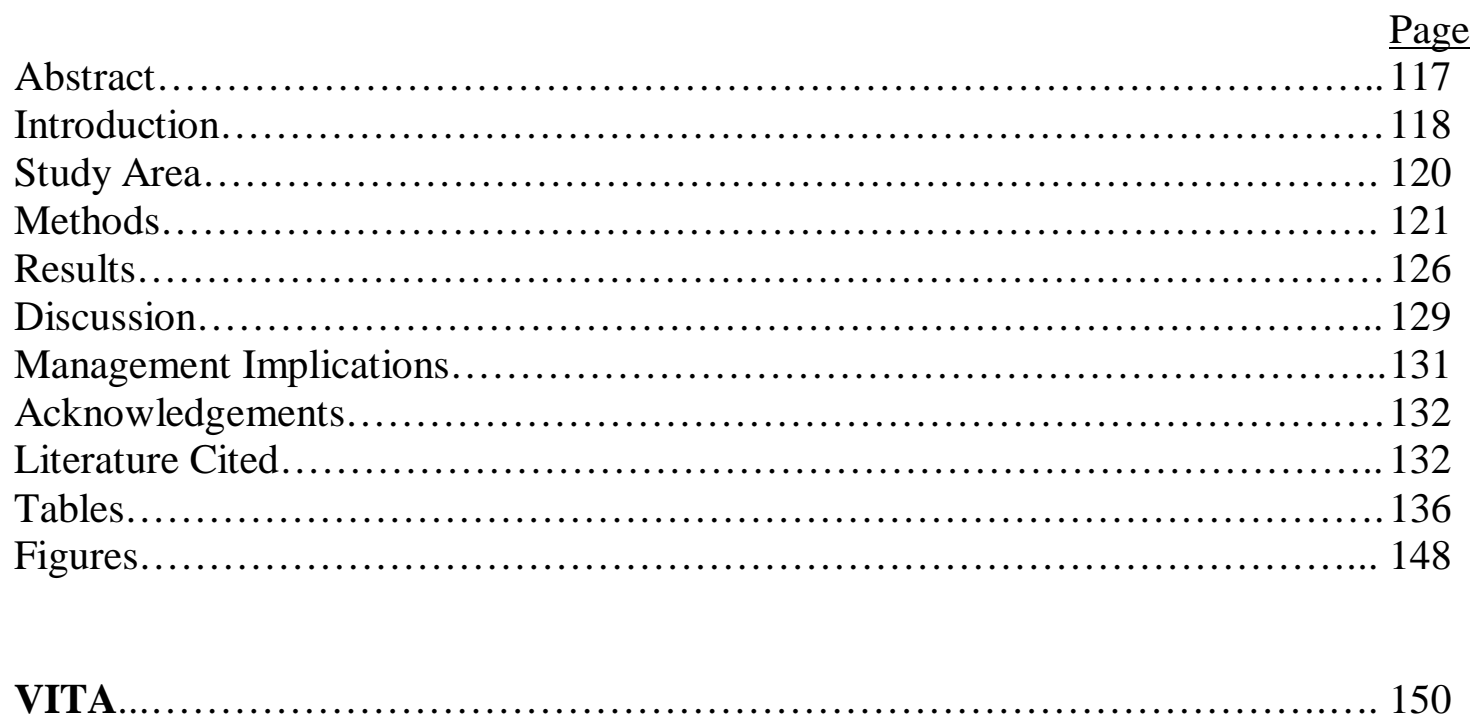




\section{LIST OF TABLES}

\section{Chapter 1:}

Table 1.1. Weather and sky code indices used during point count surveys on the

Westvaco Wildlife and Ecosystem Research Forest, Randolph County, West Virginia, 1996-1998.

Table 1.2. Common names, scientific names, habitat groups, nesting guilds, and migratory strategies of songbird species observed on the Westvaco Wildlife and Ecosystem Research Forest, Randolph County, West Virginia, 1996-1998.

Table 1.3. Landtype classification developed from Westvaco FRIS ${ }^{\mathrm{TM}}$ data, and landtype groupings for this study, on the Westvaco Wildlife and Ecosystem Research Forest, Randolph County, West Virginia

Table 1.4 . Calculation of the vertical structure index (VSI) used in ANCOVA models comparing songbird abundance among forest types on the Westvaco Wildlife and Ecosystem Research Forest, Randolph County, West Virginia, 1996-1998

Table 1.5. Raptor and gamebird species observed on the Westvaco Wildlife and Ecosystem Research Forest, Randolph County, West Virginia, 1996-1998.

Table 1.6. Mean abundance per point count, standard error (SE), and 95\% confidence intervals for total songbirds, habitat groups, nesting guilds, and species on the Westvaco Wildlife and Ecosystem Research Forest, Randolph County, West Virginia, 1996-1998... 35

Table 1.7. Total abundance and percent frequency of occurrence of total songbirds, habitat groups, nesting guilds, and species on the Westvaco Wildlife and Ecosystem Research Forest, Randolph County, West Virginia, 1996-1998.

Table 1.8. ANOVA and Scheffe' multiple comparison test results comparing songbird abundance of species observed at $\geq 20 \%$ of point counts among years on the Westvaco Wildlife and Ecosystem Research Forest, Randolph County, West Virginia , 1996-1998. Years with the same letter do not differ at the family alpha level of 0.10 . "A" indicates highest abundance, followed by "B"and " $\mathrm{C}$ "

Table 1.9. Means and standard errors of total songbird abundance, abundance of habitat groups and nesting guilds, and of individual species observed at $\geq 20 \%$ of point counts on submesic $(n=25)$, mesic $(n=29)$, and xeric slopes $(n=17)$, and ridges $(n=39)$ at unharvested point counts on the Westvaco Wildlife and Ecosystem Research Forest, Randolph County, West Virginia, 1996-1998. Means are for untransformed data. 
Table 1.10. ANOVA and Scheffe' multiple comparison test results comparing songbird abundance (species at $\geq 20 \%$ of point counts) among landtypes on the Westvaco Wildlife and Ecosystem Research Forest, Randolph County, West Virginia , 1997-1998 (unharvested point counts only). Landtypes with the same letter do not differ at the family alpha level of 0.10 . "A" indicates highest abundance, followed by "B", "C", and "D"

Table 1.11. Means and standard errors for total abundance, abundance of habitat groups and nesting guilds, and abundance of individual species (at $\geq 20 \%$ of point counts) by mature mixed woods (1996: $n=48 ; 1997: n=33 ; 98: n=30)$, mature hardwoods (1996: $n=96$; 1997: $n=84 ; 1998: n=80)$, diameter-limit harvests (1997: $n=17 ; 1998: n=22)$, and two-age harvests (1997-98: n=10) on the Westvaco Wildlife and Ecosystem Research Forest, Randolph County, West Virginia. Means are for untransformed data.................. 46

Table 1.12. ANCOVA and Scheffe' multiple comparison test results comparing songbird abundances (species at $\geq 20 \%$ of point counts) among forest habitats on the Westvaco Wildlife and Ecosystem Research Forest, Randolph County, West Virginia, 1997-1998. Habitats with the same letter do not differ at the family alpha level of 0.10 . "A" indicates highest abundance, followed by "B", "C", and "D" ...

Table 1.13. Number of nests, exposure days, daily survival, and total survival for songbird nests by treatment found on the Westvaco Wildlife and Ecosystem Research Forest, Randolph County, West Virginia, 1996-1998.............................. 50

Table 1.14. Means, standard errors (SE), and 95\% confidence intervals (CI) for habitat variables surrounding successful $(n=90)$ and predated nests $(n=95)$ on the Westvaco Wildlife and Ecosystem Research Forest, Randolph County, West Virginia, 19961998.

\section{Chapter 2:}

Table 2.1. Means and standard errors (SE) for the abundance of nesting guilds and habitat groups at harvested, peripheral, and unharvested stands the breeding season before harvest (1996) and two breeding seasons after (1997-98) on the Westvaco Wildlife and Ecosystem Research Forest, Randolph County, West Virginia. Means are for untransformed data and are not adjusted for other variables in ANCOVA models......74

Table 2.2. Means and standard errors (SE) for songbird abundance (species at $\geq 20 \%$ of point counts) at harvested, peripheral, and unharvested stands the breeding season before (1996) harvest and two breeding seasons after (1997-98) on the Westvaco Wildlife and Ecosystem Research Forest, Randolph County, West Virginia. Means are for untransformed data and are not adjusted for other variables in ANCOVA models. 
Table 2.3. Species for which nests were located on unharvested and pre-harvest treatment areas in 1996 and on unharvested (unhar.), peripheral (per.), and harvested (har.) areas on the Westvaco Wildlife and Ecosystem Research Forest, Randolph County, West Virginia, 1997-1998.................................................. 79

Table 2.4. Mayfield daily nest survival estimates, exposure days, and total survival for nesting guilds and for total nests in unharvested, harvested, and peripheral areas on the Westvaco Wildlife and Ecosystem Research Forest, Randolph County, West Virginia, 1996-1998. Daily and total survival were calculated as the product of survival during the incubating and brooding periods

Table 2.5. Comparison of habitat variables in stands harvested using the diameter-limit method and on unharvested plots on the Westvaco Wildlife and Ecosystem Research Forest, Randolph County, West Virginia in 1998. Means are for untransformed data.

Table 2.6. Comparison of species-specific responses to partial harvesting methods in different regions of the United States....

\section{Chapter 3:}

Table 3.1. Songbird species for which nests were located on unharvested and pre-harvest treatment areas in 1996 and on unharvested (unhar.), peripheral (per.), and harvested (har.) areas in 1997-1998 on the Westvaco Wildlife and Ecosystem Research Forest, Randolph County, West Virginia.

Table 3.2. Mayfield daily nest survival estimates, exposure days, and total survival for nesting habitat groups and total nests at unharvested, harvested, and peripheral plots on the Westvaco Wildlife and Ecosystem Research Forest, Randolph County, West Virginia 1996-98.

Table 3.3. Means and standard errors (SE) for songbird abundance at harvested, peripheral, and unharvested stands one year before (1996) harvest and two-years after (1997-98) on the Westvaco Wildlife and Ecosystem Research Forest, Randolph County, West Virginia. Means are for untransformed data and are not adjusted for other terms in the model

Table 3.4. Means and standard errors (SE) for the abundance of nesting guilds and habitat groups at harvested, peripheral, and unharvested stands one year before harvest (1996) and two years after (1997-98) on the Westvaco Wildlife and Ecosystem Research Forest, Randolph County, West Virginia. Means are for untransformed data and are not adjusted for other terms in the model. 
Table 3.5. Means and standard errors for habitat measurements on two-age harvested stands and on unharvested stands on the Westvaco Wildlife and Ecosystem Research Forest, Randolph County, West Virginia, 1998. Means are for untransformed data......

\section{Chapter 4:}

Table 4.1. Weights used to calculate the contrast-weighted edge density among all possible pairs of edges on the Westvaco Wildlife and Ecosystem Research Forest, Randolph County, West Virginia, 1996-1998

Table 4.2. Presence of songbird species in fragmented $(n=212)$ and nonfragmented $(n=72)$ landscapes on the Westvaco Wildlife and Ecosystem Research Forest, Randolph County, West Virginia, 1996-1998.

Table 4.3. Presence of songbird species in landscapes fragmented by diameter-limit harvests $(n=105)$, and by regeneration harvests $(n=19)$, and in nonfragmented $(n=159)$ landscapes on the Westvaco Wildlife and Ecosystem Research Forest, Randolph County, West Virginia, 1996-1998. Within a species, proportions with the same letter do not differ (pairwise Cochran-Mantel-Haenszel Chi-square, $\mathrm{P} \leq 0.03$ ).

Table 4.4. Means, standard errors (SE), minimum (Min) and maximum values (Max), and $95 \%$ confidence coefficients (CI) for landscape variables on the Westvaco Wildlife and Ecosystem Research Forest, Randolph County, West Virginia , 1996-1998. Means with different letters differ among years (ANOVA, $\mathrm{P} \leq 0.10$ ). Means are for untransformed

data

Table 4.5. Significant landscape variables selected by stepwise logistic regression for predicting the presence of songbird species on the Westvaco Wildlife and Ecosystem Research Forest, Randolph County, West Virginia, 1996-1998.

Table 4.6. Means, standard errors (SE), minimum (Min) and maximum values (Max), and $95 \%$ confidence coefficients (CI) for microhabitat and landscape variables used in microhabitat and combined logistic regression models on the Westvaco Wildlife and Ecosystem Research Forest, Randolph County, West Virginia, 19961998. Means are for untransformed data.

Table 4.7. Microhabitat variables selected by stepwise logistic regression to predict the presence of interior-edge and forest-interior species on the Westvaco Wildlife and Ecosystem Research Forest, Randolph County, West Virginia, 19961998. 
Table 4.8. Combined landscape and microhabitat variables chosen by stepwise logistic regression to predict the presence of songbirds in 1996 and 1998 on the Westvaco Wildlife and Ecosystem Research Forest, Randolph County, West Virginia $(\mathrm{n}=69$ each year)

Table 4.9. Best-fit models predicting songbird occurrence on the Westvaco Wildlife and Ecosystem Research Forest, Randolph County, West Virginia, 1996-1998. Models with better performance are marked with an " $\mathrm{X}$ "

Table 4.10. Wald chi-square test statistics and significance levels for variables selected in combined landscape and microhabitat models predicting bird species occurrences on the Westvaco Wildlife and Ecosystem Research Forest, Randolph County, West Virginia, 1996-1998. 


\section{LIST OF FIGURES}

\section{Chapter 1:}

Figure 1. 1. Location of the Westvaco Wildlife and Ecosystem Research Forest, in

Figure 2. 2. Forest types on the Westvaco Wildlife and Ecosystem Research Forest, Randolph County, West Virginia in a) 1996 and b) 1998

Figure 1. 3. Location of nest plots and point count plots on the Westvaco Wildlife and Ecosystem Research Forest, Randolph County, West Virginia, 1996-1998. ...

Figure 1.4. Mean abundance of total songbirds, habitat groups, and nesting guilds on the Westvaco Wildlife and Ecosystem Research Forest, Randolph County, West Virginia, 1996-1998. Bars within each group/guild with the same letter do not differ (Scheffe Multiple Comparison Test, family $\mathrm{P} \leq 0.10)$. Means are for untransformed data.

\section{Chapter 2:}

Figure 2.1. Layout of nest searching plots, point count plots, and habitat sampling subplots on the Westvaco Wildlife and Ecosystem Research Forest, Randolph County,

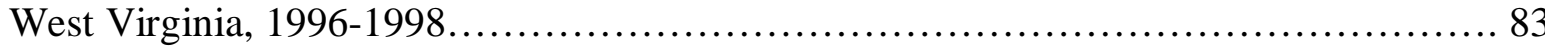

Figure 2.2. Mean bird abundance in harvested, peripheral, and unharvested stands on the Westvaco Wildlife and Ecosystem Research Forest, Randolph County, West Virginia, 1997-1998. Error bars represent \pm 1 standard error. Means are for untransformed data.

Figure 2.3. a) Mean density of woody vegetation in nine size classes and b) percent cover in six canopy layer classes and four ground cover classes before (1996) and after diameterlimit harvesting (1998) on the Westvaco Wildlife and Ecosystem Research Forest, Randolph County, West Virginia. Error bars represent \pm 1 standard error. Bars with a '*' differ at $\mathrm{P} \leq 0.10$. Means are for untransformed data.

\section{Chapter 3:}

Figure 3.1. Layout-out of nest searching plot, point count plots, and habitat sampling subplots on the Westvaco Wildlife and Ecosystem Research Forest, Randolph County, West Virginia, 1996-1998.

Figure 3.2. Mean total abundance of songbirds at harvested, peripheral, and intact (unharvested) stands on the Westvaco Wildlife and Ecosystem Research Forest, Randolph County, West Virginia, 1997-98. Bars with different letters differ at $\mathrm{P} \leq 0.10 \ldots \ldots 112$ 
Figure 3.3. Mean density of trees in five size classes one growing season before (1996) and two growing seasons after harvesting (1998) on two-age stands on the Westvaco Wildlife and Ecosystem Research Forest, Randolph County, West Virginia. Error bars represent \pm 1 standard error. Bars with an asterisk differ before and after harvesting at $\mathrm{P} \leq 0.10$. Means are for untransformed data.

Figure 3.4. Mean density of shrubs, saplings, and one growing season before (1996) and two growing seasons after harvesting (1998) on two-age stands on the Westvaco Wildlife and Ecosystem Research Forest. Error bars represent \pm 1 standard error. Bars with an asterisk differ before and after harvesting at $\mathrm{P} \leq 0.10$. Means are for untransformed data. ...

Figure 3.5. Mean percent cover in six canopy layer classes and four ground cover classes one growing season before (1996) and two growing seasons after harvesting (1998) on two-age stands on the Westvaco Wildlife and Ecosystem Research Forest, Randolph County, West Virginia. Error bars represent \pm 1 standard error. Bars with an asterisk differ before and after at $\mathrm{P} \leq 0.10$. Means are for untransformed data.

\section{Chapter 4:}

Figure 4.1. Landscape composition of the Westvaco Wildlife and Ecosystem Research Forest, Randolph County, West Virginia in a) 1996 and b) 1998

Figure 4.2. Layout-out of point count plots and habitat sampling subplots on the Westvaco Wildlife and Ecosystem Research Forest, Randolph County, West Virginia, 1996-1998. 


\section{PREFACE}

This dissertation has been written in the form of 4 chapters. The first chapter gives an overview of songbird populations on an industrial forest prior to and 1- and 2-years following large-scale harvesting. The second chapter focuses on the immediate effects of diameter-limit harvesting on songbird abundance and nesting success, and the third chapter focuses on the effects of two-age harvesting on songbird abundance and nesting success. The fourth chapter examines the effects of landscape parameters (fragmentation) and microhabitat parameters on songbird abundance. Chapter 1 is an overview and will be submitted to Westvaco Corporation as part of a final report and is formatted following the style of the Journal of Wildlife Management. The last 3 chapters will be revised for submission to the following journals:

Chapter 2 - Forest Ecology and Management

Chapter 3 - Journal of Field Ornithology

Chapter 4 - Journal of Wildlife Management 
CHAPTER 1

SONGBIRD POPULATIONS IN AN INDUSTRIAL FOREST LANDSCAPE IN CENTRAL WEST VIRGINIA 


\begin{abstract}
Management of private forest lands in the East is vitally important to the conservation of songbird species because private forests dominate the eastern deciduous forest. Many songbird species experiencing declines elsewhere are remaining stable or increasing in West Virginia, and private landowners own approximately $90 \%$ of the state's forested lands. The purpose of this study was to examine the immediate impacts of forest management practices on an industrial forest on songbird abundance and nest survival, and to examine abundance among landtypes. The study area was the 3360-ha Westvaco Wildlife and Ecosystem Research Forest (WWERF) in Randolph County, West Virginia. I examined songbird abundance throughout the forest and nesting success on eight 40-ha plots in 1996 and in 1997-98 after several diameter-limit and regeneration (seed tree and two-age) harvests were completed. The abundance of some species and of canopy-nesting and forest-interior species declined as more stands were harvested on the WWERF, while interior-edge species responded positively to harvesting. Some species also showed preferences for certain landtypes and forest habitats, but results could have been confounded by the high density of white-tailed deer (Odocoileus virginianus) on the forest. Overall nest survival appeared to be lower on the forest than in other areas of West Virginia, possibly because of the high amount of edge habitat on the WWERF. The density of deer should be reduced to increase understory vegetation for ground, shrub, and subcanopy nesting species. Foresters should balance age classes across the forest and increase rotation lengths to provide habitat for both early successional and forest interior species.
\end{abstract}

Key words: industrial forestry, nest survival, songbirds, timber harvest, white-tailed deer 


\section{INTRODUCTION}

Over the last 2 decades the Breeding Bird Survey has shown that populations of some neotropical migratory songbirds are declining, especially in the eastern United States (Peterjohn et al. 1995). Although some contend that evidence supporting the existence of declines is weak (James and McCulloch 1995), most agree that attempts should be made to conserve populations if possible. Causes of these declines are not well understood, but habitat loss and modification in both the temperate zone and the tropics are suspected to be at least partially responsible (Finch 1991, Hagan and Johnston 1992). Different factors are likely affecting population sizes in different regions of North America and/or the tropics (O’Connor 1992). For example, in severely fragmented forest regions of the Midwest songbird populations may be experiencing declines due to high levels of nest parasitism by the brown-headed cowbird (Molothrus ater) (Robinson et al. 1995). However, in some highly forested regions of the East cowbird parasitism is much less severe and likely contributes little to population declines (Hagan et al. 1997, Demeo 1999, Duguay et al. in press).

Forest fragmentation on the breeding grounds commonly has been suggested to be a major factor influencing population declines (Finch 1991, Robinson et al. 1995), and much research over the last 15 years has focused on the effects of forest fragmentation on songbird abundance and nesting success (Faaborg et al. 1995). Fragmentation is defined in simplest terms by Lord and Norton (1990) as " the disruption of continuity." Therefore, fragmentation can have many forms, from permanent conversion of forests to agricultural fields to disruption of mature forests by timber harvesting. Research on the effects of fragmentation on songbirds typically has been conducted in permanently fragmented landscapes where a large tract of forest is converted to another land use and only small, isolated forest stands remain (Faaborg et al. 1995). Less research has focused on the effects of temporary fragmentation - such as timber harvesting in large forest tracts (Hagan et al. 1996).

Fragmentation results in a loss of habitat that is both qualitative and quantitative for species that were present before the disturbance. The loss is quantitative because the area of forest, and therefore the habitat available, has been reduced, but it is also qualitative because the habitat that remains may be less suitable for nesting because of increases in edge habitat and interspecific competition (Finch 1991, Faaborg et al. 1995). Increases in nest predation and parasitism are associated with increases in edge habitat in fragmented landscapes (Gates and 
Gysel 1978, Brittingham and Temple 1983). However, Hartley and Hunter (1998) suggest that these edge effects are not significant in landscapes that are heavily forested.

In simplest terms, a landscape is a mosaic of heterogenous patches of habitat where species live and disperse (Dunning et al. 1995, McGarigal and Marks 1995). In extensively forested regions the mosaic is composed of different forest seres, whereas in permanently fragmented regions the mosaic is composed of several distinct patches, such as young and mature forest, agricultural fields, and urban development. Both landscape composition and configuration can effect ecological processes and species' distributions (Freemark et al. 1995). Landscape composition includes the diversity and abundance of patch types, while configuration is the spatial location of patches within a landscape (Freemark et al. 1995). Composition and configuration can change temporally, either as a result of human activities or natural processes. Consequently, their effect on species' distributions likely is dynamic as well, but studies examining bird response to changing landscapes are lacking.

Most studies of the landscape ecology of birds were conducted in landscapes that have been permanently fragmented for years or even decades (Freemark et al. 1995). Forest size apparently has the greatest influence on species number in temperate landscapes. Factors such as patch isolation and vegetational composition could also be important, but less so than forest area. However, results of studies conducted in permanently fragmented landscapes may not be applicable to forested landscapes fragmented by timber harvests (McGarigal and McComb 1995). Few studies have been conducted in landscapes fragmented by silvicultural activities. Density of some songbird species are higher in fragmented areas than areas fragmented by timber harvesting in Maine (Hagan et al. 1996). Some species appear to move to the closest suitable habitat upon returning to their former breeding territories that have been harvested (Hagan et al. 1996). Thus, densities in the remaining habitat increase as birds crowd into the fragments of forest. In Missouri, some forest-interior species had lower abundance on the forests with clearcutting and some had higher abundance, while early successional species all had higher abundance (Thompson et al. 1992). Avian nest predators and cowbirds did not differ in abundance between forests with clearcuts and those without in Missouri, and forest-interior birds extensively used early and mid-successional even-aged stands (Thompson et al. 1992). Thus, fragmentation because of timber harvesting may be less severe than fragmentation because of land conversion. 
Hagan et al. (1996) and Thompson et al. (1992) worked in forests where the primary form of forest fragmentation was clearcutting. In other heavily forested regions of the East silvicultural alternatives to clearcutting, such as two-age harvesting, group selection, and singletree selection, are being employed. Less is known about these partial-harvesting techniques and how they impact birds returning to their breeding territories. Another common timber removal technique is diameter-limit, or sawtimber, harvesting where all trees greater than a predetermined diameter at breast height (dbh) are removed. Although this method is not based on a silvicultural system it is the most commonly used technique for removing trees in the eastern deciduous forest on private lands (Miller 1993). Despite the popularity of this method it has not been examined for its impacts on songbirds or other vertebrate species. Even less is known about how songbirds respond to different harvesting techniques from a landscape-level rather than a microhabitat-level perspective.

Many conservationists believe that public reserves do not constitute enough habitat to effectively conserve biodiversity and that private landowners need to be included in conservation efforts (Hansen et al. 1991). Harris (1984) suggests that public reserves be complemented by "seminatural" lands that are managed for commodity production as well as species diversity. In West Virginia, private landowners own approximately $90 \%$ of the state's forested lands (Birch et al. 1992). Rosenberg and Wells (1992) examined songbird population trends in the Northeast and determined that many species experiencing declines elsewhere were remaining stable or increasing in West Virginia. Clearly, studies determining how forest management practices on private lands impact songbird species in this state are needed before large-scale harvesting occurs.

The purpose of this study was to determine how forest management practices on an industrial forest in West Virginia immediately impact songbird abundance, nesting success, and habitat selection, and to determine if songbirds respond to different types of timber harvests as fragmentation events. Studies such as this provide valuable information which can be used to make predictions about which species are susceptible to declines because of timber management practices.

The specific objectives of this dissertation research were: 1) To assess songbird abundance and reproductive success on an industrial forest at the beginning of a 10-year cutting cycle; 2) To examine songbird abundance and reproductive success prior to and immediately 
following diameter-limit harvesting on a private industrial forest; 3) to examine songbird abundance and reproductive success prior to and immediately following two-age harvesting on a private industrial forest and; 4) to examine songbird habitat selection prior to and after timber harvesting at microhabitat- and landscape-levels. The objective of this chapter is to present inventory data of songbird abundance on an industrial forest, and to examine abundance among landtypes and among forest habitats over a 3-year period. Nest survival estimates in relation to forest habitats also are presented for this 3-year period.

\section{STUDY AREA}

The study area was the Westvaco Wildlife and Ecosystem Research Forest (WWERF) in southwestern Randolph County, West Virginia (384’N lat., $80^{\circ} 3^{\prime} \mathrm{W}$ long.)(Fig. 1.1). The 3360-ha forest was established by Westvaco in 1994 to study of the effects of industrial forestry management practices on Appalachian ecosystem components and processes. The forest is located on the unglaciated Allegheny Mountain and Plateau physiographic province with elevations ranging from 740 to $1200-\mathrm{m}$ (Fenneman 1938). The mountainous topography is characterized by broad plateau-like ridges, steep to moderate side slopes, and narrow valleys.

Most of the area is 60-80 year old secondary hardwood forests. These forests were established by natural regeneration following large-scale railroad harvesting in the early part of the 1900's (Clarkson 1993). Timber on the site has been thinned and harvested sporadically since the 1930's, and the forest is transected by numerous logging roads and skidder trails created by these activities. Currently, the WWERF is undergoing a 10-year harvest schedule throughout the site comprised of a mix of diameter-limit harvests for sawtimber production, and two-age and silvicultural clearcutting (seed tree harvests) for stand regeneration.

The climate is cool and moist, with average annual precipitation exceeding $155-\mathrm{cm}$ (Strausbaugh and Core 1977). Soils on the area are acidic and well-drained inceptisols (Stephenson 1993). Forest cover is primarily an Allegheny hardwood-northern hardwood type dominated by beech (Fagus grandifolia), yellow birch (Betula alleghaniensis), sugar maple (Acer saccharum) red maple (A. rubrum), black cherry (Prunus serotina), Fraser's magnolia (Magnolia fraseri), and red spruce (Picea rubens). Plant nomenclature follows Strausbaugh and Core (1977). At the lower elevations, species from the cove hardwood and mixed mesophytic associations, including tuliptree (Liriodendron tulipifera), basswood (Tilia americana), sweet 
birch (B. lenta), and northern red oak (Quercus rubra) occur. Riparian areas are characterized by hemlock (Tsuga canadensis)-red spruce-rhododendron (Rhododendron maximum) communities. Upland hardwoods dominated by chestnut oak (Q. prinus) and scarlet oak (Q. coccinea) occurred on some ridges and xeric slopes. On some exposed ridges, red spruce and eastern hemlock predominate. Throughout, rhododendron and striped maple (A. pensylvanicum) form dense understory thickets. As an artifact of past forest management activities and excessive deer herbivory, a dense coverage of hay-scented fern (Dennstaedtia punctilobula) occurs where the shrub layer is absent and the overstory canopy is not continuous.

Several timber harvests were conducted on the WWERF prior to the start of this study in May 1996. Diameter-limit harvesting occurred on 2 stands in 1994 and on 1 stand in early 1996. Two-age harvesting occurred on 2 stands, one in 1990 and one in 1994. One stand received a regeneration (seed tree) harvest in 1991, and another stand received a regeneration harvest immediately before the first field season (Fig. 1.2).

Between the 1996 and 1998 field seasons several stands had diameter-limit harvests, 2 stands had two-age harvests, and 2 stands had regeneration harvests (Fig. 1.2). In addition, several small blocks were harvested in 3 stands using a variety of harvesting methods (clearcut, two-age, shelterwood harvests) for a study examining impacts of forest management techniques on floral diversity and salamander abundance (Harpole and Haas 1999).

\section{METHODS}

\section{Songbird Abundance}

Songbird abundance was measured on the WWERF using standardized point count procedures (Ralph et al. 1993). One hundred-forty six point counts were conducted throughout the forest in 1996 and 1997 (Fig. 1. 3.). Two of these points were dropped in 1998 because of their close proximity to timber harvesting. Point counts were located on a 241-m x 241-m grid system previously established for forest inventory. Points were marked with an aluminum stake and uniquely numbered. A Trimble Global Positioning System ${ }^{\mathrm{TM}}$ (GPS) was used to geographically reference each point. Seventy-one points were placed 241-m apart (every grid point) within eight 39.7-ha nest searching plots. An additional 75 points were located throughout the forest and were separated by a distance of at least 482-m (every other grid point on every other line) in order to cover the entire area. The first line sampled was selected randomly as well 
as the first point on each line. Every other grid point on the line was sampled thereafter. However, due to logistical constraints, some points on the grid were excluded. Point count stations were found to be spatially independent using geostatistical analyses (Weakland and Wood, unpub. rept.).

I used 50-m fixed radius point counts to sample the songbird community. Each point count was surveyed twice between 20 May and 5 July each year of the study, once each by 2 different observers. The maximum number of birds detected between these 2 bouts was recorded as the index of abundance for each point count (Hagan et al. 1997). For example, if 3 ovenbirds (Seiurus aurocapillus) were recorded in the first bout, and 5 were recorded in the second bout, then 5 was used as the index.

Counts began at one-half hour after sunrise (approximately 0600 EST) and lasted until 4 hours after sunrise (approximately 1000 EST). All birds seen or heard singing/calling were recorded as either in or out of the 50-m radius circle. Birds were recorded to species as: male (singing), male (visual), female (visual), male (flyover), female (flyover), unknown sex (calling), unknown sex (visual), unknown sex (flyover). Birds recorded as flyovers were not used in the index of abundance. If a song or call was not identified to species in the field, an attempt to determine the species was made upon returning to the office. If the observer could not identify the call or song the species was listed as unknown. Counts lasted 10-min and began approximately 1 minute after the observer reached the point count station. Birds were recorded in 3 time blocks: 0-3-min, >3-5-min, and >5-10-min to allow comparison with other studies. Counts were not conducted when it was raining or when noise from wind interfered with counting. Weather conditions and wind speed were recorded using the indices found in Table 1.1 (Martin et al. 1997).

Observers were trained for a period of 2-3 days before starting actual surveys. Two observers conducted counts at the same station and compared species observed and distances estimated. They also practiced distance estimation by pacing 50-m from the point count center after the practice session and by pacing to the approximate location of a singing bird. At least 10 practice sessions were conducted before beginning actual surveys.

Songbird species were placed into nesting guilds and habitat groups based on Freemark and Collins (1992) and Ehrlich et al. (1988) and on my own experience of the species nesting on the WWERF. Nesting guilds included: ground-, shrub-, subcanopy-, canopy-, and cavity- 
nesters. Habitat groups included: forest-interior, interior-edge, and edge species. Table 1.2. lists all species observed on the WWERF, their scientific names, and their habitat and nesting guild classifications. Estimated means, standard errors, and 95\% confidence intervals of abundance and frequency of occurrence are reported for all species encountered on the WWERF and for each guild/group by year, landtype, and forest habitat. Species nomenclature follows the American Ornithologists' Union checklist of North American birds (American Ornithologists' Union 1999). Abundance estimates were log-transformed before analyses to help normalize residuals (Nur et al. 1999). Neotropical migrant birds species listed as "conservation priorities" by the West Virginia Partners in Flight(WV PIF) research working group (West Virginia Partners in Flight 1999) also are discussed relevant to their occurrence on the WWERF.

Landtype classes and forest habitats were based on Westvaco inventory data, but were modified for this study. A landtype classification with 14 categories was developed for the WWERF (R. Odum, pers. comm.). I combined these categories into 4 landtypes: submesic slope, mesic slope, xeric slope, and ridge (Table 1.3). Only 3 point counts were located along stream terraces; these were included in the submesic slope class. Only points that were not harvested during the 3 years of the study were used in the analysis of landtype effects. I compared species abundance among the 4 landtypes using two-way analysis of variance (ANOVA), with year and landtype as classes. I tested for landtype effects and for year by landtype interactions. If interactions were detected, subsequent ANOVA's were performed on each year separately to determine the effects of the interactions. If a landtype effect was detected, a subsequent Scheffe's multiple comparison test was conducted to determine differences between the least squared means of each pair of landtypes (Neter et al. 1996).

Forest habitats were based on Westvaco inventory data and were defined as the following: mature hardwoods, mature hardwoods/softwoods, regeneration ( $\leq 3$ year old seed tree and two-age harvests), diameter-limit harvests, and open/non-forested areas. Mature hardwoods were a combination of northern hardwoods and upland hardwoods because too few points were in upland hardwoods to analyze separately. Mature hardwoods/softwoods (mixed woods) were a mix of hardwoods, eastern hemlock, and/or red spruce. These included stands that had been classified by Westvaco as either cove forests, upland hardwoods, or northern hardwoods, but I chose to separate them out because of the high amount of conifer cover in the stand based on aerial photographs and ground truthing. Seed tree and two-age regeneration harvests were 
combined because they had similar structure, canopy cover, and residual basal area $\left(\sim 9.5-\mathrm{m}^{2} / \mathrm{ha}\right)$ after harvesting (pers. obs.). Open/nonforested areas included roads, log landings, and mineral (rock) extraction areas, but no point counts were located in these areas. Too few points were located in regeneration stands that were $>3-20$ years old to include in analyses. Thus, the analysis of forest habitats included mature hardwoods and mature mixed woods, diameter-limit harvests (basal area 20- $\mathrm{m}^{2} / \mathrm{ha}$ ), and two-age harvests in 1997-98. Diameter-limit harvests had approximately 500 residual trees/ha (>8-cm dbh), and two-age harvests had approximately 169 residual trees/ha. Unharvested stands had >520 trees/ha.

Analysis of covariance (ANCOVA) was used to examine songbird abundances among forest habitats on the WWERF in 1997-1998 (Neter et al. 1996). Pre-harvest abundance (1996) was used as a covariate in this model. An important assumption in ANCOVA is that the slope of the regression line of the covariate by independent variable must be the same for all factor levels of the independent variable (Cody and Smith 1991). I tested for homogeneity of slopes before running the ANCOVA for each species and guild. If a species or guild showed a significant preharvest abundance by habitat interaction, then I did not use ANCOVA (Cody and Smith 1991). For these species I used ANOVA to examine post-harvest abundance among forest habitats (Neter et al. 1996). If a significant difference was found among habitat, I used the Sheffe' multiple comparison test to examine differences between habitat least-squared means. The family significance level for these tests was 0.10 (Neter et al. 1996).

A vertical structure index (VSI) also was used as a covariate in some models because detectability of songbirds may vary among habitat types (Pendelton 1995). The index was calculated following Nichols (1996) (Table 1.4). The percent canopy cover in 6 layer classes (Table 1.4) was determined using the ocular site-tube method of James and Shugart (1970). Four 0.04-ha subplots were established within the 50-m radius point count circle. One subplot was centered on the grid stake, and the other 3 were located $35-\mathrm{m}$ away at $0^{\circ}, 120^{\circ}$, and $240^{\circ}$. Twenty presence/absence site-tube readings were taken every $2.26-\mathrm{m}$ along two $22.6-\mathrm{m}$ perpendicular transects bisecting the center of each subplot, once for each canopy layer . Transects were established with 1 oriented perpendicular to the slope and the other parallel to the slope (Martin et al. 1997). The average canopy cover in each layer class from the 4 subplots was used to calculate the vertical structure index. This index was included in the model if it was 
correlated to a species abundance and if the test for lack of homogeneity of slopes was not significant.

Proc GLM (SAS ${ }^{\circledR}$ Institute, 1991) was used to conduct all ANOVA and ANCOVA tests. Proc CORR (SAS ${ }^{\circledR}$ Institute 1991) was used to examine correlations between pre- and postharvest abundance and between abundance and VSI. Tests were considered significant at the $\mathrm{P} \leq$ 0.05 level. Error rates were controlled for all treatment comparisons for a bird species, but not experiment-wide for all species.

\section{Nest Searching}

To examine effects of harvesting on avian reproductive success, I established 8 nest searching plots on the forest. Two plots were established in areas scheduled to receive diameterlimit harvests; 2 were established in areas scheduled to receive two-age harvests; and 4 plots were established as controls. Plots were paired spatially with a harvested and a coch Forest, Randolph County, West Virginiaize spatial variability. Approximately $1 / 2$ of each diameter-limit plot was harvested and approximately $1 / 3$ of each two-age plot was harvested in 1997 . Nests were placed into 6 treatments after harvesting: diameter-limit, diameter-limit periphery, diameter-limit control, two-age, two-age periphery, and two-age control. The plots were established around the existing grid system in a block pattern consisting of 9 ( $3 \times 3)$ point count stations (Fig. 1.3). The plots extended $90-\mathrm{m}$ past the corner stations and were 40 -ha in area. A new 60-m x 60-m grid system was established on the point count grid on each plot using vinyl flagging as an aid in relocating nests.

Four full-time nest searchers were employed to searched for and monitored nests each year. Each nest searcher was assigned 2 plots, and they visited each plot every 2-3 days (Martin et al. 1997). Nest searching methods followed Martin and Guepel (1993) and Martin et al. (1997). Nests were located by observing parental behavior, by systematically searching vegetation, and by flushing birds off nests. The method for finding each nest was recorded in order to determine search efficiency (Martin et al. 1997). Nests were monitored every 3-4 days to determine activity.

Contents were checked when the nest was initially located and on the next following visit. After the second visit nests were only approached and contents checked when it was estimated that nests were close to transition periods from egg-laying to incubation, from incubation to brooding, or from brooding to fledging. Searchers observed nests from a distance 
to determine if the nest was still active between transition dates. An attempt was made to estimate the transition dates (onset of incubation, hatching, and fledging) for each nest based on the species' nest cycle information found in the Breeding Bird Research Database (BBIRD) (Martin et al. 1997). Attempts were made to locate fledglings or to observe parent-fledgling interactions for nests found empty near the fledging date. If neither fledglings or interactions were observed, a nest was considered to have fledged if it was found empty within 2 days of estimated fledge date (Martin et al. 1997). A nest also was considered to have successfully fledged young if nestlings fledged as a result of the observer checking the nest. Searchers avoided leaving dead-end scent trails to nests and did not approach nests when avian predators were observed in the vicinity of the nest in order to minimize predation (Martin et al. 1997). Vinyl flagging was placed at least 15-m away from the nest if required for relocation.

Nest survival was calculated using the Mayfield method (Mayfield 1961, Mayfield 1975, Johnson 1979) for all species for which nests were located. Daily survival estimates were calculated for the incubation and brood periods separately because there may be differential survival between these two periods. Daily survival estimates were calculated using the typical incubation days and brood days reported either in Ehrlich et al. (1988) or the BBIRD database for each species (Martin et al. 1997). If no values were available for a particular species in either source, then an estimate of the typical days for each period were made based on the average incubation and brood days of nests that were followed for a complete nest cycle on the WWERF. Daily survival (DS) estimates for each period for each species were calculated by:

$$
\mathrm{DS}_{\text {period }}=(1-\text { mortality })
$$

Where

period $=$ incubation or brood

mortality $=$ number nests failed/exposure days

Survival (S) for each period was calculated as

$$
\mathrm{S}_{\text {period }}=\text { daily survival }{ }^{\text {nest days }}
$$

Where

nest days $=$ typical number of days in that period for that species.

Total survival (TS) for the whole nest cycle was the product of $S_{\text {incubation }}$ and $S_{\text {brood }}$. 
Nest survival estimates also were obtained for all nests combined and for the nesting guilds. Nest days for incubation and brood periods for each of these guild was determined by taking the average of number of days for each species in each guild on each nest plot. Estimates of survival for all species combined were determined in the same manner. Survival estimates are reported in this chapter by year and treatment. It has been suggested that at least 20 nests of each species should be found in each treatment each year in order to conduct statistical analysis (Martin et al. 1997). In this study <20 nests were found for each species, and, thus, I only report survival estimates.

Habitat surrounding each nest found was measured following BBIRD protocals (Martin et al. 1997). A 0.04-ha circular plot was established with the nest at the center. All trees $>8-\mathrm{cm}$ dbh within the plot were counted and placed into two size classes: $>8-29.9-\mathrm{cm}$ and $\geq 30-\mathrm{cm}$. In a 0.008-ha plot centered on the nest, all trees $>0.5-\mathrm{m}$ tall and $\geq 2.5-\mathrm{cm}-8 \mathrm{~cm}$ dbh were counted. Additionally, all snags $>1.4-\mathrm{m}$ high and $\geq 12.0$-cm dbh were counted within the 0.04-ha plot. Percent canopy cover and percent ground cover estimates were taken in the same manner as on point count plots (James and Shugart 1970). Ground cover categories were green vegetation, down wood, bareground/rock, and litter. Green vegetation included grasses, ferns, herbaceous plants, moss, and trees $<0.5$-m high. Down wood was any $\log \geq 4.0-\mathrm{cm}$ in diameter. Distance to nearest edge, estimated by pacing, also was included as a variable. Tree and understory stem densities were transformed using the equation $\mathrm{Z}=\log _{10}($ variable +1$)$, and percentage data were subjected to an arcsine-square root transformation (Dowdy and Wearden 1991).

I used logistic regression (Neter et al. 1996) to determine which of these habitat variables were significant predictors of nest survival or predation. The significance level chosen for entry and retention in the model was 0.10 . The likelihood ratio chi-square test was used to determine the significance of the addition of each variable to the logistic regression model (Stokes et al. 1995). Variables that failed the likelihood ratio chi-square test $(\mathrm{P} \leq 0.10)$ were not included in the model. I used the Hosmer-Lemeshow goodness-of-fit test to examine the performance of the model (Stokes et al. 1995). The SAS ${ }^{\circledR}$ logistic procedure was used to conduct this analysis $\left(\right.$ SAS $^{\circledR}$ Institute 1991). 


\section{RESULTS}

\section{Songbird Abundance}

\section{Years}

A total of 5,374 birds of 59 species were counted within point count plots on the WWERF from 1996-1998. Crows, jays, and woodpeckers were counted, and raptor and game species were noted but were not included in analyses (Table 1.5). Abundance was calculated for the 59 songbird species detected (Table 1.6). The 5 most abundant (Table 1.6) and most frequently occurring species (Table 1.7) were: the red-eyed vireo (Vireo olivaceus), blue-headed vireo (Vireo solitarius), black-throated green warbler (Dendroica virens), Canada warbler (Wilsonia canadensis), and veery (Catharus fuscescens). The habitat group with the highest abundance was the forest-interior group which occurred at almost all point counts each year (Table 1.7). Ground, subcanopy, and canopy nesters were the most abundant nesting guilds (Tables 1.6-1.7). Sixteen species were recorded at $\geq 20 \%$ of point counts each year (Table 1.7).

The abundance of several species (Tabe 1.8) and guilds differed among years (Fig. 1.4). Total bird abundance and abundance of forest-interior, edge, and canopy-nesting species decreased 1- and 2-years after harvesting (Fig. 1.4). Abundance of shrub nesters initially decreased 1-year after harvesting, then increased in 1998 to pre-harvest levels. Interior-edge species abundance was similar in 1996 and 1998. Subcanopy nesters significantly decreased in abundance 1 year after harvesting, then increased in abundance to greater than 1996 pre-harvest levels. Species had varying abundances among years, with some increasing after harvesting and others decreasing (Table 1.8).

\section{Landtypes}

Mean abundance for each species and guild by landtype are given in Table 1.9. The ground nesting guild had higher abundance on xeric slopes than on mesic slopes (Table 1.10). Abundance of canopy nesters was lower on xeric slopes than on the other landtypes (Table 1.10). Total abundance and abundance of habitat groups did not vary by landtype. Shrub, subcanopy, and cavity nesters also did not vary by landtype (Table 1.10).

Black-throated green warblers had lower abundance on xeric slopes than on submesic slopes and on ridge tops. The blue-headed vireo had higher abundance on xeric slopes than ridges, while the both the Canada warbler and the winter wren (Troglodytes troglodytes) were less abundant on ridges than on the other 3 landtypes. The dark-eyed junco (Junco hyemalis) 
showed a preference for submesic slopes over xeric slopes and ridges. The ovenbird was found at higher abundances on ridges than in other landtypes, whereas the eastern towhee (Pipilo erythrophthalmus) had higher abundance on xeric slopes than on any other landtype. The veery had higher abundance on xeric slopes than on ridges and mesic slopes, and it had higher abundance on submesic slopes than mesic slopes. There was a significant year by landtype interaction $(\mathrm{F}=3.66, \mathrm{P}<0.01)$ for the rose-breasted grosbeak (Pheuticus ludovicianus). This species had higher abundance on ridges than on submesic slopes in 1996, but did not show differences among landtypes in 1997 and 1998 (Table 1.10).

\section{Forest Habitats}

Post-harvest abundance was correlated to pre-harvest abundance for most species. Thus, pre-harvest abundance was included as a covariate in the models examining the influence of forest habitat on abundance after harvesting. Four species, the veery, winter wren, Canada warbler, and dark-eyed junco failed the test for homogeneity of slopes among habitats (i.e. they had significant pre-harvest abundance by habitat interactions). Thus, pre-harvest abundance was not included as a covariate in models for these species. Several species and guilds also were correlated to the VSI. VSI was included in models for those species and guilds where there was not a significant VSI by habitat interaction. Means and standard errors for each guild and species by habitat type and year are given in Table 1.11 .

Total songbird abundance was significantly higher in diameter-limit and two-age harvests than in mature hardwoods or mixed woods (Table 1.11). Interior-edge species had higher abundance in two-age harvests than any other habitat, and they also were more abundant in diameter-limit harvests than in mature forests (Table 1.12). There was a significant year by habitat interaction for the edge group $(\mathrm{F}=3.72, \mathrm{P}=0.02)$. However, in both 1997 and 1998, edge species were more abundant in two-age harvests than in any other habitat. Ground nesters were more abundant in diameter-limit harvests and in two-age harvests than either mature habitat. Shrub nesters were more abundant in two-age harvests than any other habitat and more abundant in mature hardwoods than mixed woods. Cavity nesters were more abundant in the two-age and diameter-limit harvests than in either mature habitat (Table 1.12).

Species varied in their habitat use. Black-throated blue warblers (Dendroica caerulescens), blue jays (Cyanocitta cristata), red-eyed vireos, and veerys did not differ in abundance among habitat type (Table 1.12). Black-capped chickadees (Poecile atricapillus), 
dark-eyed juncos, and rose-breasted grosbeaks, all interior-edge species, had higher abundance in diameter-limit harvests than mature habitats. The scarlet tanager (Piranga olivacea) had higher abundance in two-age harvests than any other habitat type (Table 1.12). The American crow (Corvus brachyrhynchos), an edge species, also had higher abundance in two-age harvests than in mature mixed woods and diameter-limit harvests. The black-throated green warbler had lower abundance in two-age harvests than in diameter-limits and mature hardwoods. Ovenbirds appeared to prefer mature hardwoods over any other habitat type, and the eastern towhee preferred two-age harvests over other habitats. Winter wrens had higher abundance in mature mixed woods and diameter-limits than mature hardwoods. Blue-headed vireos were higher in diameter-limit harvests than in mature hardwoods (Table 1.12). The Canada warbler had higher abundance in mature mixed woods than in mature hardwoods. Magnolia warblers (Dendroica magnolia) showed a significant year by habitat interaction $(\mathrm{F}=3.73, \mathrm{P}=0.01)$. In 1997 they were more abundant in diameter-limit harvests than any other habitat. They were more abundant in mature mixed woods than mature hardwoods and two-age harvests and more abundant in diameter-limit harvests than mature hardwoods in 1998 (Table 1.12).

\section{Partners in Flight Conservation Priority Species}

Twelve species observed on the WWERF are listed as "conservation priority" species by WV PIF. These species were: black-billed cuckoo (Coccyzus erythropthalmus), worm-eating warbler (Helmitheros vermivora), red-eyed vireo, cerulean warbler (Dendroica cerulea), hooded warbler (Wilsonia citrina), yellow-billed cuckoo (Coccyzus americanus), eastern wood-peewee (Contopus virens), acadian flycatcher (Empidonax virescens), Louisiana waterthrush (Seirus motacilla), scarlet tanager, yellow-throated vireo (V. flavifrons), and wood thrush (Hylocichla mustelina). Only 2 of these species, the red-eyed vireo and scarlet tanager, were observed at $\geq 20 \%$ of point counts on the WWERF. The other species were encountered much less frequently (Table 1.6). The black-billed cuckoo, worm-eating warbler, cerulean warbler, yellow-billed cuckoo, eastern wood-peewee, Louisiana waterthrush, and yellow-throated vireo were found from $<1.0 \%$ to $6.0 \%$ of point counts from 1996-1998. The hooded warbler, acadian flycatcher, and wood thrush were found more frequently (Table 1.6). The hooded warbler was most often observed at lower elevations in stands with a dense understory of greenbrier. Acadian flycatchers were restricted to riparian areas, and.the wood thrush was observed most frequently at lower elevations at points with well-developed understories. 


\section{Nest Survival}

One-hundred ninety-nine nests were found from 1996-1998 on the WWERF. Survival for all 3 years combined was $43.3 \%$ during incubation and $61.3 \%$ during brooding. Total survival for all 3 years was $25.6 \%$. The highest survival for any nesting guild was $35.3 \%$ for cavity nesters $(n=5)$, followed by canopy nesters $(33.1 \%, n=22)$, ground nesters $(27.3 \%, n=100)$, shrub nesters $(23.5 \%, \mathrm{n}=27)$, and subcanopy nesters $(21.3 \%, \mathrm{n}=45)$. Survival estimates by year were $19.2 \%, 23.5 \%$, and $35.2 \%$ in 1996, 1997, and 1998, respectively. Table 1.13 lists the number of nests found per treatment per year as well as exposure days and daily survival estimates for each nesting period. Predation was the primary cause of nest failure. Ninety-two percent of all nests known to have failed were lost to predators. The percent of nest failures because of predation by year was: $89 \%$ in 1996, $94 \%$ in 1997, and $93 \%$ in 1998. Only one nest, a veery in 1997, was parasitized by brown-headed cowbirds $(0.5 \%)$. Lists of nests found by species in each treatment and nesting guild are found in Chapters 2 and 3 of this volume. Habitat was similar between successful and predated nests (Table 1.14).

\section{DISCUSSION}

My results indicate that abundance for many songbird populations on the WWERF varied by year. Several local factors may have influenced these results. First, there were several timber harvests each year, reducing the amount of forest canopy cover on the forest. Most species recorded were forest-interior species or canopy-nesting species. It is not surprising that both of these groups had lower abundance in 1997 and 1998 following harvest than in 1996. The blueheaded vireo and the veery, both interior species, also had lower abundance in 1997-98 than in 1996. Interior-edge species and subcanopy-nesting species, on the other hand, appeared to increase after 1996, likely as a result of increased edge habitat from timber harvesting. However, these results may largely be due to increases in the red-eyed vireo in 1998, which was the most commonly occurring species on the WWERF. Neither edge species nor shrub-nesting species increased after harvesting. However, as harvested stands begin to regenerate it is likely that both of these groups will increase on the forest.

One factor influencing abundance of ground and shrub nesters on the forest is the impact of white-tailed deer herbivory on understory stem density. High densities of white-tailed deer reduced the number of shrub-nesting birds (deCalesta 1994) and decreased bird species richness 
and diversity in Pennsylvania managed forests (Stout et al. 1992). High deer densities also result in reduced species richness and abundance of herbaceous and woody vegetation (Alverson et al. 1988, Tilghman 1989). Songbird richness and abundance have been correlated to tree species abundance and composition as well as to vegetation structure (MacArthur and MacArthur 1961, DeGraaf et al. 1991). Much of the understory on the WWERF has been depleted because of high deer densities (B. Collins, pers. comm., K. Miller, pers. comm.). Shrub nesters in particular had lower abundance on the forest than other species and were often restricted to areas with high densities of rhododendron or greenbrier (pers. obs.).

Ground-nesting species such as the eastern towhee are known to respond to increases in understory vegetation (Bell and Whitmore 1997). Several ground-nesting species were positively correlated to the VSI, indicating a preference for areas with several layers of canopy. Nests on the ground also may be trampled by deer; one dark-eyed junco nest was found destroyed with deer tracks in close proximity. I also observed a significant amount of browsed vegetation on the WWERF as well as an abundance of deer pellet groups (pers. obs).

Results indicate that some species and guilds show a preference among landtypes. Shrub nesters had higher abundance on xeric slopes than on mesic slopes, perhaps because of high greenbrier abundance, which is used as a nesting substrate in these areas. Canopy-nesting species also had lower abundance on xeric slopes than on the other landtypes. Lower songbird densities have been reported elsewhere in xeric habitats (Shugart and James 1973, Smith 1977). In the Ozark region, lower arthropod abundance in xeric habitats contributed to low songbird densities (Smith 1977).

Abundance of 4 ground nesters, the eastern towhee, ovenbird, Canada warbler, and winter wren differed among landtypes. The eastern towhee preferred xeric slopes over other landtypes, likely because of the presence of greenbrier in these areas. The Canada warbler and winter wren were less abundant on ridges, suggesting that these species prefer moist habitats. The ovenbird was more abundant on ridges in this study, similar to findings in the Ozarks (Smith 1977).

There were significant differences in songbird abundance among the forest habitat types. Most interior-edge species appeared to prefer two-age and diameter-limit harvests, and none had lower abundance in harvests than in mature forests. Black-capped chickadees, dark-eyed juncos, and rose-breasted grosbeaks were more abundant in diameter-limit harvests than in mature forest 
habitats, while the eastern towhee was more abundant in two-age harvests than in any other habitat.

The black-throated green warbler was less abundant in two-age harvests than mature hardwoods and diameter-limit harvests. This species might have been avoiding the two-age harvests because of the reduced canopy cover caused by the harvesting. Thompson and Capen (1988) examined songbird populations in several deciduous and deciduous-coniferous sawtimber stages created by silvicultural activities in a Vermont forest. They placed the black-throated green warbler and the blue-headed vireo in closed-canopy mixed deciduous-coniferous forests. On the WWERF, these 2 species were not more abundant in mixed woods than in hardwoods, and blue-headed vireo abundance did not differ between mixed woods and two-age harvests. Both of these species may have a wider range of tolerance in this industrial forest landscape than in Vermont. Surprisingly, 1 forest-interior, canopy-nesting species, the scarlet tanager, was found in higher abundance in two-age harvests. However, this species is known to tolerate a wide variety of forested habitats, including clearcuts (Crawford et al. 1981,Yahner 1993). The ovenbird was more abundant in mature hardwoods than in any other habitat, a result that concurred with Thompson and Capen (1988). The magnolia warbler appeared to prefer mature mixed woods and diameter-limit harvests to two-age harvests; this result also was similar to Thompson and Capen (1988) who placed this species in an open-canopy association.

Nest survival results are inconclusive because of the low number of nests that were found. However, overall nest survival estimates on the WWERF (25.6\%) appear to be lower than in other areas of West Virginia. Wood et al. (1998) found survival estimates ranging from $31 \%$ to $49 \%$ in unharvested stands, clearcut stands, and two-age stands on the Monongahela National Forest (MNF). Demeo (1999), who examined songbird abundances and nest survival at multiple scales on the MNF during the same time period as my study, found nest survival estimates ranging from $23 \%$ to $39 \%$ (average=30\%). Nest survival may be lower on the WWERF for several reasons. First, the WWERF has a large number of logging trails and roads throughout the forest that create hard edge habitat and travel lanes for predators. Second, deer browsing has impacted the understory and has likely reduced the amount of vertical structure, and, consequently, nesting cover, for songbirds. Lower amounts of nesting cover may lead to increases in predation from predators such as blue jays, American crows, common ravens (Corvus corax), eastern chipmunks (Tamias striatus), white-footed mice (Peromyscus 
leucopus), striped skunks (Mephistis mephistis), red squirrels (Tamiasciurus hudsonicus), southern flying squirrels (Glaucomys volans), black bears (Ursus americanus), and raccoons (Procyon lotor). Lastly, reduced vertical structure reduces the number of niches available for insects and other invertebrates and therefore decreases the abundance of insects upon which many species of songbirds depend. Duguay et al. (in press) found higher nest survival estimates in areas with higher insect abundance on the MNF. Higher insect abundance may increase nest survival because parent birds can spend less time foraging and more time defending the nest from predators.

In conclusion, partial timber harvesting practices on the WWERF do not appear to have significant immediate impacts on the abundance of most common songbird species. However, long-term studies are needed to determine if songbird populations will continue to remain stable on the WWERF. In addition, it would be helpful to examine songbird populations simultaneously on several industrial forests, non-industrial forests, and on non-working forests in different regions to determine both localized and regional effects of timber harvesting and fragmentation on songbird abundances. I also suggest that insect abundance be investigated as a potential factor influencing abundances and nest survival in an industrial forestry landscape. Also, very little information is available concerning avian dispersal and survival rates. Colorbanding studies should be conducted to determine if differences in adult and juvenile survival rates exist between industrial and non-industrial forests.

\section{MANAGEMENT IMPLICATIONS}

I recommend that deer density be reduced on the WWERF to increase understory vegetation and to provide increased nesting habitat for ground-, shrub-, and subcanopy-nesting species (deCalesta 1994). A reduction in deer density also will allow increased regeneration of hardwood forests (Tilghman 1989). For forests under intense management such as the WWERF, white-tailed deer densities should approximate $8 \mathrm{deer} / \mathrm{km}^{2}$ to facilitate hardwood regeneration (Behrend et al. 1970, Tilghman 1989). At this deer density, songbird populations and richness will likely be maintained (deCalesta 1994).

Timber harvesting does not appear to have immediate negative impacts on most common species of songbirds on the WWERF. Forest-interior songbird populations, however, may decrease if the amount of regeneration harvests, that are not regenerating because of deer 
herbivory, increases on the forest. Management for songbird diversity on the WWERF could be approached several ways. First, forest managers could plan single-tree selection harvests to maintain canopy closure and increase vertical diversity for forest-interior species and species that use canopy gaps (Thompson et al. 1995). Several species on the WV PIF conservation priority list, such as the hooded warbler, red-eyed vireo, scarlet tanager, and yellow-throated vireo, would benefit from these harvests. In addition to selection harvests, managers also could harvest some stands using seed tree and two-age harvests to increase habitat for early successional species that prefer large blocks of habitat (Thompson et al 1995). Early successional species that prefer larger blocks include blue-winged warblers (Vermivora pinus), golden-winged warblers (V. chrysoptera), and prairie warblers (Dendroica discolor), which are all on the WV PIF conservation priority list. Additionally, regeneration harvests could be "clumped" to decrease isolation effects and potentially to reduce deer herbivory (Thompson et al. 1995). Interior-edge species also would benefit from selection harvests and regeneration harvests.

Another approach would be to increase the amount of regeneration harvests on the forest and increase rotation length to $>120$ years. In this approach, edge and early successional species would benefit from the increase in the amount of young and regenerating forest, which is a habitat that is scarce in West Virginia (Bell and Whitmore 1997). Forest-interior species would benefit from the longer rotation lengths. In both approaches, age classes should be balanced across the forest because each stage of succession is important for some species (Thompson et al. 1995).

\section{ACKOWLEDGEMENTS}

This research was funded by Westvaco Corporation, Forest Resources Division, and the West Virginia Division of Natural Resources Nongame Wildlife and Natural Heritage Program. The West Virginia Cooperative Fish and Wildlife Research Unit, USGS, BRD, provided equipment and logistical support. I would like to thank Sydney McIntyre for his assistance in the field, and Dr. Richard Odum, Sally Lane and Sarah Clapham for their assistance with maps and GIS data. I would especially like to thank W. Mark Ford for his input into this project and for his efforts to provide me with up-to-date information about activities on the WWERF. I also thank the many field technicians who assisted with nest searching, point count surveys, and 
habitat sampling. L. S. Gribko, A. F. Egan, W. M. Ford, and R. C. Whitmore provided helpful suggestions on earlier drafts of this manuscript.

\section{LITERATURE CITED}

ALVERSON, W. W., D. M. WALLER, AND S. L. SOLHEIM. 1988. Forests too deer: edge effects in northern Wisconsin. Conservation Biology 2:348-358.

AMERICAN ORNITHOLOGISTS' UNION. 1999. The American Ornithologists' Union checklist of North American birds. http://pica.wru.umt.edu/AOU/birdlist.HTML.

BEHREND, D. F., G. F. MATTFIELD, W. C. TIERSON, AND J. E. WILEY III. 1970. Deer density control for comprehensive forest management. Journal of Forestry 68: 695-700.

BELL, J.L., AND R.C. WHITMORE. 1997. Eastern towhee numbers increase following defoliation by gypsy moths. Auk 114: 708-716.

BIRCH, T.W., D.A. GASNER., S.L. ARNER, AND R.H. WIDMANN. 1992. Cutting activity on West Virginia timberlands. Northern Journal of Applied Forestry 9: 146-148.

BRITTINGHAM, M., AND S. TEMPLE. 1983. Have cowbirds caused forest songbirds to decline? Bioscience 33:31-35.

CLARKSON, R.B. 1993. Destruction of the upland forest by lumbering and fire. Pages 35-46 in S.L. Stephenson, ed.,Upland forests of West Virginia. McCain Printing Co., Parsons, West Virginia.

CODY, R.P., AND J.K. SMITH. 1997. Applied statistics and the SAS programming language. Prentice Hall, Upper Saddle River, New Jersey. 445pp.

CRAWFORD, H.S., R.G. HOOPER, AND R.W. TITTERINGTON. 1981. Songbird population rsponse to silvicultural practices in central Appalachian hardwoods. Journal of Wildlife Management 45: 680-692.

DECALESTA, D.S. 1994. The effects of white-tailed deer on songbirds within managed forests in Pennsylvania. Journal of Wildlife Management 58:711-718.

DEGRAAF, R. M., V. E. SCOTT, R. H. HAMRE, L. ERNST, AND S. H. ANDERSON. 1991. Forest and rangeland birds of the United States. U. S. Dept. Agric. Handb. 688. 625pp.

DEMEO, T. E. 1999. Forest songbird abundance and viability at multiple scales on the Monongahela National Forest, West Virginia. Ph.D. dissertation, West Virginia University, Morgantown, West Virginia. 159pp. 
DOWDY, S. AND S. WEARDEN. 1991. Statistics for research. John Wiley and Sons. New York, New York, 629pp.

DUGUAY, J.P., P.B. WOOD, AND G.W. MILLER. In press. Effects of timber harvests on invertebrate biomass and avian nest success. Wildlife Society Bulletin.

DUNNING, J. B., B. J. DANIELSON, AND H. R. PULLIAM. 1992. Ecological processes that affect populations in complex landscapes. Oikos 65:169-175.

EHRLICH, P.R., D.S. DOBKIN, AND D. WHEYE. 1988. The Birder's Handbook. Simon and Schuster Inc. New York, New York. 785pp.

FAABORG, J., M. BRITTINGHAM, T. DONOVAN, AND J. BLAKE. 1995. Habitat fragmentation in the temperate zone. Pages 357-380 in T.E. Martin and D. M. Finch eds., Ecology and management of neotropical migratory birds. Oxford University Press, New York and Oxford.

FENNEMAN, N.M. 1938. Physiography of the Eastern United States. McGraw-Hill, New York, New York, 714pp.

FINCH, D. M. 1991. Population ecology, habitat requirements, and conservation of neotropical migratory birds. U.S. For. Serv. Gen. Tech. Rep. RM-205.

FREEMARK, K. AND B. COLLINS. 1992. Landscape ecology of breeding birds in temperate forest fragments. Pages 443-454 in J.M. Hagan, III and D.W. Johnston, eds., Ecology and Conservation of Neotropical Migrant Landbirds. Smithsonian Institution Press, Washington and London.

FREEMARK, K., J. B. DUNNING, S. J. HEIJL, AND J. R. PROBST. 1995. A landscape ecology perspective for research, conservation, and management. Pages 381-427 in T.E. Martin and D. M. Finch, eds., Ecology and management of neotropical migratory birds. Oxford University Press, New York and Oxford.

GATES, J. E., AND L.W. GYSEL. 1978. Avian nest dispersion and fledgling success in fieldforest ecotones. Ecology 58:871-883.

HAGAN, J.M., and JOHNSTON, D.W. (Eds.). 1992. Ecology and Conservation of Neotropical Migrant Landbirds. Smithsonian Institution Press, Washington and London.

HAGAN, J.M., III, P.S. MCKINLEY, A.L. MEEHAN, AND A.L. GROVE. 1997. Diversity and abundance of landbirds in a northeastern industrial forest. Journal of Wildlife Management 61: 718-735. 
HAGAN, J.M., W.M. VANDER HAEGEN, and P.S. MCKINLEY. 1996. The early development of forest fragmentation effects on birds. Conservation Biology 10:188-202.

HANSEN, A. J., T. A. SPIES, F. J. SWANSON, AND J. L. OHMANN. 1991. Conserving biodiversity in managed forests. Bioscience 41:382-392.

HARPOLE, D. N. AND C. A. HAAS. 1999. Effects of seven silvicultural treatments on terrestrial salamanders. Forest Ecology and Management 114:349-56.

HARRIS, L.D. 1984. The fragmented forest. Univ. of Chicago Press, Chicago, Illinois.

HARTLEY, M., AND M.L. HUNTER, Jr. 1998. A meta-analysis of forest cover, edge effects, and artificial nest predation rates. Conservation Biology 12:465-469.

JAMES, F. C., AND C. E. MCCULLOCH. 1995. The strength of inferences about causes of trends in populations. Pages 41-54 in T.E. Martin and D. M. Finch, eds., Ecology and management of neotropical migratory birds. Oxford University Press, New York and Oxford.

JAMES, F.C., AND H.H. SHUGART. 1970. A quantitative method of habitat description. Audubon Field Notes 24: 727-736.

LORD, J. M., AND D. A. NORTON. 1990. Scale and the spatial concept of fragmentation. Conservation Biology 4:197-202.

MACARTHUR, R. H., AND J. W. MACARTHUR. 1961. On bird species diversity. Ecology 50:594-598.

MARTIN, T.E., AND G.R. GUEPEL. 1993. Nest-monitoring plots: methods for locating and monitoring success. Journal of Field. Ornithology 64: 507-519.

MARTIN, T.E., C. PAINE, C.J. CONWAY, W. M. HOCHACHKA, P. ALLEN, and W. JENKINS. 1997. BBIRD Field Protocol. USGS, Biological Resources Division, Montana Cooperative Fish and Wildlife Research Unit, Missoula, Montana.

MAYFIELD, H.F. 1961. Nesting success calculated from exposure. Wilson Bulletin 73: 255261.

MAYFIELD, H.F. 1975. Suggestions for calculating nest success. Wilson Bulletin 87: 456466.

MCGARIGAL, K., AND B. J. MARKS. 1995. FRAGSTATS: spatial pattern analysis program for quantifying landscape structure. U. S. For. Serv. Gen Tech. Rept. PNW -351. 
MCGARIGAL, K., AND W. C. MCCOMB. 1995. Relationships between landscape structure and breeding birds in the Oregon Coast Range. Ecological Monographs 65: 235-260.

MILLER, G.W. 1993. Financial aspects of partial cutting practices in central Appalachian hardwoods. U.S. For. Serv. Res. Pap. NE-673.

NETER, J., M.H. KUTNER, C. J. NACHTSHEIM, AND W. WASSERMAN. 1996. Applied Linear Statistical Models. Irwin. Homewood, Illinois. 1181pp.

NICHOLS, J.V. 1996. Effects of two-age timber management and clearcutting on songbird density and reproductive success. M.S. Thesis, West Virginia University. 131pp.

NUR, N., S. L. JONES, AND G. R. GEUPEL. 1999. A statistical guide to data analysis of avian monitoring programs. U.S. Fish and Wildl. Serv. Biol. Tech. Publ. BTP-R60011999.

O'CONNOR. R. J. 1992. Population variation in relation to migrancy status in some North American birds. Pages 64-74 in J. M. Hagan, III and D. W. Johnston, eds., Ecology and conservation of neotropical migrant landbirds. Smithsonian Institution Press, Washington, D.C.

PENDLETON, G. W. 1995. Effects of sampling strategy, detection probability, and independence of counts on the use of point counts. Pages 131-134 in C. Ralph, J. R. Sauer, and S. Droege, eds., Monitoring bird populations by point counts. U.S. For. Serv. Gen. Tech. Rep. PSW-GTR-149.

PETERJOHN, B., J. R. SAUER, AND C. S. ROBBINS. 1995. Population trends from the North American Breeding Bird Survey. Pages 3-19 in T.E. Martin and D. M. Finch, eds., Ecology and management of neotropical migratory birds. Oxford University Press, New York and Oxford.

RALPH, C.J., G.R. GUEPEL, P. PYLE, T.E. MARTIN, AND D.F. DESANTE. 1993. Handbook of field methods for monitoring landbirds. U.S. For. Serv. Gen Tech. Rept. PSW-GTR-144.

ROBINSON, S.K., F.R. THOMPSON III, T.M. DONOVAN, D.R. WHITEHEAD, AND J. FAABORG. 1995. Regional forest fragmentation and the nesting success of migratory birds. Science 267:1987-1990. 
ROSENBERG, K.V., AND J.V. WELLS. 1995. Importance of geographic areas to neotropical migrant birds in the Northeast. U.S.Fish and Wildlife Serv. Rept., Region 5, Hadley, Mass.

SAS INSTITUTE INC. 1991. SAS/STAT Guide for personal computers, version 6 edition. SAS Institute, Inc. Cary, N. Carolina. 1028pp.

SHUGART, H. H., JR., AND D. JAMES. 1973. Ecological succession of breeding bird populations in northwestern Arkansas. Auk 90:62-77.

SMITH, K. 1977. Distribution of summer birds along a forest moisture gradient in an Ozark watershed. Ecology 58:810-819.

STOKES, M. A., C. S. DAVIS, AND G. G. KOCH. 1995. Categorical Data Analysis Using the SAS System. Sas Institute Inc., Cary, North Carolina.

STOUT, S. L., S.B. HORSLEY, AND D.S. DECALESTA. 1992. Assessing plant species diversity: an important challenge in natural resource management. Unpublished report. Forestry Sciences Laboratory, Warren, PA.

STRAUGHSBAUGH, P.D., AND E. L. CORE. 1977. Flora of West Virginia. Seneca Books, Grantsville, West Virginia.

THOMPSON, F. R., III, AND D. E. CAPEN. 1988. Avian assemblages in seral stages of a Vermont forest. Journal of Wildlife Management 52:771-777.

THOMPSON, F. R., III, W. D. DIJAK, T. G. KULOWIEC, AND D A. HAMILTON. 1992. Breeding bird populations in Missouri Ozark Forests with and without clearcutting. Journal of Wildlife Management 56:23-30.

THOMPSON, F.R., III, J.R. PROBST, AND M.G. RAPHAEL. 1995. Impacts of silviculture: overview and management recommendations. Pages 201-219 in T. E. Martin and D. M. Finch, eds., Ecology and management of neotropical migratory birds. Oxford University Press, New York and Oxford.

TILGHMAN, N. G. 1989. Impacts of white-tailed deer on forest regeneration in northwestern Pennsylvania. Journal of Wildlife Management 53:524-532.

WEST VIRGINIA PARTNERS IN FLIGHT. 1999. Neotropical birds that breed in West Virginia. http://www.tracwv.org/wvneo.htm.

WOOD, P.B., J.P. DUGUAY, AND J.V. NICHOLS. 1998. Songbird abundance, nest success, and invertebrate biomass on two-age, clearcut, and unharvested forest stands in the 
Monongahela National Forest, West Virginia. Final Project Rept. West Virg. Coop. Fish and Wildlife Research Unit, Biological Resources Division, USGS, Morgantown, West Virginia.

YAHNER, R. H. 1993. Effects of long-term forest clearcutting on wintering and breeding birds. Wilson Bulletin 105:239-255. 
Table 1.1. Weather and sky code indices used during point count surveys on the Westvaco Wildife and Ecosystem Research Forest, Randolph County, West Virginia, 1996-1998.

\begin{tabular}{ll}
\hline Wind Speed & Sky Cover \\
\hline $0=$ Smoke rises vertically & $0=$ Clear or few clouds \\
$1=$ Wind direction shown by smoke & $1=$ Partly cloudy \\
2 = Wind felt on face, leaves rustle & $2=$ Cloudy or overcast \\
$3=$ Leaves, small twigs in constant motion & $3=$ Fog \\
$4=$ Raises dust and loose paper, small branches move & $4=$ Drizzle \\
$5=$ Small trees in leaf sway & $5=$ Showers \\
\hline
\end{tabular}


Table 1.2. Common names, scientific names, habitat groups, nesting guilds, and migratory strategies of songbird species observed on the Westvaco Wildlife and Ecosystem Research Forest, Randolph County, West Virginia, 1996-1998.

\begin{tabular}{|c|c|c|c|c|}
\hline Species & Scientific Name & Habitat Group & Nesting Guild & Migratory Strategy \\
\hline Acadian flycatcher & Empidonax virescens & Forest-interior & Subcanopy & Neotropical \\
\hline American crow & Corvus brachrynchos & Edge & Canopy & Permanent \\
\hline American goldfinch & Carduelis tristis & Edge & Shrub & Short-distant \\
\hline American redstart & Setophaga ruticilla & Forest-interior & Subcanopy & Neotropical \\
\hline American robin & Turdus migratorius & Edge & Subcanopy & Short-distant \\
\hline Blackburnian warbler & Dendroica fusca & Forest-interior & Canopy & Neotropical \\
\hline Black-and-white warbler & Mniotilta varia & Forest-interior & Ground & Neotropical \\
\hline Black-billed cuckoo & Coccyzus erythropthalmus & Interior-edge & Shrub & Neotropical \\
\hline Black-capped chickadee & Poecile atricapillus & Interior-edge & Cavity & Permanent \\
\hline Black-throated blue warbler & Dendroica caerulescens & Forest-interior & Shrub & Neotropical \\
\hline Black-throated green warbler & Dendroica virens & Forest-interior & Canopy & Neotropical \\
\hline Blue jay & Cyanocitta cristata & Interior-edge & Canopy & Permanent \\
\hline Blue-headed vireo & Vireo solitarius & Forest-interior & Canopy & Neotropical \\
\hline Brown creeper & Certhia americana & Forest-interior & Subcanopy & Permanent \\
\hline Brown-headed cowbird & Molothrus ater & Edge & Parasite & Short-distant \\
\hline Canada warbler & Wilsonia canadensis & Forest-interior & Ground & Neotropical \\
\hline Cedar waxwing & Bombycilla cedrorum & Edge & Subcanopy & Short-distant \\
\hline Cerulean warbler & Dendroica cerulea & Forest-interior & Canopy & Neotropical \\
\hline Chestnut-sided warbler & Dendroica pensylvanica & Edge & Shrub & Neotropical \\
\hline
\end{tabular}


Table 1.2. Continued.

\begin{tabular}{|c|c|c|c|c|}
\hline Species & Scientific Name & Habitat Group & Nesting Guild & Migratory Strategy \\
\hline Common raven & Corvus corax & Forest-interior & Canopy & Permanent \\
\hline Common yellowthroat & Geothlypis trichas & Interior-edge & Ground & Short-distant \\
\hline Dark-eyed junco & Junco hyemalis & Interior-edge & Ground & Permanent \\
\hline Downy woodpecker & Picoides pubescens & Interior-edge & Cavity & Permanent \\
\hline Eastern phoebe & Sayornis phoebe & Edge & Subcanopy & Neotropical \\
\hline Eastern towhee & Pipilo erythrophthalmus & Interior-edge & Ground & Short-distant \\
\hline Eastern wood-pewee & Contopus virens & Interior-edge & Canopy & Neotropical \\
\hline Golden-crowned kinglet & Regulus satrapa & Forest-interior & Canopy & Short-distant \\
\hline Gray catbird & Dumetella carolinensis & Interior-edge & Shrub & Short-distant \\
\hline Hairy woodpecker & Picoides villosus & Forest-interior & Cavity & Permanent \\
\hline Hermit thrush & Catharus guttatus & Forest-interior & Ground & Short-distant \\
\hline Hooded warbler & Wilsonia citrina & Forest-interior & Shrub & Neotropical \\
\hline Indigo bunting & Passerina cyanea & Edge & Shrub & Neotropical \\
\hline Least flycatcher & Empidonax minimus & Edge & Subcanopy & Neotropical \\
\hline Lousiana waterthrush & Seirus motacilla & Forest-interior & Ground & Neotropical \\
\hline Magnolia warbler & Dendroica magnolia & Forest-interior & Subcanopy & Neotropical \\
\hline Mourning warbler & Oporornis philadelphia & Edge & Shrub & Neotropical \\
\hline Nashville warbler & Vermivora ruficapilla & Edge & Ground & Neotropical \\
\hline Northern cardinal & Cardinalis cardinalis & Interior-edge & Shrub & Permanent \\
\hline Northern parula & Parula americana & Interior-edge & Canopy & Neotropical \\
\hline
\end{tabular}


Table 1.2. Continued.

\begin{tabular}{|c|c|c|c|c|}
\hline Species & Scientific Name & Habitat Group & Nesting Guild & Migratory Strategy \\
\hline Northern waterthrush & Seirus noveboracensis & Forest-interior & Ground & Neotropical \\
\hline Ovenbird & Seirus aurocapillus & Forest-interior & Ground & Neotropical \\
\hline Pileated woodpecker & Drycopus pileatus & Forest-interior & Cavity & Permanent \\
\hline Red-breasted nuthatch & Sitta canadensis & Forest-interior & Cavity & Permanent \\
\hline Red-eyed vireo & Vireo olivaceus & Interior-edge & Subcanopy & Neotropical \\
\hline Rose-breasted grosbeak & Pheucticus ludovicianus & Interior-edge & Subcanopy & Neotropical \\
\hline Ruby-throated hummingbird & Archilocus colubris & Interior-edge & Canopy & Neotropical \\
\hline Scarlet tanager & Piranga olivacea & Forest-interior & Canopy & Neotropical \\
\hline Song sparrow & Melospiza melodia & Edge & Shrub & Short-distant \\
\hline Tufted titmouse & Baeolophus bicolor & Interior-edge & Cavity & Permanent \\
\hline Veery & Catharus fuscescens & Forest-interior & Ground & Neotropical \\
\hline Warbling vireo & Vireo gilvus & Edge & Subcanopy & Neotropical \\
\hline White-breasted nuthatch & Sitta carolinensis & Forest-interior & Cavity & Permanent \\
\hline White-eyed vireo & Vireo griseus & Edge & Shrub & Neotropical \\
\hline Winter wren & Troglodytes troglodytes & Forest-interior & Ground & Short-distant \\
\hline Wood thrush & Hylocichla mustelina & Interior-edge & Subcanopy & Neotropical \\
\hline Worm-eating warbler & Helmitheros vermivorus & Forest-interior & Ground & Neotropical \\
\hline Yellow-billed cuckoo & Coccyzus americanus & Interior-edge & Shrub & Neotropical \\
\hline Yellow-shafted flicker & Colaptes auratus & Interior-edge & Cavity & Permanent \\
\hline Yellow-throated vireo & Vireo flavifrons & Interior-edge & Canopy & Neotropical \\
\hline
\end{tabular}


Table 1.3. Landtype classification developed from Westvaco FRISTM data, and landtype groupings for this study, on the Westvaco Wildlife and Ecosystem Research Forest, Randolph County, West Virginia.

\begin{tabular}{llll}
\hline \multicolumn{2}{c}{ Landtype Groupings } & Ridge \\
\hline Submesic Slope & Mesic Slope & Xeric Slope & Broad ridge \\
Very steep & Very steep & Very steep xeric & \\
mesic slope & slope & \\
Steep submesic & Steep mesic & Steep xeric & Narrow ridge or \\
slope & slope & slope & shoulder \\
Moderate & Moderate & Moderate xeric & \\
submesic slope & mesic slope & slope & \\
& & & \\
Stream terrace, & & & \\
bench, or flat & & & \\
\hline
\end{tabular}


Table 1.5. Raptor and gamebird species observed on the Westvaco Wildlife and Ecosystem Research Forest, Randolph County, West Virginia, 1996-1998.

\begin{tabular}{ll}
\hline Species & Scientific Name \\
\hline Red-tailed hawk & Buteo jamaicensis \\
Red-shouldered hawk & Buteo lineatus \\
Broad-winged hawk & Buteo platypterus \\
Northern goshawk & Accipiter gentilis \\
Cooper's hawk & Accipiter cooperii \\
Sharp-shinned hawk & Accipiter striatus \\
Turkey vulture & Cathartes aura \\
Great horned owl & Bubo virginianus \\
Barred owl & Strix varia \\
American woodcock & Scolopax minor \\
Eastern wild turkey & Meleagris gallopavo \\
Ruffed grouse & Bonasa umbellus \\
\hline
\end{tabular}


Table 1.6. Mean abundance per point count, standard error (SE), and 95\% confidence intervals for total songbirds, habitat groups, nesting guilds, and species on the Westvaco Wildlife and Ecosystem Research Forest, Randolph County, West Virginia, 1996-1998.

\begin{tabular}{|c|c|c|c|c|c|c|c|c|c|c|c|c|}
\hline \multirow[b]{3}{*}{ Species/Guild } & \multicolumn{4}{|c|}{1996} & \multicolumn{4}{|c|}{1997} & \multicolumn{4}{|c|}{1998} \\
\hline & \multirow[b]{2}{*}{ Mean } & \multirow[b]{2}{*}{ SE } & \multicolumn{2}{|c|}{$\begin{array}{c}95 \% \text { Confidence } \\
\text { Interval } \\
\end{array}$} & \multirow[b]{2}{*}{ Mean } & \multirow[b]{2}{*}{ SE } & \multicolumn{2}{|c|}{$\begin{array}{c}95 \% \text { Confidence } \\
\text { Interval } \\
\end{array}$} & \multirow[b]{2}{*}{ Mean } & \multirow[b]{2}{*}{ SE } & \multicolumn{2}{|c|}{$\begin{array}{c}95 \% \text { Confidence } \\
\text { Interval } \\
\end{array}$} \\
\hline & & & Lower & Upper & & & Lower & Upper & & & Lower & Upper \\
\hline Total & 13.23 & 0.31 & 12.62 & 13.85 & 11.66 & 0.31 & 11.05 & 12.28 & 12.08 & 0.29 & 11.51 & 12.65 \\
\hline \multicolumn{13}{|l|}{ Habitat Groups: } \\
\hline Interior & 8.47 & 0.25 & 7.97 & 8.97 & 7.40 & 0.22 & 6.98 & 7.83 & 6.92 & 0.22 & 6.49 & 7.36 \\
\hline Interior-edge & 4.46 & 0.17 & 4.12 & 4.80 & 4.17 & 0.18 & 3.82 & 4.52 & 5.00 & 0.14 & 4.71 & 5.29 \\
\hline Edge & 1.40 & 0.13 & 1.15 & 1.65 & 1.03 & 0.10 & 0.82 & 1.23 & 0.99 & 0.12 & 0.75 & 1.22 \\
\hline \multicolumn{13}{|l|}{ Nesting Guilds: } \\
\hline Ground & 4.16 & 0.16 & 3.83 & 4.48 & 4.16 & 0.18 & 3.80 & 4.52 & 3.74 & 0.17 & 3.41 & 4.08 \\
\hline Shrub & 1.60 & 0.14 & 1.33 & 1.88 & 1.13 & 0.09 & 0.94 & 1.32 & 1.29 & 0.10 & 1.09 & 1.50 \\
\hline Subcanopy & 3.53 & 0.15 & 3.22 & 3.83 & 2.62 & 0.10 & 2.41 & 2.82 & 3.94 & 0.11 & 3.72 & 4.17 \\
\hline Canopy & 4.83 & 0.18 & 4.47 & 5.19 & 4.04 & 0.15 & 3.74 & 4.34 & 3.39 & 0.12 & 3.15 & 3.63 \\
\hline Cavity & 1.09 & 0.09 & 0.92 & 1.26 & 1.10 & 0.08 & 0.94 & 1.27 & 1.01 & 0.09 & 0.84 & 1.18 \\
\hline \multicolumn{13}{|l|}{ Species: } \\
\hline Acadian flycatcher & 0.19 & 0.04 & 0.11 & 0.27 & 0.08 & 0.03 & 0.03 & 0.13 & 0.10 & 0.03 & 0.05 & 0.16 \\
\hline American crow & 0.47 & 0.07 & 0.33 & 0.62 & 0.32 & 0.06 & 0.20 & 0.44 & 0.34 & 0.05 & 0.24 & 0.44 \\
\hline American goldfinch & 0.01 & 0.01 & -0.01 & 0.03 & 0.01 & 0.01 & -0.01 & 0.02 & 0.01 & 0.01 & -0.01 & 0.03 \\
\hline American redstart & 0.10 & 0.03 & 0.04 & 0.15 & 0.04 & 0.02 & 0.01 & 0.07 & 0.07 & 0.02 & 0.02 & 0.12 \\
\hline American robin & 0.35 & 0.06 & 0.22 & 0.48 & 0.28 & 0.05 & 0.19 & 0.37 & 0.05 & 0.02 & 0.01 & 0.08 \\
\hline Blackburnian warbler & 0.16 & 0.03 & 0.10 & 0.23 & 0.10 & 0.02 & 0.05 & 0.14 & 0.22 & 0.04 & 0.13 & 0.30 \\
\hline Black-and-white warbler & 0.21 & 0.04 & 0.13 & 0.28 & 0.23 & 0.04 & 0.16 & 0.31 & 0.17 & 0.04 & 0.09 & 0.24 \\
\hline Black-billed cuckoo & 0.00 & 0.00 & 0.00 & 0.00 & 0.00 & 0.00 & 0.00 & 0.00 & 0.01 & 0.01 & -0.01 & 0.03 \\
\hline Black-capped chickadee & 0.54 & 0.06 & 0.42 & 0.66 & 0.57 & 0.07 & 0.43 & 0.71 & 0.51 & 0.06 & 0.39 & 0.62 \\
\hline Black-throated & & & & & & & & & & & & \\
\hline blue warbler & 0.66 & 0.07 & 0.52 & 0.81 & 0.51 & 0.06 & 0.39 & 0.62 & 0.61 & 0.06 & 0.49 & 0.73 \\
\hline Black-throated & & & & & & & & & & & & \\
\hline green warbler & 1.51 & 0.09 & 1.32 & 1.69 & 1.57 & 0.08 & 1.40 & 1.73 & 1.69 & 0.08 & 1.54 & 1.84 \\
\hline Blue jay & 0.77 & 0.08 & 0.61 & 0.94 & 0.56 & 0.06 & 0.45 & 0.68 & 0.38 & 0.05 & 0.28 & 0.49 \\
\hline Blue-headed vireo & 2.03 & 0.11 & 1.81 & 2.24 & 1.60 & 0.08 & 1.44 & 1.77 & 0.54 & 0.06 & 0.42 & 0.66 \\
\hline Brown creeper & 0.01 & 0.01 & -0.01 & 0.03 & 0.03 & 0.02 & 0.00 & 0.06 & 0.11 & 0.03 & 0.05 & 0.17 \\
\hline Brown-headed cowbird & 0.01 & 0.01 & -0.01 & 0.02 & 0.10 & 0.03 & 0.05 & 0.16 & 0.04 & 0.02 & 0.01 & 0.07 \\
\hline
\end{tabular}


Table 1.6. Continued.

\begin{tabular}{|c|c|c|c|c|c|c|c|c|c|c|c|c|}
\hline \multirow[b]{3}{*}{ Species/Guild } & \multicolumn{4}{|c|}{1996} & \multicolumn{4}{|c|}{1997} & \multicolumn{4}{|c|}{1998} \\
\hline & \multirow[b]{2}{*}{ Mean } & \multirow[b]{2}{*}{ SE } & \multicolumn{2}{|c|}{$\begin{array}{l}\text { 95\% Confidence } \\
\text { Interval }\end{array}$} & \multirow[b]{2}{*}{ Mean } & \multirow[b]{2}{*}{ SE } & \multicolumn{2}{|c|}{$\begin{array}{c}\text { 95\% Confidence } \\
\text { Interval }\end{array}$} & \multirow[b]{2}{*}{ Mean } & \multirow[b]{2}{*}{ SE } & \multicolumn{2}{|c|}{$\begin{array}{c}95 \% \text { Confidence } \\
\text { Interval } \\
\end{array}$} \\
\hline & & & Lower & Upper & & & Lower & Upper & & & Lower & Upper \\
\hline Canada warbler & 1.03 & 0.09 & 0.85 & 1.22 & 1.18 & 0.09 & 1.00 & 1.37 & 0.89 & 0.09 & 0.72 & 1.06 \\
\hline Cedar waxwing & 0.01 & 0.01 & -0.01 & 0.04 & 0.00 & 0.00 & 0.00 & 0.00 & 0.01 & 0.01 & -0.01 & 0.02 \\
\hline Cerulean warbler & 0.03 & 0.01 & 0.00 & 0.05 & 0.03 & 0.01 & 0.00 & 0.05 & 0.01 & 0.01 & -0.01 & 0.02 \\
\hline Chestnut-sided warbler & 0.46 & 0.07 & 0.33 & 0.59 & 0.29 & 0.05 & 0.20 & 0.39 & 0.28 & 0.05 & 0.17 & 0.38 \\
\hline Common raven & 0.08 & 0.03 & 0.01 & 0.15 & 0.14 & 0.04 & 0.07 & 0.21 & 0.03 & 0.02 & 0.00 & 0.06 \\
\hline Common yellowthroat & 0.00 & 0.00 & 0.00 & 0.00 & 0.01 & 0.01 & -0.01 & 0.02 & 0.00 & 0.00 & 0.00 & 0.00 \\
\hline Dark-eyed junco & 0.78 & 0.08 & 0.63 & 0.93 & 1.08 & 0.09 & 0.90 & 1.25 & 1.01 & 0.08 & 0.87 & 1.16 \\
\hline Downy woodpecker & 0.05 & 0.02 & 0.00 & 0.10 & 0.09 & 0.02 & 0.04 & 0.14 & 0.07 & 0.02 & 0.03 & 0.11 \\
\hline Eastern phoebe & 0.01 & 0.01 & -0.01 & 0.02 & 0.01 & 0.01 & -0.01 & 0.02 & 0.03 & 0.01 & 0.00 & 0.05 \\
\hline Eastern towhee & 0.45 & 0.06 & 0.32 & 0.57 & 0.41 & 0.06 & 0.29 & 0.53 & 0.31 & 0.05 & 0.21 & 0.41 \\
\hline Eastern wood-pewee & 0.08 & 0.02 & 0.03 & 0.13 & 0.05 & 0.02 & 0.02 & 0.09 & 0.04 & 0.02 & 0.01 & 0.07 \\
\hline Golden-crowned kinglet & 0.03 & 0.02 & 0.00 & 0.06 & 0.16 & 0.04 & 0.08 & 0.23 & 0.03 & 0.01 & 0.00 & 0.05 \\
\hline Gray catbird & 0.05 & 0.04 & -0.03 & 0.14 & 0.01 & 0.01 & -0.01 & 0.02 & 0.03 & 0.01 & 0.00 & 0.05 \\
\hline Hairy woodpecker & 0.12 & 0.03 & 0.06 & 0.17 & 0.16 & 0.03 & 0.09 & 0.23 & 0.17 & 0.04 & 0.09 & 0.25 \\
\hline Hermit thrush & 0.22 & 0.04 & 0.13 & 0.30 & 0.29 & 0.05 & 0.20 & 0.39 & 0.42 & 0.05 & 0.32 & 0.52 \\
\hline Hooded warbler & 0.26 & 0.06 & 0.15 & 0.37 & 0.25 & 0.04 & 0.17 & 0.34 & 0.21 & 0.04 & 0.12 & 0.29 \\
\hline Indigo bunting & 0.25 & 0.05 & 0.15 & 0.35 & 0.16 & 0.04 & 0.08 & 0.25 & 0.26 & 0.05 & 0.16 & 0.36 \\
\hline Least flycatcher & 0.00 & 0.00 & 0.00 & 0.00 & 0.01 & 0.01 & -0.01 & 0.03 & 0.00 & 0.00 & 0.00 & 0.00 \\
\hline Louisiana waterthrush & 0.01 & 0.01 & -0.01 & 0.02 & 0.01 & 0.01 & -0.01 & 0.03 & 0.01 & 0.01 & -0.01 & 0.03 \\
\hline Magnolia warbler & 0.79 & 0.08 & 0.63 & 0.96 & 0.36 & 0.05 & 0.26 & 0.47 & 0.74 & 0.08 & 0.59 & 0.89 \\
\hline Mourning warbler & 0.01 & 0.01 & -0.01 & 0.02 & 0.01 & 0.01 & -0.01 & 0.02 & 0.01 & 0.01 & -0.01 & 0.02 \\
\hline Nashville warbler & 0.01 & 0.01 & -0.01 & 0.03 & 0.00 & 0.00 & 0.00 & 0.00 & 0.01 & 0.01 & -0.01 & 0.02 \\
\hline Northern cardinal & 0.01 & 0.01 & -0.01 & 0.02 & 0.01 & 0.01 & -0.01 & 0.03 & 0.01 & 0.01 & -0.01 & 0.02 \\
\hline Northern parula & 0.01 & 0.01 & -0.01 & 0.03 & 0.02 & 0.01 & 0.00 & 0.04 & 0.01 & 0.01 & -0.01 & 0.03 \\
\hline Northern waterthrush & 0.01 & 0.01 & -0.01 & 0.02 & 0.00 & 0.00 & 0.00 & 0.00 & 0.00 & 0.00 & 0.00 & 0.00 \\
\hline Ovenbird & 0.52 & 0.06 & 0.40 & 0.64 & 0.39 & 0.06 & 0.28 & 0.50 & 0.37 & 0.05 & 0.27 & 0.47 \\
\hline Pileated woodpecker & 0.32 & 0.04 & 0.23 & 0.40 & 0.24 & 0.04 & 0.17 & 0.31 & 0.17 & 0.03 & 0.11 & 0.23 \\
\hline Red-breasted nuthatch & 0.08 & 0.02 & 0.03 & 0.12 & 0.02 & 0.01 & 0.00 & 0.04 & 0.06 & 0.02 & 0.02 & 0.11 \\
\hline Red-eyed vireo & 1.94 & 0.10 & 1.75 & 2.13 & 1.71 & 0.08 & 1.54 & 1.87 & 2.70 & 0.08 & 2.55 & 2.85 \\
\hline Rose-breasted grosbeak & 0.40 & 0.05 & 0.30 & 0.50 & 0.32 & 0.04 & 0.24 & 0.41 & 0.21 & 0.04 & 0.14 & 0.28 \\
\hline
\end{tabular}


Table 1.6. Continued.

\begin{tabular}{|c|c|c|c|c|c|c|c|c|c|c|c|c|}
\hline \multirow[b]{3}{*}{ Species/Guild } & \multicolumn{4}{|c|}{1996} & \multicolumn{4}{|c|}{1997} & \multicolumn{4}{|c|}{1998} \\
\hline & \multirow[b]{2}{*}{ Mean } & \multirow[b]{2}{*}{ SE } & \multicolumn{2}{|c|}{$\begin{array}{c}95 \% \text { Confidence } \\
\text { Interval }\end{array}$} & \multirow[b]{2}{*}{ Mean } & \multirow[b]{2}{*}{$\mathrm{SE}$} & \multicolumn{2}{|c|}{$\begin{array}{l}95 \% \text { Confidence } \\
\text { Interval }\end{array}$} & \multirow[b]{2}{*}{ Mean } & \multirow[b]{2}{*}{$\mathrm{SE}$} & \multicolumn{2}{|c|}{$\begin{array}{c}95 \% \text { Confidence } \\
\text { Interval }\end{array}$} \\
\hline & & & Lower & Upper & & & Lower & Upper & & & Lower & Upper \\
\hline Ruby-throated & & & & & & & & & & & & \\
\hline Hummingbird & 0.00 & 0.00 & 0.00 & 0.00 & 0.01 & 0.01 & -0.01 & 0.02 & 0.00 & 0.00 & 0.00 & 0.00 \\
\hline Scarlet tanager & 0.65 & 0.06 & 0.53 & 0.77 & 0.48 & 0.04 & 0.40 & 0.56 & 0.70 & 0.05 & 0.60 & 0.80 \\
\hline Song sparrow & 0.01 & 0.01 & -0.01 & 0.02 & 0.01 & 0.01 & -0.01 & 0.02 & 0.01 & 0.01 & -0.01 & 0.03 \\
\hline Tufted titmouse & 0.01 & 0.01 & -0.01 & 0.02 & 0.03 & 0.02 & 0.00 & 0.06 & 0.01 & 0.01 & -0.01 & 0.03 \\
\hline Veery & 1.31 & 0.08 & 1.15 & 1.47 & 0.90 & 0.07 & 0.77 & 1.03 & 0.90 & 0.07 & 0.77 & 1.04 \\
\hline Warbling vireo & 0.00 & 0.00 & 0.00 & 0.00 & 0.01 & 0.01 & -0.01 & 0.02 & 0.00 & 0.00 & 0.00 & 0.00 \\
\hline White-breasted nuthatch & 0.04 & 0.02 & 0.01 & 0.07 & 0.10 & 0.03 & 0.05 & 0.16 & 0.08 & 0.02 & 0.03 & 0.12 \\
\hline White-eyed vireo & 0.01 & 0.01 & -0.01 & 0.02 & 0.00 & 0.00 & 0.00 & 0.00 & 0.00 & 0.00 & 0.00 & 0.00 \\
\hline Winter wren & 0.44 & 0.05 & 0.34 & 0.53 & 0.50 & 0.05 & 0.41 & 0.59 & 0.50 & 0.05 & 0.41 & 0.59 \\
\hline Wood thrush & 0.41 & 0.06 & 0.30 & 0.52 & 0.12 & 0.03 & 0.07 & 0.18 & 0.28 & 0.04 & 0.19 & 0.37 \\
\hline Worm-eating warbler & 0.05 & 0.02 & 0.01 & 0.08 & 0.02 & 0.01 & 0.00 & 0.04 & 0.00 & 0.00 & 0.00 & 0.00 \\
\hline Yellow-billed cuckoo & 0.01 & 0.01 & -0.01 & 0.03 & 0.04 & 0.02 & 0.01 & 0.07 & 0.01 & 0.01 & -0.01 & 0.03 \\
\hline Yellow-shafted flicker & 0.03 & 0.02 & 0.00 & 0.06 & 0.03 & 0.02 & 0.00 & 0.06 & 0.10 & 0.02 & 0.05 & 0.15 \\
\hline Yellow-throated vireo & 0.05 & 0.02 & 0.01 & 0.08 & 0.01 & 0.01 & -0.01 & 0.03 & 0.04 & 0.02 & 0.01 & 0.07 \\
\hline
\end{tabular}


Table 1.7. Total abundance and percent frequency of occurrence of total songbirds, habitat groups, nesting guilds, and species on the Westvaco Wildlife and Ecosystem Research Forest, Randolph County, West Virginia, 1996-1998.

\begin{tabular}{|c|c|c|c|c|c|c|}
\hline \multirow[b]{2}{*}{ Species/Guild } & \multicolumn{2}{|c|}{1996} & \multicolumn{2}{|c|}{1997} & \multicolumn{2}{|c|}{1998} \\
\hline & $\begin{array}{c}\text { Total } \\
\text { Abundance }\end{array}$ & $\begin{array}{c}\text { Freq. of } \\
\text { Occurrence }\end{array}$ & $\begin{array}{c}\text { Total } \\
\text { Abundance }\end{array}$ & $\begin{array}{c}\text { Freq. of } \\
\text { Occurrence }\end{array}$ & $\begin{array}{c}\text { Total } \\
\text { Abundance }\end{array}$ & $\begin{array}{c}\text { Freq. of } \\
\text { Occurrence }\end{array}$ \\
\hline Total & 1932 & 100.0 & 1703 & 100.0 & 1739 & 100.0 \\
\hline \multicolumn{7}{|l|}{ Habitat Groups: } \\
\hline Interior & 1237 & 100.0 & 1081 & 100.0 & 997 & 98.6 \\
\hline Interior-edge & 651 & 98.6 & 609 & 97.9 & 720 & 100.0 \\
\hline Edge & 204 & 64.4 & 150 & 56.2 & 142 & 50.0 \\
\hline \multicolumn{7}{|l|}{ Nesting Guilds: } \\
\hline Ground & 607 & 98.6 & 607 & 97.9 & 539 & 98.6 \\
\hline Shrub & 234 & 72.6 & 165 & 62.3 & 186 & 69.4 \\
\hline Subcanopy & 515 & 98.6 & 382 & 97.9 & 568 & 100.0 \\
\hline Canopy & 705 & 98.6 & 590 & 97.3 & 488 & 97.9 \\
\hline Cavity & 159 & 67.8 & 161 & 70.5 & 146 & 68.8 \\
\hline \multicolumn{7}{|l|}{ Species: } \\
\hline Acadian flycatcher & 28 & 15.1 & 12 & 6.8 & 15 & 9.0 \\
\hline American crow $^{\mathrm{a}}$ & 69 & 28.8 & 47 & 21.2 & 49 & 27.8 \\
\hline American goldfinch & 2 & 1.4 & 1 & 0.7 & 2 & 1.4 \\
\hline American redstart & 14 & 8.9 & 6 & 4.1 & 10 & 6.3 \\
\hline American robin & 51 & 20.5 & 41 & 23.3 & 7 & 4.9 \\
\hline Blackburnian warbler & 24 & 16.4 & 14 & 9.6 & 31 & 17.4 \\
\hline Black-and-white warbler & 30 & 17.8 & 34 & 21.9 & 24 & 14.6 \\
\hline Black-billed cuckoo & 0 & 0.0 & 0 & 0.0 & 2 & 1.4 \\
\hline Black-capped chickadee $^{\mathrm{a}}$ & 79 & 41.1 & 83 & 41.8 & 73 & 41.7 \\
\hline Black-throated blue warbler ${ }^{\mathrm{a}}$ & 97 & 43.8 & 74 & 39.7 & 88 & 47.9 \\
\hline Black-throated green warbler ${ }^{a}$ & 220 & 80.1 & 229 & 84.9 & 243 & 91.7 \\
\hline Blue jay $^{\mathrm{a}}$ & 113 & 49.3 & 82 & 45.2 & 55 & 31.3 \\
\hline Blue-headed vireo $^{a}$ & 296 & 89.7 & 234 & 85.6 & 78 & 42.4 \\
\hline Brown creeper & 2 & 1.4 & 5 & 3.4 & 16 & 9.7 \\
\hline Brown-headed cowbird & 1 & 0.7 & 15 & 9.6 & 6 & 4.2 \\
\hline Canada warbler ${ }^{\mathrm{a}}$ & 151 & 57.5 & 173 & 65.8 & 128 & 54.2 \\
\hline Cedar waxwing & 2 & 0.7 & 0 & 0.0 & 1 & 0.7 \\
\hline
\end{tabular}


Table 1.7. Continued.

\begin{tabular}{|c|c|c|c|c|c|c|}
\hline \multirow[b]{2}{*}{ Species/Guild } & \multicolumn{2}{|c|}{1996} & \multicolumn{2}{|c|}{1997} & \multicolumn{2}{|c|}{1998} \\
\hline & $\begin{array}{c}\text { Total } \\
\text { Abundance }\end{array}$ & $\begin{array}{c}\text { Freq. of } \\
\text { Occurrence }\end{array}$ & $\begin{array}{c}\text { Total } \\
\text { Abundance }\end{array}$ & $\begin{array}{c}\text { Freq. of } \\
\text { Occurrence }\end{array}$ & $\begin{array}{c}\text { Total } \\
\text { Abundance }\end{array}$ & $\begin{array}{c}\text { Freq. of } \\
\text { Occurrence }\end{array}$ \\
\hline Cerulean warbler & 4 & 2.7 & 4 & 2.7 & 1 & 0.7 \\
\hline Chestnut-sided warbler & 67 & 31.5 & 43 & 23.3 & 40 & 18.8 \\
\hline Common raven & 12 & 5.5 & 20 & 11.0 & 5 & 3.5 \\
\hline Common yellowthroat & 0 & 0.0 & 1 & 0.7 & 0 & 0.0 \\
\hline Dark-eyed junco $^{\mathrm{a}}$ & 114 & 52.7 & 157 & 63.0 & 146 & 66.7 \\
\hline Downy woodpecker & 7 & 3.4 & 13 & 8.9 & 10 & 6.9 \\
\hline Eastern phoebe & 1 & 0.7 & 1 & 0.7 & 4 & 2.8 \\
\hline Eastern towhee $^{\mathrm{a}}$ & 65 & 32.2 & 60 & 28.8 & 45 & 24.3 \\
\hline Eastern wood-pewee & 12 & 7.5 & 8 & 5.5 & 6 & 4.2 \\
\hline Golden-crowned kinglet & 5 & 3.4 & 23 & 12.3 & 4 & 2.8 \\
\hline Gray catbird & 8 & 2.1 & 1 & 0.7 & 4 & 2.8 \\
\hline Hairy woodpecker & 17 & 11.0 & 23 & 13.7 & 25 & 13.2 \\
\hline Hermit thrush & 32 & 17.8 & 43 & 24.0 & 61 & 36.1 \\
\hline Hooded warbler & 38 & 17.8 & 37 & 21.2 & 30 & 16.7 \\
\hline Indigo bunting & 37 & 18.5 & 24 & 12.3 & 37 & 18.1 \\
\hline Least flycatcher & 0 & 0.0 & 2 & 1.4 & 0 & 0.0 \\
\hline Louisiana waterthrush & 1 & 0.7 & 2 & 1.4 & 2 & 1.4 \\
\hline Magnolia warbler ${ }^{\mathrm{a}}$ & 116 & 47.9 & 53 & 27.4 & 106 & 47.9 \\
\hline Mourning warbler & 1 & 0.7 & 1 & 0.7 & 1 & 0.7 \\
\hline Nashville warbler & 2 & 1.4 & 0 & 0.0 & 1 & 0.7 \\
\hline Northern cardinal & 1 & 0.7 & 2 & 1.4 & 1 & 0.7 \\
\hline Northern parula & 2 & 1.4 & 3 & 2.1 & 2 & 1.4 \\
\hline Northern waterthrush & 1 & 0.7 & 0 & 0.0 & 0 & 0.0 \\
\hline Ovenbird $^{\mathrm{a}}$ & 76 & 37.7 & 57 & 28.8 & 53 & 29.9 \\
\hline Pileated woodpecker & 46 & 28.1 & 35 & 23.3 & 24 & 16.7 \\
\hline Red-breasted nuthatch & 11 & 6.8 & 3 & 2.1 & 9 & 5.6 \\
\hline Red-eyed vireo ${ }^{\mathrm{a}}$ & 283 & 91.8 & 249 & 87.0 & 389 & 100.0 \\
\hline Rose-breasted grosbeak ${ }^{\mathrm{a}}$ & 58 & 33.6 & 47 & 29.5 & 30 & 20.1 \\
\hline Ruby-throated hummingbird & 0 & 0.0 & 1 & 0.7 & 0 & 0.0 \\
\hline Scarlet tanager ${ }^{\mathrm{a}}$ & 95 & 54.8 & 70 & 47.3 & 101 & 63.2 \\
\hline Song sparrow & 1 & 0.7 & 1 & 0.7 & 2 & 1.4 \\
\hline & & 39 & & & & \\
\hline
\end{tabular}


Table 1.7. Continued.

\begin{tabular}{|c|c|c|c|c|c|c|}
\hline \multirow[b]{2}{*}{ Species/Guild } & \multicolumn{2}{|c|}{1996} & \multicolumn{2}{|c|}{1997} & \multicolumn{2}{|c|}{1998} \\
\hline & $\begin{array}{c}\text { Total } \\
\text { Abundance }\end{array}$ & $\begin{array}{c}\text { Freq. of } \\
\text { Occurrence }\end{array}$ & $\begin{array}{c}\text { Total } \\
\text { Abundance } \\
\end{array}$ & $\begin{array}{c}\text { Freq. of } \\
\text { Occurrence }\end{array}$ & $\begin{array}{c}\text { Total } \\
\text { Abundance } \\
\end{array}$ & $\begin{array}{c}\text { Freq. of } \\
\text { Occurrence }\end{array}$ \\
\hline Tufted titmouse & 1 & 0.7 & 5 & 3.4 & 2 & 1.4 \\
\hline Veery $^{\mathrm{a}}$ & 191 & 76.7 & 132 & 66.4 & 130 & 65.3 \\
\hline Warbling vireo & 0 & 0.0 & 1 & 0.7 & 0 & 0.0 \\
\hline White-breasted nuthatch & 6 & 4.1 & 15 & 9.6 & 11 & 6.9 \\
\hline White-eyed vireo & 1 & 0.7 & 0 & 0.0 & 0 & 0.0 \\
\hline Winter wren $^{\mathrm{a}}$ & 64 & 39.7 & 73 & 45.9 & 72 & 47.2 \\
\hline Wood thrush & 60 & 31.5 & 18 & 12.3 & 40 & 23.6 \\
\hline Worm-eating warbler & 7 & 4.8 & 3 & 2.1 & 0 & 0.0 \\
\hline Yellow-billed cuckoo & 2 & 1.4 & 6 & 4.1 & 2 & 1.4 \\
\hline Yellow-shafted flicker & 5 & 3.4 & 5 & 3.4 & 14 & 9.7 \\
\hline Yellow-throated vireo & 7 & 4.8 & 2 & 1.4 & 6 & 4.2 \\
\hline
\end{tabular}

${ }^{a}$ Species observed at $\geq 20 \%$ of counts each year and used in subsequent analyses 
Table 1.8. ANOVA and Scheffe' multiple comparison test results comparing songbird abundance of species observed at $\geq 20 \%$ of point counts among years on the Westvaco Wildlife and Ecosystem Research Forest, Randolph County, West Virginia, 1996-1998. Years with the same letter do not differ at the family alpha level of 0.10 . "A" indicates highest abundance, followed by "B" and "C".

\begin{tabular}{lrrrrr}
\hline & & & \multicolumn{3}{c}{ Year } \\
\cline { 4 - 7 } Species & \multicolumn{2}{c}{$\begin{array}{c}\text { P- } \\
\text { value }\end{array}$} & 1996 & 1997 & 1998 \\
\hline Edge Species: & & & & & \\
\hline American crow & 0.99 & 0.37 & & & \\
Interior-edge Species: & & & & & \\
Black-capped chickadee & 0.47 & 0.62 & & & \\
Blue jay & 4.80 & $<0.01$ & $\mathrm{~A}$ & $\mathrm{AB}$ & $\mathrm{B}$ \\
Dark-eyed junco & 1.64 & 0.19 & & & \\
Eastern towhee & 4.43 & 0.01 & $\mathrm{~A}$ & $\mathrm{AB}$ & $\mathrm{B}$ \\
Red-eyed vireo & 29.65 & $<0.01$ & $\mathrm{~B}$ & $\mathrm{~B}$ & $\mathrm{~A}$ \\
Rose-breasted grosbeak & 5.47 & $<0.01$ & $\mathrm{~A}$ & $\mathrm{AB}$ & $\mathrm{B}$ \\
Interior Species: & & & & & \\
Black-throated blue warbler & 1.30 & 0.27 & & & \\
Black-throated green warbler & 4.44 & 0.01 & $\mathrm{~B}$ & $\mathrm{AC}$ & $\mathrm{A}$ \\
Blue-headed vireo & 80.30 & $<0.01$ & $\mathrm{~A}$ & $\mathrm{~B}$ & $\mathrm{C}$ \\
Canada warbler & 1.93 & 0.15 & & & \\
Magnolia warbler & 9.82 & $<0.01$ & $\mathrm{~A}$ & $\mathrm{~B}$ & $\mathrm{~A}$ \\
Ovenbird & 0.66 & 0.52 & & & \\
Scarlet tanager & 5.15 & $<0.01$ & $\mathrm{AB}$ & $\mathrm{B}$ & $\mathrm{A}$ \\
Veery & 4.77 & $<0.01$ & $\mathrm{~A}$ & $\mathrm{~B}$ & $\mathrm{~B}$ \\
Winter wren & 0.08 & 0.92 & & & \\
\hline
\end{tabular}


Table 1.9. Means and standard errors of total songbird abundance, abundance of habitat groups and nesting guilds, and of individual species observed at $\geq 20 \%$ of point counts on submesic $(n=25)$, mesic $(n=29)$, and xeric slopes $(n=17)$, and ridges $(n=39)$ at unharvested point counts on theWestvaco Wildlife and Ecosystem Research Forest, Randolph County, West Virginia, 1996-1998. Means are for untransformed data.

\begin{tabular}{|c|c|c|c|c|c|c|c|c|c|c|c|c|}
\hline \multirow[b]{3}{*}{ Species/Guild } & \multirow{2}{*}{\multicolumn{4}{|c|}{$\begin{array}{l}\frac{1996}{\text { Mean }} \\
(\mathrm{SE})\end{array}$}} & \multirow{2}{*}{\multicolumn{4}{|c|}{$\begin{array}{c}1997 \\
\text { Mean } \\
(\mathrm{SE})\end{array}$}} & \multirow{2}{*}{\multicolumn{4}{|c|}{$\begin{array}{c}1998 \\
\text { Mean } \\
(\mathrm{SE})\end{array}$}} \\
\hline & & & & & & & & & & & & \\
\hline & $\begin{array}{c}\text { Submesic } \\
\text { Slope }\end{array}$ & $\begin{array}{l}\text { Mesic } \\
\text { Slope }\end{array}$ & $\begin{array}{l}\text { Xeric } \\
\text { Slope }\end{array}$ & Ridge & $\begin{array}{c}\text { Submesic } \\
\text { Slope }\end{array}$ & $\begin{array}{l}\text { Mesic } \\
\text { Slope }\end{array}$ & $\begin{array}{l}\text { Xeric } \\
\text { Slope } \\
\end{array}$ & Ridge & $\begin{array}{c}\text { Submesic } \\
\text { Slope }\end{array}$ & $\begin{array}{l}\text { Mesic } \\
\text { Slope } \\
\end{array}$ & $\begin{array}{l}\text { Xeric } \\
\text { Slope } \\
\end{array}$ & Ridge \\
\hline Total & $\begin{array}{l}13.81 \\
(0.81)\end{array}$ & $\begin{array}{l}11.86 \\
(0.59)\end{array}$ & $\begin{array}{l}12.56 \\
(0.89)\end{array}$ & $\begin{array}{r}13.13 \\
(0.64)\end{array}$ & $\begin{array}{l}11.35 \\
(0.70)\end{array}$ & $\begin{array}{l}10.79 \\
(0.61)\end{array}$ & $\begin{array}{l}10.33 \\
(0.76)\end{array}$ & $\begin{array}{l}11.03 \\
(0.49)\end{array}$ & $\begin{array}{l}12.48 \\
(0.75)\end{array}$ & $\begin{array}{l}10.66 \\
(0.57)\end{array}$ & $\begin{array}{l}11.29 \\
(0.65)\end{array}$ & $\begin{array}{l}11.05 \\
(0.38)\end{array}$ \\
\hline \multicolumn{13}{|l|}{ Habitat Groups: } \\
\hline Interior & $\begin{array}{c}9.00 \\
(0.68)\end{array}$ & $\begin{array}{c}7.48 \\
(0.45)\end{array}$ & $\begin{array}{c}8.33 \\
(0.75)\end{array}$ & $\begin{array}{c}8.85 \\
(0.53)\end{array}$ & $\begin{array}{c}7.31 \\
(0.58)\end{array}$ & $\begin{array}{c}6.59 \\
(0.50)\end{array}$ & $\begin{array}{c}7.22 \\
(0.60)\end{array}$ & $\begin{array}{c}7.72 \\
(0.35)\end{array}$ & $\begin{array}{c}7.16 \\
(0.78)\end{array}$ & $\begin{array}{c}6.31 \\
(0.46)\end{array}$ & $\begin{array}{c}7.12 \\
(0.61)\end{array}$ & $\begin{array}{c}6.90 \\
(0.28)\end{array}$ \\
\hline Interior-edge & $\begin{array}{c}4.62 \\
(0.46)\end{array}$ & $\begin{array}{c}4.24 \\
(0.41)\end{array}$ & $\begin{array}{c}4.28 \\
(0.41)\end{array}$ & $\begin{array}{c}4.05 \\
(0.30)\end{array}$ & $\begin{array}{c}4.04 \\
(0.31)\end{array}$ & $\begin{array}{c}4.14 \\
(0.33)\end{array}$ & $\begin{array}{c}3.44 \\
(0.47)\end{array}$ & $\begin{array}{c}3.28 \\
(0.28)\end{array}$ & $\begin{array}{c}5.08 \\
(0.30)\end{array}$ & $\begin{array}{c}4.34 \\
(0.19)\end{array}$ & $\begin{array}{c}4.47 \\
(0.40)\end{array}$ & $\begin{array}{c}4.54 \\
(0.24)\end{array}$ \\
\hline Edge & $\begin{array}{c}1.54 \\
(0.30)\end{array}$ & $\begin{array}{c}1.17 \\
(0.21)\end{array}$ & $\begin{array}{c}1.06 \\
(0.30)\end{array}$ & $\begin{array}{c}1.05 \\
(0.19)\end{array}$ & $\begin{array}{c}0.96 \\
(0.20)\end{array}$ & $\begin{array}{c}1.14 \\
(0.25)\end{array}$ & $\begin{array}{c}0.89 \\
(0.23)\end{array}$ & $\begin{array}{c}0.67 \\
(0.14)\end{array}$ & $\begin{array}{c}0.72 \\
(0.23)\end{array}$ & $\begin{array}{c}1.21 \\
(0.27)\end{array}$ & $\begin{array}{c}0.76 \\
(0.24)\end{array}$ & $\begin{array}{c}0.44 \\
(0.13)\end{array}$ \\
\hline \multicolumn{13}{|l|}{ Nesting Guilds: } \\
\hline Ground & $\begin{array}{c}4.19 \\
(0.37)\end{array}$ & $\begin{array}{c}3.76 \\
(0.38)\end{array}$ & $\begin{array}{c}4.89 \\
(0.49)\end{array}$ & $\begin{array}{l}3.90 \\
0.33\end{array}$ & $\begin{array}{c}4.04 \\
(0.36)\end{array}$ & $\begin{array}{c}3.48 \\
(0.40)\end{array}$ & $\begin{array}{c}4.28 \\
(0.43)\end{array}$ & $\begin{array}{c}3.69 \\
(0.31)\end{array}$ & $\begin{array}{c}3.96 \\
(0.48)\end{array}$ & $\begin{array}{c}2.97 \\
(0.37)\end{array}$ & $\begin{array}{c}4.12 \\
(0.53)\end{array}$ & $\begin{array}{c}3.10 \\
(0.25)\end{array}$ \\
\hline Shrub & $\begin{array}{c}1.50 \\
(0.27)\end{array}$ & $\begin{array}{c}1.45 \\
(0.22)\end{array}$ & $\begin{array}{c}1.83 \\
(0.37)\end{array}$ & $\begin{array}{l}1.38 \\
0.25\end{array}$ & $\begin{array}{c}1.08 \\
(0.16)\end{array}$ & $\begin{array}{c}1.10 \\
(0.20)\end{array}$ & $\begin{array}{c}1.06 \\
(0.25)\end{array}$ & $\begin{array}{c}1.15 \\
(0.20)\end{array}$ & $\begin{array}{c}0.92 \\
(0.18)\end{array}$ & $\begin{array}{c}1.31 \\
(0.22)\end{array}$ & $\begin{array}{c}1.24 \\
(0.25)\end{array}$ & $\begin{array}{c}1.03 \\
(0.15)\end{array}$ \\
\hline Subcanopy & $\begin{array}{c}3.35 \\
(0.31)\end{array}$ & $\begin{array}{c}3.34 \\
(0.33)\end{array}$ & $\begin{array}{c}3.72 \\
(0.50)\end{array}$ & $\begin{array}{l}3.49 \\
0.30\end{array}$ & $\begin{array}{c}2.58 \\
(0.24)\end{array}$ & $\begin{array}{c}2.79 \\
(0.26)\end{array}$ & $\begin{array}{c}2.72 \\
(0.30)\end{array}$ & $\begin{array}{c}2.26 \\
(0.18)\end{array}$ & $\begin{array}{c}4.36 \\
(0.26)\end{array}$ & $\begin{array}{c}3.79 \\
(0.23)\end{array}$ & $\begin{array}{c}4.00 \\
(0.38)\end{array}$ & $\begin{array}{c}3.69 \\
(0.21)\end{array}$ \\
\hline Canopy & $\begin{array}{c}5.81 \\
(0.46)\end{array}$ & $\begin{array}{c}4.24 \\
(0.28)\end{array}$ & $\begin{array}{c}3.28 \\
(0.46)\end{array}$ & $\begin{array}{l}4.97 \\
0.35\end{array}$ & $\begin{array}{c}4.12 \\
(0.30)\end{array}$ & $\begin{array}{c}3.62 \\
(0.30)\end{array}$ & $\begin{array}{c}2.89 \\
(0.41)\end{array}$ & $\begin{array}{c}4.15 \\
(0.29)\end{array}$ & $\begin{array}{c}3.44 \\
(0.37)\end{array}$ & $\begin{array}{c}3.55 \\
(0.27)\end{array}$ & $\begin{array}{c}2.65 \\
(0.27)\end{array}$ & $\begin{array}{c}3.54 \\
(0.23)\end{array}$ \\
\hline Cavity & $\begin{array}{c}1.04 \\
(0.19)\end{array}$ & $\begin{array}{c}1.03 \\
(0.26)\end{array}$ & $\begin{array}{c}0.94 \\
(0.22)\end{array}$ & $\begin{array}{l}1.15 \\
0.14\end{array}$ & $\begin{array}{c}1.04 \\
(0.23)\end{array}$ & $\begin{array}{c}1.10 \\
(0.15)\end{array}$ & $\begin{array}{c}0.61 \\
(0.18)\end{array}$ & $\begin{array}{c}0.97 \\
(0.13)\end{array}$ & $\begin{array}{c}1.00 \\
(0.24)\end{array}$ & $\begin{array}{c}0.59 \\
(0.14)\end{array}$ & $\begin{array}{c}1.00 \\
(0.21)\end{array}$ & $\begin{array}{c}0.97 \\
(0.12)\end{array}$ \\
\hline \multicolumn{13}{|l|}{ Species: } \\
\hline American crow & $\begin{array}{c}0.77 \\
(0.26)\end{array}$ & $\begin{array}{c}0.41 \\
(0.14)\end{array}$ & $\begin{array}{c}0.33 \\
(0.16)\end{array}$ & $\begin{array}{c}0.31 \\
(0.10)\end{array}$ & $\begin{array}{c}0.35 \\
(0.14)\end{array}$ & $\begin{array}{c}0.45 \\
(0.17)\end{array}$ & $\begin{array}{c}0.33 \\
(0.16)\end{array}$ & $\begin{array}{c}0.15 \\
(0.07)\end{array}$ & $\begin{array}{c}0.36 \\
(0.11)\end{array}$ & $\begin{array}{c}0.55 \\
(0.15)\end{array}$ & $\begin{array}{c}0.53 \\
(0.21)\end{array}$ & $\begin{array}{c}0.18 \\
(0.07)\end{array}$ \\
\hline
\end{tabular}


Table 1.9. Continued.

\begin{tabular}{|c|c|c|c|c|c|c|c|c|c|c|c|c|}
\hline \multirow[b]{3}{*}{ Species/Guild } & \multirow{2}{*}{\multicolumn{4}{|c|}{$\begin{array}{c}1996 \\
\text { Mean } \\
(\mathrm{SE})\end{array}$}} & \multirow{2}{*}{\multicolumn{4}{|c|}{$\begin{array}{l}1997 \\
\text { Mean } \\
(\mathrm{SE})\end{array}$}} & \multirow{2}{*}{\multicolumn{4}{|c|}{$\begin{array}{c}1998 \\
\text { Mean } \\
(\mathrm{SE})\end{array}$}} \\
\hline & & & & & & & & & & & & \\
\hline & $\begin{array}{l}\text { Submesic } \\
\text { Slope }\end{array}$ & $\begin{array}{l}\text { Mesic } \\
\text { Slope } \\
\end{array}$ & $\begin{array}{l}\text { Xeric } \\
\text { Slope } \\
\end{array}$ & Ridge & $\begin{array}{l}\text { Submesic } \\
\text { Slope } \\
\end{array}$ & $\begin{array}{l}\text { Mesic } \\
\text { Slope } \\
\end{array}$ & $\begin{array}{l}\text { Xeric } \\
\text { Slope } \\
\end{array}$ & Ridge & $\begin{array}{l}\text { Submesic } \\
\text { Slope } \\
\end{array}$ & $\begin{array}{l}\text { Mesic } \\
\text { Slope }\end{array}$ & $\begin{array}{l}\text { Xeric } \\
\text { Slope } \\
\end{array}$ & Ridge \\
\hline $\begin{array}{l}\text { Black-capped } \\
\text { chickadee }\end{array}$ & $\begin{array}{c}0.58 \\
(0.15)\end{array}$ & $\begin{array}{l}0.41 \\
(0.14)\end{array}$ & $\begin{array}{c}0.44 \\
(0.17)\end{array}$ & $\begin{array}{c}0.59 \\
(0.13)\end{array}$ & $\begin{array}{c}0.62 \\
(0.22)\end{array}$ & $\begin{array}{c}0.59 \\
(0.13)\end{array}$ & $\begin{array}{c}0.22 \\
(0.10)\end{array}$ & $\begin{array}{c}0.44 \\
(0.11)\end{array}$ & $\begin{array}{c}0.60 \\
(0.14)\end{array}$ & $\begin{array}{c}0.24 \\
(0.09)\end{array}$ & $\begin{array}{c}0.41 \\
(0.15)\end{array}$ & $\begin{array}{c}0.38 \\
(0.08)\end{array}$ \\
\hline $\begin{array}{l}\text { Black-throated } \\
\text { blue warbler }\end{array}$ & $\begin{array}{c}0.85 \\
(0.17)\end{array}$ & $\begin{array}{c}0.76 \\
(0.17)\end{array}$ & $\begin{array}{c}0.78 \\
(0.24)\end{array}$ & $\begin{array}{c}0.62 \\
(0.14)\end{array}$ & $\begin{array}{c}0.42 \\
(0.13)\end{array}$ & $\begin{array}{c}0.66 \\
(0.15)\end{array}$ & $\begin{array}{c}0.39 \\
(0.14)\end{array}$ & $\begin{array}{c}0.59 \\
(0.11)\end{array}$ & $\begin{array}{c}0.56 \\
(0.14)\end{array}$ & $\begin{array}{c}0.76 \\
(0.15)\end{array}$ & $\begin{array}{c}0.59 \\
(0.21)\end{array}$ & $\begin{array}{c}0.64 \\
(0.11)\end{array}$ \\
\hline $\begin{array}{l}\text { Black-throated } \\
\text { green warbler }\end{array}$ & $\begin{array}{l}1.85 \\
(0.22)\end{array}$ & $\begin{array}{c}1.17 \\
(0.17)\end{array}$ & $\begin{array}{c}0.78 \\
(0.22)\end{array}$ & $\begin{array}{c}1.62 \\
(0.18)\end{array}$ & $\begin{array}{c}1.54 \\
(0.19)\end{array}$ & $\begin{array}{c}1.41 \\
(0.19)\end{array}$ & $\begin{array}{c}1.22 \\
(0.17)\end{array}$ & $\begin{array}{c}1.74 \\
(0.16)\end{array}$ & $\begin{array}{c}1.64 \\
(0.21)\end{array}$ & $\begin{array}{l}1.76 \\
(0.15)\end{array}$ & $\begin{array}{l}1.35 \\
(0.23)\end{array}$ & $\begin{array}{c}1.97 \\
(0.14)\end{array}$ \\
\hline Blue jay & $\begin{array}{c}0.92 \\
(0.17)\end{array}$ & $\begin{array}{c}0.69 \\
(0.21)\end{array}$ & $\begin{array}{c}0.61 \\
(0.23)\end{array}$ & $\begin{array}{c}0.59 \\
(0.12)\end{array}$ & $\begin{array}{c}0.62 \\
(0.12)\end{array}$ & $\begin{array}{c}0.41 \\
(0.13)\end{array}$ & $\begin{array}{c}0.39 \\
(0.12)\end{array}$ & $\begin{array}{c}0.64 \\
(0.13)\end{array}$ & $\begin{array}{c}0.40 \\
(0.13)\end{array}$ & $\begin{array}{c}0.41 \\
(0.12)\end{array}$ & $\begin{array}{c}0.12 \\
(0.08)\end{array}$ & $\begin{array}{c}0.41 \\
(0.10)\end{array}$ \\
\hline $\begin{array}{l}\text { Blue-headed } \\
\text { vireo }\end{array}$ & $\begin{array}{c}2.04 \\
(0.26)\end{array}$ & $\begin{array}{l}2.07 \\
(0.26)\end{array}$ & $\begin{array}{c}1.39 \\
(0.24)\end{array}$ & $\begin{array}{c}2.26 \\
(0.22)\end{array}$ & $\begin{array}{l}1.46 \\
(0.20)\end{array}$ & $\begin{array}{c}1.38 \\
(0.18)\end{array}$ & $\begin{array}{c}1.22 \\
(0.17)\end{array}$ & $\begin{array}{c}1.59 \\
(0.16)\end{array}$ & $\begin{array}{c}0.48 \\
(0.15)\end{array}$ & $\begin{array}{c}0.28 \\
(0.08)\end{array}$ & $\begin{array}{c}0.35 \\
(0.15)\end{array}$ & $\begin{array}{c}0.74 \\
(0.13)\end{array}$ \\
\hline Canada warbler & $\begin{array}{l}1.12 \\
(0.26)\end{array}$ & $\begin{array}{l}1.34 \\
(0.20)\end{array}$ & $\begin{array}{l}1.78 \\
(0.34)\end{array}$ & $\begin{array}{c}0.64 \\
(0.14)\end{array}$ & $\begin{array}{l}1.27 \\
(0.20)\end{array}$ & $\begin{array}{c}1.14 \\
(0.20)\end{array}$ & $\begin{array}{l}1.67 \\
(0.30)\end{array}$ & $\begin{array}{c}0.64 \\
(0.15)\end{array}$ & $\begin{array}{l}1.08 \\
(0.24)\end{array}$ & $\begin{array}{c}0.93 \\
(0.20)\end{array}$ & $\begin{array}{c}1.24 \\
(0.22)\end{array}$ & $\begin{array}{c}0.44 \\
(0.13)\end{array}$ \\
\hline Dark-eyed junco & $\begin{array}{c}1.08 \\
(0.18)\end{array}$ & $\begin{array}{c}0.62 \\
(0.12)\end{array}$ & $\begin{array}{c}0.44 \\
(0.12)\end{array}$ & $\begin{array}{c}0.56 \\
(0.15)\end{array}$ & $\begin{array}{c}1.23 \\
(0.19)\end{array}$ & $\begin{array}{c}0.97 \\
(0.20)\end{array}$ & $\begin{array}{c}0.61 \\
(0.16)\end{array}$ & $\begin{array}{c}0.69 \\
(0.14)\end{array}$ & $\begin{array}{c}1.08 \\
(0.15)\end{array}$ & $\begin{array}{c}0.72 \\
(0.14)\end{array}$ & $\begin{array}{c}0.82 \\
(0.21)\end{array}$ & $\begin{array}{c}0.74 \\
(0.12)\end{array}$ \\
\hline Eastern towhee & $\begin{array}{c}0.19 \\
(0.08)\end{array}$ & $\begin{array}{c}0.45 \\
(0.12)\end{array}$ & $\begin{array}{c}0.78 \\
(0.24)\end{array}$ & $\begin{array}{c}0.38 \\
(0.12)\end{array}$ & $\begin{array}{c}0.31 \\
(0.11)\end{array}$ & $\begin{array}{c}0.17 \\
(0.07)\end{array}$ & $\begin{array}{c}0.50 \\
(0.19)\end{array}$ & $\begin{array}{c}0.18 \\
(0.09)\end{array}$ & $\begin{array}{c}0.20 \\
(0.10)\end{array}$ & $\begin{array}{c}0.03 \\
(0.03)\end{array}$ & $\begin{array}{c}0.53 \\
(0.21)\end{array}$ & $\begin{array}{c}0.15 \\
(0.07)\end{array}$ \\
\hline $\begin{array}{l}\text { Magnolia } \\
\text { warbler }\end{array}$ & $\begin{array}{c}0.65 \\
(0.16)\end{array}$ & $\begin{array}{c}0.55 \\
(0.19)\end{array}$ & $\begin{array}{c}0.89 \\
(0.28)\end{array}$ & $\begin{array}{c}0.77 \\
(0.15)\end{array}$ & $\begin{array}{c}0.38 \\
(0.14)\end{array}$ & $\begin{array}{c}0.17 \\
(0.09)\end{array}$ & $\begin{array}{c}0.33 \\
(0.16)\end{array}$ & $\begin{array}{c}0.33 \\
(0.10)\end{array}$ & $\begin{array}{c}1.00 \\
(0.22)\end{array}$ & $\begin{array}{c}0.55 \\
(0.17)\end{array}$ & $\begin{array}{c}0.94 \\
(0.23)\end{array}$ & $\begin{array}{c}0.64 \\
(0.12)\end{array}$ \\
\hline Ovenbird & $\begin{array}{c}0.19 \\
(0.08)\end{array}$ & $\begin{array}{c}0.45 \\
(0.13)\end{array}$ & $\begin{array}{c}0.39 \\
(0.20)\end{array}$ & $\begin{array}{c}0.97 \\
(0.13)\end{array}$ & $\begin{array}{c}0.19 \\
(0.10)\end{array}$ & $\begin{array}{c}0.38 \\
(0.12)\end{array}$ & $\begin{array}{c}0.44 \\
(0.17)\end{array}$ & $\begin{array}{c}0.77 \\
(0.13)\end{array}$ & $\begin{array}{c}0.20 \\
(0.10)\end{array}$ & $\begin{array}{c}0.24 \\
(0.11)\end{array}$ & $\begin{array}{c}0.24 \\
(0.11)\end{array}$ & $\begin{array}{c}0.85 \\
(0.11)\end{array}$ \\
\hline Red-eyed vireo & $\begin{array}{c}2.08 \\
(0.25)\end{array}$ & $\begin{array}{c}1.86 \\
(0.20)\end{array}$ & $\begin{array}{c}1.94 \\
(0.29)\end{array}$ & $\begin{array}{c}1.87 \\
(0.20)\end{array}$ & $\begin{array}{c}1.69 \\
(0.21)\end{array}$ & $\begin{array}{c}1.86 \\
(0.18)\end{array}$ & $\begin{array}{c}1.67 \\
(0.23)\end{array}$ & $\begin{array}{c}1.64 \\
(0.17)\end{array}$ & $\begin{array}{c}2.88 \\
(0.16)\end{array}$ & $\begin{array}{c}2.97 \\
(0.13)\end{array}$ & $\begin{array}{c}2.41 \\
(0.24)\end{array}$ & $\begin{array}{c}2.64 \\
(0.17)\end{array}$ \\
\hline
\end{tabular}


Table 1.9. Continued.

\begin{tabular}{|c|c|c|c|c|c|c|c|c|c|c|c|c|}
\hline \multirow[b]{3}{*}{ Species/Guild } & \multicolumn{4}{|c|}{1996} & \multicolumn{4}{|c|}{1997} & \multicolumn{4}{|c|}{1998} \\
\hline & \multicolumn{4}{|c|}{$\begin{array}{c}\text { Mean } \\
(\mathrm{SE})\end{array}$} & \multicolumn{4}{|c|}{$\begin{array}{c}\text { Mean } \\
(\mathrm{SE})\end{array}$} & \multicolumn{4}{|c|}{$\begin{array}{c}\text { Mean } \\
(\mathrm{SE})\end{array}$} \\
\hline & $\begin{array}{c}\text { Submesic } \\
\text { Slope }\end{array}$ & $\begin{array}{l}\text { Mesic } \\
\text { Slope }\end{array}$ & $\begin{array}{l}\text { Xeric } \\
\text { Slope }\end{array}$ & Ridge & $\begin{array}{c}\text { Submesic } \\
\text { Slope }\end{array}$ & $\begin{array}{l}\text { Mesic } \\
\text { Slope }\end{array}$ & $\begin{array}{l}\text { Xeric } \\
\text { Slope }\end{array}$ & Ridge & $\begin{array}{c}\text { Submesic } \\
\text { Slope }\end{array}$ & $\begin{array}{l}\text { Mesic } \\
\text { Slope }\end{array}$ & $\begin{array}{l}\text { Xeric } \\
\text { Slope }\end{array}$ & Ridge \\
\hline \multirow{2}{*}{$\begin{array}{l}\text { Rose-breasted } \\
\text { grosbeak }\end{array}$} & $0 \Omega 8$ & & 030 & 054 & 031 & 034 & 030 & 015 & 032 & & & 010 \\
\hline & $\begin{array}{c}0.08 \\
(0.05)\end{array}$ & $\begin{array}{c}0.41 \\
(0.12)\end{array}$ & $\begin{array}{c}0.39 \\
(0.14)\end{array}$ & $\begin{array}{c}0.54 \\
(0.11)\end{array}$ & $\begin{array}{c}0.31 \\
(0.09)\end{array}$ & $\begin{array}{c}0.34 \\
(0.09)\end{array}$ & $(0.16)$ & $\begin{array}{c}0.15 \\
(0.07)\end{array}$ & $\begin{array}{c}0.32 \\
(0.10)\end{array}$ & $\begin{array}{c}0.10 \\
(0.06)\end{array}$ & $\begin{array}{c}0.00 \\
(0.60)\end{array}$ & $\begin{array}{l}0.10 \\
(0.05)\end{array}$ \\
\hline \multirow[t]{2}{*}{ Scarlet tanager } & 0.58 & 0.69 & 0.50 & 0.79 & 0.54 & 0.48 & 0.44 & 0.41 & 0.80 & 0.66 & 0.82 & 0.62 \\
\hline & $(0.14)$ & $(0.10)$ & $(0.15)$ & $(0.15)$ & $(0.10)$ & $(0.09)$ & $(0.12)$ & $(0.08)$ & $(0.13)$ & $(0.09)$ & $(0.10)$ & $(0.08)$ \\
\hline \multirow[t]{2}{*}{ Veery } & 1.69 & 0.83 & 1.78 & 1.10 & 1.00 & 0.76 & 1.00 & 1.05 & 0.92 & 0.62 & 1.47 & 0.90 \\
\hline & $(0.17)$ & $(0.13)$ & $(0.19)$ & $(0.17)$ & $(0.15)$ & $(0.15)$ & $(0.14)$ & $(0.14)$ & $(0.18)$ & $(0.14)$ & $(0.24)$ & $(0.13)$ \\
\hline \multirow[t]{2}{*}{ Winter wren } & 0.58 & 0.52 & 0.39 & 0.31 & 0.54 & 0.45 & 0.78 & 0.23 & 0.68 & 0.55 & 0.41 & 0.26 \\
\hline & $(0.14)$ & $(0.12)$ & $(0.12)$ & $(0.08)$ & $(0.11)$ & $(0.12)$ & $(0.10)$ & $(0.07)$ & $(0.11)$ & $(0.12)$ & $(0.12)$ & $(0.07)$ \\
\hline
\end{tabular}


Table 1.10. ANOVA and Scheffe' multiple comparison test results comparing songbird abundance (species at $\geq 20 \%$ of point counts) among landtypes on the Westvaco Wildlife and Ecosystem Research Forest, Randolph County, West Virginia, 1997-1998 (unharvested point counts only). Landtypes with the same letter do not differ at the family alpha level of 0.10 . "A" indicates highest abundance, followed by "B", "C", and "D".

\begin{tabular}{|c|c|c|c|c|c|c|}
\hline \multirow[b]{2}{*}{ Species/Guild } & \multirow[b]{2}{*}{$\mathrm{F}$} & \multirow[b]{2}{*}{$\begin{array}{c}\mathrm{P}- \\
\text { value }\end{array}$} & \multicolumn{4}{|c|}{ Landtype } \\
\hline & & & $\begin{array}{l}\text { Submesic } \\
\text { Slope }\end{array}$ & $\begin{array}{l}\text { Mesic } \\
\text { Slope }\end{array}$ & $\begin{array}{l}\text { Xeric } \\
\text { Slope }\end{array}$ & Ridge \\
\hline Total & 1.77 & 0.15 & & & & \\
\hline \multicolumn{7}{|l|}{ Habitat Groups: } \\
\hline Interior & 2.23 & 0.08 & $\mathrm{AB}$ & B & $\mathrm{AB}$ & A \\
\hline Interior-edge & 1.89 & 0.13 & & & & \\
\hline Edge & 2.98 & 0.03 & $\mathrm{AB}$ & A & $\mathrm{AB}$ & $\mathrm{B}$ \\
\hline \multicolumn{7}{|l|}{ Nesting Guilds: } \\
\hline Ground & 4.16 & $<0.01$ & A & $\mathrm{AC}$ & $\mathrm{AB}$ & A \\
\hline Shrub & 0.42 & 0.74 & & & & \\
\hline Subcanopy & 1.00 & 0.39 & & & & \\
\hline Canopy & 7.82 & $<0.01$ & A & A & B & A \\
\hline Cavity & 1.14 & 0.33 & & & & \\
\hline \multicolumn{7}{|l|}{ Edge Species: } \\
\hline American crow & 2.99 & 0.03 & A & $\mathrm{AB}$ & $\mathrm{AB}$ & $\mathrm{B}$ \\
\hline \multicolumn{7}{|l|}{ Interior-edge Species: } \\
\hline Black-capped chickadee & 1.30 & 0.27 & & & & \\
\hline Blue jay & 1.78 & 0.15 & & & & \\
\hline Dark-eyed junco & 6.20 & $<0.01$ & A & $\mathrm{AB}$ & B & B \\
\hline Eastern towhee & 5.08 & $<0.01$ & B & $\mathrm{B}$ & A & $\mathrm{B}$ \\
\hline Red-eyed vireo & 0.76 & 0.52 & & & & \\
\hline \multirow[t]{6}{*}{ Rose-breasted grosbeak } & 1996: & & & & & \\
\hline & 3.32 & 0.02 & $\mathrm{~B}$ & $\mathrm{AB}$ & $\mathrm{AB}$ & A \\
\hline & 1997: & & & & & \\
\hline & 1.28 & 0.28 & & & & \\
\hline & 1998: & & & & & \\
\hline & 2.82 & 0.04 & A & B & B & B \\
\hline \multicolumn{7}{|l|}{ Interior Species: } \\
\hline $\begin{array}{l}\text { Black-throated blue } \\
\text { warbler }\end{array}$ & 0.54 & 0.65 & & & & \\
\hline $\begin{array}{l}\text { Black-throated green } \\
\text { warbler }\end{array}$ & 5.87 & $<0.01$ & A & $A B$ & B & A \\
\hline Blue-headed vireo & 3.51 & 0.02 & $\mathrm{AB}$ & $\mathrm{AB}$ & B & A \\
\hline Canada warbler & 13.75 & $<0.01$ & $\mathrm{~A}$ & $\mathrm{~A}$ & A & B \\
\hline Magnolia warbler & 2.17 & 0.12 & & & & \\
\hline Ovenbird & 20.79 & $<0.01$ & $\mathrm{~B}$ & B & $\mathrm{B}$ & A \\
\hline Scarlet tanager & 0.09 & 0.96 & & & & \\
\hline Veery & 7.94 & $<0.01$ & $\mathrm{ACD}$ & $\mathrm{B}$ & $\mathrm{AC}$ & $\mathrm{BD}$ \\
\hline Winter wren & 6.99 & $<0.01$ & $\mathrm{~A}$ & A & $\mathrm{A}$ & $\mathrm{B}$ \\
\hline
\end{tabular}


Table 1.11. Means and standard errors for total abundance, abundance of habitat groups and nesting guilds, and abundance of individual species (at $\geq 20 \%$ of point counts) by mature mixed woods (1996: $n=48 ; 1997: n=33 ; 98: n=30$ ), mature hardwoods (1996: $n=96 ; 1997: n=84 ; 1998: n=80)$, diameter-limit harvests $(1997: n=17 ; 1998: n=22)$, and two-age harvests $(1997-98: n=10)$ on the Westvaco Wildlife and Ecosystem Research Forest, Randolph County, West Virginia. Means are for untransformed data.

\begin{tabular}{|c|c|c|c|c|c|c|c|c|c|c|}
\hline \multirow[b]{3}{*}{ Guild/Species } & \multirow{2}{*}{\multicolumn{2}{|c|}{$\begin{array}{c}1996 \\
\text { Mean } \\
(\mathrm{SE}) \\
\end{array}$}} & \multirow{2}{*}{\multicolumn{4}{|c|}{$\begin{array}{c}\frac{1997}{\text { Mean }} \\
(\mathrm{SE})\end{array}$}} & \multirow{2}{*}{\multicolumn{4}{|c|}{$\begin{array}{c}\frac{1998}{\text { Mean }} \\
(\mathrm{SE})\end{array}$}} \\
\hline & & & & & & & & & & \\
\hline & $\begin{array}{c}\text { Mature } \\
\text { Mixed wood }\end{array}$ & $\begin{array}{c}\text { Mature } \\
\text { Hardwood }\end{array}$ & $\begin{array}{c}\text { Mature } \\
\text { Mixed wood }\end{array}$ & $\begin{array}{c}\text { Mature } \\
\text { Hardwood }\end{array}$ & $\begin{array}{l}\text { Diameter- } \\
\text { limit }\end{array}$ & $\begin{array}{l}\text { Two- } \\
\text { age }\end{array}$ & $\begin{array}{c}\text { Mature } \\
\text { Mixed wood }\end{array}$ & $\begin{array}{c}\text { Mature } \\
\text { Hardwood }\end{array}$ & $\begin{array}{l}\text { Diameter- } \\
\text { limit }\end{array}$ & $\begin{array}{l}\text { Two- } \\
\text { age }\end{array}$ \\
\hline Total $^{\text {bc }}$ & $\begin{array}{l}12.17 \\
(0.57)\end{array}$ & $\begin{array}{l}13.65 \\
(0.38)\end{array}$ & $\begin{array}{l}10.82 \\
(0.62)\end{array}$ & $\begin{array}{l}11.20 \\
(0.37)\end{array}$ & $\begin{array}{l}13.76 \\
(1.12)\end{array}$ & $\begin{array}{l}14.80 \\
(1.02)\end{array}$ & $\begin{array}{l}10.90 \\
(0.55)\end{array}$ & $\begin{array}{l}11.51 \\
(0.34)\end{array}$ & $\begin{array}{l}13.73 \\
(0.77)\end{array}$ & $\begin{array}{l}16.10 \\
(1.26)\end{array}$ \\
\hline \multicolumn{11}{|l|}{ Habitat Groups: } \\
\hline Interior & $\begin{array}{c}7.83 \\
(0.43)\end{array}$ & $\begin{array}{c}8.76 \\
(0.32)\end{array}$ & $\begin{array}{c}7.30 \\
(0.46)\end{array}$ & $\begin{array}{c}7.21 \\
(0.29)\end{array}$ & $\begin{array}{c}8.59 \\
(0.61)\end{array}$ & $\begin{array}{c}7.40 \\
(0.69)\end{array}$ & $\begin{array}{c}6.83 \\
(0.54)\end{array}$ & $\begin{array}{c}6.85 \\
(0.28)\end{array}$ & $\begin{array}{c}7.82 \\
(0.56)\end{array}$ & $\begin{array}{c}5.60 \\
(0.70)\end{array}$ \\
\hline Interior-edge $\mathrm{e}^{\mathrm{bc}}$ & $\begin{array}{l}4.26 \\
(0.35)\end{array}$ & $\begin{array}{c}4.50 \\
(0.20)\end{array}$ & $\begin{array}{c}3.82 \\
(0.32)\end{array}$ & $\begin{array}{l}3.76 \\
(0.20)\end{array}$ & $\begin{array}{l}5.35 \\
(0.65)\end{array}$ & $\begin{array}{c}6.80 \\
(0.81)\end{array}$ & $\begin{array}{l}4.40 \\
(0.25)\end{array}$ & $\begin{array}{l}4.70 \\
(0.17)\end{array}$ & $\begin{array}{c}5.77 \\
(0.39)\end{array}$ & $\begin{array}{c}7.30 \\
(0.63)\end{array}$ \\
\hline Edge & $\begin{array}{c}1.07 \\
(0.17)\end{array}$ & $\begin{array}{c}1.54 \\
(0.17)\end{array}$ & $\begin{array}{c}0.76 \\
(0.15)\end{array}$ & $\begin{array}{l}1.04 \\
(0.14)\end{array}$ & $\begin{array}{c}0.76 \\
(0.18)\end{array}$ & $\begin{array}{c}2.20 \\
(0.59)\end{array}$ & $\begin{array}{c}0.50 \\
(0.15)\end{array}$ & $\begin{array}{c}0.86 \\
(0.14)\end{array}$ & $\begin{array}{c}0.77 \\
(0.29)\end{array}$ & $\begin{array}{c}3.70 \\
(0.52)\end{array}$ \\
\hline \multicolumn{11}{|l|}{ Nesting Guilds: } \\
\hline Ground $^{\mathrm{bc}}$ & $\begin{array}{c}3.83 \\
(0.28)\end{array}$ & $\begin{array}{c}4.22 \\
(0.20)\end{array}$ & $\begin{array}{c}4.24 \\
(0.37)\end{array}$ & $\begin{array}{c}3.71 \\
(0.21)\end{array}$ & $\begin{array}{c}5.29 \\
(0.72)\end{array}$ & $\begin{array}{c}5.50 \\
(0.67)\end{array}$ & $\begin{array}{c}3.53 \\
(0.36)\end{array}$ & $\begin{array}{c}3.34 \\
(0.23)\end{array}$ & $\begin{array}{c}4.95 \\
(0.37)\end{array}$ & $\begin{array}{c}5.00 \\
(0.52)\end{array}$ \\
\hline Shrub & $\begin{array}{c}0.98 \\
(0.15)\end{array}$ & $\begin{array}{c}1.86 \\
(0.19)\end{array}$ & $\begin{array}{c}0.79 \\
(0.18)\end{array}$ & $\begin{array}{l}1.18 \\
(0.12)\end{array}$ & $\begin{array}{c}0.71 \\
(0.19)\end{array}$ & $\begin{array}{c}2.20 \\
(0.44)\end{array}$ & $\begin{array}{c}0.77 \\
(0.20)\end{array}$ & $\begin{array}{l}1.26 \\
(0.11)\end{array}$ & $\begin{array}{c}1.14 \\
(0.28)\end{array}$ & $\begin{array}{c}3.20 \\
(0.51)\end{array}$ \\
\hline Subcanopy & $\begin{array}{c}3.39 \\
(0.32)\end{array}$ & $\begin{array}{c}3.63 \\
(0.18)\end{array}$ & $\begin{array}{c}2.76 \\
(0.26)\end{array}$ & $\begin{array}{c}2.56 \\
(0.13)\end{array}$ & $\begin{array}{c}2.88 \\
(0.32)\end{array}$ & $\begin{array}{c}2.20 \\
(0.29)\end{array}$ & $\begin{array}{c}4.40 \\
(0.25)\end{array}$ & $\begin{array}{c}3.76 \\
(0.15)\end{array}$ & $\begin{array}{c}3.95 \\
(0.34)\end{array}$ & $\begin{array}{c}4.20 \\
(0.29)\end{array}$ \\
\hline Canopy & $\begin{array}{c}4.63 \\
(0.31)\end{array}$ & $\begin{array}{c}4.95 \\
(0.23)\end{array}$ & $\begin{array}{c}3.58 \\
(0.28)\end{array}$ & $\begin{array}{c}4.01 \\
(0.20)\end{array}$ & $\begin{array}{c}4.94 \\
(0.45)\end{array}$ & $\begin{array}{c}4.40 \\
(0.70)\end{array}$ & $\begin{array}{c}3.30 \\
(0.29)\end{array}$ & $\begin{array}{c}3.46 \\
(0.16)\end{array}$ & $\begin{array}{c}3.32 \\
(0.30)\end{array}$ & $\begin{array}{c}3.00 \\
(0.49)\end{array}$ \\
\hline Cavity ${ }^{\mathrm{bc}}$ & $\begin{array}{c}1.13 \\
(0.16)\end{array}$ & $\begin{array}{c}1.08 \\
(0.11)\end{array}$ & $\begin{array}{c}0.94 \\
(0.19)\end{array}$ & $\begin{array}{c}0.96 \\
(0.09)\end{array}$ & $\begin{array}{c}1.59 \\
(0.32)\end{array}$ & $\begin{array}{c}2.10 \\
(0.28)\end{array}$ & $\begin{array}{c}0.47 \\
(0.09)\end{array}$ & $\begin{array}{c}1.05 \\
(0.11)\end{array}$ & $\begin{array}{c}1.45 \\
(0.27)\end{array}$ & $\begin{array}{c}1.40 \\
(0.45)\end{array}$ \\
\hline
\end{tabular}


Table 1.11. Continued.

\begin{tabular}{|c|c|c|c|c|c|c|c|c|c|c|}
\hline \multirow[b]{3}{*}{ Guild/Species } & \multirow{2}{*}{\multicolumn{2}{|c|}{$\begin{array}{c}1996 \\
\text { Mean } \\
(\mathrm{SE})\end{array}$}} & \multirow{2}{*}{\multicolumn{4}{|c|}{$\begin{array}{c}1997 \\
\text { Mean } \\
(\mathrm{SE})\end{array}$}} & \multirow{2}{*}{\multicolumn{4}{|c|}{$\begin{array}{c}1998 \\
\text { Mean } \\
(\mathrm{SE})\end{array}$}} \\
\hline & & & & & & & & & & \\
\hline & $\begin{array}{c}\text { Mature } \\
\text { Mixed wood }\end{array}$ & $\begin{array}{c}\text { Mature } \\
\text { Hardwood }\end{array}$ & $\begin{array}{c}\text { Mature } \\
\text { Mixed wood }\end{array}$ & $\begin{array}{c}\text { Mature } \\
\text { Hardwood }\end{array}$ & $\begin{array}{c}\text { Diameter- } \\
\text { limit }\end{array}$ & $\begin{array}{l}\text { Two- } \\
\text { age }\end{array}$ & $\begin{array}{c}\text { Mature } \\
\text { Mixed wood }\end{array}$ & $\begin{array}{c}\text { Mature } \\
\text { Hardwood }\end{array}$ & $\begin{array}{c}\text { Diameter- } \\
\text { limit }\end{array}$ & $\begin{array}{l}\text { Two- } \\
\text { age }\end{array}$ \\
\hline \multicolumn{11}{|l|}{ Edge Species: } \\
\hline American crow & $\begin{array}{c}0.11 \\
(0.06)\end{array}$ & $\begin{array}{c}0.66 \\
(0.10)\end{array}$ & $\begin{array}{c}0.15 \\
(0.08)\end{array}$ & $\begin{array}{c}0.39 \\
(0.08)\end{array}$ & $\begin{array}{c}0.06 \\
(0.06)\end{array}$ & $\begin{array}{c}0.80 \\
(0.39)\end{array}$ & $\begin{array}{c}0.30 \\
(0.11)\end{array}$ & $\begin{array}{c}0.40 \\
(0.08)\end{array}$ & $\begin{array}{c}0.05 \\
(0.05)\end{array}$ & $\begin{array}{c}0.60 \\
(0.16)\end{array}$ \\
\hline \multicolumn{11}{|l|}{ Interior-edge } \\
\hline \multicolumn{11}{|l|}{ Species: } \\
\hline $\begin{array}{l}\text { Black-capped } \\
\text { chickadee }\end{array}$ & $\begin{array}{c}0.67 \\
(0.13)\end{array}$ & $\begin{array}{c}0.49 \\
(0.07)\end{array}$ & $\begin{array}{c}0.58 \\
(0.17)\end{array}$ & $\begin{array}{c}0.44 \\
(0.07)\end{array}$ & $\begin{array}{c}0.94 \\
(0.30)\end{array}$ & $\begin{array}{c}1.00 \\
(0.21)\end{array}$ & $\begin{array}{c}0.30 \\
(0.09)\end{array}$ & $\begin{array}{c}0.43 \\
(0.07)\end{array}$ & $\begin{array}{c}1.00 \\
(0.20)\end{array}$ & $\begin{array}{c}0.60 \\
(0.22)\end{array}$ \\
\hline Blue jay & $\begin{array}{c}1.09 \\
(0.19)\end{array}$ & $\begin{array}{c}0.61 \\
(0.08)\end{array}$ & $\begin{array}{c}0.64 \\
(0.14)\end{array}$ & $\begin{array}{c}0.51 \\
(0.07)\end{array}$ & $\begin{array}{c}0.65 \\
(0.15)\end{array}$ & $\begin{array}{c}0.70 \\
(0.21)\end{array}$ & $\begin{array}{c}0.27 \\
(0.10)\end{array}$ & $\begin{array}{c}0.40 \\
(0.07)\end{array}$ & $\begin{array}{c}0.45 \\
(0.17)\end{array}$ & $\begin{array}{r}0.30 \\
(0.15)\end{array}$ \\
\hline $\begin{array}{l}\text { Dark-eyed } \\
\text { junco abc }\end{array}$ & $\begin{array}{c}1.04 \\
(0.15)\end{array}$ & $\begin{array}{c}0.65 \\
(0.08)\end{array}$ & $\begin{array}{c}1.15 \\
(0.20)\end{array}$ & $\begin{array}{c}0.83 \\
(0.10)\end{array}$ & $\begin{array}{c}1.88 \\
(0.35)\end{array}$ & $\begin{array}{c}1.30 \\
(0.33)\end{array}$ & $\begin{array}{c}1.10 \\
(0.15)\end{array}$ & $\begin{array}{c}0.73 \\
(0.08)\end{array}$ & $\begin{array}{c}1.73 \\
(0.22)\end{array}$ & $\begin{array}{c}1.40 \\
(0.34)\end{array}$ \\
\hline Eastern towhee $^{\mathrm{b}}$ & $\begin{array}{c}0.26 \\
(0.08)\end{array}$ & $\begin{array}{c}0.50 \\
(0.08)\end{array}$ & $\begin{array}{c}0.21 \\
(0.08)\end{array}$ & $\begin{array}{c}0.30 \\
(0.07)\end{array}$ & $\begin{array}{c}0.59 \\
(0.21)\end{array}$ & $\begin{array}{l}1.70 \\
(0.33)\end{array}$ & $\begin{array}{l}0.10 \\
(0.06)\end{array}$ & $\begin{array}{c}0.20 \\
(0.05)\end{array}$ & $\begin{array}{c}0.45 \\
(0.13)\end{array}$ & $\begin{array}{l}1.50 \\
(0.27)\end{array}$ \\
\hline Red-eyed Vireo ${ }^{\text {bc }}$ & $\begin{array}{c}1.54 \\
(0.18)\end{array}$ & $\begin{array}{l}2.15 \\
(0.12)\end{array}$ & $\begin{array}{c}1.42 \\
(0.17)\end{array}$ & $\begin{array}{c}1.85 \\
(0.11)\end{array}$ & $\begin{array}{c}1.35 \\
(0.26)\end{array}$ & $\begin{array}{c}1.90 \\
(0.28)\end{array}$ & $\begin{array}{c}2.60 \\
(0.14)\end{array}$ & $\begin{array}{c}2.83 \\
(0.11)\end{array}$ & $\begin{array}{c}2.55 \\
(0.23)\end{array}$ & $\begin{array}{r}2.50 \\
(0.17)\end{array}$ \\
\hline $\begin{array}{l}\text { Rose-breasted } \\
\text { grosbeak }^{\mathrm{b}}\end{array}$ & $\begin{array}{c}0.33 \\
(0.08)\end{array}$ & $\begin{array}{c}0.43 \\
(0.06)\end{array}$ & $\begin{array}{c}0.30 \\
(0.08)\end{array}$ & $\begin{array}{c}0.30 \\
(0.06)\end{array}$ & $\begin{array}{c}0.53 \\
(0.15)\end{array}$ & $\begin{array}{c}0.30 \\
(0.15)\end{array}$ & $\begin{array}{c}0.07 \\
(0.05)\end{array}$ & $\begin{array}{c}0.16 \\
(0.04)\end{array}$ & $\begin{array}{c}0.45 \\
(0.13)\end{array}$ & $\begin{array}{c}0.50 \\
(0.17)\end{array}$ \\
\hline \multicolumn{11}{|l|}{ Interior Species: } \\
\hline $\begin{array}{l}\text { Black-throated } \\
\text { blue warbler }\end{array}$ & $\begin{array}{c}0.35 \\
(0.08)\end{array}$ & $\begin{array}{c}0.82 \\
(0.10)\end{array}$ & $\begin{array}{c}0.39 \\
(0.14)\end{array}$ & $\begin{array}{c}0.56 \\
(0.07)\end{array}$ & $\begin{array}{c}0.47 \\
(0.17)\end{array}$ & $\begin{array}{c}0.50 \\
(0.22)\end{array}$ & $\begin{array}{c}0.47 \\
(0.12)\end{array}$ & $\begin{array}{c}0.71 \\
(0.08)\end{array}$ & $\begin{array}{c}0.59 \\
(0.14)\end{array}$ & $\begin{array}{r}0.20 \\
(0.13)\end{array}$ \\
\hline $\begin{array}{l}\text { Black-throated } \\
\text { green warbler }\end{array}$ & $\begin{array}{c}1.61 \\
(0.18)\end{array}$ & $\begin{array}{c}1.48 \\
(0.11)\end{array}$ & $\begin{array}{c}1.36 \\
(0.18)\end{array}$ & $\begin{array}{c}1.63 \\
(0.10)\end{array}$ & $\begin{array}{c}2.00 \\
(0.31)\end{array}$ & $\begin{array}{c}1.10 \\
(0.23)\end{array}$ & $\begin{array}{c}1.53 \\
(0.16)\end{array}$ & $\begin{array}{c}1.86 \\
(0.10)\end{array}$ & $\begin{array}{c}1.64 \\
(0.19)\end{array}$ & $\begin{array}{c}0.80 \\
(0.25)\end{array}$ \\
\hline
\end{tabular}


Table 1.11. Continued.

\begin{tabular}{|c|c|c|c|c|c|c|c|c|c|c|}
\hline \multirow[b]{3}{*}{ Guild/Species } & \multirow{2}{*}{\multicolumn{2}{|c|}{$\begin{array}{c}1996 \\
\text { Mean } \\
(\mathrm{SE}) \\
\end{array}$}} & \multicolumn{4}{|c|}{1997} & \multicolumn{4}{|c|}{1998} \\
\hline & & & \multicolumn{4}{|c|}{$\begin{array}{c}\text { Mean } \\
(\mathrm{SE}) \\
\end{array}$} & \multicolumn{4}{|c|}{$\begin{array}{c}\text { Mean } \\
(\mathrm{SE})\end{array}$} \\
\hline & $\begin{array}{c}\text { Mature } \\
\text { Mixed wood }\end{array}$ & $\begin{array}{c}\text { Mature } \\
\text { Hardwood }\end{array}$ & $\begin{array}{c}\text { Mature } \\
\text { Mixed wood }\end{array}$ & $\begin{array}{c}\text { Mature } \\
\text { Hardwoods }\end{array}$ & $\begin{array}{l}\text { Diameter- } \\
\text { limit }\end{array}$ & $\begin{array}{l}\text { Two- } \\
\text { age }\end{array}$ & $\begin{array}{c}\text { Mature } \\
\text { Mixed wood }\end{array}$ & $\begin{array}{c}\text { Mature } \\
\text { Hardwood }\end{array}$ & $\begin{array}{l}\text { Diameter- } \\
\text { limit }\end{array}$ & $\begin{array}{l}\text { Two- } \\
\text { age }\end{array}$ \\
\hline \multirow{2}{*}{$\begin{array}{l}\text { Blue-headed } \\
\text { vireo }^{\mathrm{b}}\end{array}$} & 178 & 215 & 164 & 142 & 2006 & 220 & 050 & 050 & 001 & 030 \\
\hline & $(0.18)$ & $(0.14)$ & $(0.16)$ & $(0.11)$ & $(0.30)$ & $(0.20)$ & $(0.13)$ & $(0.08)$ & $(0.17)$ & $(0.21)$ \\
\hline \multirow[t]{2}{*}{ Canada warbler ${ }^{\mathrm{a}}$} & 1.04 & 1.01 & 1.52 & 0.93 & 1.53 & 1.50 & 1.03 & 0.73 & 1.14 & 1.20 \\
\hline & $(0.20)$ & $(0.11)$ & $(0.20)$ & $(0.12)$ & $(0.24)$ & $(0.34)$ & $(0.20)$ & $(0.11)$ & $(0.23)$ & $(0.25)$ \\
\hline \multicolumn{11}{|l|}{ Magnolia } \\
\hline \multirow[t]{2}{*}{ warbler } & 0.98 & 0.73 & 0.45 & 0.25 & 1.00 & 0.00 & 1.27 & 0.54 & 1.09 & 0.10 \\
\hline & $(0.15)$ & $(0.10)$ & $(0.12)$ & $(0.06)$ & $(0.17)$ & $(0.00)$ & $(0.20)$ & $(0.09)$ & $(0.19)$ & $(0.10)$ \\
\hline \multirow[t]{2}{*}{ Ovenbird } & 0.15 & 0.69 & 0.09 & 0.60 & 0.00 & 0.40 & 0.00 & 0.63 & 0.14 & 0.00 \\
\hline & $(0.05)$ & $(0.08)$ & $(0.05)$ & $(0.08)$ & $(0.00)$ & $(0.27)$ & $(0.00)$ & $(0.08)$ & $(0.07)$ & $(0.00)$ \\
\hline \multirow[t]{2}{*}{ Scarlet tanager } & 0.52 & 0.70 & 0.39 & 0.46 & 0.47 & 0.90 & 0.80 & 0.65 & 0.41 & 1.30 \\
\hline & $(0.09)$ & $(0.08)$ & $(0.09)$ & $(0.05)$ & $(0.12)$ & $(0.18)$ & $(0.09)$ & $(0.06)$ & $(0.13)$ & $(0.30)$ \\
\hline \multirow[t]{2}{*}{ Veery $^{\mathrm{a}}$} & 1.24 & 1.33 & 0.85 & 0.96 & 0.71 & 0.90 & 0.90 & 0.93 & 0.86 & 0.80 \\
\hline & $(0.13)$ & $(0.11)$ & $(0.15)$ & $(0.09)$ & $(0.19)$ & $(0.23)$ & $(0.17)$ & $(0.10)$ & $(0.14)$ & $(0.13)$ \\
\hline \multirow[t]{2}{*}{ Winter wren $^{\mathrm{a}}$} & 0.52 & 0.42 & 0.76 & 0.32 & 0.82 & 0.60 & 0.80 & 0.33 & 0.77 & 0.40 \\
\hline & $(0.08)$ & $(0.06)$ & $(0.10)$ & $(0.05)$ & $(0.18)$ & $(0.16)$ & $(0.11)$ & $(0.05)$ & $(0.11)$ & $(0.16)$ \\
\hline
\end{tabular}

${ }^{\text {a }}$ Species for which there was a significant $(\mathrm{P}<0.05)$ pre-harvest abundance by habitat interaction.

${ }^{b}$ Species with a significant $(\mathrm{P}<0.05)$ correlation between abundance and vertical structure index.

${ }^{c}$ Species with a significant $(\mathrm{P}<0.05)$ abundance by VSI interaction. 
Table 1.12. ANCOVA and Scheffe' multiple comparison test results comparing songbird abundances (species at $\geq 20 \%$ of point counts) among forest habitats on the Westvaco Wildlife and Ecosystem Research Forest, Randolph County, West Virginia, 1997-1998. Habitats with the same letter do not differ at the family alpha level of 0.10. "A" indicates highest abundance, followed by "B", "C", and "D".

\begin{tabular}{|c|c|c|c|c|c|c|}
\hline \multirow[b]{2}{*}{ Group/Species } & \multirow[b]{2}{*}{$\mathrm{F}$} & \multirow[b]{2}{*}{ P-value } & \multicolumn{4}{|c|}{ Habitats } \\
\hline & & & $\begin{array}{c}\text { Mature } \\
\text { Mixed wood }\end{array}$ & $\begin{array}{c}\text { Mature } \\
\text { Hardwood }\end{array}$ & $\begin{array}{c}\text { Diameter- } \\
\text { limit }\end{array}$ & Two-age \\
\hline Total & 8.26 & $<0.01$ & $\mathrm{~B}$ & $\mathrm{~B}$ & $\mathrm{~A}$ & $\mathrm{~A}$ \\
\hline \multicolumn{7}{|l|}{ Habitat Groups: } \\
\hline Interior & 2.18 & 0.09 & $\mathrm{AB}$ & B & $\mathrm{A}$ & $\mathrm{AB}$ \\
\hline Interior-edge & 11.42 & $<0.01$ & $\mathrm{C}$ & $\mathrm{C}$ & $\mathrm{B}$ & $\mathrm{A}$ \\
\hline \multirow[t]{4}{*}{ Edge } & 1997: & & & & & \\
\hline & 1.92 & 0.13 & $\mathrm{~B}$ & B & $\mathrm{B}$ & A \\
\hline & 1998: & & & & & \\
\hline & 13.00 & $<0.01$ & $\mathrm{~B}$ & B & $\mathrm{B}$ & A \\
\hline \multicolumn{7}{|l|}{ Nesting Guilds: } \\
\hline$\overline{\text { Ground }}$ & 7.06 & $<0.01$ & B & B & $\mathrm{A}$ & A \\
\hline Shrub & 8.42 & $<0.01$ & $\mathrm{C}$ & B & $\mathrm{CD}$ & A \\
\hline Subcanopy & 1.97 & 0.12 & & & & \\
\hline Canopy & 1.22 & 0.30 & & & & \\
\hline Cavity & 8.94 & $<0.01$ & B & B & A & A \\
\hline \multicolumn{7}{|l|}{ Edge Species: } \\
\hline American crow & 2.68 & 0.05 & $\mathrm{BC}$ & $\mathrm{AC}$ & $\mathrm{B}$ & A \\
\hline \multicolumn{7}{|l|}{ Interior-edge Species: } \\
\hline $\begin{array}{l}\text { Black-capped } \\
\text { chickadee }\end{array}$ & 6.27 & $<0.01$ & B & B & A & $\mathrm{AB}$ \\
\hline Blue jay & 0.29 & 0.83 & & & & \\
\hline Dark-eyed junco & 11.26 & $<0.01$ & B & B & A & $\mathrm{AB}$ \\
\hline Eastern towhee & 20.89 & $<0.01$ & $\mathrm{C}$ & $\mathrm{BC}$ & $\mathrm{B}$ & $\mathrm{A}$ \\
\hline Red-eyed vireo & 1.74 & 0.16 & & & & \\
\hline Rose-breasted grosbeak & 2.91 & 0.03 & B & B & A & $\mathrm{AB}$ \\
\hline \multicolumn{7}{|l|}{ Black-throated green } \\
\hline Blue-headed vireo & 2.72 & 0.04 & $\mathrm{AB}$ & B & A & $\mathrm{AB}$ \\
\hline Canada warbler & 6.21 & $<0.01$ & A & B & $\mathrm{AB}$ & $\mathrm{AB}$ \\
\hline \multirow[t]{4}{*}{ Magnolia warbler } & 1997: & & & & & \\
\hline & 5.52 & $<0.01$ & $\mathrm{~B}$ & B & A & $\mathrm{B}$ \\
\hline & 1998: & & & & & \\
\hline & 9.82 & $<0.01$ & A & B & $\mathrm{AC}$ & $\mathrm{B}$ \\
\hline Ovenbird & 11.68 & $<0.01$ & B & A & $\mathrm{B}$ & $\mathrm{B}$ \\
\hline Scarlet tanager & 5.53 & $<0.01$ & $\mathrm{~B}$ & B & $\mathrm{B}$ & $\mathrm{A}$ \\
\hline Veery & 0.42 & 0.74 & & & & \\
\hline Winter wren & 15.34 & $<0.01$ & A & $\mathrm{B}$ & A & $\mathrm{AB}$ \\
\hline
\end{tabular}


Table 1.13. Number of nests, exposure days, daily survival, and total survival for songbird nests by treatment found on the Westvaco Wildlife and Ecosystem Research Forest, Randolph County, West Virginia, 1996-1998.

\begin{tabular}{|c|c|c|c|c|c|c|}
\hline \multirow[b]{2}{*}{ Treatment } & \multirow[b]{2}{*}{$\begin{array}{l}\text { No. } \\
\text { Nests }\end{array}$} & \multicolumn{2}{|c|}{ Incubation } & \multicolumn{2}{|c|}{ Brood } & \multirow[b]{2}{*}{$\begin{array}{c}\text { Total } \\
\text { Survival }\end{array}$} \\
\hline & & $\begin{array}{c}\text { Exposure } \\
\text { Days }\end{array}$ & $\begin{array}{c}\text { Daily } \\
\text { Survival }\end{array}$ & $\begin{array}{c}\text { Exposure } \\
\text { Days }\end{array}$ & $\begin{array}{c}\text { Daily } \\
\text { Survival }\end{array}$ & \\
\hline \multicolumn{7}{|l|}{ 1996: } \\
\hline Diameter-Limit No Harvest Control & 13 & 51.5 & 0.961 & 86.0 & 0.953 & 0.372 \\
\hline Diameter-Limit Pre-harvest & 20 & 92.0 & 0.902 & 82.5 & 0.951 & 0.149 \\
\hline Two-age No Harvest Control & 30 & 183.5 & 0.907 & 98.0 & 0.990 & 0.187 \\
\hline Two-age Pre-Harvest & 6 & 30.0 & 0.900 & 31.0 & 1.000 & 0.229 \\
\hline \multicolumn{7}{|l|}{ 1997: } \\
\hline Diameter-Limit No Harvest Control & 16 & 72.5 & 0.903 & 56.0 & 0.946 & 0.142 \\
\hline Diameter-Limit Periphery & 5 & 21.5 & 0.953 & 25.5 & 0.961 & 0.357 \\
\hline Diameter-Limit Harvest & 3 & 21.5 & 1.000 & 26.0 & 0.961 & 0.649 \\
\hline Two-age No Harvest Control & 15 & 102.5 & 0.941 & 77.5 & 0.961 & 0.282 \\
\hline Two-age Periphery & 12 & 97.5 & 0.938 & 39.0 & 0.897 & 0.143 \\
\hline Two-age Harvest & 5 & 35.5 & 0.944 & 20.0 & 0.950 & 0.291 \\
\hline \multicolumn{7}{|l|}{ 1998: } \\
\hline Diameter-Limit No Harvest Control & 10 & 55.0 & 0.964 & 59.5 & 0.933 & 0.284 \\
\hline Diameter-Limit Periphery & 14 & 56.0 & 0.964 & 84.5 & 0.976 & 0.464 \\
\hline Diameter-Limit Harvest & 15 & 57.0 & 1.000 & 89.0 & 0.944 & 0.503 \\
\hline Two-age No Harvest Control & 21 & 109.0 & 0.927 & 106.0 & 0.981 & 0.290 \\
\hline Two-age Periphery & 7 & 34.0 & 0.971 & 48.0 & 0.917 & 0.231 \\
\hline Two-age Harvest & 7 & 14.5 & 1.000 & 43.0 & 0.977 & 0.771 \\
\hline
\end{tabular}


Table 1.14. Means, standard errors (SE), and 95\% confidence intervals (CI) for habitat variables surrounding successful ( $\mathrm{n}=90$ ) and predated nests $(n=95)$ on the Westvaco Wildlife and Ecosystem Research Forest, Randolph County, West Virginia, 1996-1998.

\begin{tabular}{|c|c|c|c|c|c|c|c|c|}
\hline \multirow[b]{3}{*}{ Variable } & \multicolumn{4}{|c|}{ Successful Nests } & \multicolumn{4}{|c|}{ Predated Nests } \\
\hline & \multirow[b]{2}{*}{ Mean } & \multirow[b]{2}{*}{ SE } & \multicolumn{2}{|c|}{$95 \%$ CI } & \multirow[b]{2}{*}{ Mean } & \multirow[b]{2}{*}{ SE } & \multicolumn{2}{|c|}{$95 \% \mathrm{CI}$} \\
\hline & & & Lower & Upper & & & Lower & Upper \\
\hline \multicolumn{9}{|l|}{ Stems: } \\
\hline 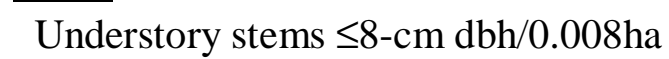 & 120.3 & 22.8 & 75.0 & 165.6 & 139.2 & 21.3 & 97.1 & 181.6 \\
\hline Trees $>8-29.9-\mathrm{cm} \mathrm{dbh} / 0.04 \mathrm{ha}$ & 14.6 & 0.8 & 12.9 & 16.2 & 12.8 & 0.7 & 11.4 & 14.2 \\
\hline Trees $\geq 30.0-\mathrm{cm} \mathrm{dbh} / 0.04 \mathrm{ha}$ & 5.09 & 0.3 & 4.45 & 5.7 & 4.9 & 0.3 & 4.3 & 5.4 \\
\hline \multicolumn{9}{|l|}{ Canopy Cover (percent): } \\
\hline$>0.5-3 \mathrm{~m}$ & 44.5 & 2.1 & 40.3 & 48.7 & 49.9 & 2.6 & 44.8 & 55.0 \\
\hline$>3-6 m$ & 51.5 & 2.6 & 46.4 & 56.7 & 53.6 & 2.5 & 48.7 & 58.6 \\
\hline$>6-12-\mathrm{m}$ & 48.5 & 2.3 & 43.9 & 53.0 & 47.8 & 2.3 & 43.2 & 52.4 \\
\hline$>12-18-m$ & 45.4 & 2.3 & 40.9 & 49.9 & 45.6 & 2.4 & 40.8 & 50.4 \\
\hline$>18-24-m$ & 48.5 & 3.2 & 42.2 & 54.8 & 48.9 & 2.7 & 43.6 & 54.2 \\
\hline$>24-\mathrm{m}$ & 31.8 & 3.1 & 25.6 & 38.1 & 30.8 & 2.7 & 25.5 & 36.2 \\
\hline \multicolumn{9}{|l|}{ Ground Cover (percent): } \\
\hline Green & 27.6 & 2.2 & 23.2 & 32.0 & 29.4 & 2.2 & 25.1 & 33.7 \\
\hline Litter & 50.7 & 2.3 & 46.0 & 55.4 & 49.5 & 2.1 & 45.3 & 53.6 \\
\hline Wood & 8.7 & 1.0 & 6.7 & 10.8 & 8.9 & 0.9 & 7.2 & 10.7 \\
\hline Bareground & 9.9 & 1.4 & 7.2 & 12.6 & 9.2 & 1.6 & 6.1 & 12.4 \\
\hline Distance to edge (m) & 11.6 & 1.9 & 7.8 & 15.3 & 10.4 & 1.6 & 7.3 & 13.6 \\
\hline
\end{tabular}




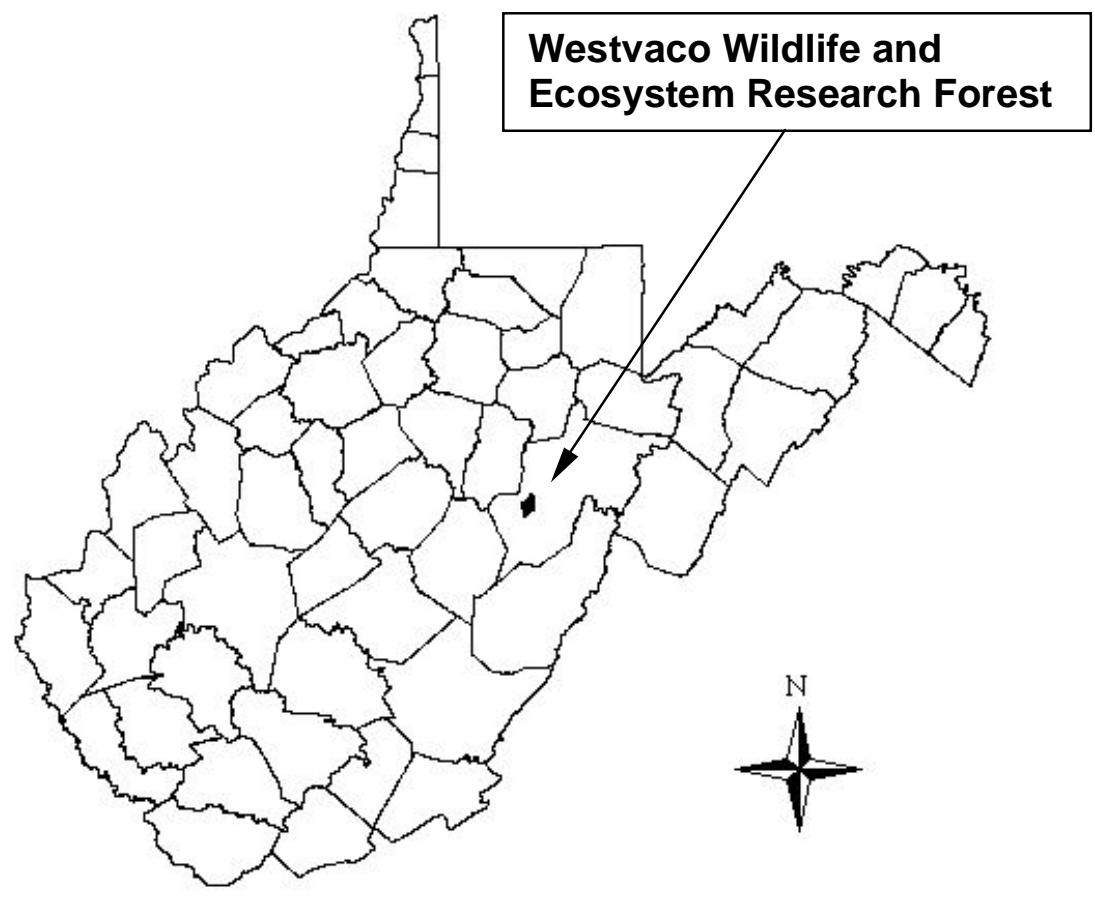

Figure 1.1. Location of the Westvaco Wildife and Ecosystem Research Forest in Randolph County, West Virginia. 

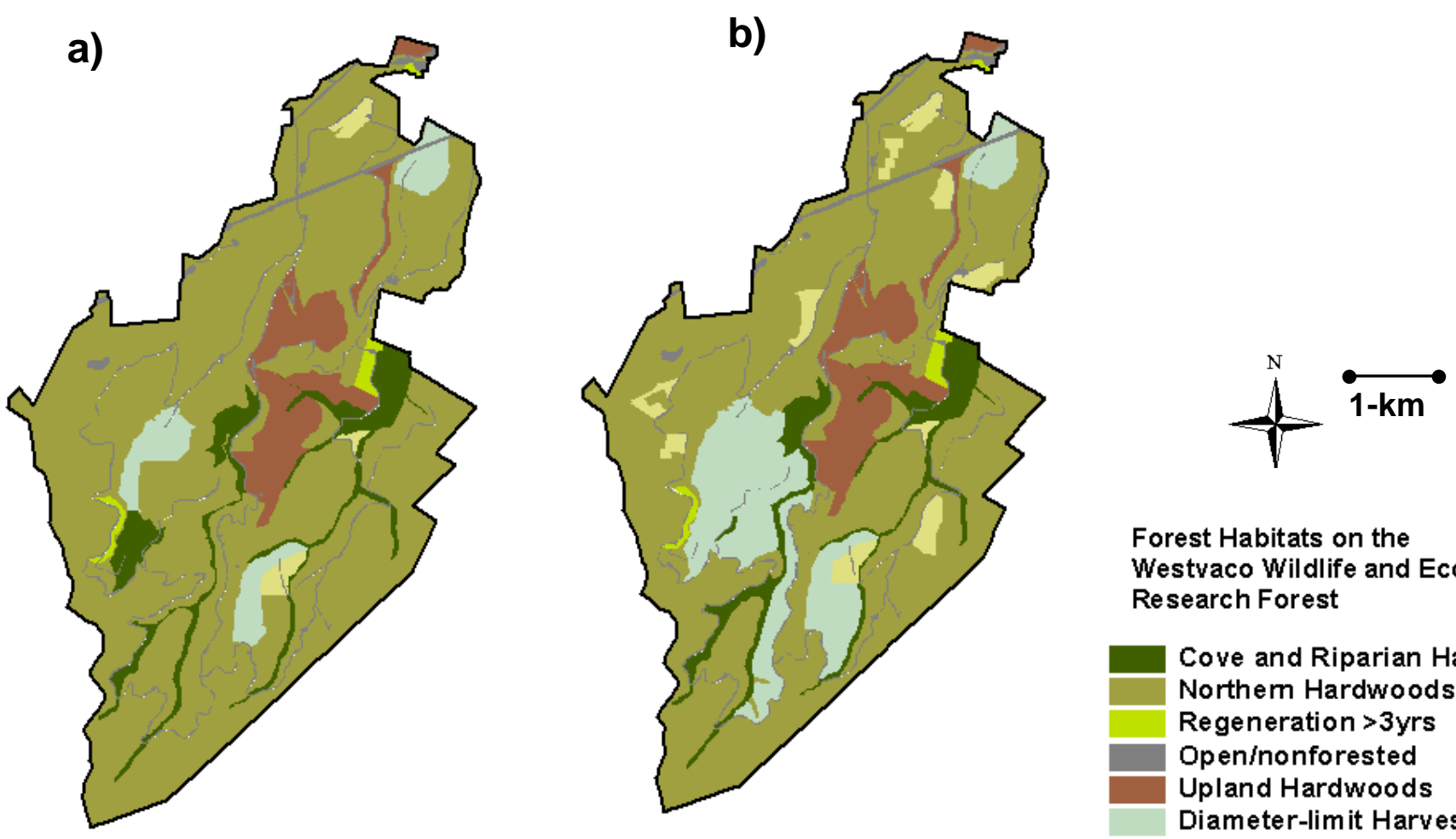

Forest Habitats on the

Westvaco Wildlife and Ecosystem Research Forest

Cove and Riparian Hardwoods

Northem Hardwoods

Regeneration >3yrs

Open/nonforested

Upland Hardwoods

Diameter-lim it Harvest

Regeneration Harvest

Figure 1.2. Forest types on the Westvaco Wildlife and Ecosystem Research Forest, Randolph County, West Virginia in a) 1996 and b) 1998. 


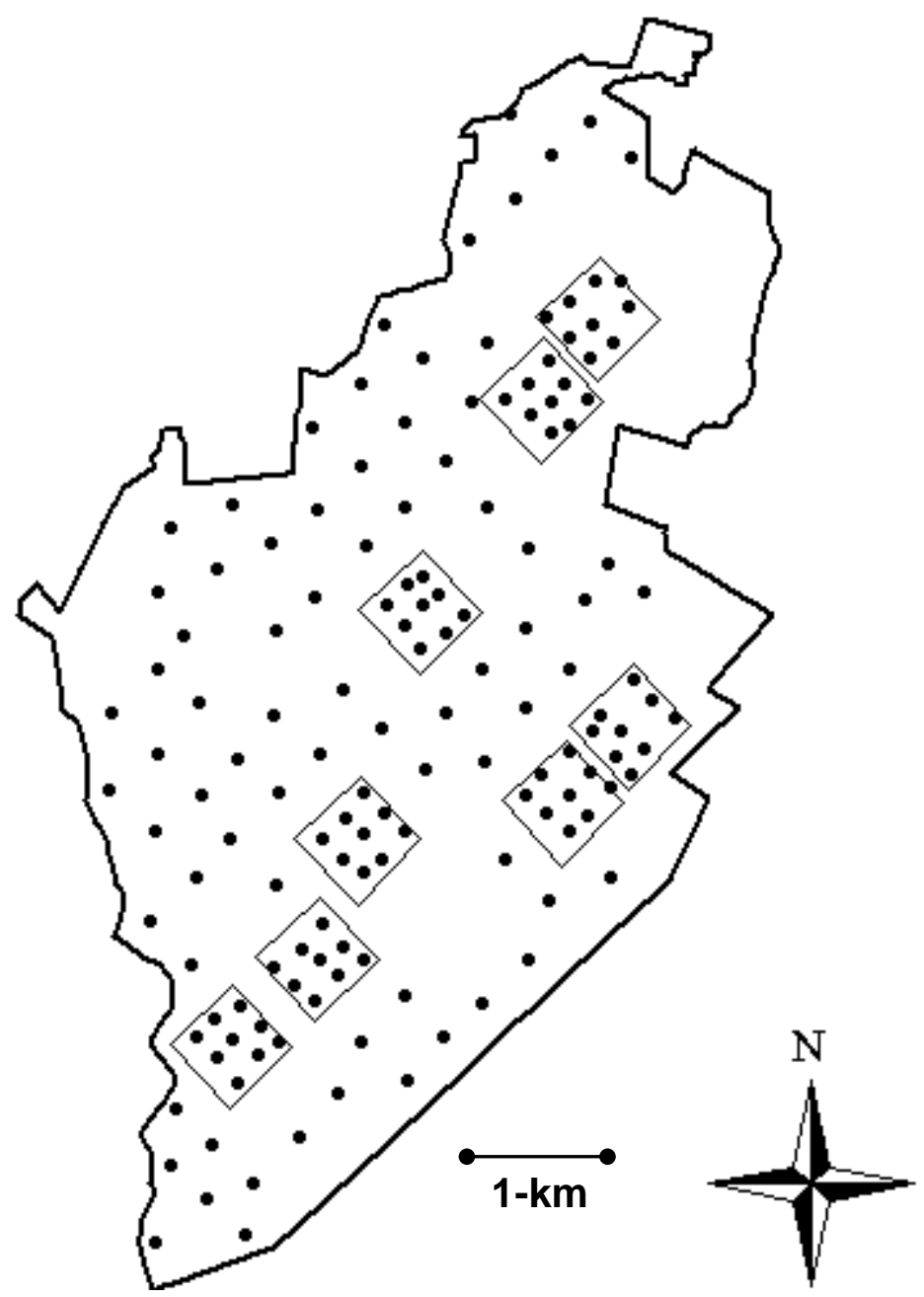

Figure 1.3. Location of nest plots and point count plots on the Westvaco Wildlife and Ecosystem Research Forest, Randolph County, West Virginia, 1996-1998. 


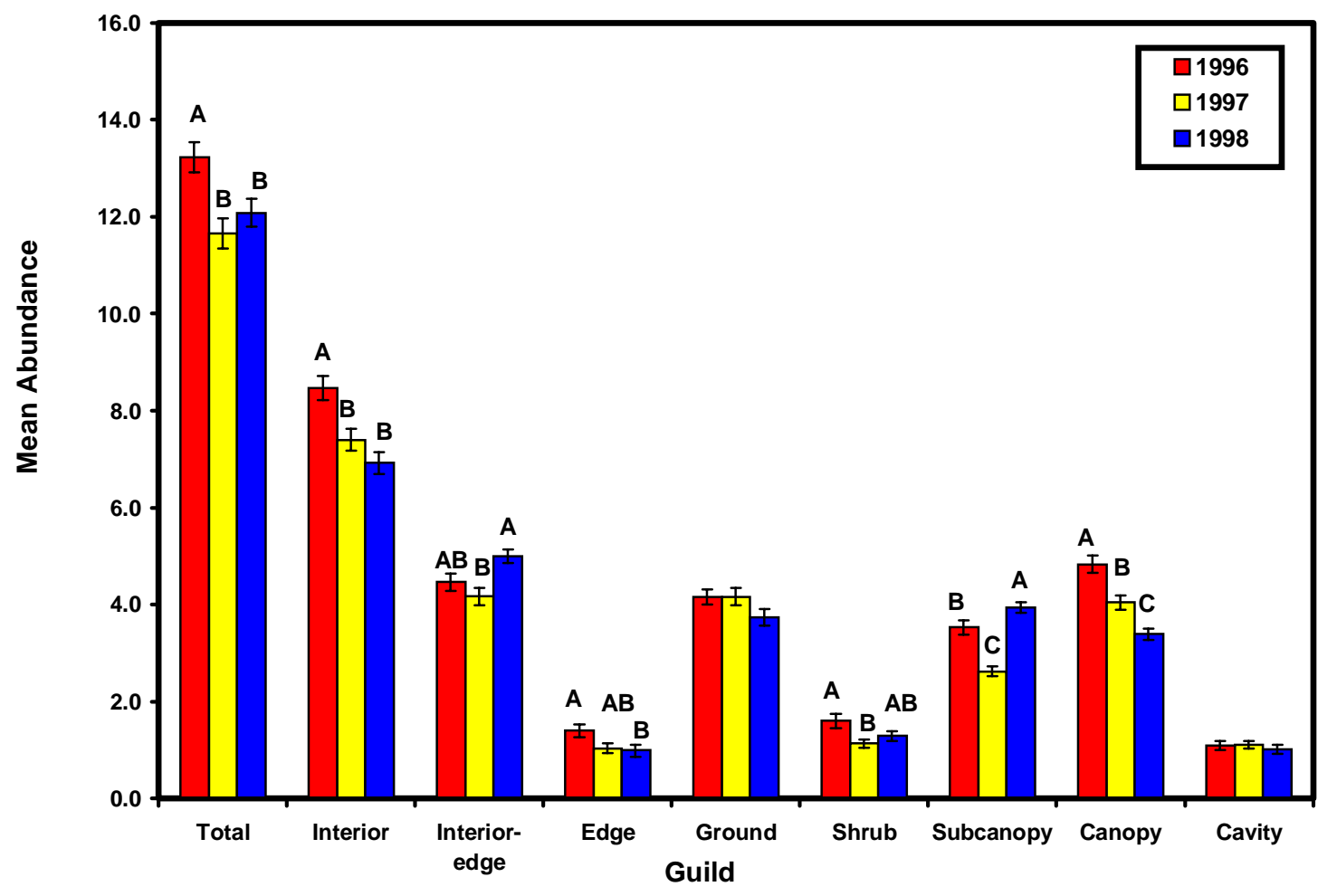

Figure 1.4. Mean abundance of total songbirds, habitat groups, and nesting guilds on the Westvaco Wildlife and Ecosystem Research Forest, Randolph County, West Virginia, 1996-1998. Bars within each group/guild with the same letter do not differ (Scheffe' Multiple Comparison Test, family $\mathrm{P} \leq \mathbf{0 . 1 0}$ ). Means are for untransformed data. 


\section{CHAPTER 2}

RESPONSES OF SONGBIRDS TO DIAMETER-LIMIT CUTTING IN THE CENTRAL APPALACHIANS OF WEST VIRGINIA 


\begin{abstract}
Diameter-limit harvesting, as opposed to silviculturally-accepted systems such as clearcutting, single-tree selection, and two-age harvesting, is a commonly used forest harvesting technique among West Virginia landowners. Despite its widespread use, little is known about the effects of diameter-limit harvesting on songbird abundance and reproductive success. I evaluated the effects of this technique on songbird populations on the Westvaco Wildlife and Ecosystem Research Forest (WWERF) in the Allegheny Mountains of West Virginia. I examined songbird abundances throughout the forest and nesting success on four 40-ha plots in 1996. In 1997, two plots were harvested using the diameter-limit technique where most trees $\geq 45$.7-cm were removed. Abundance and nesting success again were monitored in 1997 and 1998. Results indicate that the abundances of most songbird species present prior to harvest changed little after timber removal. Two species, the Canada warbler (Wilsonia canadensis) and Dark-eyed junco (Junco hyemalis), were more abundant in harvested areas than unharvested forest. One habitat group, interior-edge species, was more abundant in harvested areas. Total abundance of songbirds also was higher in harvested areas. Overall nest survival in harvested areas was higher both 1- (37.7\%) and 2-years (46.4\%) after harvesting than before harvesting (14.9\%). Only large-diameter trees were removed on the WWERF, and intermediate and subcanopy trees remained standing, retaining considerable structural heterogenity. Stands that were harvested differed from unharvested stands by having more snags, higher density of trees $\geq 8-14.9-\mathrm{cm}$, and a higher percent cover of down wood. Canopy cover over 24-m, density of saplings, and the amount of leaf litter decreased after harvesting, whereas canopy cover $\geq 0.5-3-\mathrm{m}$ and the percent of bareground increased. Diameter-limit harvesting as practiced in my study does not appear to adversely affect forest-dwelling songbirds in the central Appalachians in the short-term.
\end{abstract}

\title{
1. Introduction:
}

Generally, research examining songbird response to partial harvesting falls into two categories: uneven-aged (single-tree selection and group selection) where a high amount of basal area is retained, and even-aged (two-age or deferment harvests), where much less residual basal area is retained and often removed some years following initial harvest. Songbird communities respond differently to these types of silvicultural systems. Small changes in the 
overstory from partial, selection, or two-age harvesting may produce few changes in the bird community (Medin and Booth, 1989; Annand and Thompson, 1997; Baker and Lacki, 1997; Duguay, 1997), whereas large disturbances such as clearcutting may result in the replacement of forest interior species with edge and interior-edge species (Annand and Thompson, 1997). Diameter-limit harvesting is a commonly used harvesting technique among landowners in West Virginia (Fajvan et al., 1998). However, little is known about the effects of this harvesting practice on breeding birds.

Diameter-limit harvesting is being used extensively in the eastern hardwood forest (Miller, 1993), including the central Appalachians, because this practice is preferred by landowners and producers for logistic and economic reasons. Over 35\% of West Virginia's nonindustrial private landowners, that own $>60 \%$ of forest land in this state, plan to cut their forests in the next 10 years, and many are expected to use the diameter-limit technique (Birch et al., 1992). Using this technique, trees bigger than a pre-determined diameter-limit are removed. The diameter-limit in eastern deciduous forests traditionally has been 30.5-cm (Miller, 1993). Many foresters consider diameter-limit harvesting to be exploitative, because trees are removed with little or no regard to silviculture and future conditions (Nyland, 1992). Trees are removed that have immediate market value, and often the stand is 'high graded' when only the highest quality trees are removed and inferior trees are left. The use of diamter-limit harvesting in West Virginia has led to reductions in average stand diameters and reductions in basal areas of commercially valuable tree species (Favjan et al., 1998). Short-term profits are greatest with this type of harvest as compared to selection harvests (Miller, 1993), but diameter-limit harvesting distorts the structural stability of the stand, and the landowner will have inconsistent yields at a level below those projected from selection harvesting (Nyland et al., 1993). Nevertheless, forest tree species regeneration and age structure after diameter-limit harvesting appears to resemble single-tree selection more closely than clearcutting (Trimble, 1973; Miller and Smith, 1991).

Because some canopy cover remains after a diameter-limit harvest, bird communities might not change as dramatically immediately after harvest as has been documented following clearcutting. However, diameter-limit harvesting has not been extensively examined for its effects on songbird population sizes or reproductive success. It has been shown that songbird abundance may not be a good indicator of habitat quality, and that nesting success also should be considered when managing for songbirds (Van Horne, 1983; Vickery et al, 1992). Accordingly, 
both abundance and nest survival should be considered when assessing the impact of forest management activities on songbirds. The primary focus of my research was to examine how songbird abundances and nesting success were affected in areas that were harvested using the diameter-limit technique where the minimum diameter at breast height ( $\mathrm{dbh}$ ) of harvested trees was $\geq 45.7-\mathrm{cm}$. My specific objectives were: 1) to compare songbird abundances among areas that had diameter-limit harvests, that were peripheral to diameter-limit harvests, and that were unharvested; 2) To compare nesting success among areas that had diameter-limit harvests, that were peripheral to diameter-limit harvests, and that were unharvested; 3 ) to examine how individual habitat characteristics important to songbirds changed after harvesting, and 4) to examine how habitat characteristics important to songbirds of unharvested and harvested areas differed.

\section{Methods:}

\subsection{Study area}

My study area was the Westvaco Wildlife and Ecosystem Research Forest (WWERF). The WWERF is a 3,360-ha area located in the Unglaciated Allegheny Mountain and Plateau physiographic province of West Virginia (Fenneman, 1938) in southwestern Randolph County Virginia $\left(38^{\circ} 42^{\prime} \mathrm{N}\right.$ lat., $80^{\circ} 3^{\prime} \mathrm{W}$ long.). Established by the Westvaco Corporation in 1994, the area is reserved for the study of industrial forestry impacts on ecosystems and ecological processes in an Appalachian setting. The oldest forests on the WWERF are second growth stands that were established by natural regeneration following wide-scale railroad logging that occurred in this portion of West Virginia in the 1900-1920's (Clarkson, 1993). Currently, the WWERF is undergoing a 10-year harvest schedule throughout the site comprised of a mix of diameter-limit harvests for sawtimber production, silvicultural clearcutting (seed tree harvests) for stand regeneration, and two-aged regeneration harvests. Elevations range from 740 to 1200m. Topography consists of steep side slopes with broad, plateau-like ridgetops and narrow valleys with small, high-gradient streams. The climate is cool and moist, with average annual precipitation exceeding 155-cm (Strausbaugh and Core, 1977). Soils on the area are acidic and well-drained inceptisols (Stephenson, 1993). Forest cover is primarily an Allegheny hardwoodnorthern hardwood type dominated by beech (Fagus grandifolia), yellow birch (Betula alleghaniensis), sugar maple (Acer saccharum) red maple (A. rubrum), black cherry (Prunus 
serotina), Fraser's magnolia (Magnolia fraseri), and red spruce (Picea rubens). Plant nomeclature follows Strausbaugh and Core (1977). At the lower elevations, species from the cove hardwood and mixed mesophytic associations, including tuliptree (Liriodendron tulipifera), basswood (Tilia americana), sweet birch (B. lenta), and northern red oak (Quercus rubra) occur. Riparian areas are characterized by hemlock (Tsuga canadensis)-red spruce-rhododendron (Rhododendron maximum) communities. Upland hardwoods, dominated by chestnut oak ( $Q$. prinus) and scarlet oak $(Q$. coccinea) occur on some ridges and xeric slopes. Red spruce and eastern hemlock also predominate on some exposed ridges. Throughout, rhododendron and striped maple (A. pensylvanicum) form dense understory thickets. As an artifact of past forest management activities and excessive deer herbivory, a dense coverage of hay-scented fern (Dennstaedtia punctilobula) occurs where the shrub layer is absent and the overstory canopy is not continuous.

\subsection{Songbird Abundance}

Point counts were conducted from 1996 to 1998 to determine songbird abundances at 79 stations within 44 forest stands. Stands were delineated by Westvaco personnel based on forest type, age, and percent stocking. Sizes ranged from 9.3-ha to 108.1-ha (average 40-ha), and the average age was 69 years (range 62-82). Point count stations were distributed throughout the forest types approximately equal to their proportion on the forest; $80 \%$ were in Appalachian hardwoods (beech-maple-birch), 12\% were in cove forest (tuliptree-basswood-red oak), and 9\% were in upland hardwoods (chestnut oak-scarlet oak-hemlock). Point count stations were spaced either $242 \mathrm{~m}$ (12 chains) or $484 \mathrm{~m}$ apart. All points were $>1000 \mathrm{~m}$ from any other harvest type, such as a seed tree cut, but minor disturbances such as forest roads with partial canopy closure were not avoided when establishing point counts. Previous analyses indicated that point count stations were spatially independent (Weakland and Wood, unpub. rept.).

Counts were conducted from 0545 to 1000 hours on $50 \mathrm{~m}$ radius plots from late May to the end of June using standardized methods (Ralph et al., 1993). Counts lasted $10 \mathrm{~min}$ and all birds detected by sight or sound within the $50 \mathrm{~m}$ radius were recorded to species. Gates (1995) determined that most birds in the central Appalachians are detected in the first 5 min of counting, therefore, two 10 min counts were adequate for sampling the majority of species present on the WWERF. Some rare species likely had low detection probabilities and were not adequately sampled, but detections of most of the common species likely were adequate for relative 
comparisons of abundance. Birds were not surveyed when it was rainy, foggy or when noise from wind interfered with counting. Two experienced observers surveyed each point count. To reduce observer bias, each observer visited all point count stations only once each year (Nur et al., 1999) All observers were trained in bird identification by sight and sound and distance estimation prior to beginning the surveys.

Point counts were placed into three treatments: harvested, peripheral to a harvest, and unharvested. Peripheral points were points $>50 \mathrm{~m}$ and $\leq 480 \mathrm{~m}$ from a harvest. These points were not included with unharvested points because songbirds returning to an area that has been harvested might move to the closest suitable habitat (Hagan et al., 1996). Nineteen points in 10 stands were harvested with the diameter-limit technique; 17 points in 14 stands were peripheral to a harvest, and 42 points in 37 stands were unharvested. In harvested stands, trees $\geq 45.7 \mathrm{~cm}$ were removed. This dbh is larger than the typical $30.5 \mathrm{~cm}$ used on private lands in the eastern United States (Miller, 1993). Before harvest, basal area averaged $22 \mathrm{~m}^{2} /$ ha, and the number of trees averaged 530 trees/ha. After harvested, basal area decreased to $20 \mathrm{~m}^{2} / \mathrm{ha}$, and the number of trees averaged 506 trees/ha

All bird species were classified into 1 of 3 habitat preference categories and 1 of 5 nest guild strategies using the criteria of Freemark and Collins (1992) and Ehrlich et al. (1988). Habitat use categories were edge, forest-interior, and interior-edge. Nesting guilds were ground, shrub, subcanopy, canopy, and cavity nesters. Total abundance, abundance of each habitat group and nesting guild, and of individual species observed at $\geq 20 \%$ of point counts were analyzed for differences among treatments. Species nomenclature follows the American Ornithologists' Union check-list of North American birds (American Ornithologists' Union 1999).

Least-squared means of post-harvest abundances were tested for differences among harvested, peripheral and unharvested points using analysis of covariance (ANCOVA) with pretreatment abundance as a covariate (Cody and Smith, 1997). Factor levels were treatment, stand, and year. Stand was nested within treatment to account for variability within stands. I tested for treatment effects and treatment by year interactions. If a treatment effect was detected, Scheffe's multiple comparison procedure was used to examine differences between least squared means (Neter et al., 1996). An important assumption in ANCOVA is that the slope of the regression line of the covariate by independent variable must be the same for all factor levels of the independent variable (Cody and Smith 1991). I tested for homogeneity of slopes before 
running the ANCOVA for each species and guild. If a species or guild showed a significant treatment by pre-harvest abundance interaction, then I did not use ANCOVA (Cody and Smith 1991). For these species, I used two-way nested ANOVA to compare treatments (Neter et al. 1996). Abundance estimates were transformed using the $\log _{10}($ variable +1$)$ transformation to help normalize residuals (Nur et al., 1999).

An index of vertical structure was included as a covariate in models for some species when there was a significant correlation between abundance and vertical structure, and when the relationship between abundance and vertical structure was homogenous among treatments (Cody and Smith, 1997). Vertical structure was included in the models because dense vegetation can attenuate bird songs and change detectability of some species; for example, songbird detection probabilities may be much different in clearcuts than mature forests (Pendleton, 1995). The vertical structure index was calculated using percent cover in 6 canopy layer classes: $\geq 0.5-3 \mathrm{~m}$, >3-6 m, >6-12 m, >12-18 m, >18-24 m, and >24 m (Nichols 1996). Percent canopy cover was determined using the sight-tube method of James and Shugart (1970). Four habitat subplots (Martin et al., 1997) were established within the $50 \mathrm{~m}$ radius circle of each point count plot (Fig. 2. 1). One subplot was centered on the point count station, and the other three were located $35 \mathrm{~m}$ away from plot center at $0^{\circ}, 120^{\circ}$, and $240^{\circ}$. Twenty presence/absence site-tube readings were recorded for each layer class along two $22.6 \mathrm{~m}$ transects that intersected at the center of each subplot (James and Shugart, 1970) (Fig. 2.1). The average canopy cover of these four subplots was used to calculate the vertical structure index. Measurements were taken at all point counts before harvesting and at all harvested points in 1998. Percentage data were subjected to arcsinesquare root transformations; tree and understory stem densities were converted using the transformation $\log _{10}($ variable+1) (Dowdy and Wearden, 1991).

\subsection{Nest Survival}

In the spring of 1996, four 40-ha (630-m x 630-m) nest monitoring plots (Fig. 2.1) were established on the WWERF using protocols established by the Breeding Biology Research and Monitoring Database Program (BBIRD; Martin et al. 1997). Two plots were located in stands such that approximately one-half of the plot had diameter-limit harvests between August 1996 and April 1997. The other two plots were not harvested and were left as controls. After harvesting, treated plots were divided into "harvested stands" and "peripheral stands". Each plot was marked with flagging every $60 \mathrm{~m}$ as an aid in relocating nests. Each plot was visited every 
2-3 days from 20 May -15 July by observers trained in nest searching and monitoring techniques (Martin and Guepel, 1993). Every nest found was checked every 3-4 days to determine clutch size, number of nestlings, and nest fate. High nests were checked with a mirror attached to a pole. Methods for locating nests and recording data followed BBIRD protocols. We examined nest survival on unharvested (control) plots and pre-harvest treatment plots in 1996. In 1997-98 nest survival was examined on unharvested plots, harvested areas of treatment plots, and peripheral areas of treatment plots.

Sample sizes for nests of individual species were low. We combined species to examine overall trends of nest survival. Daily nest survival and total nesting period survival for all species combined and for nesting guilds were calculated using the Mayfield method (Mayfield, 1961, 1975, Johnson 1979), where

$$
\begin{gathered}
\left.\mathrm{DS}_{\text {period }}=1 \text {-(number of failed nests/exposure days }\right) \text {, and } \\
\mathrm{S}_{\text {period }}=\text { daily survival }{ }^{\text {nest days }} \text {. }
\end{gathered}
$$

Exposure days were the number of days that a nest was under observation in each period (incubating or brooding) of the nest cycle. Nest days were the total number of days in a species' typical nesting cycle. Estimates were obtained for the incubation and brood periods separately because there may be differential nesting mortality in each of the two periods. Total survival estimates for the entire nesting period were then determined by multiplying $S_{\text {incubation }}$ by $S_{\text {brood }}$ (Mayfield, 1975). The egg-laying period was not included due to small sample sizes. Nest day values for each period for each species were acquired from the BBIRD database or from my own data from the WWERF. To determine the survival estimates for all species combined, I used the average of the typical nest days for each species present in a treatment, and to determine the survival for guilds, I used the average of the typical nest days of all the species in the guild for each treatment. Exposure days, daily survival rates, and total survival for all nests combined, and for individual guilds are presented for each treatment, but these values were not compared statistically because of small sample sizes.

\subsection{Habitat Characteristics}

Habitat characteristics were examined before and after harvesting. On each habitat subplot on nest searching plots, I counted the number of shrubs, saplings and poles within a 5.0 $\mathrm{m}$ radius circle of the subplot center (Fig. 2.1). Shrubs were defined as any woody species $\geq 0.5$ $\mathrm{m}-1.4 \mathrm{~m}$ high, and saplings were defined as any woody species $>1.4-\mathrm{m}$ high but $<2.5-\mathrm{cm}$ at 10 
$\mathrm{cm}$ above the ground (Martin et al., 1997). Poles were trees $\geq 2.5-7.9 \mathrm{~cm} \mathrm{dbh}$. All larger trees were counted within the 11.3-m radius subplot and placed into one of five size classes: >8-14.9 $\mathrm{cm},>15-22.9 \mathrm{~cm},>23-29.9 \mathrm{~cm},>30-37.9 \mathrm{~cm}$, and $>38 \mathrm{~cm}$ dbh (Martin et al., 1997). In addition, all snags $>1.4 \mathrm{~m}$ high and $\geq 12.0 \mathrm{~cm}$ dbh were counted within the $11.3 \mathrm{~m}$ radius circle (Martin et al., 1997). Percent ground cover estimates were taken in the same manner as canopy cover estimates (James and Shugart, 1970). Ground cover categories were green vegetation, down wood, bareground/rock, and litter. Green vegetation included grasses, ferns, herbaceous plants, moss, and trees $<0.5 \mathrm{~m}$ high. Down wood was any $\log$ that was $\geq 4.0 \mathrm{~cm}$ in diameter. Smaller woody debris was counted as litter. All measurements were taken the growing season before harvesting (1996) and two growing seasons after harvesting (1998). The averages of all measurements on the four subplots were used in statistical testing.

I examined differences in habitat structure between years at harvested points using twosample Wilcoxon rank sum tests (Hollander and Wolfe, 1973). Nested ANOVA was used to examine differences between unharvested and harvested points in 1998. Treatment was the main effect with stand nested within treatment.

The $\mathrm{SAS}^{\circledR}$ GLM procedure $\left(\mathrm{SAS}^{\circledR}\right.$ Institute, 1991) was used to conduct all ANCOVA and ANOVA tests; and $\mathrm{JMP}^{\circledR}\left(\mathrm{SAS}^{\circledR}\right.$ Institute, 1995) was used to conduct Wilcoxon rank sum tests. Differences were considered significant at $\mathrm{P} \leq 0.10$ for all main-effects models. The conservative P-value of 0.10 was chosen in order to minimize the probability of a Type II error, which may be more important in ecological situations than minimizing a Type I error (Askins et al., 1990; Johnson, 1999). Error rates were controlled for all treatment comparisons for a bird species, but not experiment-wide for all species. Scheffe' multiple comparison tests were conducted with a family confidence level of 0.90 (Neter et al., 1996).

\section{Results:}

\subsection{Songbird abundance}

Abundance of ground nesters $(\mathrm{R}=-0.36, \mathrm{P}=0.01)$, shrub nesters $(\mathrm{R}=0.25, \mathrm{P}=0.03)$, interior-edge species $(\mathrm{R}=-0.28, \mathrm{P}=0.01)$, and total abundance $(\mathrm{R}=-0.25, \mathrm{P}=0.02)$ were weakly correlated to vertical structure. Abundances of three species, Black-throated blue warblers (Dendroica caerulescens; $\mathrm{R}=0.31, \mathrm{P}=0.01$ ), Dark-eyed juncos (Junco hyemalis; $\mathrm{R}=-0.29$, $\mathrm{P}=0.01$ ), and Canada warblers (Wilsonia canadensis; $\mathrm{R}=-0.28, \mathrm{P}=0.01$ ) also were weakly 
correlated to vertical structure. The relationship between abundance and vertical structure was found to be homogenous among treatments for all of these guilds and species except for interioredge species and Canada warblers. Thus, vertical structure was included in the ANCOVA models for these guilds/species. There was a significant interaction between pre-harvest abundance and treatment for Black-throated blue warblers, Canada warblers, and Dark-eyed juncos, thus pre-harvest abundance was not included as a covariate in models for these species.

Results indicated that total songbird abundance was higher in harvested areas than in peripheral and unharvested areas $(\mathrm{F}=3.34, \mathrm{P}=0.04)$ (Fig. 2.2). One habitat group, interior-edge species showed treatment effects (Table 2.1). Abundance of interior-edge species was higher in harvested areas than in peripheral and unharvested areas. Cavity nesters also were higher in diameter-limit harvests than in peripheral and unharvested areas (Table 2.1). Only one guild, subcanopy nesters, showed a treatment by year interaction. Abundance of this group was higher in harvested areas than peripheral or unharvested areas in 1997, but not in 1998.

Nine species were detected on $>20 \%$ of point count plots. Abundances of the Canada warbler, Dark-eyed junco, and Magnolia warbler (Dendroica magnolia) showed significant treatment effects (Table 2.2). The Canada warbler was more abundant in harvested areas than in unharvested areas; the Dark-eyed junco was more abundant in harvested areas than in unharvested and peripheral areas; and the Magnolia warbler was more abundant in peripheral areas than unharvested areas. Treatment by year interactions were not apparent for any species.

\subsection{Nest Survival}

Nests of 23 species (Table 2.3) were found and monitored during this study. Thirty-three nests were located in 1996, before harvesting (Table 2.3). Of these, 13 were on control (unharvested) plots and 20 were on pre-harvest plots. In the two years after harvesting, 25 nests were found on control plots, 17 nests were located in the harvested portion of treatment plots, and 18 nests were located in the peripheral portion of treatment plots (Table 2.3). The majority were ground nests $(60 \%)$. Few nests of shrub (8\%), subcanopy (26\%), canopy (4\%), or cavity nesters $(2 \%)$ were found in all three years (Table 2.3).

Overall nesting success on control plots was $37.2 \%$ in 1996, 14.2\% in 1997 and $28.4 \%$ in 1998 (Table 2.4). In contrast, nesting success on pre-harvest plots was $14.9 \%$ in 1996. Nesting success in harvested areas was 65.0\% in 1997 and 50.3\% in 1998. Survival in peripheral areas was $35.7 \%$ in 1997 and $46.4 \%$ in 1998 (Table 2.4). 
No nests monitored were parasitized by Brown-headed cowbirds (Molothrus ater). On unharvested plots, $72.7 \%$ of nest failures were attributable to predation, $9 \%$ were abandoned, $4.5 \%$ were lost because of weather, and $13.7 \%$ failed because of undetermined reasons. On preharvest treatment plots, $64.3 \%$ of nest failures were caused by predation, $7.1 \%$ were abandoned, $7.1 \%$ failed because of human error, $7.1 \%$ were trampled by deer, and $14.3 \%$ failed because of undetermined reasons. On harvested treatment plots, $87.5 \%$ failed because of predation and $12.5 \%$ failed because of unknown reasons.

\subsection{Habitat structure}

Five habitat variables differed before and after harvesting at harvested plots (Fig. 2.3). Canopy cover from $\geq 0.5-3 \mathrm{~m}(\mathrm{~W}=-19.5, \mathrm{P}=0.047)$ nearly doubled, and the percent cover of bareground/rock $(\mathrm{W}=-17.5, \mathrm{P}=0.035)$ nearly tripled after the harvest, whereas canopy cover $>24$ $\mathrm{m}(\mathrm{W}=19.5, \mathrm{P}=0.020)$, the percent cover of litter $(\mathrm{W}=24.5, \mathrm{P}=0.010)$, and the density of saplings $(\mathrm{W}=19.5, \mathrm{P}=0.020)$ decreased after the harvest (Fig. 2.3). The vertical structure index changed slightly after harvesting, from an average of 10.70 to 9.77 , but this change was not significant $(\mathrm{W}=11.0, \mathrm{P}=0.283)$.

Four habitat variables differed between unharvested and harvested plots in 1998 (Table 2.5). Percent cover of down wood, density of trees $>8-14.9 \mathrm{~cm} \mathrm{dbh}$, and density of snags were greater in the harvested treatment, whereas sapling density was greater in unharvested stands (Table 2.5). All other variables did not differ between harvested and unharvested plots. The vertical structure index averaged 9.77 at harvested points and 10.48 at unharvested plots; this difference was not significant $(\mathrm{F}=0.20, \mathrm{P}=0.663)$.

\section{Discussion:}

Although diameter-limit harvesting is not an accepted silvicultural system, it is being used extensively to harvest Appalachian hardwood forests (Miller, 1993). Consequently, it is important to identify the effects of this harvesting practice on avian communities and vegetation structure. In my study, most (but not all) trees $\geq 45.7 \mathrm{~cm}$ dbh were cut resulting in stands that differed from unharvested forests in only three characteristics: number of snags, number of trees $\geq 8-15 \mathrm{~cm}$., and percent cover of down wood. Thus, the vegetation structure that I measured changed little after harvesting with a $45.7 \mathrm{~cm}$ diameter-limit. Canopy cover, stem density of 
canopy trees, and vegetation vertical structure did not differ between harvested and unharvested forest.

Abundance of most songbird species did not differ among unharvested, peripheral, and harvested areas or among years at harvested points. My results suggest that a diameter-limit harvest of $45.7-\mathrm{cm}$ does not have significant negative impacts on most songbird species I encountered immediately after harvesting. My results were similar to other studies that have examined songbird abundance in relation to harvesting practices that leave residual trees in the overstory (Medin and Booth, 1989; Lent and Capen, 1995; Annand and Thompson, 1997; Baker and Lacki, 1997; Hagan et al., 1997; Chambers et al., 1999; Duguay, 1997). However, my data were limited to two breeding seasons post-harvest and do not provide information on long-term impacts on forest interior species or canopy- and subcanopy-nesting species. Additionally, I only present results for the most common species on the WWERF. Less common species that nest in the canopy, such as the Blackburnian warbler (Dendroica fusca) and Golden-crowned kinglet (Regulus satrapa) might respond differently to harvesting than the species I examined.

The bird community changed little after single-tree selection in Idaho (Medin and Booth, 1989). Two species examined in that study also were observed in my study: the Blue-headed vireo and the Dark-eyed junco. Similar to my study, they found that abundance of Blue-headed vireos was not significantly different between logged and uncut sites (Table 2.6). However, I observed higher abundance of Dark-eyed juncos in harvested areas than in peripheral and unharvested areas. Lent and Capen (1995) mapped songbird territories in Vermont and found that some species, including the Magnolia warbler, preferred small clearcuts and selection cuts. I found that this species was more abundant in peripheral areas than unharvested areas, but abundance in harvested areas did not differ from peripheral or unharvested areas. Other species showed no response to harvests (Lent and Capen, 1995). Species-specific responses to timber harvesting are similar among regions and harvest types (Annand and Thompson, 1997; Chambers et al., 1999).

I detected a positive response to the diameter-limit harvest by Canada warblers and Dark-eyed juncos. The increase in low cover $(\geq 0.5-3 \mathrm{~m})$ possibly led to increases in Canada warblers. They were often found foraging, singing, and nesting in low-growing vegetation. Dark-eyed juncos frequently were found nesting in the earthen banks of logging roads, which is a possible reason for their positive response to harvesting. In addition, no guild or species 
appeared to be negatively affected by diameter-limit harvesting on the WWERF. Interior-edge species were more abundant on harvested sites than peripheral and unharvested sites, whereas forest-interior species and the other nesting guilds did not differ among treatments. Interior-edge species possibly were responding to increased edge habitat created by logging roads and canopy openings.

Similar results were found by Bell (1998) in a forest defoliated by gypsy moth catepillars (Lymantria dyspar). Gypsy moth defoliation decreases the number of overstory oaks and releases understory vegetation (Fajvan and Wood, 1996). Early successional songbird species respond positively to this disturbance (Bell and Whitmore, 1997; Bell 1998). It is possible that diameter-limit harvesting mimics the disturbance pattern observed in oak forests defoliated by gypsy moths. However, few oaks occurred on my diameter-limit stands before or after harvesting, and my results may not be directly comparable to Bell (1998). Additionally, gypsy moths are an introduced species to North America and the pattern of defoliation by this species may not necessarily be considered a "natural" disturbance event.

Few published studies have examined nesting success of songbirds in relation to timber harvesting practices in the central Appalachians. Nest survival in two-age, clearcut, and unharvested stands on the Monongahela National Forest (MNF) in West Virginia were examined by Duguay et al. (in press). Nests in unharvested areas had higher daily survival than those in two-age harvests, but daily survival in clearcuts did not differ from survival in unharvested areas or in two-age harvests. It was found that nest predation was higher in forested areas than in open and regenerating areas in Minnesota (Hanski et al. 1996). These studies suggest that nest survival rates can vary by harvest type. In contrast, my results suggest that harvested areas had higher nest survival rates than unharvested areas. However, unharvested stands on the WWERF may differ from the unharvested stands studied by Duguay et al. (in press) and Hanski et al. (1996). Stands on the WWERF have been disturbed since 1920 on a periodic basis, and the overall amount of roads, trails, and edges on the WWERF might be higher than in their studies. The higher amount of linear edge on the WWERF might have contributed to the lower nest survival rates in unharvested areas. Thus, results from my study might not be directly comparable to theirs. In addition, I was unable to determine species-specific survival rates, which may vary among studies and habitats, and my sample size was low. Thus, my results might be caused by chance. 


\section{Conclusions}

Forest harvesting cannot be generalized as either detrimental or beneficial to songbirds (Crawford et al., 1981). Some species will respond positively to harvesting whereas others will be negatively affected. Fine-scale disturbances such as single-tree harvests allow mature forest bird species to persist and also provide habitat for early successional bird species. Gaps created by these harvests will increase heterogeneity across the landscape and increase vertical structure, which is expected to benefit not only songbirds but also other wildlife species.

In the short-term, the forest bird community on the WWERF, a managed, industrial forest, appeared to be retained after diameter-limit harvesting of trees $\geq 45.7-\mathrm{cm} \mathrm{dbh}$. With a large diameter-limit such as this, canopy cover and vegetation structure did not differ between unharvested and harvested stands. Consequently, I found no negative responses by species or guilds. However, many landowners often harvest trees to a smaller diameter-limit, traditionally $30.5 \mathrm{~cm}$ (Miller, 1993). In areas where smaller trees are harvested, species that favor closed canopies may still be present (Crawford et al., 1981). Species that prefer intermediate canopy closure and intermediate-sized trees will likely benefit from this type of harvest (Crawford et al., 1981). Data on avian response to these more intensive harvests are still needed. Because my study was short-term in nature and sample sizes for nests were low, my data cannot predict how diameter-limit harvesting will affect long-term trends in population sizes. Future research should focus on harvests with smaller diameter limits and should be long-term in nature in order to examine effects of yearly variation on population abundance.

\section{Ackowledgements}

This research was funded by Westvaco Corporation, Forest Resources Division, and the West Virginia Division of Natural Resources Nongame Wildlife Program. The West Virginia Cooperative Fish and Wildlife Research Unit, USGS, BRD, provided equipment and logistical support. I also thank the many field technicians who assisted with nest searching, point count surveys, and habitat sampling. R. C. Whitmore, L. S. Gribko, A. F. Egan, and W. M. Ford provided helpful suggestions on early drafts of this manuscript. 


\section{References}

American Ornithologists' Union, 1999. The American Ornithologists' Union check-list of North American birds. http://pica.wru.umt.edu/AOU/birdlist.HTML.

Annand, E.M., Thompson, F.R., III, 1997. Forest bird response to regeneration practices in central hardwood forests. J. Wildl. Manage. 61, 159-171.

Askins, R.A., Lynch, J.F., Greenburg, R., 1990. Population declines in migratory birds in eastern North America. Curr. Ornith. 7, 1-57.

Baker, M.D., Lacki, M.J., 1997. Short-term changes in bird communities in response to silvicultural prescriptions. For. Ecol. Manage. 96, 27-36.

Bell, J. L. 1998. Effects of gypsy moth defoliation on forest vegetation and a forest bird community. Ph. D. Dissertation, West Virginia University. 113pp.

Bell, J.L., Whitmore, R. C., 1997. Eastern towhee numbers increase following defoliation by gypsy moths. Auk 114, 708-716.

Birch, T.W., Gasner, D.A., Arner, S.L., Widmann, R.H., 1992. Cutting activity on West Virginia timberlands. North. J. Appl. For. 9, 146-148.

Chambers, C.L., McComb, W.C., Tappeiner, J.C., II, 1999. Breeding bird response to three silvicultural treatments in the Oregon coast range. Ecol. Appl. 91, 171-185.

Clarkson, R.B., 1993. Destruction of the upland forest by lumbering and fire. In: Stephenson, S.L. (Ed.), Upland Forests of West Virginia. McCain Printing Co., Parsons, West Virginia, pp. 35-46.

Cody, R.P., Smith, J.K., 1997. Applied statistics and the SAS programming language. Prentice Hall, Upper Saddle River, New Jersey, 445pp.

Crawford, H.S., Hooper, R.G., Titterington, R.W., 1981. Songbird population rsponse to silvicultural practices in central Appalachian hardwoods. J. Wildl. Manage. 45, 680692.

Dowdy, S., Wearden, S., 1991. Statistics for research. John Wiley and Sons. New York, New York, 629pp.

Duguay, J. P., 1997. Influence of two-age and clearcut timber management practices on songbird abundance, nest success, and invertebrate biomass in West Virginia. Ph.D. Dissertation, West Virginia University. 162pp. 
Duguay, J.P., Wood, P.B., Miller, G.W., in press. Effects of timber harvests on invertebrate biomass and avian nest success. Wildl. Soc. Bull.

Ehrlich, P.R., Dobkin, D.S., Wheye, D., 1988. The Birder's Handbook. Simon and Schuster Inc. New York, New York, 785pp.

Fajvan, M. A., Grushecky, S. T., Hassler, C. C., 1998. The effects of harvesting practices on West Virginia's wood supply. J. For. 96 (5), 33-39.

Fajvan, M. A., Wood, J. M., 1996. Stand structure and development after gypsy moth defoliation in the Appalachian plateau. For. Ecol. Man. 89, 79-88.

Fenneman, N.M., 1938. Physiography of the Eastern United States. McGraw-Hill, New York, New York, 714pp.

Freemark, K., Collins, B., 1992. Landscape ecology of breeding birds in temperate forest fragments. In: Hagan, J.M. and Johnston, D.W. (Eds.), Ecology and Conservation of Neotropical Migrant Landbirds. Smithsonian Institution Press, Washington and London, pp. 443-454.

Gates, J. E., 1995. Point count modifications and breeding bird abundances in central Appalachian forests. In: Ralph, C., Sauer, J. R., and Droege, S. (Eds.), Monitoring Bird Populations By Point Counts. U.S. For. Serv. Gen. Tech. Rep. PSW-GTR-149, pp.135144.

Hagan, J.M., Vander Haegen, W.M., McKinley, P.S., 1996. The early development of forest fragmentation effects on birds. Conserv. Biol. 10, 188-202.

Hagan, J.M., McKinley, P.S., Meehan, A.L., Grove, A.L., 1997. Diversity and abundance of landbirds in a northeastern industrial forest. J. Wildl. Manage. 61, 718-735.

Hanski, I.K., Fenske, T.J., Niemi, G.J., 1996. Lack of edge effect in nesting success of breeding birds in managed forest landscapes. Auk 113, 578-585.

Hollander, M., Wolfe, D.A., 1973. Nonparametric statistical methods. John Wiley and Sons, New York, New York. 503pp.

James, F.C., Shugart, H.H., 1970. A quantitative method of habitat description. Aud. Field Notes 24, 727-736.

Johnson, D. H., 1979. Estimating nest success the Mayfield method and an alternative. Auk 96, 651-661. 
Johnson, D.H., 1999. The insignificance of statistical significance testing. J. Wildl. Manage. $63,763-772$.

Lent, R.A., Capen, D.E., 1995. Effects of small-scale habitat disturbance on the ecology of breeding birds in a Vermont (USA) hardwood forest. Ecography 18, 97-108.

Martin, T.E., Guepel, G.R., 1993. Nest-monitoring plots: methods for locating and monitoring success. J. Field. Ornith. 64, 507-519.

Martin, T.E., Paine, C., Conway, C.J., Hochachka, W.M., Allen, P., Jenkins, W., 1997. BBIRD Field Protocol. USGS, Biological Resources Division, Montana Cooperative Fish and Wildlife Research Unit, Missoula, Montana.

Mayfield, H.F., 1961. Nesting success calculated from exposure. Wilson Bull. 73, 255-261.

Mayfield, H.F., 1975. Suggestions for calculating nest success. Wilson Bull. 87, 456-466.

Medin, D.E., Booth, G.D., 1989. Responses of birds and small mammals to single-tree selection logging in Idaho. U.S. For. Serv. Res. Pap. INT-408.

Miller, G.W., 1993. Financial aspects of partial cutting practices in central Appalachian hardwoods. U.S. For. Serv. Res. Pap. NE-673.

Miller, G.W., Smith, H.C., 1991. Comparing partial cutting practices in central Appalachian hardwoods. In: McCormick, L.H., and Gottscalk, K.W. (Eds.), Proc. $8^{\text {th }}$ Central Hardwood Forest Conference. Gen. Tech. Rep. NE-148, pp. 105-119.

Neter, J., Kutner, M. H., Nachtsheim, C. J., Wasserman, W., 1996. Applied Linear Statistical Models. Irwin. Homewood, Illinois. 1181pp.

Nichols, J.V., 1996. Effects of two-age timber management and clearcutting on songbird density and reproductive success. M.S. Thesis, West Virginia University. 131pp.

Nur, N., Jones, S.L., Geupel, G.R., 1999. A statistical guide to data analysis of avian monitoring programs. U.S. Fish and Wildl. Serv. Biol. Tech. Publ. BTP-R6001-1999.

Nyland, R. D. 1992. Exploitation and greed in eastern hardwood forests. J. For. 90 (1), 33-37.

Nyland, R.D., Alban, L.M., Nissen, R.L., Jr., 1993. Greed or sustention: silviculture or not. In: Briggs, R.D., and Krohn, W.B. (Eds.), Proc. Joint Meeting New England Society of American Foresters and Maine Chapter of the Wildlife Society. Maine Agr. For. Exp. Station, Misc. Rept. 382, SAF Pub. No. 93-05.

Pendleton, G. W., 1995. Effects of sampling strategy, detection probability, and independence of counts on the use of point counts. In: Ralph, C., Sauer, J. R., and Droege, S. (Eds.), 
Monitoring Bird Populations By Point Counts. U.S. For. Serv. Gen. Tech. Rep. PSWGTR-149, pp. 131-134.

Ralph, C.J., Guepel, G.R., Pyle, P., Martin, T.E., DeSante, D.F., 1993. Handbook of field methods for monitoring landbirds. U.S. For. Serv. Gen Tech. Rept. PSW-GTR-144.

Sas Institute Inc., 1991. SAS/STAT Guide for personal computers, version 6 edition. SAS Institute, Inc. Cary, North Carolina, 1028pp.

Sas Institute Inc., 1995. JMP Statistics and graphics guide, version 3.1. SAS Institute, Inc. Cary, North Carolina, 593pp.

Stephenson, S.L., 1993. An introduction to the upland forest region. In: Stephenson, S.L. (Ed.), Upland Forests of West Virginia. McCain Printing Co., Parsons, West Virginia, pp. 1-9.

Strausbaugh, P.D., and Core, E.L., 1977. Flora of West Virginia. Seneca Books, Grantsville, West Virginia. 1079 pp.

Trimble, G.R., Jr., 1973. The regeneration of central Appalachian hardwoods with emphasis on the effects of site quality and harvesting practice. U.S. For. Serv. Res. Pap. NE-282.

Van Horne, B., 1983. Density as a misleading indicator of habitat quality. J. Wildl. Manage. 47, 893-901.

Vickery, P.D., Hunter, M.L., Jr., Wells, J.V., 1992. Is density an indicator of breeding success? Auk 109, 706-710. 
Table 2.1. Means and standard errors (SE) for the abundance of nesting guilds and habitat groups at harvested, peripheral, and unharvested stands the breeding season before harvest (1996) and two breeding seasons after (1997-98) on the Westvaco Wildlife and Ecosystem Research Forest, Randolph County, West Virginia. Means are for untransformed data and are not adjusted for other variables in ANCOVA models.

\begin{tabular}{|c|c|c|c|c|c|c|c|c|c|c|c|}
\hline \multirow[b]{4}{*}{ Guild } & \multicolumn{11}{|c|}{ Treatment } \\
\hline & \multicolumn{3}{|c|}{ Harvested } & \multicolumn{3}{|c|}{ Peripheral } & \multicolumn{3}{|c|}{ Unharvested } & \multirow{2}{*}{\multicolumn{2}{|c|}{$\begin{array}{c}\text { ANCOVA }^{\mathrm{a}} \\
\text { Results }^{\mathrm{a}}\end{array}$}} \\
\hline & 1996 & 1997 & 1998 & 1996 & 1997 & 1998 & 1996 & 1997 & 1998 & & \\
\hline & $\begin{array}{c}\text { Mean } \\
(\mathrm{SE})\end{array}$ & $\begin{array}{c}\text { Mean } \\
(\mathrm{SE})\end{array}$ & $\begin{array}{c}\text { Mean } \\
(\mathrm{SE})\end{array}$ & $\begin{array}{c}\text { Mean } \\
(\mathrm{SE})\end{array}$ & $\begin{array}{c}\text { Mean } \\
(\mathrm{SE})\end{array}$ & $\begin{array}{c}\text { Mean } \\
(\mathrm{SE})\end{array}$ & $\begin{array}{c}\text { Mean } \\
(\mathrm{SE})\end{array}$ & $\begin{array}{c}\text { Mean } \\
(\mathrm{SE})\end{array}$ & $\begin{array}{c}\text { Mean } \\
(\mathrm{SE})\end{array}$ & $\mathrm{F}$ & $\mathrm{P}$-value \\
\hline \multicolumn{12}{|l|}{ Nesting: } \\
\hline \multirow[t]{2}{*}{ Ground } & 1.39 & 3.05 & 2.00 & 1.50 & 1.94 & 1.53 & 1.59 & 1.89 & 1.66 & 0.86 & 0.43 \\
\hline & $(0.28)$ & $(0.58)$ & $(0.28)$ & $(0.40)$ & $(0.41)$ & $(0.29)$ & $(0.13)$ & $(0.22)$ & $(0.21)$ & & \\
\hline \multirow[t]{4}{*}{ Shrub } & 0.39 & 0.22 & 0.33 & 0.56 & 0.88 & 0.59 & 0.66 & 0.57 & 0.48 & 0.37 & 0.69 \\
\hline & $(0.14)$ & $(0.13)$ & $(0.11)$ & $(0.18)$ & $(0.23)$ & $(0.21)$ & $(0.13)$ & $(0.15)$ & $(0.11)$ & & \\
\hline & & & & & & & & & & 1997: & \\
\hline & & & & & & & & & & 5.45 & $<0.01$ \\
\hline \multirow[t]{2}{*}{ Subcanopy $^{\mathrm{b}}$} & 1.72 & 2.28 & 1.89 & 0.69 & 1.18 & 1.82 & 1.31 & 1.34 & 1.98 & 1998: & \\
\hline & $(0.27)$ & $(0.30)$ & $(0.20)$ & $(0.15)$ & $(0.21)$ & $(0.26)$ & $(0.14)$ & $(0.11)$ & $(0.15)$ & 0.11 & 0.89 \\
\hline \multirow[t]{2}{*}{ Canopy } & 1.94 & 2.94 & 1.94 & 1.75 & 2.36 & 1.59 & 1.54 & 2.48 & 1.54 & 1.30 & 0.28 \\
\hline & $(0.34)$ & $(0.31)$ & $(0.26)$ & $(0.28)$ & $(0.30)$ & $(0.24)$ & $(0.12)$ & $(0.24)$ & $(0.18)$ & & \\
\hline \multirow[t]{2}{*}{ Cavity $^{\mathrm{c}}$} & 0.39 & 0.83 & 0.89 & 0.25 & 0.18 & 0.47 & 0.38 & 0.52 & 0.45 & 3.23 & 0.05 \\
\hline & $(0.16)$ & $(0.17)$ & $(0.25)$ & $(0.11)$ & $(0.09)$ & $(0.17)$ & $(0.10)$ & $(0.09)$ & $(0.11)$ & & \\
\hline
\end{tabular}


Table 2.1. Continued.

\begin{tabular}{|c|c|c|c|c|c|c|c|c|c|c|c|}
\hline \multirow[b]{4}{*}{ Guild } & \multicolumn{11}{|c|}{ Treatment } \\
\hline & \multicolumn{3}{|c|}{ Harvested } & \multicolumn{3}{|c|}{ Peripheral } & \multicolumn{3}{|c|}{ Unharvested } & \multirow{2}{*}{\multicolumn{2}{|c|}{$\begin{array}{l}\text { ANCOVA }^{\mathrm{a}} \\
\text { Results }^{\mathrm{a}}\end{array}$}} \\
\hline & 1996 & 1997 & 1998 & 1996 & 1997 & 1998 & 1996 & 1997 & 1998 & & \\
\hline & $\begin{array}{c}\text { Mean } \\
(\mathrm{SE})\end{array}$ & $\begin{array}{c}\text { Mean } \\
(\mathrm{SE})\end{array}$ & $\begin{array}{c}\text { Mean } \\
(\mathrm{SE})\end{array}$ & $\begin{array}{c}\text { Mean } \\
(\mathrm{SE})\end{array}$ & $\begin{array}{c}\text { Mean } \\
(\mathrm{SE})\end{array}$ & $\begin{array}{c}\text { Mean } \\
(\mathrm{SE})\end{array}$ & $\begin{array}{c}\text { Mean } \\
(\mathrm{SE})\end{array}$ & $\begin{array}{l}\text { Mean } \\
(\mathrm{SE})\end{array}$ & $\begin{array}{c}\text { Mean } \\
(\mathrm{SE})\end{array}$ & $\mathrm{F}$ & $\mathrm{P}$-value \\
\hline \multicolumn{12}{|l|}{ Habitat: } \\
\hline \multirow[t]{2}{*}{ Edge $^{d}$} & 0.67 & 0.55 & 0.22 & 0.44 & 0.47 & 0.41 & 0.33 & 0.50 & 0.23 & 2.29 & 0.11 \\
\hline & $(0.23)$ & $(0.22)$ & $(0.10)$ & $(0.16)$ & $(0.21)$ & $(0.26)$ & $(0.08)$ & $(0.11)$ & $(0.08)$ & & \\
\hline Interior/ & 1.61 & 3.22 & 3.00 & 1.25 & 1.35 & 2.00 & 1.55 & 1.82 & 2.04 & 5.91 & $<0.01$ \\
\hline $\operatorname{Edge}^{\mathrm{c}}$ & $(0.23)$ & $(0.61)$ & $(0.34)$ & $(0.27)$ & $(0.36)$ & $(0.30)$ & $(0.15)$ & $(0.16)$ & $(0.17)$ & & \\
\hline Forest & 3.28 & 5.05 & 3.39 & 3.12 & 5.00 & 4.29 & 3.17 & 4.07 & 3.41 & 2.30 & 0.11 \\
\hline Interior & $(0.39)$ & $(0.30)$ & $(0.33)$ & $(0.43)$ & $(0.54)$ & $(0.48)$ & $(0.16)$ & $(0.29)$ & $(0.29)$ & & \\
\hline
\end{tabular}

${ }^{a}$ ANCOVA, test for treatment effects in 1997-98 with pre-harvest abundance as a covariate.

${ }^{\mathrm{b}}$ Significant treatment by year interaction.

c Abundance significantly higher in harvested areas than peripheral and unharvested areas (Scheffe's multiple comparison test, family alpha level=0.10).

c Abundance significantly higher in harvested areas than peripheral areas (Scheffe's multiple comparison test, family alpha level= $0.10)$. 
Table 2.2. Means and standard errors (SE) for songbird abundance (species at $\geq 20 \%$ of point counts) at harvested, peripheral, and unharvested stands the breeding season before (1996) harvest and two breeding seasons after (1997-98) on the Westvaco Wildlife and Ecosystem Research Forest, Randolph County, West Virginia. Means are for untransformed data and are not adjusted for other variables in ANCOVA models.

\begin{tabular}{|c|c|c|c|c|c|c|c|c|c|c|c|}
\hline \multirow[b]{4}{*}{ Species } & \multicolumn{11}{|c|}{ Treatment } \\
\hline & \multicolumn{3}{|c|}{ Harvested } & \multicolumn{3}{|c|}{ Peripheral } & \multicolumn{3}{|c|}{ Unharvested } & \multirow{2}{*}{\multicolumn{2}{|c|}{$\begin{array}{c}\text { ANOVA } \\
\text { Results }\end{array}$}} \\
\hline & 1996 & 1997 & 1998 & 1996 & 1997 & 1998 & 1996 & 1997 & 1998 & & \\
\hline & $\begin{array}{l}\text { Mean } \\
(\mathrm{SE})\end{array}$ & $\begin{array}{l}\text { Mean } \\
(\mathrm{SE})\end{array}$ & $\begin{array}{l}\text { Mean } \\
(\mathrm{SE}) \\
\end{array}$ & $\begin{array}{l}\text { Mean } \\
(\mathrm{SE}) \\
\end{array}$ & $\begin{array}{c}\text { Mean } \\
(\mathrm{SE}) \\
\end{array}$ & $\begin{array}{l}\text { Mean } \\
(\mathrm{SE})\end{array}$ & $\begin{array}{l}\text { Mean } \\
(\mathrm{SE})\end{array}$ & $\begin{array}{c}\text { Mean } \\
(\mathrm{SE})\end{array}$ & $\begin{array}{c}\text { Mean } \\
(\mathrm{SE}) \\
\end{array}$ & $\mathrm{F}$ & P-value \\
\hline \multicolumn{12}{|l|}{ Black-throated } \\
\hline \multicolumn{12}{|l|}{ Blue warbler } \\
\hline (Dendroica & 0.28 & 0.17 & 0.28 & 0.31 & 0.53 & 0.35 & 0.32 & 0.20 & 0.25 & 0.85 & 0.43 \\
\hline caerulescens) & $(0.13)$ & $(0.12)$ & $(0.11)$ & $(0.12)$ & $(0.17)$ & $(0.15)$ & $(0.07)$ & $(0.07)$ & $(0.07)$ & & \\
\hline \multicolumn{12}{|l|}{ Black-throated } \\
\hline green warbler & 0.83 & 1.05 & 0.44 & 0.50 & 0.65 & 0.47 & 0.41 & 0.95 & 0.70 & 1.04 & 0.36 \\
\hline (D. virens) & $(0.20)$ & $(0.22)$ & $(0.14)$ & $(0.18)$ & $(0.19)$ & $(0.15)$ & $(0.07)$ & $(0.13)$ & $(0.10)$ & & \\
\hline \multicolumn{12}{|l|}{ Blue-headed } \\
\hline vireo (Vireo & 0.83 & 1.39 & 0.72 & 0.56 & 1.35 & 0.29 & 0.89 & 0.91 & 0.36 & 1.41 & 0.25 \\
\hline solitarius) & $(0.12)$ & $(0.16)$ & $(0.16)$ & $(0.13)$ & $(0.23)$ & $(0.11)$ & $(0.11)$ & $(0.14)$ & $(0.09)$ & & \\
\hline \multicolumn{12}{|c|}{ Canada warbler ${ }^{\mathrm{b}}$} \\
\hline (Wilsonia & 0.17 & 1.17 & 0.78 & 0.56 & 0.82 & 0.59 & 0.64 & 0.50 & 0.41 & 5.63 & 0.01 \\
\hline canadensis) & (0.09) & $(0.22)$ & $(0.22)$ & $(0.27)$ & $(0.24)$ & $(0.23)$ & $(0.10)$ & (0.11) & $(0.10)$ & & \\
\hline
\end{tabular}


Table 2.2. Continued.

\begin{tabular}{|c|c|c|c|c|c|c|c|c|c|c|c|}
\hline \multirow[b]{4}{*}{ Species } & \multicolumn{11}{|c|}{ Treatment } \\
\hline & \multicolumn{3}{|c|}{ Harvested } & \multicolumn{3}{|c|}{ Peripheral } & \multicolumn{3}{|c|}{ Unharvested } & \multirow{2}{*}{\multicolumn{2}{|c|}{$\begin{array}{c}\text { ANOVA } \\
\text { Results }\end{array}$}} \\
\hline & 1996 & 1997 & 1998 & 1996 & 1997 & 1998 & 1996 & 1997 & 1998 & & \\
\hline & $\begin{array}{c}\text { Mean } \\
(\mathrm{SE})\end{array}$ & $\begin{array}{c}\text { Mean } \\
(\mathrm{SE})\end{array}$ & $\begin{array}{c}\text { Mean } \\
(\mathrm{SE})\end{array}$ & $\begin{array}{c}\text { Mean } \\
(\mathrm{SE})\end{array}$ & $\begin{array}{c}\text { Mean } \\
(\mathrm{SE})\end{array}$ & $\begin{array}{c}\text { Mean } \\
(\mathrm{SE})\end{array}$ & $\begin{array}{l}\text { Mean } \\
(\mathrm{SE})\end{array}$ & $\begin{array}{c}\text { Mean } \\
(\mathrm{SE})\end{array}$ & $\begin{array}{c}\text { Mean } \\
(\mathrm{SE})\end{array}$ & $\mathrm{F}$ & $\mathrm{P}$-value \\
\hline Dark-eyed junco $^{c}$ & 0.61 & 1.05 & 0.83 & 0.62 & 0.59 & 0.35 & 0.24 & 0.45 & 0.48 & 2.68 & 0.08 \\
\hline (Junco hyemalis) & $(0.18)$ & $(0.26)$ & $(0.15)$ & $(0.18)$ & $(0.19)$ & $(0.17)$ & $(0.06)$ & $(0.11)$ & $(0.09)$ & & \\
\hline Magnolia & & & & & & & & & & & \\
\hline warbler $^{\mathrm{d}}(D$. & 0.61 & 0.67 & 0.72 & 0.31 & 0.47 & 0.88 & 0.30 & 0.20 & 0.50 & 4.43 & 0.02 \\
\hline magnolia) & $(0.14)$ & $(0.14)$ & $(0.16)$ & $(0.12)$ & $(0.17)$ & $(0.21)$ & $(0.06)$ & $(0.06)$ & $(0.11)$ & & \\
\hline Red-eyed vireo & 0.39 & 1.00 & 1.05 & 0.31 & 0.29 & 0.82 & 0.60 & 0.84 & 1.29 & 2.48 & 0.11 \\
\hline ( $V$. olivaceous) & $(0.12)$ & $(0.23)$ & $(0.17)$ & $(0.12)$ & $(0.11)$ & $(0.20)$ & $(0.09)$ & $(0.10)$ & $(0.11)$ & & \\
\hline Scarlet tanager & & & & & & & & & & & \\
\hline (Piranga & 0.11 & 0.22 & 0.05 & 0.31 & 0.18 & 0.07 & 0.20 & 0.25 & 0.29 & 1.47 & 0.24 \\
\hline olivacea) & $(0.08)$ & $(0.10)$ & $(0.06)$ & $(0.12)$ & $(0.09)$ & $(0.06)$ & $(0.05)$ & $(0.07)$ & $(0.07)$ & & \\
\hline Veery (Catharus & 0.39 & 0.44 & 0.17 & 0.19 & 0.47 & 0.47 & 0.38 & 0.43 & 0.39 & & \\
\hline fuscescens) & $(0.16)$ & $(0.14)$ & $(0.09)$ & $(0.10)$ & $(0.17)$ & $(0.12)$ & $(0.07)$ & $(0.09)$ & $(0.09)$ & 1.00 & 0.37 \\
\hline
\end{tabular}

${ }^{a}$ ANCOVA, test for treatment effects with pre-harvest abundance as a covariate.

${ }^{\mathrm{b}}$ Abundance significantly higher in harvested than unharvested areas (Scheffe's multiple comparison procedure, family alphalevel $=0.10$ ). 
cAbundance significantly higher in harvested areas than peripheral and unharvested areas (Scheffe's multiple comparison procedure, family alpha-level $=0.10$ ).

d Abundance significantly higher in peripheral areas than unharvested areas (Scheffe's multiple comparison procedure, family alphalevel $=0.10$ ). 
Table 2.3. Species for which nests were located on unharvested and pre-harvest treatment areas in 1996 and on unharvested (unhar.), peripheral (per.), and harvested (har.) areas on the Westvaco Wildlife and Ecosystem Research Forest, Randolph County, West Virginia, 1997-1998.

\begin{tabular}{|c|c|c|c|c|c|c|c|c|}
\hline \multirow[b]{3}{*}{ Species } & \multicolumn{8}{|c|}{ Number of Nests } \\
\hline & \multicolumn{2}{|c|}{1996} & \multicolumn{3}{|c|}{1997} & \multicolumn{3}{|c|}{1998} \\
\hline & Unhar. & Pre-har. & Unhar. & Per. & Har. & Unhar. & Per. & Har. \\
\hline \multicolumn{9}{|l|}{ Ground Nesters: } \\
\hline$\overline{\text { Canada warbler (Wilsonia canadensis) }}$ & 1 & 0 & 1 & 0 & 0 & 0 & 0 & 3 \\
\hline Dark-eyed junco (Junco hyemalis) & 2 & 4 & 5 & 2 & 3 & 2 & 4 & 6 \\
\hline Eastern towhee (Pipilo erythrophthalmus) & 3 & 1 & 0 & 0 & 0 & 2 & 0 & 0 \\
\hline Hermit thrush (Catharus guttatus) & 0 & 1 & 0 & 2 & 0 & 0 & 2 & 0 \\
\hline Ovenbird (Seiurus aurocapillus) & 0 & 0 & 0 & 1 & 0 & 0 & 1 & 0 \\
\hline Veery (C.fuscescens) & 2 & 3 & 1 & 0 & 0 & 2 & 1 & 1 \\
\hline \multicolumn{9}{|l|}{ Shrub Nesters: } \\
\hline$\overline{\text { Black-throated blue warbler (Dendroica caerulescens) }}$ & 1 & 1 & 0 & 0 & 0 & 1 & 0 & 0 \\
\hline Hooded warbler $(W$. citrina $)$ & 1 & 0 & 0 & 0 & 0 & 0 & 0 & 0 \\
\hline Indigo bunting (Passerina cyanea) & 3 & 0 & 0 & 0 & 0 & 0 & 0 & 0 \\
\hline \multicolumn{9}{|l|}{ Subcanopy Nesters: } \\
\hline American robin (Turdus migratorius) & 0 & 3 & 2 & 0 & 0 & 0 & 0 & 2 \\
\hline Blue-headed vireo (Vireo solitarius) & 0 & 0 & 4 & 0 & 0 & 1 & 2 & 2 \\
\hline Brown creeper (Certhia americana) & 0 & 1 & 0 & 0 & 0 & 0 & 0 & 0 \\
\hline Magnolia warbler (D. magnolia) & 0 & 0 & 1 & 0 & 0 & 1 & 0 & 0 \\
\hline Red-eyed vireo ( $V$. olivaceous $)$ & 0 & 0 & 1 & 0 & 0 & 0 & 0 & 0 \\
\hline Rose-breasted grosbeak & & & & & & & & \\
\hline (Pheucticus ludovicianus) & 0 & 1 & 0 & 0 & 0 & 0 & 0 & 0 \\
\hline Wood thrush (Hylocichla mustelina) & 0 & 2 & 0 & 0 & 0 & 0 & 1 & 0 \\
\hline \multicolumn{9}{|l|}{ Canopy Nesters: } \\
\hline 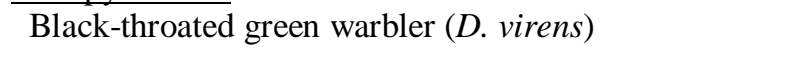 & 0 & 1 & 0 & 0 & 0 & 1 & 2 & 0 \\
\hline \multicolumn{9}{|l|}{ Cavity Nesters: } \\
\hline Black-capped chickadee (Poecile atricapillus) & 0 & 1 & 0 & 0 & 0 & 0 & 0 & 0 \\
\hline Winter wren (Troglodytes troglodytes) & 0 & 1 & 0 & 0 & 0 & 0 & 0 & 0 \\
\hline
\end{tabular}


Table 2.4. Mayfield daily nest survival estimates, exposure days, and total survival for nesting guilds and for total nests in unharvested, harvested, and peripheral areas on the Westvaco Wildlife and Ecosystem Research Forest, Randolph County, West Virgnia,1996-1998. Daily and total survival were calculated as the product of survival during the incubating and brooding periods.

\begin{tabular}{|c|c|c|c|c|c|c|c|c|c|}
\hline \multirow{4}{*}{$\begin{array}{l}\text { Guild/ } \\
\text { Treatment }\end{array}$} & \multicolumn{9}{|c|}{ Year } \\
\hline & \multicolumn{3}{|c|}{1996 (Pre-harvest) } & \multicolumn{3}{|c|}{1997} & \multicolumn{3}{|c|}{1998} \\
\hline & Exp. & Daily & Total & Exp. & Daily & Total & Exp. & Daily & Total \\
\hline & Days & Surv. & Surv. & Days & Surv. & Surv. & Days & Surv. & Surv. \\
\hline \multicolumn{10}{|l|}{ Total: } \\
\hline Unharvested & 137.5 & 0.956 & 0.372 & 128.5 & 0.926 & 0.142 & 114.5 & 0.941 & 0.284 \\
\hline Harvested & 174.5 & 0.926 & 0.149 & 47.5 & 0.980 & 0.650 & 146.0 & 0.967 & 0.503 \\
\hline Peripheral $^{\mathrm{a}}$ & -- & -- & -- & 47.0 & 0.959 & 0.357 & 139.0 & 0.972 & 0.464 \\
\hline \multicolumn{10}{|l|}{ Ground: } \\
\hline Unharvested & 92.5 & 0.958 & 0.391 & 60.5 & 0.934 & 0.171 & 66.0 & 0.939 & 0.270 \\
\hline Harvested & 99.0 & 0.933 & 0.191 & 47.5 & 0.981 & 0.650 & 77.5 & 0.950 & 0.431 \\
\hline Peripheral & -- & -- & -- & 47.0 & 0.959 & 0.357 & 81.0 & 0.976 & 0.476 \\
\hline \multicolumn{10}{|l|}{ Shrub: } \\
\hline Unharvested & 45.0 & 0.958 & 0.348 & -- & --- & --- & 4.0 & 0.750 & 0.012 \\
\hline Harvested & 15.5 & 0.900 & 0.207 & -- & --- & --- & -- & -- & -- \\
\hline Peripheral & -- & -- & -- & -- & --- & --- & -- & -- & -- \\
\hline \multicolumn{10}{|l|}{ Subcanopy: } \\
\hline Unharvested & -- & -- & -- & 40.5 & 0.929 & 0.117 & 16.5 & 0.951 & 0.214 \\
\hline Harvested & 40.5 & 0.902 & 0.047 & -- & --- & --- & 41.5 & 0.976 & 0.506 \\
\hline Peripheral & -- & -- & -- & -- & --- & --- & 10.5 & 0.905 & 0.319 \\
\hline \multicolumn{10}{|l|}{ Canopy: } \\
\hline Unharvested & -- & -- & -- & 27.5 & 0.905 & 0.135 & 28.0 & 0.964 & 1.000 \\
\hline Harvested & -- & -- & -- & -- & --- & --- & 27.0 & 1.000 & 1.000 \\
\hline Peripheral & -- & -- & -- & -- & --- & --- & 38.5 & 0.974 & 0.451 \\
\hline
\end{tabular}

${ }^{a}$ Harvested and peripheral areas were not separated in 1996 because they were still unharvested forest. 
Table 2.5. Comparison of habitat variables in stands harvested using the diameter-limit method and in unharvested stands on the Westvaco Wildlife and Ecosystem Research Forest, Randolph County, West Virginia in 1998. Means are for untransformed data.

\begin{tabular}{|c|c|c|c|c|c|c|}
\hline \multirow[b]{3}{*}{ Variable } & \multicolumn{4}{|c|}{ Treatment } & \multirow[b]{3}{*}{$\mathrm{F}$} & \multirow[b]{3}{*}{ P-value } \\
\hline & \multicolumn{2}{|c|}{ Harvested } & \multicolumn{2}{|c|}{ Unharvested } & & \\
\hline & Mean & SE & Mean & SE & & \\
\hline \multicolumn{7}{|c|}{ Woody spp. (no./ha): } \\
\hline$>8-14.9 \mathrm{~cm}$ & 225.0 & 34.8 & 177.2 & 14.9 & 4.08 & 0.06 \\
\hline$>15-22.9 \mathrm{~cm}$ & 133.1 & 19.0 & 117.0 & 11.4 & 0.03 & 0.87 \\
\hline$>23-29.9 \mathrm{~cm}$ & 93.1 & 9.0 & 85.0 & 10.9 & 0.63 & 0.44 \\
\hline$>30-37.9 \mathrm{~cm}$ & 60.0 & 8.7 & 50.7 & 7.8 & 0.01 & 0.92 \\
\hline$>38 \mathrm{~cm}$ & 50.6 & 9.6 & 69.7 & 5.5 & 0.50 & 0.49 \\
\hline Snags & 109.3 & 24.1 & 35.2 & 12.0 & 17.91 & $<0.01$ \\
\hline Shrubs & 2181.2 & 941.4 & 5381.2 & 2024.6 & 2.29 & 0.15 \\
\hline Saplings & 331.2 & 221.7 & 820.0 & 164.2 & 4.70 & 0.05 \\
\hline Poles & 375.0 & 123.3 & 548.7 & 77.2 & 1.90 & 0.19 \\
\hline \multicolumn{7}{|c|}{ Cover variables $(\%)$ : } \\
\hline$\geq 0.5-3 \mathrm{~m}$ & 28.5 & 13.5 & 32.6 & 16.1 & 0.35 & 0.56 \\
\hline$>3-6 \mathrm{~m}$ & 38.9 & 10.6 & 42.3 & 14.0 & 0.41 & 0.53 \\
\hline$>6-12 \mathrm{~m}$ & 34.9 & 16.2 & 43.2 & 13.0 & 0.84 & 0.37 \\
\hline$>12-18 \mathrm{~m}$ & 36.2 & 16.1 & 44.1 & 15.6 & 0.60 & 0.45 \\
\hline$>18-24 \mathrm{~m}$ & 53.7 & 24.8 & 51.1 & 15.2 & 0.84 & 0.38 \\
\hline$>24 \mathrm{~m}$ & 43.1 & 26.0 & 42.9 & 19.2 & 0.57 & 0.46 \\
\hline Green & 24.6 & 19.1 & 30.6 & 14.2 & 1.72 & 0.21 \\
\hline Litter & 49.9 & 22.2 & 54.1 & 16.3 & 0.03 & 0.86 \\
\hline Down Wood & 11.4 & 8.1 & 5.3 & 2.7 & 3.35 & 0.09 \\
\hline Bareground/rock & 9.6 & 6.6 & 9.2 & 7.9 & 0.03 & 0.87 \\
\hline
\end{tabular}


Table 2.6. Comparison of species-specific responses to partial harvesting methods in different regions of the United States.

\begin{tabular}{|c|c|c|c|c|c|}
\hline \multirow[b]{3}{*}{ Location } & \multicolumn{5}{|c|}{ Study } \\
\hline & $\begin{array}{l}\text { Medin \& } \\
\text { Booth }\end{array}$ & $\begin{array}{l}\text { Lent \& } \\
\text { Capen }^{\mathrm{a}}\end{array}$ & $\begin{array}{l}\text { Annand \& } \\
\text { Thompson }\end{array}$ & $\begin{array}{l}\text { Chambers } \\
\text { et al. }\end{array}$ & $\begin{array}{l}\text { This } \\
\text { Study }\end{array}$ \\
\hline & Idaho & Vermont & Missouri & Oregon & $\begin{array}{l}\text { West } \\
\text { Virginia }\end{array}$ \\
\hline Harvest Type & $\begin{array}{l}\text { Single- } \\
\text { tree }\end{array}$ & $\begin{array}{l}\text { Clearcut/ } \\
\text { group } \\
\text { selection }\end{array}$ & $\begin{array}{l}\text { Group/ } \\
\text { single-tree }\end{array}$ & $\begin{array}{l}\text { Group } \\
\text { selection }\end{array}$ & $\begin{array}{l}\text { Diameter- } \\
\text { limit } \\
(\geq 45.7-\mathrm{cm})\end{array}$ \\
\hline Species & & & Response $^{\mathrm{b}}$ & & \\
\hline Blue-headed vireo & ns & & & & ns \\
\hline Dark-eyed junco & ns & ns & & ns & + \\
\hline Magnolia warbler & & + & & & ns \\
\hline Red-eyed vireo & & ns & ns & & ns \\
\hline Scarlet tanager & & ns & ns & & ns \\
\hline Veery & & ns & & & ns \\
\hline
\end{tabular}

${ }^{a}$ Direct comparison was difficult because of methodology differences. Most species were found in both disturbed and undisturbed habitat.

b 'ns' $[$ no significant response to harvest, ' + ' = positive response. Blank cells indicate that species were not found in all studies. 


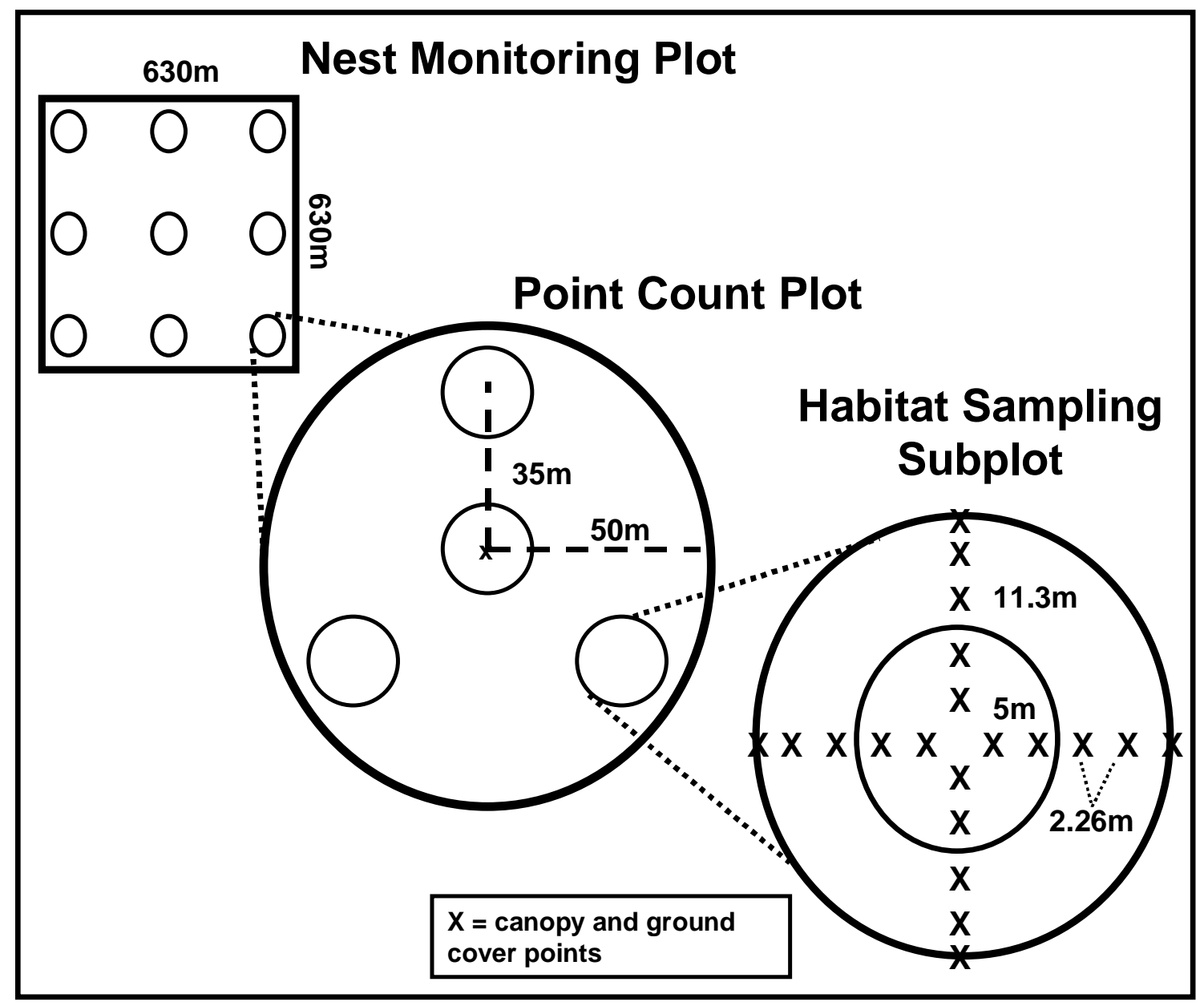

Figure 2.1. Layout of nest searching plots, point count plots, and habitat sampling subplots on the Westvaco Wildife and Ecosystem Research Forest, Randolph County, West Virginia, 1996-1998. 


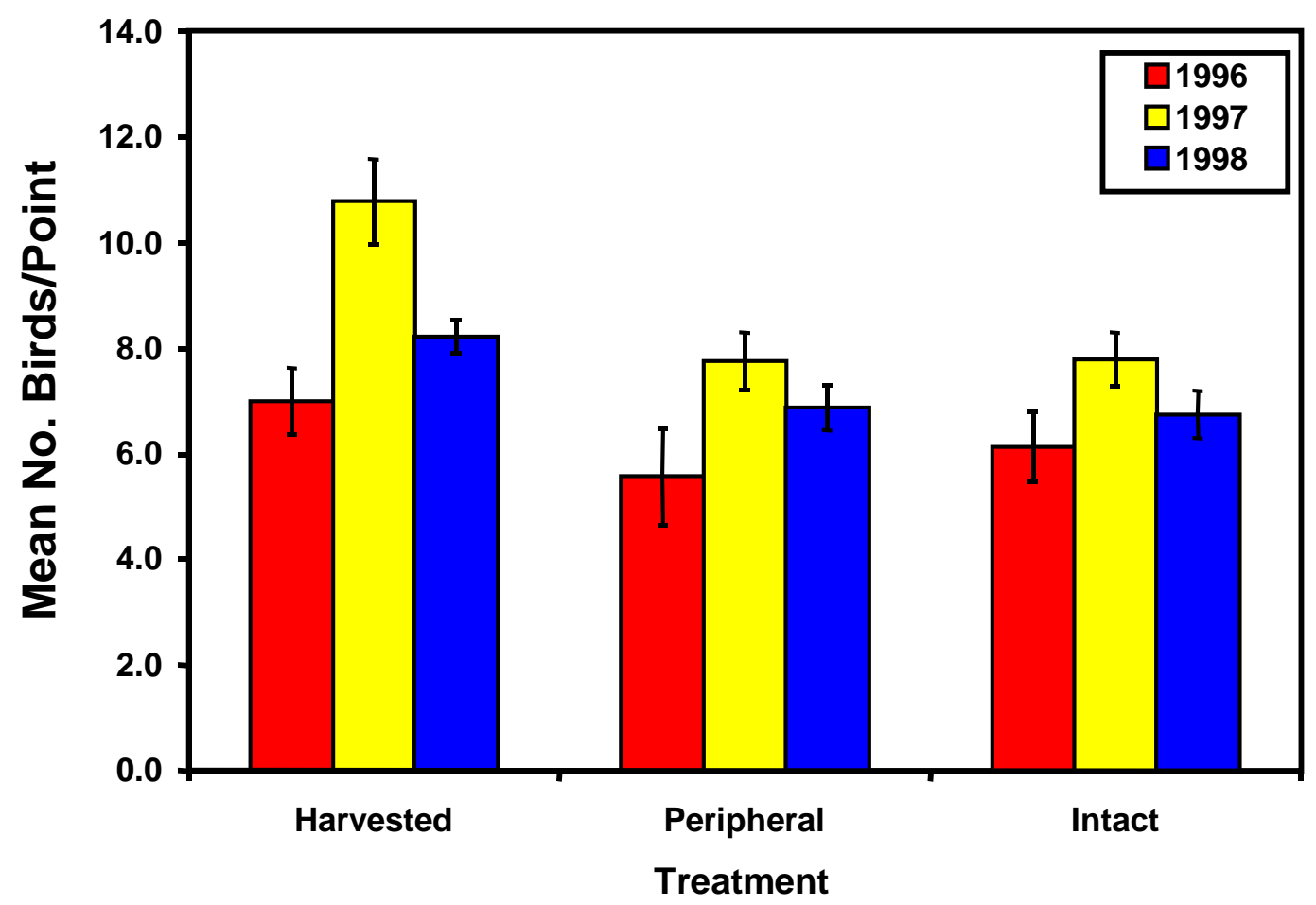

Figure 2.2. Mean bird abundance in harvested, peripheral, and unharvested stands on the Westvaco Wildife and Ecosystem Research Forest, Randolph County, West Virginia, 1997-1998. Error bars represent \pm 1 standard error. Means are for untransformed data. 


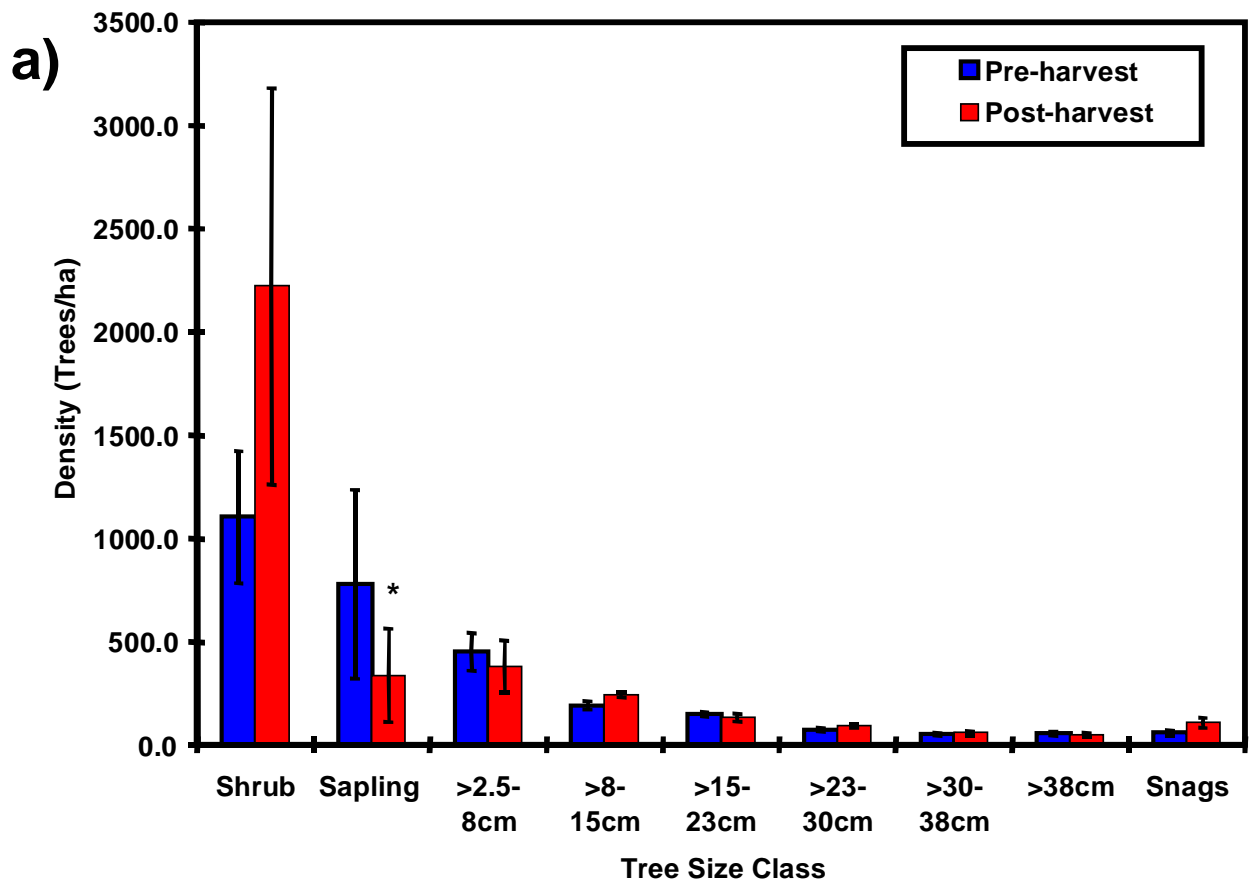

b)

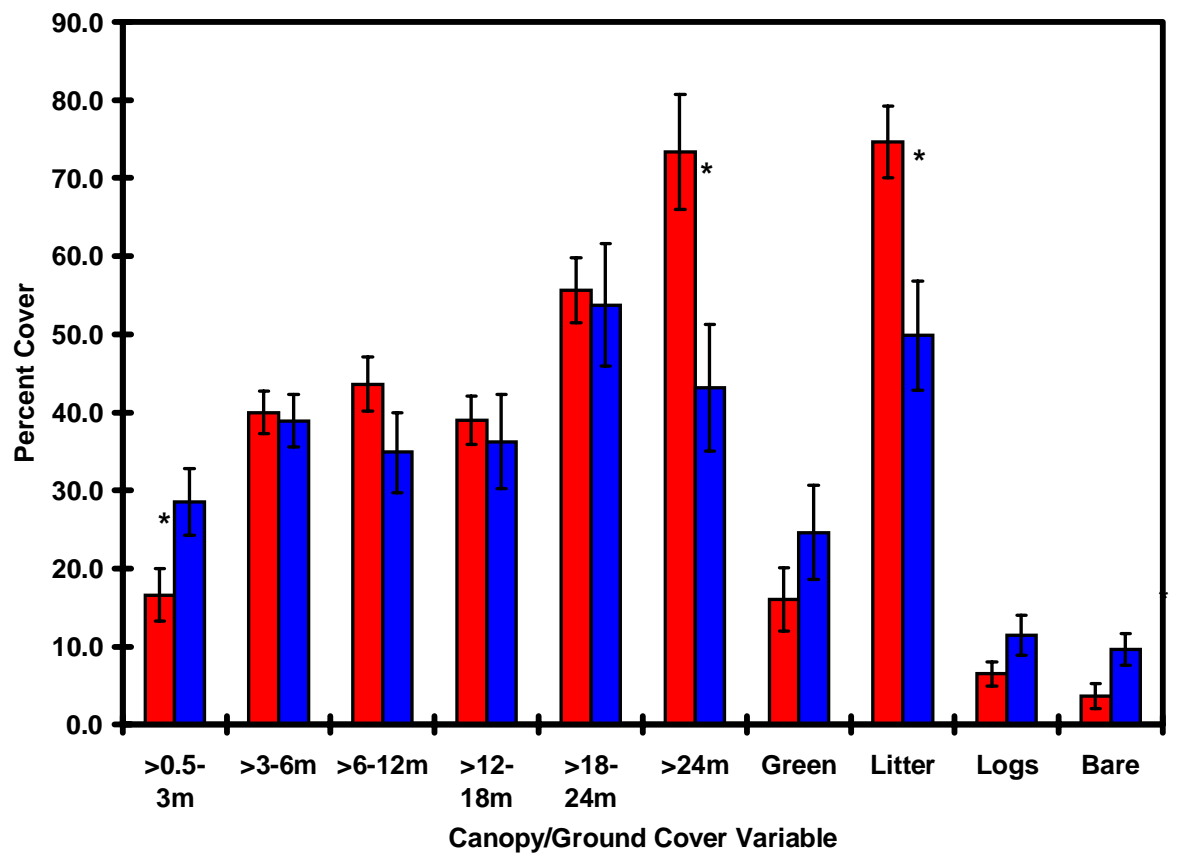

Figure 2.3. a) Mean density of woody vegetation in nine size classes and b) percent cover in six canopy layer classes and four ground cover classes before (1996) and after diameter-limit harvesting (1998) on the Westvaco Wildlife and Ecosystem Research Forest, Randolph County, West Virginia. Error bars represent \pm 1 standard error. Bars with a "*' differ at $P \leq 0.10$. Means are for untransformed data. 
CHAPTER 3

SHORT-TERM EFFECTS OF TWO-AGE HARVESTING ON SONGBIRD ABUNDANCE AND NESTING SUCCESS 
Abstract. --Two-age harvesting is a timber harvest technique where a predetermined amount of basal area is left standing to grow for the next rotation. The resulting stand is comprised of two distinct age classes. This technique is thought to be more aesthetically appealing to forest visitors than clearcutting. Previous research on 9-15 year old two-age harvests in the Appalachians indicate that some songbird species are negatively affected by this type of removal, whereas others are positively affected. The objective of this study was to determine how twoage harvesting immediately (within 1-2 years) affects songbird species. I examined the effects of this technique on songbird populations on the Westvaco Wildlife and Ecosystem Research Forest (WWERF), Randolph County, West Virginia. I examined songbird abundances throughout the forest and nesting success on four 40 ha plots in 1996. In 1997, two plots were partially harvested using the two-age method. Abundance and nesting success again were monitored in 1997 and 1998. The abundance of most songbird species present prior to harvest changed little after the timber removal. Two species, the Black-throated Green Warbler (Dendroica virens) and Red-eyed Vireo (Vireo olivaceus), were more abundant in unharvested areas than harvested stands. Interior-edge species, Blue-headed Vireos (V. solitarius), and Scarlet Tanagers (Piranga olivacea) had higher abundance in harvested areas. Pre-harvesting nest survival was $18.7 \%$ on treatment plots and $22.9 \%$ on unharvested control plots. Overall nest survival after harvesting was $42.4 \%, 16.8 \%$, and $29.0 \%$ in harvested stands, stands adjacent to harvests, and unharvested stands (>480 m from harvests), respectively. Most size classes of trees, except trees $>38 \mathrm{~cm}$, decreased in density after the harvest. Understory stem densities did not differ from prior to post-harvesting, but several measures of canopy cover in six different layer classes decreased. Unharvested stands had higher tree densities, greater canopy cover, and more vertical structure than harvested stands. The percentages of green ground cover and woody debris cover were higher in harvested stands than unharvested stands. Two-age harvesting does not appear to affect negatively most songbird species. This technique could be used in conjunction with other silvicultural methods to maintain healthy and diverse populations of songbird species in extensively forested regions. 
Two-age harvesting, or "deferment harvesting," is a timber removal method that leaves some overstory in the stand and is thought to be more attractive to forest visitors than clearcutting (Smith et al. 1989). In a two-age harvest, a predetermined amount of basal area is left standing to grow (deferred) for an entire rotation (Smith et al. 1989). The resulting stand is comprised of two different age classes, a higher vertical complexity, and more canopy cover than a clearcut. This type of partial harvesting allows landowners to retain the aesthetic value of standing timber (Miller 1993).

On the Monongahela National Forest in West Virginia, two-age stands had higher densities of some songbird species than unharvested stands 9-15 years after the harvesting event (Wood et al. 1998). However, overall abundance of forest-interior species was higher in unharvested stands whereas abundances of interior-edge and edge species were higher in two-age stands. Nest survival was higher in unharvested stands than in two-age stands or stands peripheral to two-age harvests (Wood et al. 1998). Songbird densities were found to be higher in two-age harvests 1-2 years after harvesting than in unharvested stands on the Daniel Boone National Forest in eastern Kentucky, but no estimate of nesting success between harvested and unharvested sites was determined (Baker and Lacki 1997).

The immediate post-harvesting impacts of two-age cuts on songbird species have not been examined in West Virginia. Most forest land in West Virginia is owned privately owned, and much of this property has been disturbed by forest clearing, strip mining, and other disturbances. Thus, studies on private and industrial forest lands are needed to determine the overall impact of forestry practices on breeding birds. West Virginia is approximately $76 \%$ forested, and recent surveys suggest that $35 \%$ of non-industrial private landowners plan to harvest their forests (Birch et. al 1992). Several forest songbird species are declining in the Northeast but appear to be stable in West Virginia (Rosenberg and Wells 1995). It will be important to determine how different harvesting techniques will influence songbird abundance before such large-scale harvesting occurs. However, bird density alone can be a misleading indicator of songbird habitat selection because subdominant individuals might be forced into poorer-quality habitat where reproductive success is lower (Van Horne 1983, Vickery et al. 1992). Accordingly, songbird reproductive rates as well as abundance should be examined in studies of habitat selection. 
The primary objectives of this research were: 1) To compare songbird abundance and nesting success among two-age harvested areas, unharvested areas, and areas peripheral to harvests; 2) to examine how habitat structure is altered after harvesting, and 3) to examine differences in habitat structure between harvested and unharvested areas. Timber harvests in forested landscapes may act as fragmentation events to breeding songbirds (Hagan et al. 1996). They found that the abundance of some species increased in stands adjacent to harvested areas, suggesting that birds previously holding territories in the harvest area moved to the closest available habitat. Thus bird density increased in adjacent stands. Therefore, I examined songbird abundance in stands peripheral to harvests separately from unharvested stands farther away from the harvests.

\section{STUDY SITE}

The study area was the 3360-ha Westvaco Wildlife and Ecosystem Research Forest (WWERF) in Randolph, County, West Virginia County. Westvaco Corporation established the WWERF in 1994 to serve as an area for the study of industrial forestry impacts on Appalachian ecosystems. Mature forests on the WWERF are second growth stands established by natural regeneration following wide-scale logging during the 1900-1920's (Clarkson 1993). The WWERF currently is undergoing a 10-year harvest schedule comprised of diameter-limit harvests for sawtimber production, silvicultural clearcutting (seed tree harvests) for stand regeneration, and two-aged regeneration harvests. Seventy-three percent of the forest was in the 60-79 year old age class at study initiation. Elevations range from 740 to $1200 \mathrm{~m}$. Steep side slopes with broad, plateau-like ridgetops and narrow valleys with small, high-gradient streams characterize the topography. Average annual precipitation exceeds $155 \mathrm{~cm}$, and the climate is cool and moist (Strausbaugh and Core 1977).

The forest is an Allegheny and northern hardwood type. Dominant species include red maple (Acer rubra), sugar maple (A. saccharum), American beech (Fagus grandifolia), black cherry (Prunus serotina), northern red oak (Quercus rubra) and yellow birch (Betula

alligheniensis). A few small coves are found in lower elevations and are predominantly tuliptree (Liriodendron tulipifera), American basswood, and northern red oak. Eastern hemlock (Tsuga canadensis) and red spruce (Picea rubens) occur on exposed high elevation ridges and along sheltered riparian areas. Common subcanopy and understory woody vegetation include witch 
hazel (Hamamelis virginiana), striped maple (A. pensylvanicum.), and rhododendron

(Rhododendrom maximum). Plant nomenclature follows Strausbaugh and Core (1977).

Several stands were partially harvested in the recent past and have a dense shrub layer of greenbrier (Smilax rotundifolia). In addition, the forest is transected by old logging roads and skidder trails, most of which have been re-vegetated by grasses and/or ferns and are partially to completely covered by the surrounding canopy trees. The white-tailed deer (Odocoileus virginianus) population on the forest is high, and deer have overbrowsed the understory (pers. obs.). Many stands lack a significant shrub/subcanopy layer and have virtually no herbaceous ground cover. Two stands were harvested using the two-age technique in the past 10 years and have been over-browsed by deer, and consequently they have a dense layer of hay-scented fern (Dennstaedtia punctiloba) and little hardwood regeneration.

\section{METHODS}

Nest Searching Plots.-- Four 39.7ha plots (630m x 630m) were established on the WWERF in 1996 and searched for songbird nests (Fig. 3.1). Vinyl flagging was placed approximately every 60m as an aid in relocating nests. Between August 1996 and April 1997, approximately onethird of two plots were harvested. Only one-third of each plot was cut, so I could also examine songbird nest success in stands peripheral to harvests. The other two plots were not harvested and were left as controls. In harvested stands, residual trees were spaced relatively uniform throughout the stand, but some trees were clumped along spring seeps and intermittent streams. Basal area before harvesting averaged $29.5 \mathrm{~m}^{2} / \mathrm{ha}$ and $9.5 \mathrm{~m}^{2} / \mathrm{ha}$ after. The number of residual trees left after harvest averaged 168.7 trees/ha (tree $>8.0 \mathrm{~cm})$.

Plots were visited every 2-3 days by observers trained in nest searching and monitoring techniques (Martin and Guepel 1993). Nests were checked every 3-4 days to determine clutch size, number of nestlings, and nest fate. Nests that fledged at least one young were considered successful. High nests were checked with a mirror attached to a pole. Monitoring methods were based on the Breeding Bird Research Database (BBIRD) protocols (Martin et al. 1997).

Daily nest survival and total nesting period survival for all species, for all species combined, and for nesting guilds were calculated using the Mayfield method (Mayfield 1961, 1975, Johnson 1979). Daily nest survival (DS) for the incubation and brood periods were calculated by: 


$$
\mathrm{DS}_{\text {period }}=(1-\text { number of failed nests /exposure days }) \text {. }
$$

Nest survival (S) for the incubation and brood periods were calculated by:

$$
\mathrm{S}_{\text {period }}=(\text { daily nest survival })^{\text {nest days }}
$$

The product of $S_{\text {incubation }}$ and $S_{\text {brood }}$ was used as the estimate of total nest survival for the entire nesting cycle (Mayfield 1961). The averages of the typical number of incubating days and of brooding days of all species found in each treatment were taken as the typical nest days when calculating nest survival for all species combined. The typical nest days for guilds was determined by taking the average of all species for which we found nests in each guild in each treatment. Survival during the egg-laying stage was excluded because of small sample sizes for this period, and exposure days were calculated from the first incubation day. Nesting guilds were based on the general location of species' nests: ground, shrub, subcanopy, canopy, and cavity. Species were designated to a particular guild based on Ehrlich et. al (1988) and from personal observations of nests on the WWERF. Survival rates were not compared statistically due to small sample sizes.

Songbird Abundance.-- I determined songbird abundance using standardized $50 \mathrm{~m}$ radius point counts (Ralph et. al 1993). All birds seen or heard during a 10 min count were recorded. Counts were not conducted if it was raining or if wind interfered with hearing. One hundred forty-six point count stations were located throughout the forest on a $241 \mathrm{~m}$ x $241 \mathrm{~m}$ grid system established by Westvaco. All points $<1000 \mathrm{~m}$ from any disturbance other than two-age harvests were excluded from analyses, thus only 72 points were used to examine effects of two-age harvesting on songbird abundance. Point count stations were found to be spatially independent (Weakland and Wood, unpub. rept). All points were unharvested in 1996. In 1997-98 seven points in four stands were within harvests, 13 points in ten stands were peripheral to a harvest, and 52 points in 42 stands were left unharvested. Points were classified as peripheral if they were $>50 \mathrm{~m}$ from but $<480 \mathrm{~m}$ from a harvest. Points were spaced either $241 \mathrm{~m}$ or $482 \mathrm{~m}$ apart and were surveyed twice each year. The maximum number of birds between these two counts was used in analyses. Two observers who had previous songbird identification experience conducted all counts.

Songbird abundance was analyzed by placing birds into habitat groups (edge, interioredge, and forest-interior) and into nesting guilds (ground, shrub, subcanopy, canopy, and cavity). Birds were placed into these guilds and groups based on Whitcomb et. al (1981), Ehrlich et. al 
(1988) and from personal observation of species on the WWERF. I also analyzed the total abundance of birds and the abundance of individual species that were detected at $\geq 20 \%$ of point counts (R. Harner, pers. comm.). Species nomenclature follows the American Ornithologists' Union checklist of North American birds (American Ornithologists' Union 1999).

I calculated a vertical structure index for each point count. Canopy cover in six layer classes was determined using the site-tube method of James and Shugart (1970) at each point count. The six layer classes were: $\geq 0.5-3 \mathrm{~m},>3-6 \mathrm{~m},>6-12 \mathrm{~m},>12-18 \mathrm{~m},>18-24 \mathrm{~m}$, and $>24 \mathrm{~m}$. These classes approximately describe the presence of shrubs, saplings, pole-size trees, and subdominant trees in the understory and of codominant and dominant trees in the overstory. To determine canopy cover, I established four 0.04 ha subplots within the $50 \mathrm{~m}$ radius of each point count circle: one centered on the point count, and the other three $35 \mathrm{~m}$ away at $0^{\circ}, 120^{\circ}$, and $240^{\circ}$ degrees (Fig. 3.1, Martin et. al 1997). I recorded 20 presence/absence readings along two 22.6m transects that bisected the subplot (Fig. 3.1). The average canopy cover of these four subplots was used to calculate a vertical structure index (Nichols 1996). These measurements were taken in 1996 before harvest and again in 1998.

The index of vertical structure was included as a covariate in statistical models for individual species when there was a significant correlation between abundance and vertical structure, and when the relationship between abundance and vertical structure was homogenous among treatments (Cody and Smith 1997). Vertical structure was included in the models because dense vegetation can attenuate bird songs and change detectability of some species; for example, songbird detection probabilities may be much different in clearcuts than mature forests (Pendleton 1995). I examined the least-squared means of songbird abundance among the three treatments using two-factor analysis of covariance (ANCOVA). Treatment and year were main effects, with stand nested within treatment. Pre-harvest abundance and vertical structure were covariates. I tested for treatment effects and treatment by year interactions. If a treatment effect was detected, a subsequent Scheffe's multiple comparison test was conducted to determine differences between pairs of the least-squared treatment means (Neter et al. 1996). An important assumption in ANCOVA is that the slope of the regression line of the covariate by independent variable must be the same for all factor levels of the independent variable (Cody and Smith 1991). Thus, I tested for homogeneity of slopes before running the ANCOVA for each species and guild. If a species or guild showed a significant pre-harvest abundance by treatment 
interaction I did not use ANCOVA (Cody and Smith 1991). For these species, I used analysis of variance (ANOVA) to examine the effect of treatment on post-harvest abundance (Neter et al. 1996). Abundance estimates were transformed using the $\log _{10}($ variable+1) transformation to help normalize residuals (Nur et al., 1999).

Habitat Structure.-- I measured habitat variables on each 0.04-ha subplot in point count plots in 1996 and 1998. All trees were counted within the subplot and placed into 1 of 5 size classes: $>8-14.9 \mathrm{~cm},>15-22.9 \mathrm{~cm},>23-29.9 \mathrm{~cm},>30-37.9 \mathrm{~cm}$, and $>38 \mathrm{~cm}$ diameter at breast height (dbh)(Martin et al. 1997). All shrubs, saplings, and poles were counted within a 5.0m radius circle. Shrubs were all woody vegetation $<0.5 \mathrm{~m}$ and less $<1.4 \mathrm{~m}$ tall. Saplings were trees $\geq 1.4 \mathrm{~m}$ tall and $<2.5 \mathrm{~cm}$ at $10 \mathrm{~cm}$ above the ground. Poles were all trees $\geq 2.5-7.9 \mathrm{~cm} \mathrm{dbh}$. I also examined canopy cover and ground cover. Ground cover was determined in the same manner as canopy cover. Ground cover classes were: green vegetation (herbaceous vegetation, ferns, moss, grasses, and shrubs $<0.5 \mathrm{~m}$ tall), leaf litter, woody debris, and bareground/rock. I analyzed changes in habitat structure before and after harvesting using paired Wilcoxon rank sum tests (Hollander and Wolfe 1973), and I used nested ANOVA (stand nested within treatment) to examine differences between harvested and unharvested points. Percentage data were subjected to arcsine-square root transformations; tree and understory stem densities were converted using the transformation $\log _{10}($ variable+1) (Dowdy and Wearden 1991).

Proc GLM (SAS ${ }^{\circledR}$ Institute 1991) was used to conduct all ANCOVA and ANOVA tests. $\mathrm{JMP}^{\circledR}$ software was used to conduct Wilcoxon rank sum tests $\left(\mathrm{SAS}^{\circledR}\right.$ Institute 1995). Differences were considered significant at $\mathrm{P} \leq 0.10$ for all main-effects models. The conservative $\mathrm{P}$-value of 0.10 was chosen in order to minimize the probability of a Type II error, which may be more important in ecological situations than minimizing a Type I error (Askins et al. 1990, Johnson 1999). Error rates were controlled for all treatment comparisons for a bird species, but not experiment-wide for all bird species. Scheffe' multiple comparison tests were considered significant at a family alpha-level of 0.10 .

\section{RESULTS}

Nest Survival.--One hundred and three nests of 19 species were found and monitored throughout the course of this study (Table 3.1). Thirty-six nests were located in 1996 before harvesting, 30 on unharvested control plots and 6 on pre-harvest treatment plots (Table 3.1). In 1997, 5 nests 
were located within harvested areas, 12 in peripheral areas, and 15 in unharvested forest. In 1998, 7 nests were found within harvested areas, 7 in peripheral areas, and 21 in unharvested forest.

Total nest survival in 1996 was $25.0 \%$ on unharvested control plots and $22.9 \%$ on preharvested plots (Table 3.2). Nest survival was higher in harvested areas than in peripheral areas or unharvested areas in both 1997 and 1998. Survival in peripheral areas was lower than unharvested area in both years following harvest. Survival in harvested areas increased greatly from 1996 (pre-harvest) to 1997 and 1998 (Table 3.2). However, nest survival was similar among all three years in unharvested areas, indicating that annual variability probably was not a factor in survival changes in harvested and peripheral areas.

Eight-nine percent of nests that failed were lost to predation, $5 \%$ were lost to abandonment, and 5\% were lost because ofunknown factors on unharvested control plots. In harvested and peripheral areas, $89 \%$ of nests that failed were lost to predation and $11 \%$ failed because of unknown factors. Only one nest, which was in a peripheral area in 1997, was parasitized by a Brown-headed Cowbird (Molothrus ater). Songbird Abundance. -- The Dark-eyed Junco (Junco hyemalis, $\mathrm{R}=-0.37, \mathrm{P}=0.01$ ), Blue-headed Vireo (Vireo solitarius, $\mathrm{R}=-0.32 \mathrm{P}=0.01$ ), Canada Warbler (Wilsonia canadensis, $\mathrm{R}=-0.30$, $\mathrm{P}=0.01)$, Red-eyed Vireo (V. olivaceous, $\mathrm{R}=0.23, \mathrm{P}=0.05)$, ground nesting guild $(\mathrm{R}=-0.40$, $\mathrm{P}=0.01)$, and total abundance $(\mathrm{R}=-0.29, \mathrm{P}=0.01)$ were significantly correlationed with vertical structure, indicating that detectability is variable among stands. Thus, vertical structure was included as a covariate in ANCOVA models for these species.

Abundance of the Black-throated Green Warbler (Dendroica virens), the Blue-headed Vireo, and the Scarlet Tanager (Piranga olivacea) differed among treatments (Table 3.3). The Black-throated Green Warbler was more abundant in unharvested than peripheral and two-age areas, and the Blue-headed Vireo and Scarlet Tanager were more abundant in harvested areas than in peripheral or unharvested areas. Abundances of the Red-eyed Vireo had a significant treatment by year interaction $(\mathrm{F}=2.70, \mathrm{P}=0.08)$. This species was more abundant at unharvested points than two-age points in 1998, but did not differ among treatments in 1997 (Table 3.3).

Interior-edge species $(\mathrm{F}=5.23, \mathrm{P}=0.01)$ (Table 3.4) and total abundance $(\mathrm{F}=5.66, \mathrm{P}=0.01)$ (Fig. 3.2) exhibited significant treatment by year interactions. Interior-edge species were more abundant in harvested areas than peripheral areas in $1997(\mathrm{~F}=5.33, \mathrm{P}=0.01)$ but did not differ 
among treatments in $1998(\mathrm{~F}=1.59, \mathrm{P}=0.22)$. Total abundance was higher in harvested areas than in peripheral and unharvested areas in $1997(\mathrm{~F}=1.74, \mathrm{P}=0.08)$ but did not differ in $1998(\mathrm{~F}=1.12$, $\mathrm{P}=0.33$ ) (Fig. 3.2). For the shrub-nesting guild there was a significant interaction between treatment and pre-harvest abundance $(\mathrm{F}=3.84, \mathrm{P}=0.03)$, so an ANOVA was conducted to examine treatment effects on post-harvest abundance without pre-harvest abundance as a covariate. No treatment effects were detected for this guild (Table 3.4).

Habitat Structure.-- Stem density of trees in all size classes decreased from 1996 to 1998 at harvested points (Figure 3.3). Poles decreased $(\mathrm{W}=-10.5, \mathrm{P}=0.03)$ ); shrubs increased $(\mathrm{W}=-8.5$, $\mathrm{P}=0.09)$, and saplings $(\mathrm{W}=4.5, \mathrm{P}=0.44)$ and snags $(\mathrm{W}=-1.17, \mathrm{P}=0.28)$ did not change (Fig. 3.33.4). Canopy cover in all layer classes decreased after harvesting (Figure 3.5). The amount of litter cover was greater before harvesting $(\mathrm{W}=10.5, \mathrm{P}=0.03)$. Vertical structure also was greater before harvesting $(\mathrm{W}=10.5, \mathrm{P}=0.03)$.

Several habitat variables differed between harvested and unharvested stands in 1998 (Table 3.5). Three tree size classes (the 2 smallest and the largest) and poles were denser in unharvested stands (Table 3.5). Shrubs had higher densities in harvested stands, and snag and sapling densities did not differ between unharvested and harvested stands (Table 3.5). All of the canopy cover estimates were higher in unharvested than harvested stands (Table 3.5). Vertical structure indices were less (4.71) in harvested stands than in unharvested stands (10.67) $(\mathrm{F}=11.80, \mathrm{P}<0.01)$. Except for bareground/rock, all of the ground cover variables differed between unharvested and harvested stands. Harvested stands had higher green vegetation cover and higher woody debris cover, whereas unharvested sites had higher litter cover (Table 3.5).

\section{DISCUSSION}

Although two-age harvests appear to have significantly different vegetation structure 1and 2-years after harvesting, they did not appear to significantly affect the abundance of most songbird species. Percent canopy cover in several layer classes decreased, the amount of green vegetation ground cover increased, and the densities of several size classes of trees decreased. Differences also were observed between unharvested and harvested stands. Given these results, I expected that songbird abundances would be significantly different among treatments. However, most individual species did not differ among treatments, and the abundance of canopy-nesting and forest-interior species did not differ between harvested and unharvested sites. 
Few differences in bird abundances among harvested, peripheral, and unharvested stands were uncovered by Wood et al. (1998). Forest-interior species had higher abundances in unharvested stands than 9-15 year old two-age or peripheral stands, and interior-edge and edge species were more abundant in 9-15 year old two-age harvests than unharvested or peripheral stands. In my study, only interior-edge species differed in abundance among unharvested, peripheral and harvested stands.

One species expected to be more abundant in unharvested areas, the Scarlet Tanager, was more abundant in harvested areas than unharvested and peripheral areas in my study. This is in contrast to Wood et al. (1998) who found Scarlet Tanagers at higher densities in clearcuts and unharvested areas than in two-age harvests on the Monongahela National Forest (MNF) in West Virginia. They also observed Red-eyed Vireos, Black-throated Green Warblers, and Veerys on their study area and found that the abundance of all of these species did not differ among unharvested, peripheral, and harvested stands. Of these species, both the Red-eyed Vireo and Black-throated Green Warbler were more abundant in unharvested stands on my site. Red-eyed Vireos also were found in greater abundance in unharvested stands than in two-age harvests in Kentucky (Baker and Lacki 1997). These results indicate that the Red-eyed Vireo and potentially the Black-throated Green Warbler are negatively affected by two-age harvesting in the short-term, but these impacts are only temporary. As stand development proceeds, both of these species will return to the harvested area.

My results might vary from Wood et al. (1998) for several reasons. First, their studies were conducted between 9 and 15 years after harvesting. Thus, a significant amount of woody vegetation had regenerated on their stands as compared to my study area that was one- and twoyears post-harvest. They estimated a stem density of $8200 \mathrm{stems} / \mathrm{ha}$ of trees $<15 \mathrm{~cm}$, whereas on my study area I estimated a stem density of 1813.5 stems/ha. Canopy cover from >3-6 m and >6-12 $\mathrm{m}$ on their two-age harvest also was higher in their study than mine. The vertical structure index of the five upper canopy layers averaged 3.71 on two-age harvested stands in my study, and 6.45 on two-age stands on the MNF. Second, their study was conducted on a national forest that has been minimally disturbed for several decades, whereas the WWERF periodically has been disturbed by diameter-limit harvesting and thinning operations since the 1920's. Unharvested stands on the WWERF differ considerably from unharvested stands on the MNF. Wood et al. (1998) found cover to be $>50 \%$ for all canopy layers $>12 \mathrm{~m}$ high, whereas only one 
layer in my study, >18-24 m, was over 50\% (50.9\%). In addition, deer browsing on the WWERF is high (pers. obs.), and many unharvested stands lack significant understory and herbaceous ground cover components. The vertical structure index for the five upper canopy layers on unharvested stands on the WWERF was 8.44, which is lower than the 10.16 found by Wood et al. (1998) on unharvested stands on the MNF. Duguay et al. (in press) determined that invertebrate biomass was higher on unharvested stands, which had a higher vertical structure index, than two-age stands on the MNF; they found a positive correlation between invertebrate biomass and nest survival. Silvicultural practices have an influence on breeding bird abundance and nest survival in harvested areas (Duguay et al. (in press)). Many of the birds I examined are insectivorous, and insect abundance on the WWERF might be lower than on the MNF where there is more vertical heterogeneity.

Bird abundance among group selection, single-tree selection, clearcut, and shelterwood cuts in Missouri was examined by Annand and Thompson (1997). Two-age harvests most likely resemble the shelterwood cuts that they examined, which did not yet have the overstory removed. These shelterwood treatments left residuals of $3-4 \mathrm{~m}^{2} / \mathrm{ha}$ basal area, which is slightly lower than the $9.5 \mathrm{~m}^{2} /$ ha basal area left in my study. Total bird abundance was greater in shelterwoods than mature stands, and abundance of Red-eyed Vireos was greater in mature sites than shelterwoods. Both of these results are similar to what I found in West Virginia. However, they also found abundance of Scarlet Tanagers to be greater in mature forests than shelterwoods, which is contrary to my results. However, Scarlet Tanagers are known to tolerate different amounts of canopy closure (Crawford et al. 1981). Yahner (1993) found no differences in Scarlet Tanager abundance between clearcut and mature forests in central Pennsylvania. In addition, I observed Scarlet Tanagers nesting in the center of a two-age harvest on the WWERF as well as in unharvested areas (pers. obs.).

Few published studies have examined the effects of timber harvesting techniques on songbird nesting success. Annand and Thompson (1997) were able to examine species-specific daily survival rates in clearcuts and shelterwoods in Missouri, but were not able to compare them to unharvested stands. They found nest success rates of early successional species to vary from $18-51 \%$ in harvested areas. I found a range of $16-42 \%$ survival in two-age harvests for all species combined, including both early and late-successional species. Nest survival for just edge and interior-edge species ranged from 7-50\% in my study. However, survival rates in peripheral 
stands were much lower, only $16.8 \%$. Previous researchers suggested that predator abundance is higher at the edge or interface of 2 habitat types (Gates and Gysel 1978, Chasko and Gates 1982). Similar to my study, Hanski et al. (1996) in Minnesota, found that nest predation was higher in forested areas than in open and regenerating clearcuts. Although sample size in my study was small, my results are consistent with findings in other studies.

Wood et al. (1998) observed higher nest success rates than I observed on the WWERF, with survival rates of $39.9 \%$ in two-age harvests, $35.8 \%$ in stands peripheral to harvests, and 49.1\% in unharvested stands. Nest survival was significantly higher in the unharvested stands than in the two-age and peripheral stands, but Duguay (in press) determined that all rates were sufficiently high to consider all 3 treatments as population sources. However, it should be noted that Wood et al. (1998) found higher rates (6\%) of Brown-headed Cowbird parasitism on the MNF than I found on the WWERF (1\%), and that parasitism rates were higher in their two-age harvests and periphery stands than unharvested stands. The one nest that was parasitized in my study was located in a stand adjacent to a two-age harvest.

Female Brown-headed Cowbirds perching on residual trees within two-age harvests are likely more efficient at locating songbird nests than those perched on a tree that is the same height as the surrounding vegetation (Gates and Gysel 1978). Songbirds feeding nestling Brown-headed Cowbirds might incur more energetic costs that lower survival rates. Two-age harvests might prove to be detrimental to songbird species if they attract Brown-headed Cowbirds, regardless of nest survival rates. My sites were only recently cut, and little tree regeneration had occurred by study completion. Brown-headed Cowbirds might not begin to search for nests in these areas until more vegetation has developed.

Predation was the main cause of nest failure on the WWERF. Potential nest predators observed on the study area included Blue Jays (Cyanocitta cristata), American Crows (Corvus brachyrhynchos), Common Ravens (Corvus corax), eastern chipmunks (Tamias striatus), whitefooted mice (Peromyscus leucopus), striped skunks (Mephistis mephistis), red squirrels (Tamiasciurus hudsonicus), southern flying squirrels (Glaucomys volans), black bears (Ursus americanus), and raccoons (Procyon lotor). Higher rates of nest predation might have occurred on the WWERF than on the MNF because of the high amount of linear edge created by skidder trails and logging roads that act as travel lanes for predators. It is possible that typical movement 
patterns of predators are disrupted in harvested areas, and this might contribute to the higher nest survival rates in harvested stands.

It appears that in the short-term, two-age harvesting is not detrimental to most songbird species nesting on the WWERF. Two-age harvesting provides early successional habitat for many early successional songbird species such as Chestnut-sided Warblers, Indigo Buntings (Passerina cyanea), and Eastern Towhees (Pipilo erythrophthalmus ) (Nichols 1996). Some of these species, such as the Indigo Bunting, are experiencing significant population declines in the eastern United States, possibly due to a lack of available habitat as regional forests continue to mature (Sauer and Droege 1992). Two-age harvesting provides increased early successional habitat for these species while continuing to provide some mature canopy trees for canopy and subcanopy-nesting species. However, two songbird species, the Black-throated Green Warbler and the Red-eyed Vireo, appeared to be negatively affected by this type of harvesting. Both species had lower abundance in two-age stands in this study and in previous studies of older twoage harvests (Wood et al. 1998). Two-age harvesting also could attract Brown-headed Cowbirds to the forest in the future if cowbird feeding habitat becomes more available near the WWERF.

Effects of harvesting on songbird species might vary by geographic region, and results from West Virginia might not be applicable to other areas of a species' range. This study was confined to one industrial forest in a heavily forested region of West Virginia. Other results might be observed in areas that are less disturbed than industrial forests or in areas that are more fragmented by agriculture and urbanization.

Thompson et al. (1995) suggest that silviculturally-sound harvesting in extensively forested landscapes is compatible with conservation of songbird species. Forest managers in these regions interested in conserving populations of songbirds should employ several management methods to ensure a healthy and diverse community of avian species on their forests (Hunter 1990). Partial harvesting techniques such as single-tree and group selection provide vertical heterogeneity and habitat for forest-interior and interior-edge species. Alternatively, even-age management such as clearcutting, and two-age and shelterwood harvesting, provides habitat for edge, interior-edge species, and early successional species. Forest managers also might provide habitat for mature-forest and old growth-dependent species by not harvesting some stands, or by setting long rotation lengths (Hunter 1990). Thus, managers should use several silvicultural systems and attempt to balance age classes of stands on 
their forest to provide habitat for a variety of songbird species (Thompson et al. 1995). Two-age harvesting could be used by managers in conjunction with these other techniques to provide songbird habitat and also to appeal to the aesthetic values of the general public in their region.

\section{ACKNOWLEDGEMENTS}

This research was funded by Westvaco Corporation, Forest Resources Division, and the West Virginia Division of Natural Resources Nongame Wildlife and Natural Heritage Program. The West Virginia Cooperative Fish and Wildlife Research Unit, USGS, BRD, provided equipment and logistical support. Mark Ford, Sydney McIntyre, Sarah Clapham, and Sally Lane provided technical and field assistance. We also thank the many field technicians who assisted with nest searching, point count surveys, and habitat sampling. R. C. Whitmore, L. S. Gribko, A. F. Egan, and W. M. Ford provided helpful suggestions on earlier drafts of this manuscript.

\section{LITERATURE CITED}

AMERICAN ORNITHOLOGISTS' UNION. 1999. The American Ornithologists' Union checklist of North American birds. http://pica.wru.umt.edu/AOU/birdlist.HTML.

ANNAND, E.M. AND F.R. THOMPSON III. 1997. Forest bird response to regeneration practices in central hardwood forests. J. Wildl. Manage. 61: 159-171.

ASKINS, R.A., J.F. LYNCH, AND R. GREENBURG. 1990. Population declines in migratory birds in eastern North America. Curr. Ornith. 7: 1-57.

BAKER, M.D. AND M.J. LACKI. 1997. Short-term changes in bird communities in response to silvicultural prescriptions. For. Ecol. Manage. 96: 27-36.

BIRCH, T.W., D.A. GASNE., S.L. ARNER, AND R.H. WIDMANN. 1992. Cutting activity on West Virginia timberlands. North. J. Appl. For. 9: 146-148.

CHASKO, G. G. AND J. E. GATES. 1982. Avian habitat suitability along a transmission-line corridor in an oak-hickory forest region. Wildlife Monographs 82:1-41.

CLARKSON, R.B. 1993. Destruction of the upland forest by lumbering and fire. Pages 35-46 in S. L. Stephenson, ed., Upland forests of West Virginia. McCain Printing Co., Parsons, West Virginia.

CODY, R.P. AND J.K. SMITH. 1997. Applied statistics and the SAS programming language. Prentice Hall, Upper Saddle River, New Jersey. 445pp. 
CRAWFORD, H.S., R.G. HOOPER, AND R.W. TITTERINGTON. 1981. Songbird population rsponse to silvicultural practices in central Appalachian hardwoods. J. Wildl. Manage. 45: 680-692.

DOWDY, S. AND S. WEARDEN. 1991. Statistics for research. John Wiley and Sons. New York, New York, 629pp.

DUGUAY, J.P., P.B. WOOD, AND G.W. MILLER. In press. Effects of timber harvests on invertebrate biomass and avian nest success. Wild. Soc. Bull.

EHRLICH, P.R., D.S. DOBKIN, AND D. WHEYE. 1988. The Birder's Handbook. Simon and Schuster Inc. New York, New York. 785pp.

GATES, J. E. AND L.W. GYSEL. 1978. Avian nest dispersion and fledgling success in fieldforest ecotones. Ecology 58:871-883.

HANSKI, I.K., T.J. FENSKE, AND G.J. NIEMI. 1996. Lack of edge effect in nesting success of breeding birds in managed forest landscapes. Auk 113: 578-585.

HOLLANDER, M. AND D.A. WOLFE. 1973. Nonparametric statistical methods. John Wiley and Sons, New York, New York. 503pp.

HUNTER, M. L., JR. 1990. Wildlife, forests, and forestry: principles of managing forests for biological diversity. Prentice-Hall, Englewood Cliffs, New Jersey. 370pp.

JAMES, F.C., AND H.H. SHUGART. 1970. A quantitative method of habitat description. Aud. Field Notes 24: 727-736.

JOHNSON, D. H. 1979. Estimating nest success: the Mayfield method and an alternative. Auk 96: 651-661.

. 1999. The insignificance of statistical significance testing. J. Wildl. Manage. 63: 763-772.

MARTIN, T.E. AND G.R. GUEPEL. 1993. Nest-monitoring plots: methods for locating and monitoring success. J. Field. Ornith. 64: 507-519.

., C. PAINE, C.J. CONWAY, W. M. HOCHACHKA, P. ALLEN, and W. JENKINS. 1997. BBIRD Field Protocol. USGS, Biological Resources Division, Montana Cooperative Fish and Wildlife Research Unit, Missoula, Montana.

MAYFIELD, H.F. 1961. Nesting success calculated from exposure. Wilson Bull. 73: 255-261. 1975. Suggestions for calculating nest success. Wilson Bull. 87: 456-466. 
MILLER, G.W. 1993. Financial aspects of partial cutting practices in central Appalachian hardwoods. U.S. For. Serv. Res. Pap. NE-673.

NETER, J., M.H. KUTNER, C. J. NACHTSHEIM, AND W. WASSERMAN. 1996. Applied Linear Statistical Models. Irwin. Homewood, Illinois. 1181pp.

NICHOLS, J.V. 1996. Effects of two-age timber management and clearcutting on songbird density and reproductive success. M.S. Thesis, West Virginia University. 131pp.

NUR, N., S. L. JONES, AND G. R. R GEUPEL 1999. A statistical guide to data analysis of avian monitoring programs. U.S. Fish and Wildl. Serv. Biol. Tech. Publ. BTP-R60011999.

PENDLETON, G. W. 1995. Effects of sampling strategy, detection probability, and independence of counts on the use of point counts. Pages 131-134 in C. Ralph, J. R. Sauer, and S. Droege, eds., Monitoring bird populations by point counts. U.S. For. Serv. Gen. Tech. Rep. PSW-GTR-149.

RALPH, C.J., G.R. GUEPEL, P. PYLE, T.E. MARTIN, AND D.F. DESANTE. 1993. Handbook of field methods for monitoring landbirds. U.S. For. Serv. Gen Tech. Rept. PSW-GTR-144.

ROSENBERG, K.V. AND J.V. WELLS. 1995. Importance of geographic areas to neotropical migrant birds in the Northeast. U.S.Fish and Wildlife Serv. Rept., Region 5, Hadley, Mass.

SAS INSTITUTE INC. 1991. SAS/STAT Guide for personal computers, version 6 edition. SAS Institute, Inc. Cary, N. Carolina. 1028pp. . 1995. JMP Statistics and graphics guide, version 3.1. SAS Institute, Inc. Cary, N. Carolina. 593pp.

SAUER, J.R. AND S. DROEGE. 1992. Geographic patterns in population trends of Neotropical migrants in North America. Pages 26-42 in J.M. Hagan III, and D. W. Johnston, eds., Ecology and conservation of neotropical migrant landbirds. Smithsonian Institution Press, Washington and London.

SMITH, H.C., N.I. LAMSON, AND G.W. MILLER. 1989. An aesthetic alternative to clearcutting? J. Forestry 87:14-18.

STRAUGHSBAUGH, P.D. AND E. L. CORE. 1977. Flora of West Virginia. Seneca Books, Grantsville, West Virginia. 1079 pp. 
THOMPSON, F.R., III, J.R. PROBST, AND M.G. RAPHAEL. 1995. Impacts of silviculture: overview and management recommendations. Pages 201-219 in T. E. Martin and D. M. Finch, eds., Ecology and management of neotropical migratory birds. Oxford University Press, New York and Oxford.

VAN HORNE, B. 1983. Density as a misleading indicator of habitat quality. J. Wildl. Manage. 47: 893-901.

VICKERY, P.D., M.L. HUNTER, JR., AND J.V. WELLS. 1992. Is density an indicator of breeding success? Auk 109: 706-710.

YAHNER, R.H. 1993. Effects of long-term forest clear-cutting on wintering and breeding birds. Wilson Bull. 105: 239-255.

WHITCOMB, R.F., C.S. ROBBINS, J. F. LYNCH, B. L. KLIMKIEWICZ, AND D.

BYSTRAK. 1981. Effects of forest fragmentation on avifauna of the eastern deciduous forest. Pages 125-206 in R.L. Burgess, and D. M. Sharpe, eds., Forest island dynamics in man-dominated landscapes. Springer-Verlag, New York, New York.

WOOD, P.B., J.P. DUGUAY, AND J.V. NICHOLS. 1998. Songbird abundance, nest success, and invertebrate biomass on two-age, clearcut, and unharvested forest stands in the Monongahela National Forest, West Virginia. Final Project Rept. West Virg. Coop. Fish and Wildlife Research Unit, Biological Resources Division, USGS, Morgantown, West Virginia. 
Table 3.1. Songbird species for which nests were located on unharvested and pre-harvest treatment areas in 1996 and on unharvested (unhar.), peripheral (per.), and harvested (har.) areas in 1997-1998 on the Westvaco Wildlife and Ecosystem Research Forest, Randolph County, West Virginia , 1996-1998.

\begin{tabular}{|c|c|c|c|c|c|c|c|c|}
\hline \multirow[b]{3}{*}{ Species } & \multicolumn{8}{|c|}{ Number of Nests } \\
\hline & \multicolumn{2}{|c|}{1996} & \multicolumn{3}{|c|}{1997} & \multicolumn{3}{|c|}{1998} \\
\hline & Unhar. & Pre-har. & Unhar. & Per. & Har. & Unhar. & Per. & Har. \\
\hline \multicolumn{9}{|l|}{ Ground: } \\
\hline Black-and-white Warbler (Mniotilta varia) & 0 & 0 & 0 & 0 & 0 & 0 & 0 & 1 \\
\hline Canada Warbler (Wilsonia canadensis) & 2 & 0 & 0 & 2 & 1 & 0 & 0 & 0 \\
\hline Dark-eyed Junco (Junco hyemalis) & 0 & 0 & 1 & 1 & 3 & 0 & 1 & 2 \\
\hline Eastern Towhee (Pipilo erythrophthalmus) & 3 & 0 & 0 & 0 & 0 & 2 & 0 & 0 \\
\hline Ovenbird (Seiurus aurocapillus) & 1 & 0 & 2 & 2 & 0 & 3 & 0 & 0 \\
\hline Veery (Catharus fuscescens) & 9 & 0 & 2 & 1 & 0 & 3 & 0 & 0 \\
\hline \multicolumn{9}{|l|}{ Shrub: } \\
\hline$\overline{\text { Black-throated Blue Warbler (Dendroica caerulescens) }}$ & 0 & 2 & 4 & 0 & 0 & 4 & 1 & 0 \\
\hline Chestnut-sided Warbler (D. pensylvanica) & 0 & 0 & 0 & 0 & 1 & 0 & 0 & 0 \\
\hline Gray Catbird & 1 & 0 & 0 & 0 & 0 & 0 & 0 & 0 \\
\hline Hooded Warbler (W. citrina) & 5 & 1 & 0 & 0 & 0 & 0 & 0 & 0 \\
\hline Indigo Bunting (Passerina cyanea) & 0 & 0 & 0 & 0 & 0 & 0 & 0 & 1 \\
\hline \multicolumn{9}{|l|}{ Subcanopy: } \\
\hline Acadian Flycatcher (Empidonax viresens) & 0 & 0 & 0 & 0 & 0 & 0 & 1 & 0 \\
\hline American Robin (Turdus migratorius) & 0 & 0 & 1 & 0 & 0 & 0 & 0 & 0 \\
\hline Blue-headed Vireo (Vireo solitarius) & 0 & 1 & 0 & 0 & 0 & 2 & 0 & 1 \\
\hline Red-eyed Vireo (V. olivaceous) & 0 & 0 & 2 & 2 & 0 & 2 & 2 & 1 \\
\hline Wood Thrush (Hylocichla mustelina) & 8 & 2 & 2 & 4 & 0 & 1 & 2 & 1 \\
\hline \multicolumn{9}{|l|}{ Canopy: } \\
\hline Black-throated Green Warbler (D. virens) & 0 & 0 & 0 & 0 & 0 & 1 & 0 & 0 \\
\hline Scarlet Tanager (Piranga olivacea) & 1 & 0 & 1 & 0 & 0 & 1 & 0 & 0 \\
\hline
\end{tabular}


Table 3.1. Continued.

\begin{tabular}{|c|c|c|c|c|c|c|c|c|}
\hline \multirow[b]{3}{*}{ Species } & \multicolumn{8}{|c|}{ Number of Nests } \\
\hline & \multicolumn{2}{|c|}{1996} & \multicolumn{3}{|c|}{1997} & \multicolumn{3}{|c|}{1998} \\
\hline & Unhar. & Pre-har. & Unhar. & Per. & Har. & Unhar. & Per. & Har \\
\hline \multicolumn{9}{|l|}{ Cavity: } \\
\hline Black-capped Chickadee (Poecile atricapillus) & 0 & 0 & 0 & 0 & 0 & 1 & 0 & 0 \\
\hline Hairy Woodpecker (Picoides villosus) & 0 & 0 & 0 & 0 & 0 & 1 & 0 & 0 \\
\hline Total & 30 & 6 & 15 & 12 & 5 & 21 & 7 & 7 \\
\hline
\end{tabular}


Table 3.2. Mayfield daily nest survival estimates, exposure days, and total survival for nesting habitat groups and total nests at unharvested, harvested, and peripheral plots on the Westvaco Wildlife and Ecosystem Research Forest, Randolph County, West Virginia, 1996-98.

\begin{tabular}{|c|c|c|c|c|c|c|c|c|c|}
\hline \multirow[b]{3}{*}{$\begin{array}{l}\text { Guild/ } \\
\text { Treatment }\end{array}$} & \multicolumn{9}{|c|}{ Year } \\
\hline & \multicolumn{3}{|c|}{1996 (Pre-harvest) } & \multicolumn{3}{|c|}{1997 (Post-harvest) } & \multicolumn{3}{|c|}{1998 (Post-harvest) } \\
\hline & $\begin{array}{l}\text { Exp. } \\
\text { Days }\end{array}$ & $\begin{array}{l}\text { Daily } \\
\text { Surv. }\end{array}$ & $\begin{array}{l}\text { Total } \\
\text { Surv. }\end{array}$ & $\begin{array}{l}\text { Exp. } \\
\text { Days }\end{array}$ & $\begin{array}{l}\text { Daily } \\
\text { Surv. }\end{array}$ & $\begin{array}{l}\text { Total } \\
\text { Surv. }\end{array}$ & $\begin{array}{c}\text { Exp. } \\
\text { Days }\end{array}$ & $\begin{array}{l}\text { Daily } \\
\text { Surv. }\end{array}$ & $\begin{array}{l}\text { Total } \\
\text { Surv. }\end{array}$ \\
\hline \multicolumn{10}{|l|}{ Total: } \\
\hline Unharvested & 281.5 & 0.935 & 0.250 & 193.0 & 0.953 & 0.281 & 214.5 & 0.953 & 0.290 \\
\hline Harvested & 61.0 & 0.951 & 0.229 & 62.5 & 0.952 & 0.290 & 60.5 & 0.983 & 0.771 \\
\hline Peripheral & --- & --- & --- & 154.5 & 0.935 & 0.143 & 82.0 & 0.939 & 0.231 \\
\hline \multicolumn{10}{|l|}{ Ground: } \\
\hline Unharvested & 146.0 & 0.935 & 0.287 & 42.0 & 0.881 & 0.033 & 103.0 & 0.971 & 0.467 \\
\hline Harvested & --- & --- & --- & 41.5 & 0.952 & 0.349 & 28.5 & 1.000 & 1.000 \\
\hline Peripheral & --- & --- & --- & 78.0 & 0.929 & 0.159 & 9.0 & 0.889 & 0.063 \\
\hline \multicolumn{10}{|l|}{ Shrub: } \\
\hline$\overline{\text { Unharvested }}$ & 40.0 & 0.900 & 0.207 & 68.5 & 1.000 & 1.000 & 15.5 & 0.806 & 0.014 \\
\hline Harvested & 21.5 & 0.860 & 0.116 & 21.0 & 0.952 & 0.214 & 9.0 & 1.000 & 1.000 \\
\hline Peripheral & --- & --- & --- & --- & --- & --- & 18.5 & 1.000 & 1.000 \\
\hline \multicolumn{10}{|l|}{ Subcanopy: } \\
\hline Unharvested & 97.5 & 0.950 & 0.309 & 73.0 & 0. & 0.323 & 50.0 & 0.960 & 0.358 \\
\hline Harvested & 22.5 & 1.000 & 1.000 & --- & --- & --- & 16.5 & 0.939 & 0.398 \\
\hline Peripheral & --- & --- & --- & 76.5 & 0.935 & 0.131 & 54.5 & 0.927 & 0.167 \\
\hline \multicolumn{10}{|l|}{ Canopy: } \\
\hline Unharvested & --- & --- & --- & 9.5 & 0.895 & 0.105 & 19.0 & 0.947 & 0.603 \\
\hline Harvested & 17.0 & 1.000 & 1.000 & --- & --- & -- & 6.5 & 1.000 & 1.000 \\
\hline Peripheral & --- & --- & --- & --- & --- & -- & --- & --- & --- \\
\hline
\end{tabular}


Table 3.3. Means and standard errors (SE) for songbird abundance at harvested, peripheral, and unharvested stands one year before (1996) harvest and two-years after (1997-98) on the Westvaco Wildlife and Ecosystem Research Forest, Randolph County, West Virginia. Means are for untransformed data and are not adjusted for other terms in the model.

\begin{tabular}{|c|c|c|c|c|c|c|c|c|c|c|c|}
\hline \multirow[b]{4}{*}{ Species } & \multicolumn{11}{|c|}{ Treatment } \\
\hline & \multicolumn{3}{|c|}{ Harvested } & \multicolumn{3}{|c|}{ Peripheral } & \multicolumn{3}{|c|}{ Unharvested } & \multirow{2}{*}{\multicolumn{2}{|c|}{$\begin{array}{c}\text { ANCOVA } \\
\text { Results }^{\mathrm{a}}\end{array}$}} \\
\hline & \multirow{2}{*}{$\begin{array}{c}1996 \\
\text { Mean } \\
(\mathrm{SE})\end{array}$} & \multirow{2}{*}{$\begin{array}{c}1997 \\
\text { Mean } \\
(\mathrm{SE})\end{array}$} & \multirow{2}{*}{$\begin{array}{c}1998 \\
\text { Mean } \\
(\mathrm{SE})\end{array}$} & \multirow{2}{*}{$\begin{array}{c}1996 \\
\text { Mean } \\
(\mathrm{SE})\end{array}$} & \multirow{2}{*}{$\begin{array}{c}1997 \\
\text { Mean } \\
(\mathrm{SE})\end{array}$} & \multirow{2}{*}{$\begin{array}{c}1998 \\
\text { Mean } \\
(\mathrm{SE})\end{array}$} & \multirow{2}{*}{$\begin{array}{c}1996 \\
\text { Mean } \\
(\mathrm{SE})\end{array}$} & \multirow{2}{*}{$\begin{array}{c}1997 \\
\text { Mean } \\
(\mathrm{SE})\end{array}$} & \multirow{2}{*}{$\begin{array}{c}1998 \\
\text { Mean } \\
(\mathrm{SE})\end{array}$} & & \\
\hline & & & & & & & & & & $\mathrm{F}$ & P-value \\
\hline Black-throated & 0.17 & 0.33 & 0.17 & 0.62 & 0.23 & 0.46 & 0.36 & 0.35 & 0.30 & 0.12 & 0.89 \\
\hline Blue Warbler & $(0.17)$ & $(0.21)$ & $(0.17)$ & $(0.18)$ & $(0.12)$ & $(0.14)$ & $(0.08)$ & $(0.08)$ & $(0.07)$ & & \\
\hline Black-throated & 0.17 & 0.33 & 0.00 & 0.46 & 0.31 & 0.31 & 0.34 & 0.71 & 0.56 & 3.07 & 0.06 \\
\hline Green Warbler ${ }^{\mathrm{b}}$ & $(0.17)$ & $(0.21)$ & $(0.00)$ & $(0.18)$ & $(0.13)$ & $(0.13)$ & $(0.08)$ & $(0.12)$ & $(0.09)$ & & \\
\hline Blue-headed & 0.83 & 1.67 & 0.33 & 0.92 & 0.84 & 0.08 & 0.95 & 0.65 & 0.18 & 2.94 & 0.06 \\
\hline Vireo $^{\text {cd }}$ & $(0.17)$ & $(0.33)$ & $(0.21)$ & $(0.46)$ & $(0.19)$ & $(0.08)$ & $(0.13)$ & $(0.10)$ & $(0.06)$ & & \\
\hline \multirow[t]{2}{*}{ Canada Warbler ${ }^{\mathrm{c}}$} & 0.50 & 0.83 & 0.33 & 0.62 & 0.38 & 0.31 & 0.64 & 0.50 & 0.44 & 2.07 & 0.14 \\
\hline & $(0.22)$ & $(0.40)$ & $(0.21)$ & $(0.18)$ & $(0.14)$ & $(0.17)$ & $(0.10)$ & $(0.10)$ & $(0.10)$ & & \\
\hline Chestnut-sided & 0.00 & 0.33 & 0.50 & 0.08 & 0.00 & 0.00 & 0.18 & 0.25 & 0.16 & 0.82 & 0.45 \\
\hline Warbler & $(0.00)$ & $(0.33)$ & $(0.22)$ & $(0.08)$ & $(0.00)$ & $(0.00)$ & $(0.06)$ & $(0.07)$ & $(0.07)$ & & \\
\hline \multirow[t]{2}{*}{ Dark-eyed Junco ${ }^{c}$} & 0.00 & 0.83 & 0.17 & 0.31 & 0.15 & 0.31 & 0.14 & 0.21 & 0.20 & 0.07 & 0.93 \\
\hline & $(0.00)$ & $(0.31)$ & $(0.17)$ & $(0.13)$ & $(0.10)$ & $(0.13)$ & $(0.05)$ & $(0.07)$ & $(0.06)$ & & \\
\hline
\end{tabular}


Table 3.3. Continued.

\begin{tabular}{|c|c|c|c|c|c|c|c|c|c|c|c|}
\hline \multirow[b]{4}{*}{ Species } & \multicolumn{11}{|c|}{ Treatment } \\
\hline & \multicolumn{3}{|c|}{ Harvested } & \multicolumn{3}{|c|}{ Peripheral } & \multicolumn{3}{|c|}{ Unharvested } & \multirow{2}{*}{\multicolumn{2}{|c|}{$\begin{array}{l}\text { ANCOVA } \\
\text { Results }^{\mathrm{a}}\end{array}$}} \\
\hline & 1996 & 1997 & 1998 & 1996 & 1997 & 1998 & 1996 & 1997 & 1998 & & \\
\hline & $\begin{array}{l}\text { Mean } \\
(\mathrm{SE})\end{array}$ & $\begin{array}{l}\text { Mean } \\
(\mathrm{SE})\end{array}$ & $\begin{array}{l}\text { Mean } \\
(\mathrm{SE})\end{array}$ & $\begin{array}{l}\text { Mean } \\
(\mathrm{SE})\end{array}$ & $\begin{array}{l}\text { Mean } \\
(\mathrm{SE})\end{array}$ & $\begin{array}{l}\text { Mean } \\
(\mathrm{SE})\end{array}$ & $\begin{array}{l}\text { Mean } \\
(\mathrm{SE})\end{array}$ & $\begin{array}{l}\text { Mean } \\
(\mathrm{SE})\end{array}$ & $\begin{array}{l}\text { Mean } \\
(\mathrm{SE})\end{array}$ & $\mathrm{F}$ & P-value \\
\hline \multirow[t]{2}{*}{ Red-eyed Vireo $^{\text {ce }}$} & 0.50 & 1.00 & 0.50 & 0.54 & 0.69 & 1.23 & 0.64 & 1.00 & 1.20 & 4.03 & 0.02 (1997) \\
\hline & $(0.22)$ & $(0.36)$ & $(0.24)$ & $(0.18)$ & $(0.17)$ & $(0.17)$ & $(0.09)$ & $(0.11)$ & (0.09) & 0.65 & 0.53 (1998) \\
\hline \multirow[t]{2}{*}{ Scarlet Tanager ${ }^{\mathrm{d}}$} & 0.17 & 0.67 & 1.00 & 0.31 & 0.38 & 0.23 & 0.20 & 0.27 & 0.26 & 5.03 & 0.01 \\
\hline & $(0.17)$ & $(0.21)$ & $(0.36)$ & $(0.13)$ & $(0.14)$ & $(0.12)$ & $(0.06)$ & $(0.06)$ & $(0.06)$ & & \\
\hline \multirow[t]{2}{*}{ Veery } & 0.67 & 0.33 & 0.00 & 0.46 & 0.61 & 0.31 & 0.41 & 0.44 & 0.40 & 0.65 & 0.53 \\
\hline & $(0.33)$ & $(0.21)$ & $(0.00)$ & $(0.14)$ & $(0.14)$ & $(0.17$ & $(0.09)$ & $(0.08)$ & (0.08) & & \\
\hline
\end{tabular}

${ }^{a}$ ANCOVA, testing for treatment effects with pre-harvest abundance as a covariate.

${ }^{\mathrm{b}}$ Significantly higher in unharvested than in peripheral and harvested areas (Scheffe' multiple comparison test, family alphalevel=0.10).

${ }^{c}$ ANCOVA, with vertical structure as a covariate.

${ }^{\mathrm{d}}$ Significantly higher abundance in harvested than peripheral or unharvested areas (Scheffe' multiple comparison test, family alphalevel=0.10).

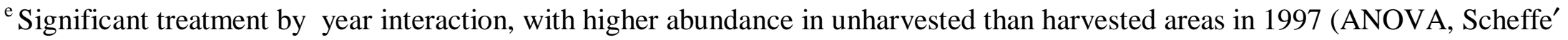
multiple comparison test, family alpha-level=0.10). 
Table 3.4. Means and standard errors (SE) for the abundance of nesting guilds and habitat groups at harvested, peripheral, and unharvested stands one year before harvest (1996) and two years after (1997-98) on the Westvaco Wildlife and Ecosystem Research Forest, Randolph County, West Virginia. Means are for untransformed data and are not adjusted for other terms in the model.

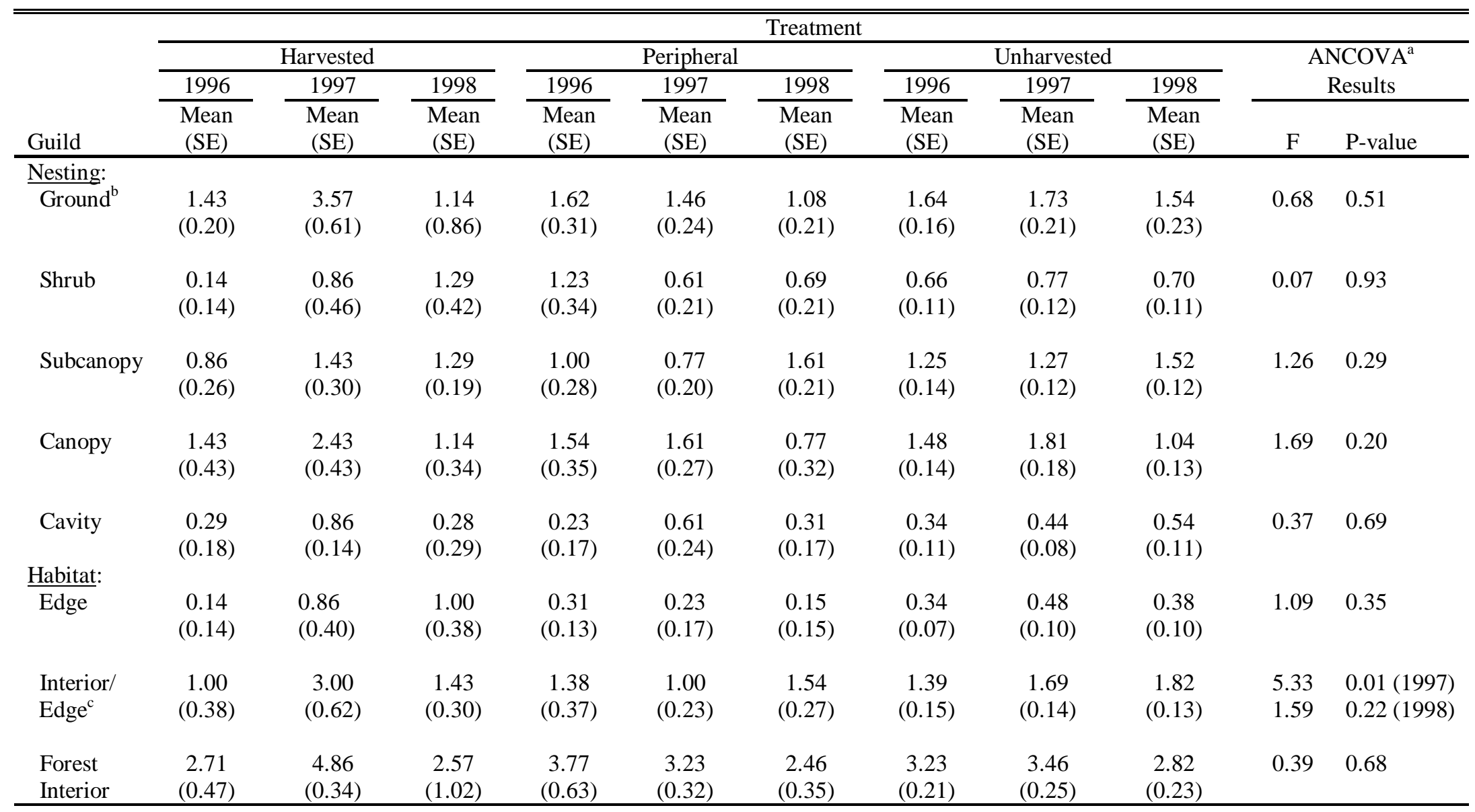

${ }^{a}$ ANCOVA, testing for treatment effects with pre-harvest abundance as a covariate.

${ }^{\mathrm{b}}$ ANCOVA, with vertical structure as a covariate.

${ }^{\text {c }}$ Significant treatment by year interaction, with higher abundance in harvested than unharvested or peripheral areas in 1997 (Scheffe' multiple comparison test, family alpha-level=0.10). 
Table 3.5. Means and standard errors for habitat measurements on two-age harvested stands and on unharvested stands on the Westvaco Wildlife and Ecosystem Research Forest, Randolph County, West Virginia, 1998. Means are for untransformed data.

\begin{tabular}{|c|c|c|c|c|c|c|}
\hline \multirow[b]{3}{*}{ Variable } & \multicolumn{4}{|c|}{ Treatment } & \multirow[b]{3}{*}{$\mathrm{F}$} & \multirow[b]{3}{*}{ P-value } \\
\hline & \multicolumn{2}{|c|}{ Harvested } & \multicolumn{2}{|c|}{ Unharvested } & & \\
\hline & Mean & $\mathrm{SE}$ & Mean & $\mathrm{SE}$ & & \\
\hline \multicolumn{7}{|c|}{ Woody spp. (no./ha): } \\
\hline Shrubs & 6281.2 & 1114.1 & 3411.5 & 562.7 & 3.92 & 0.07 \\
\hline Saplings & 1468.7 & 602.1 & 2207.3 & 353.7 & 0.42 & 0.53 \\
\hline Poles & 302.1 & 172.4 & 931.2 & 100.9 & 7.83 & 0.01 \\
\hline \multicolumn{7}{|l|}{ Trees (no./ha): } \\
\hline$>8-14.9-\mathrm{cm}$ & 42.7 & 20.0 & 156.5 & 11.7 & 14.79 & $<0.01$ \\
\hline$>15-22.9-\mathrm{cm}$ & 21.9 & 12.0 & 86.9 & 6.5 & 8.05 & 0.01 \\
\hline$>23-29.9-\mathrm{cm}$ & 43.7 & 21.3 & 89.2 & 7.9 & 0.76 & 0.40 \\
\hline$>30-37.9-\mathrm{cm}$ & 31.2 & 15.0 & 67.9 & 6.2 & 1.52 & 0.24 \\
\hline$>38-\mathrm{cm}+$ & 29.2 & 13.2 & 82.3 & 4.8 & 7.82 & 0.01 \\
\hline Snags & 21.9 & 13.8 & 46.0 & 7.2 & 0.58 & 0.46 \\
\hline
\end{tabular}

Cover variables (\%):

\begin{tabular}{lrrrrrr}
$>0.5-3-\mathrm{m}$ & 23.9 & 8.2 & 42.6 & 2.7 & 2.03 & 0.07 \\
$>$ 3-6-m & 18.7 & 8.0 & 42.5 & 2.5 & 6.99 & 0.02 \\
$>$ >6-12-m & 11.2 & 5.4 & 42.6 & 2.0 & 17.00 & $<0.01$ \\
$>$ 12-18-m & 12.3 & 3.8 & 43.1 & 1.9 & 20.08 & $<0.01$ \\
$>$ 18-24-m & 19.0 & 7.1 & 50.9 & 2.8 & 8.52 & 0.01 \\
$>$ 24-m & 17.3 & 8.6 & 48.0 & 3.5 & 5.47 & 0.03 \\
Green & 36.7 & 3.5 & 23.8 & 2.3 & 4.83 & 0.04 \\
Litter & 22.7 & 7.0 & 58.5 & 2.7 & 11.08 & $<0.01$ \\
Down Wood & 16.7 & 1.9 & 8.9 & 0.8 & 15.93 & $<0.01$ \\
Bareground/rock & 22.5 & 6.0 & 8.5 & 1.5 & 1.73 & 0.21 \\
\hline
\end{tabular}




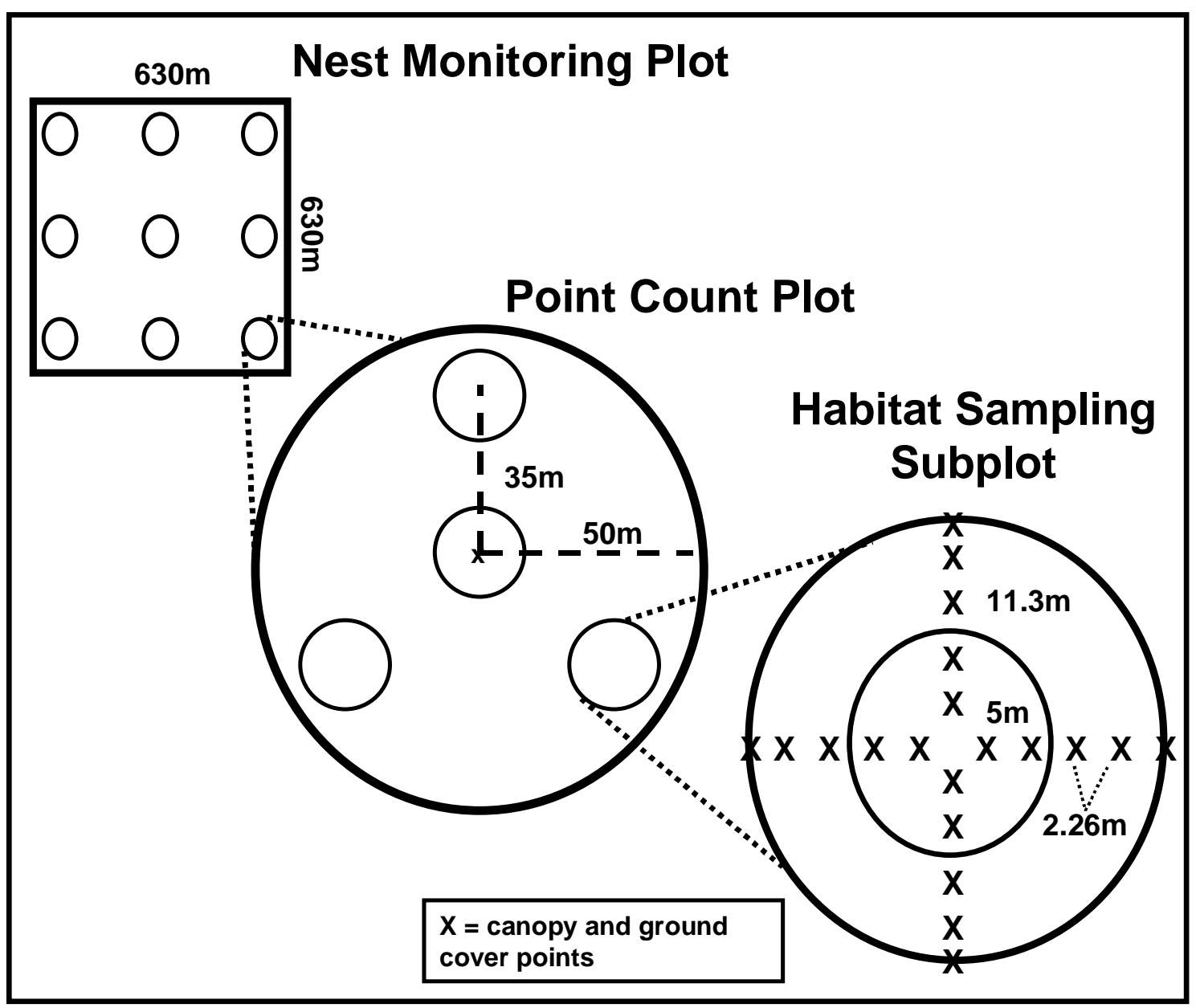

Figure 3.1. Layout-out of nest searching plot, point count plots, and habitat sampling subplots on the Westvaco Wildlife and Ecosystem Research Forest, Randolph County, West Virginia, 1996-1998. 


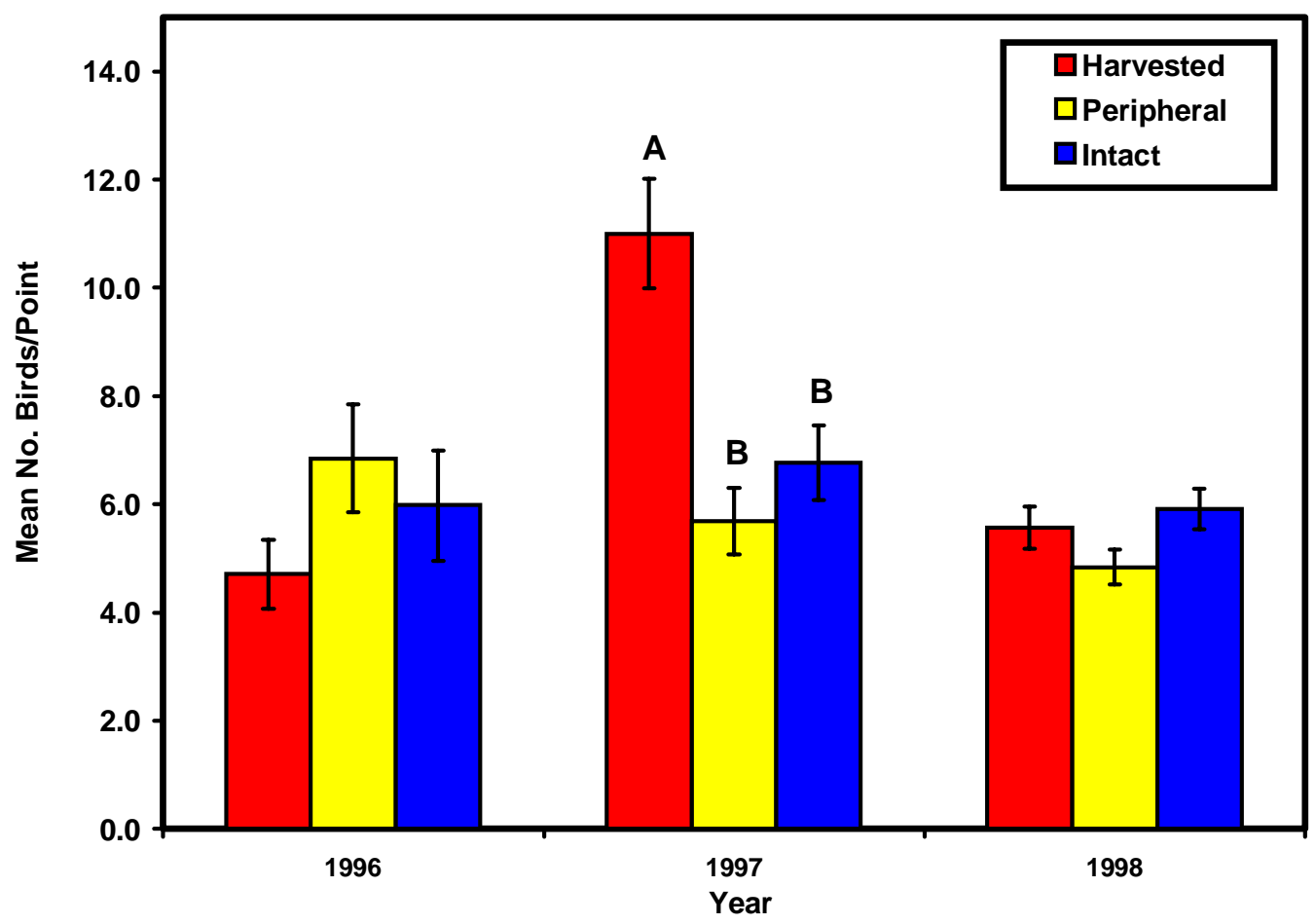

Figure 3.2. Mean total abundance of songbirds at harvested, peripheral, and intact (unharvested) stands on the Westvaco Wildife and Ecosystem Research Forest, Randolph County, West Virginia, 1997-98. Bars with different letters differ at $\mathbf{P} \leq \mathbf{0 . 1 0 .}$ 


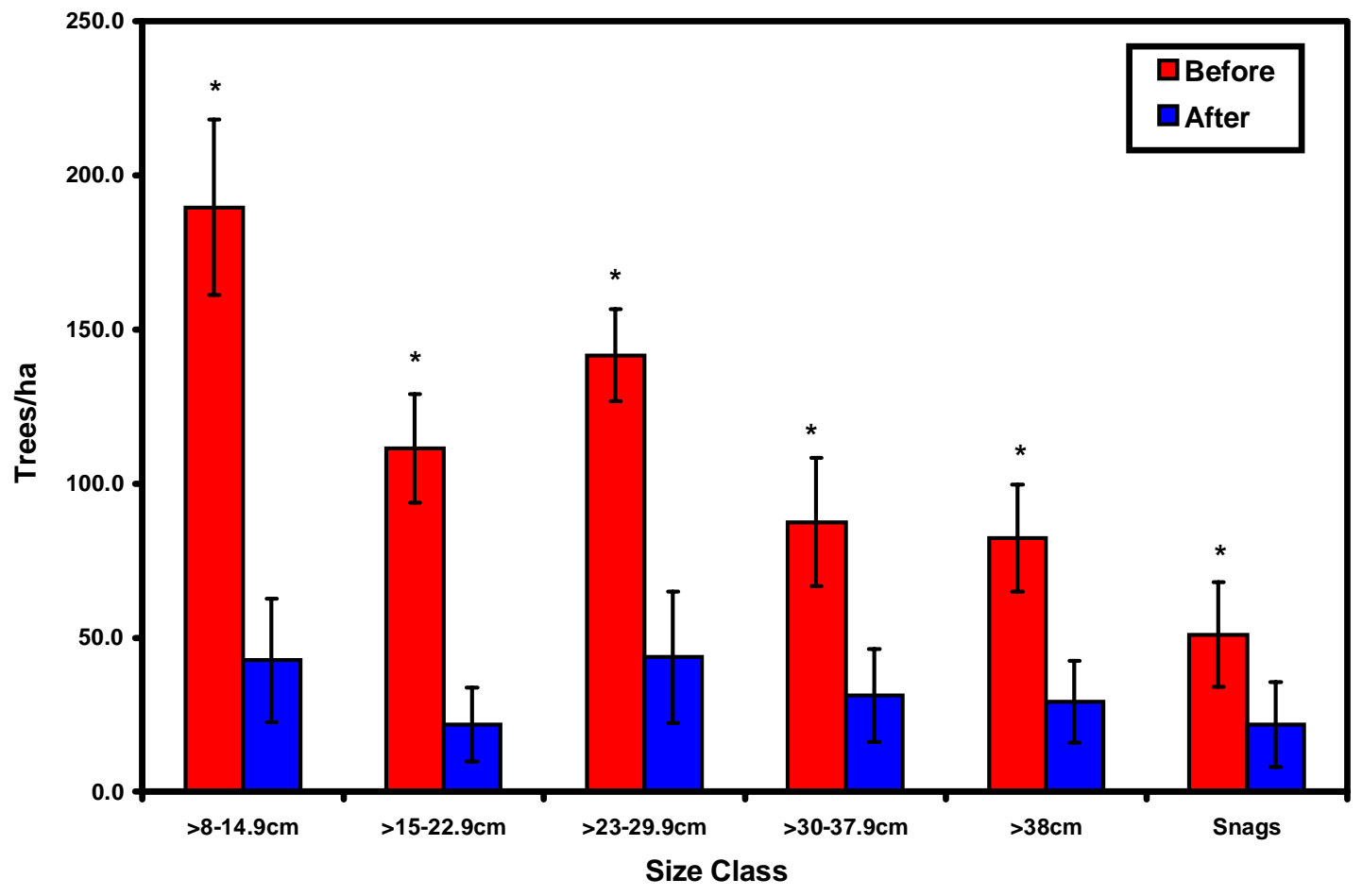

Figure 3.3. Mean density of trees in five size classes one growing season before (1996) and two growing seasons after harvesting (1998) on two-age stands on the Westvaco Wildife and Ecosystem Research Forest, Randolph County, West Virginia. Error bars represent \pm 1 standard error. Bars with an asterisk differ before and after harvesting at $P \leq 0.10$. Means are for untransformed data. 


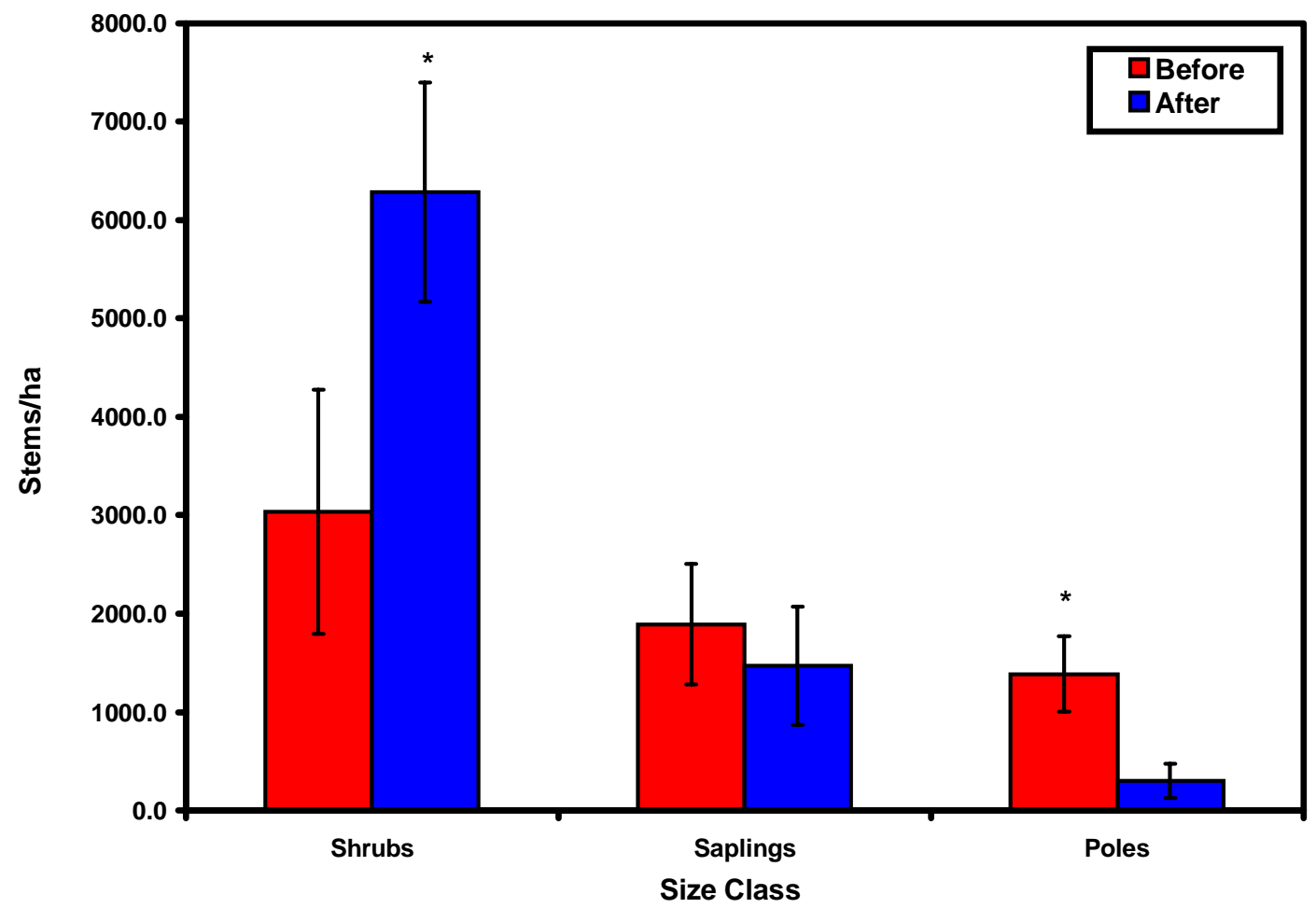

Figure 3.4. Mean density of shrubs, saplings, and one growing season before (1996) and two growing seasons after harvesting (1998) on two-age stands on the Westvaco Wildlife and Ecosystem Research Forest, Randolph County, West Virginia. Error bars represent \pm 1 standard error. Bars with an asterisk differ before and after harvesting at $\mathbf{P} \leq \mathbf{0 . 1 0}$. Means are for untransformed data. 


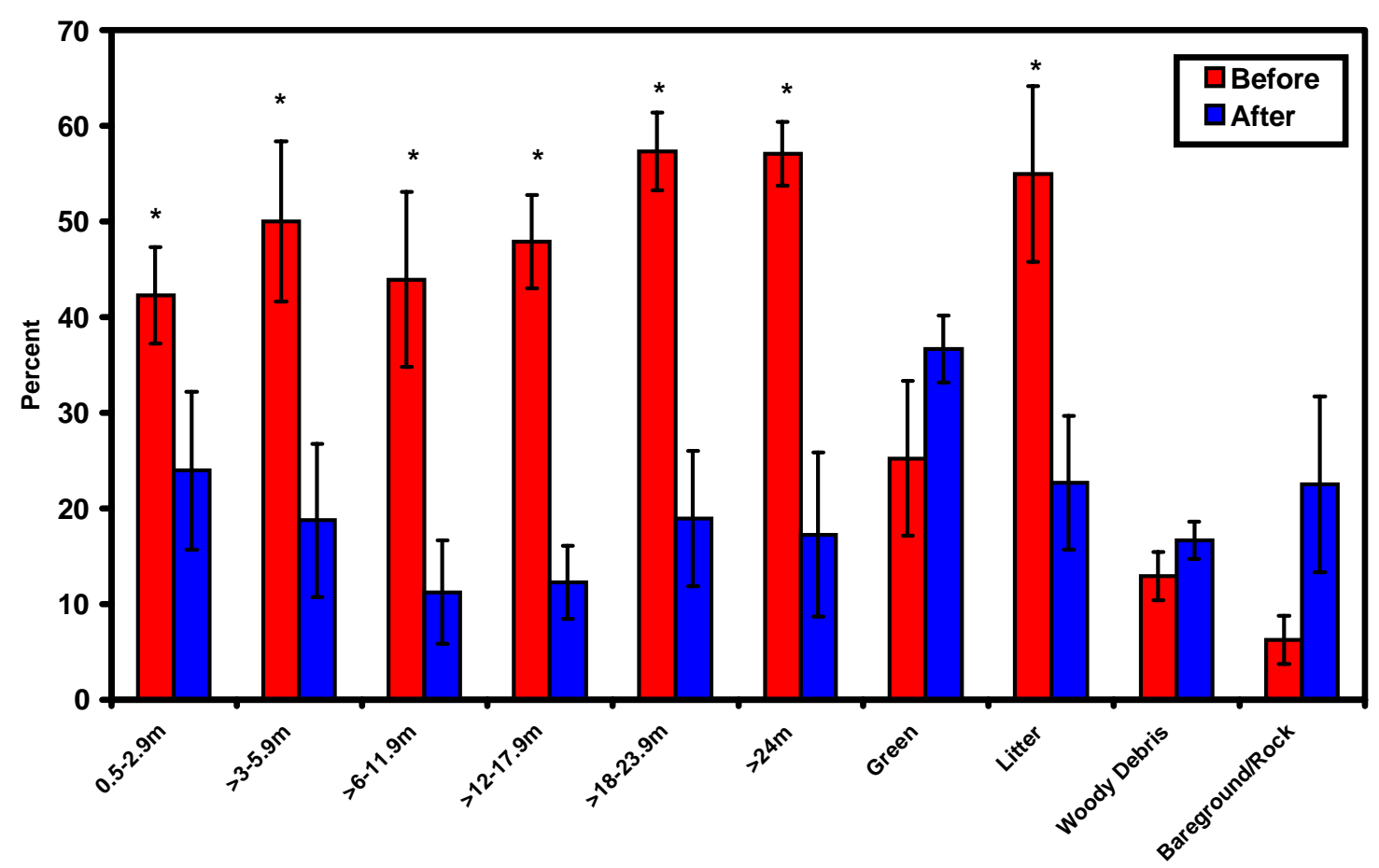

Cover Variable

Figure 3.5. Mean percent cover in six canopy layer classes and four ground cover classes one growing season before (1996) and two growing seasons after harvesting (1998) on two-age stands on the Westvaco Wildlife and Ecosystem Research Forest, Randolph County, West Virginia. Error bars represent \pm 1 standard error. Bars with an asterisk differ before and after at $\mathbf{P} \leq \mathbf{0 . 1 0 .}$ Means are for untransformed data. 


\section{CHAPTER 4}

LANDSCAPE AND MICROHABITAT FACTORS INFLUENCING SONGBIRD

OCCURRENCE ON AN INDUSTRIAL FOREST IN

CENTRAL WEST VIRGINIA 


\section{ABSTRACT}

Many studies examining forest fragmentation effects on songbirds have been conducted in areas where the landscape was significantly altered either by urbanization or agricultural practices rather than in landscapes where extensive forest is the dominant habitat. There is some evidence that forest fragementation caused by timber harvesting has different effects on bird abundance than fragmentation due to non-timber land uses. Also, it is unclear as to whether microhabitat-level or landscape-level characteristics are more important predictors of breeding bird occurrence in the central Appalachians. Thus, the objective of this study was to determine the specific landscape-level and microhabitat-level features that affect songbird abundance on a recently fragmented industrial forest in the Appalachians. I examined songbird populations at 50-m fixed-radius point counts on the Westvaco Wildlife and Ecosystem Research Forest in Randolph County, West Virginia, 1-yr before and 1-2-yr after harvesting. Microhabitat variables were measured within $6950-\mathrm{m}$ radius point count plots, and 1-km radius landscapes around each point count were analyzed using FRAGSTATS software. Contingency table analyses were used to examine the frequency of occurrence of songbirds in fragmented and nonfragmented landscapes. Stepwise logistic regression was used to model the effects of landscape composition and configuration and the effects of microhabitat variables on the probability of species occurrence both before and after the harvesting event. Results indicate that both landscape and microhabitat variables are important predictors of occurrence of most species and responses are species-specific. To ensure adequate habitat for both early and late successional species, forest managers should use both even and uneven-aged management to plan for songbird habitat.

Key Words: forest fragmentation, landscape ecology, microhabitat, songbirds, 


\section{INTRODUCTION}

Habitat fragmentation and ecosystems management from a landscape-level perspective have become major areas of focus in conservation biology (Harris 1984, Petit et al. 1995). It has been suggested that forest fragmentation has negative effects on the abundance, diversity, and reproductive success of forest-interior songbird populations (Finch 1991, Faaborg et el. 1995, Robinson et al. 1995). Fragmentation may negatively affect forest-dwelling songbirds because of isolation effects, area effects, edge effects, and competitive species interactions (Finch 1991, Faaborg et al. 1995).

In a forested landscape, fragmentation results from timber harvests, roads, powerlines, stand diversity, and natural canopy gaps. This is a much finer scale than occurs in agricultural areas, where forests appear as "islands" in a sea of crops and/or pastureland. Fragmentation on industrial forest might be viewed as "internal" or soft fragmentation, whereas fragmentation in an agricultural landscape might be viewed as "external" or hard fragmentation (Hunter 1990). Fragmentation in an agricultural landscape is often permanent, but fragmentation in forested landscapes is usually temporary (Faaborg et al. 1995). Faaborg et al. (1995) suggest that the latter type of fragmentation is less severe to forest birds than permanent fragmentation, but nonetheless, "detrimental effects still exist."

Previous research suggests that a high amount of edge habitat might be detrimental to songbird species (see Paton 1991 for a review). These studies suggest that songbirds are attracted to edges for nesting, but incur higher nest predation rates and brown-headed cowbird (Molothrus ater) parasitism than in other habitats. However, these edge effects likely only occur $<25-\mathrm{m}$ into forest Paton (1991). Moreover, it has been determined that higher rates of predation near edges occurred more frequently in fragmented landscapes than in forested landscapes (Hartley and Hunter 1998). Brown-headed cowbird parasitism also appears to be more detrimental to songbirds in fragmented landscapes than in contiguous forest (Donovan et al. 1995, Hagan et al. 1997).

Many studies have examined the effects of forest fragmentation on songbirds in areas where the landscape was significantly altered either by urbanization, suburbanization, or agricultural practices rather than in landscapes where extensive forest is the dominant habitat type (Faaborg et al. 1995, Hagan et al. 1996). Often these studies were conducted several years or decades after the fragmentation event had occurred. Accordingly, these studies were 
conducted in areas where fragmentation had become the dominant landscape configuration (Hagan et al. 1996). Few studies have attempted to determine how fragmentation events immediately impact songbirds

The immediate effects of fragmentation of a previously forested landscape in the temperate zone on songbirds has been investigated by Hagan et al. (1996). They compared songbird abundance in areas that had been fragmented within the last 3 years to areas that were nonfragmented. Densities for several species were higher in fragments, and pairing success and productivity of one species, the ovenbird (Seirus aurocapillus), were lower in newly created fragments. It was postulated that although densities may increase in newly formed fragments, breeding success may decrease because of increased competition for resources between individuals (Hagan et al. 1996). In a similar study, songbird populations were examined in areas of moderate canopy disturbance on an unfragmented forest in Vermont (Buford and Capen 1999). They found that most songbird species did not differ in abundance between sites with disturbed canopy and sites without disturbance.

Bird populations in forests in Missouri with and without clearcutting were examined by Thompson et al. (1992). Some forest-interior species were less abundant on the forests with clearcutting and some were more abundant, whereas early successional species all were more abundant in forests with clearcutting. Avian nest predator abundance and cowbird parasitism did not differ between the forest sites. In addition, forest-interior birds extensively used early and mid-successional even-aged stands. Fragmentation caused by timber harvesting may be less severe than fragmentation due to land conversion.

Previous research has focused on forests where the primary form of forest fragmentation was clearcutting (Thompson et al. 1992, Hagan et al. 1996). In other heavily forested regions of the East, other silvicultural systems, such as two-age harvesting, group selection, and single-tree selection, are being used. Less is known about these partial-harvesting techniques and how they impact birds returning to their breeding territories. Another common timber removal technique is diameter-limit, or sawtimber harvesting, where all trees greater than a pre-determined diameter at breast height (dbh) are removed. Although this method is not a scientifically-based silvicultural system, it is the most commonly used technique for removing trees from private lands in the eastern deciduous forest on private lands (Miller 1993). 
Songbirds also are known to respond to structural characteristics of the microhabitat, such as foliage height diversity and stem density (MacArthur and MacArthur 1961, James 1971, Anderson and Shugart 1974, Willson 1974, Smith 1977, Whitmore 1979, Bell and Whitmore 1997). Songbird habitat selection likely occurs at multiple scales (Dunning et al. 1992), and thus, both landscape- and microhabitat-level characteristics should be used when developing habitat models for birds.

At present, little is known about the immediate response of songbirds to timber harvesting as a fragmentation event within forested landscapes. Furthermore, it is unclear whether microhabitat-level or landscape-level characteristics are more important predictors of breeding bird occurrence in the central Appalachians. The objectives of this study were to compare songbird abundance in forest fragmented by harvesting to forest that is not fragmented and to determine the specific landscape-level and microhabitat-level features of a recently fragmented industrial forest landscape that affect songbird abundance. Accordingly, I examined songbird abundance, and landscape- and microhabitat-level characteristics on an industrial forest in the central Allegheny mountains of West Virginia. Data were collected over a 3-year period as the forest became increasingly fragmented by timber harvesting practices.

\section{STUDY AREA}

The Westvaco Wildlife and Ecosystem Research Forest (WWERF) in Randolph County, West Virginia ( $38^{\circ} 42^{\prime} \mathrm{N}$ lat., $80^{\circ} 3^{\prime} \mathrm{W}$ long.) was the study area for this research . This 3360-ha working forest was designated by Westvaco in 1994 as a center for the study of the effects of timber harvesting on Appalachian ecosystems. The forest is located in the Allegheny Mountain Section of the Central Appalachian Broadleaf-Coniferous Forest Province (Fenneman 1938), and elevations range from 740-m to 1200-m. The climate is generally cool and moist (Strausbaugh and Core 1977), and the northern hardwoods forest type was the predominant cover type at the start of the study. Tree species included red maple (Acer rubrum), sugar maple (A. saccharum), black cherry (Prunus serotina), American beech (Fagus grandifolia), and yellow birch (Betula allaheniensis). A small percentage of the forest at low elevations was in the cove hardwood forest type dominated by tuliptree, American basswood (Tilia americana), and northern red oak (Quercus rubra). In higher exposed elevations and sheltered riparian areas, red spruce (Picea rubra), and eastern hemlock (Tsuga canadensis) predominated. Some upland hardwoods, 
dominated by chestnut oak $(Q$. prinus $)$ and scarlet oak $(Q$. coccinea $)$ were found on dry slopes and ridges. Common understory vegetation included striped maple (A. pensylvanicum) and witchhazel (Hamamelis virginia). Thickets of rhododendron (Rhododendron maxiumum) were found along streams and rock outcrops. Some previously logged stands had dense ground covers of hay-scented fern (Dennstaedtia punctilobula) or hedged greenbrier (Smilax spp.) because high white-tailed deer (Odocoileus virginianus) herbivory on the forest. Additionally, a small percentage of the forest is open/nonforested areas composed of open-canopy roads and surface mines (Fig. 4.1).

Several timber harvests were conducted on the WWERF prior to the start of this study in May 1996. Diameter-limit harvesting occurred on 2 stands in 1994 and on 1 stand in early 1996. Two-age harvesting occurred on 2 stands, 1 in 1990 and 1 in 1994 . One stand received a seed tree harvest in 1991, and another stand received a seed tree harvest immediately before the first field season (Fig. 4.1). Diameter-limit stands had an average basal area of $20 \mathrm{~m}^{2} /$ ha after harvest, and two-age stands had an average basal area of $9.5 \mathrm{~m}^{2} /$ ha after harvest. Diameter-limit harvests had scattered openings from the removal of overstory trees $>45.7-\mathrm{cm} \mathrm{dbh}$. Residual trees in two-age stands were scattered relatively evenly throughout the stand, but some trees were clumped along spring seeps and intermittent drainages.

Between the 1996 and 1998 field seasons several stands were harvested using a diameterlimit, 2 stands were harvested using a two-age technique, and 2 stands were harvested using a seed tree (regeneration) technique (Fig. 4.1). In addition, several small blocks were harvested in 3 stands using a variety of harvesting methods (clearcut, two-age, shelterwood harvests) for a study examining impacts of forest management techniques on floral diversity and woodland salamanders (Harpole and Haas 1999).

\section{METHODS}

\section{Songbird Abundance}

Songbird abundance was measured on the WWERF using standardized point count procedures (Ralph et al. 1993). I used 50-m fixed radius point counts to sample the songbird community. Each point count was surveyed twice between 20 May and 5 July each year of the study, once each by 2 different observers. The maximum number of birds detected between these 2 counts was recorded as the index of abundance for each point count (Hagan et al. 1997, 
Nur et al. 1999). For example, if 1 Canada warbler (Wilsonia canadensis) was detected in the first session, and 3 were detected in the second, 3 was used as the index of abundance.

Counts began at one-half hour after sunrise (approximately 0600 EST) and lasted until 4 hours after sunrise (approximately 1000 EST). All birds seen or heard singing/calling were recorded as either in or out of the 50-m radius circle. Counts lasted 10-min and began approximately 1-min after the observer reached the point count station. Counts were not conducted during rain or when noise from wind interfered with counting.

Point counts were located on a 241-m x 241-m grid system that had been established previously for forest inventory purposes by Westvaco personnel in 1995. Points were marked with an aluminum stake and uniquely numbered. A Trimble Global Positioning System ${ }^{\mathrm{TM}}$ (GPS) was used to geographically reference points. Sixty-nine points were placed 241-m apart (every grid point) within eight 39.7-ha nest searching plots. An additional 74 points were located throughout the forest and were separated by a distance of at least 482-m (every other grid point on every other line) in order to cover the entire area. The first line sampled was selected randomly as were the first point on each line. I attempted to sample every other grid point on the line thereafter. Point count stations were found to be spatially independent (Weakland and Wood, unpub. rept.).

Observers were trained for a period of 2-3 days before starting actual surveys. Two observers conducted counts at the same station and compared species observed and distances estimated. They also practiced distance estimation by pacing 50-m from the point count center after the practice session and by pacing to the approximate location of a singing male bird. At least ten practice sessions were conducted before beginning actual surveys.

\section{Landscape analysis}

Landscape analysis was conducted for each year of the study using a GIS (geographic information system) of the WWERF developed by Westvaco using USGS topographic maps and Westvaco FRIS ${ }^{\text {TM }}$ (Forest Resources Information System) data. Arcview 3.0 was used to examine shape files of forest stands and to alter attributes to reflect the following 5 cover classes: mature northern hardwoods, mature mixed woods (northern hardwoods and conifers), regeneration harvests (seed tree and two-age), diameter-limit harvests, and open-nonforested areas. Mature hardwoods were a combination of northern hardwoods and upland hardwoods because too few points were in upland hardwoods to analyze separately. Mature mixed woods 
were a mix of hardwoods, eastern hemlock, and/or red spruce. These stands originally were classified by Westvaco as either cove forests, upland hardwoods, or northern hardwoods, but I chose to separate them into a mixed woods category because of the presence of conifer cover in the stand based on aerial photographs and ground truthing. Seed tree and two-age regeneration harvests were combined because they had similar structure, canopy cover, and basal area. Open/nonforested areas included roads, log landings, and surface mines. Shape files for each year were converted to ArcInfo coverages and rasterized. One kilometer radius circles were electronically "clipped" around each point count (Hagan et al. 1997). The clipped images were used in subsequent analyses of landscape composition and configuration (Hagan et al. 1997).

Landscape metrics were computed for each year using the raster version of FRAGSTATS Version 2.0 software (McGarigal and Marks 1992) to quantify the increasing amount of fragmentation of the forest as a result of timber harvesting. Composition metrics included percent cover of the 5 cover classes and percent core area of mature forest. Percent core area (\%Core) of each cover class and contrast-weighted edge density (CWED) were calculated using a 100-m buffer (McGarigal and Marks 1992). Percent core area of mature forest was the sum of the percent core area of mature hardwoods and mature mixedwoods.

Landscape configuration variables included CWED and number of mature forest patches (NP). The amount of edge contrast between habitat types may have varying degrees of effect on songbird populations (McGarigal and Marks 1992). Each combination of cover classes was given a weight for use in calculations of edge density (Table 4.1). Weights ranged from 0 to 1 , with 1 indicating the highest amount of contrast between classes. This index quantifies edge from its functional significance to songbird species and incorporates both edge density and edge contrast (McGarigal and Marks 1992). The index reduces the length of each edge segment in proportion to its degree of contrast. Edge density for a maximum-contrast edge (weight=1.0) would not be affected, but edge density for edges with lower weights will be reduced. For example, a 100-m/ha edge with a weight of 1.0 will not be affected, but an edge with a weight of 0.25 would be reduced by $75 \%$ to $25-\mathrm{m} /$ ha (McGarigal and Marks 1992). Number of patches is simply the number of distinct patches of each class within the landscape (McGarigal and Marks 1992). I included the number of patches of mature forest as an index of habitat configuration, because I was primarily concerned with forest-dwelling songbirds. A higher value for either contrast-weighted edge density or number of patches indicates a higher amount of fragmentation. 


\section{Microhabitat Sampling}

Habitat characteristics were measured within each point count circle on nest searching plots in 1996. Point count circles that were harvested were re-sampled in 1998. Four 0.04-ha subplots were established within the 50-m radius point count circle. One subplot was centered on the grid stake, and the other 3 were located $35-\mathrm{m}$ away at $0^{\circ}, 120^{\circ}$, and $240^{\circ}$ (Fig. 4.2). Subplots had a radius of $11.3-\mathrm{m}$ in which all trees $>8-\mathrm{cm}$ were counted and placed into $2 \mathrm{dbh}$ size categories: $>8-29.9-\mathrm{cm}$ and $\geq 30-\mathrm{cm} \mathrm{dbh}$. The number of snags $>12.0-\mathrm{cm}$ also were counted within the $11.3-\mathrm{m}$ radius circle. The number of understory stems $\leq 8-\mathrm{cm}$ dbh and $>0.5$ $\mathrm{m}$ high were counted within a 5.0-m radius circle of the subplot center (Fig. 4.2).

The James and Shugart (1970) ocular site-tube method was used to estimate the percent canopy cover in 6 layer classes: >0.5-3-m, >3-6-m, >6-12-m, >12-18-m, >18-24-m, and >24-m. A vertical structure index (VSI) was calculated for each point count circle using the percent cover in each of these layer classes (Nichols 1996). Ground cover was estimated in a similar manner as canopy cover, with cover categories of: green vegetation (which included grasses, ferns, moss, herbs, and seedlings), leaf litter, woody debris, and bareground/rock. Woody debris was any downed $\log$ or branch $\geq 4.0$-cm in diameter; smaller woody debris was counted as litter. Twenty presence/absence site-tube readings were taken every $2.26-\mathrm{m}$ along two $22.6-\mathrm{m}$ perpendicular transects bisecting the center of the plot, once for each canopy layer and once for ground cover (Fig. 4.2). Transects were established so that 1 was oriented perpendicular to the slope and that 1 was parallel to the slope (Martin et al. 1997).

\section{Data Analyses}

\section{Contingency Table Analysis}

To compare songbird abundance in fragmented and nonfragmented landscapes I used contingency table analysis (Zar 1999). I determined whether there is an association between species presence/absence and landscape condition while controlling for years (Stokes et al. 1995). I used a categorical presence/absence variable rather than absolute abundance because most point counts did not have more than 1 individual of a species within the 50-m radius plot. Hagan et al. (1996) used a similar approach in their study of fragmentation effects on songbirds in Maine. I conducted 2 analyses to determine if different harvest types act as fragmentation events. In the first analysis ( $2 \times 2$ contingency table), I used the sum of the amounts of nonforested habitat, diameter-limit harvests, and regeneration harvests to classify landscapes as 
fragmented or nonfragmented. A landscape was considered fragmented if the sum of these three classes was $>10 \%$ of the landscape.

In the second analysis ( 2 x 3 contingency table) I examined the effect of different harvest types as fragmentation events. The categorical variables for landscape condition were: fragmented by diameter-limit, fragmented by regeneration, and nonfragmented. Nonfragmented landscapes had $<10 \%$ diameter-limit harvest and $<10 \%$ regeneration harvest cover. Landscapes fragmented by diameter-limit harvests had $>10 \%$ diameter-limit harvest cover and $<10 \%$ regeneration harvest cover. Landscapes fragmented by regeneration harvests had $>10 \%$ regeneration harvest and $<10 \%$ diameter-limit harvest cover.

For both analyse, I controlled for year and examined the Cochran-Mantel-Haenszel chisquare statistic for significance, and only bird species that were detected in at least 5 landscapes in each treatment were included in these analyses (Stokes et al. 1995). Results were considered significant at $\mathrm{P} \leq 0.10$. The conservative $\mathrm{P}$-value of 0.10 was chosen in order to minimize the probability of a Type II error, which may be more important in ecological situations than minimizing a Type I error (Askins et al., 1990; Johnson, 1999). Error rates were controlled for all treatment comparisons for a bird species, but not experiment-wide for all species. If a significant result was found in the second analysis, I performed all 3 subsequent pairwise tests to determine specific differences between landscape conditions. Significance for these tests was $\alpha / 3$ or $\mathrm{P} \leq 0.03$. The $\mathrm{SAS}^{\circledR}$ system for statistical analysis (Proc Freq) was used to conduct all contingency table analyses (SAS ${ }^{\circledR}$ Institute 1991).

\section{Logistic Regression}

I used stepwise logistic regression (Neter et al. 1996) to examine the relationship between landscape and microhabitat level characteristics and the presence of songbird species. The significance level chosen for entry and retention in the model was 0.10 . I used presence/absence as the dependent variable because at most point counts only 1 individual of a species was detected within 50-m (Hagan et al. 1997). This technique was chosen because it has been used by other researchers examining the effects of landscapes on songbird species (Hagan et al. 1997, Villard et al. 1999), and because predictor variables do not need to follow a joint multivariate normal distribution (Neter et al. 1996). Models were fit for a species if it was detected at $\geq 20 \%$ or $\leq 80 \%$ of point counts each year to ensure an adequate sample size (R. Harner, pers. comm.). 
I modeled the effects of landscape and microhabitat characteristics both separately and combined. Landscape models were created using 8 variables measured on all point counts each year that had GPS coordinates $(n=143)$ : contrasted-weighted edge density (CWED), number of mature forest patches (NP), percent core area of mature forest (\%Core), percent mature hardwood (\%Hard), percent mature mixedwood (\% Mix), percent diameter-limit harvest (\%Diam), percent regeneration (\%Regen), and percent open/nonforested (\%Open).

Microhabitat models were created using 8 variables measured on 69 point counts: density of trees $>8-29.9-\mathrm{cm} \mathrm{dbh}$ (Stree), density of trees $\geq 30-\mathrm{cm}$ dbh (Ltree), density of understory stems $\leq 8$-cm dbh (Under), VSI, percent cover of green vegetation (Green), percent cover of leaf litter (Litt), percent cover of woody debris (Wood), and percent cover of bareground/rock (Bare). Combined models incorporating both microhabitat and landscape variables were created for all species using the 69 point counts where microhabitat characteristics were measured. Year was included in all models as a qualitative variable to account for annual variation in songbird abundance. Percentage data were subjected to arcsine-square root transformations. Tree, snag, and understory stem densities, and number of mature forest patches, were transformed using the $\log _{10}($ variable+1) transformation (Dowdy and Wearden 1991).

The likelihood ratio chi-square test was used to determine the significance of the addition of each variable to the logistic regression model (Stokes et al. 1995). Variables that failed the likelihood ratio chi-square test $(\mathrm{P} \leq 0.10)$ were not included in the model. The Wald chi-square test statistic was used to determine the importance of each variable in the model (Stokes et al. 1995). I compared the relative strength of landscape, microhabitat, and combined models by examining Hosmer-Lemeshow goodness-of-fit tests (Stokes et al. 1995). This is a conservative test that examines how well predicted values correspond to the observed values. A small test statistic and large P-value indicate a good fit. Models that failed the Hosmer-Lemeshow goodness-of-fit test $(\mathrm{P}<0.10)$ were rejected. The $\mathrm{SAS}^{\circledR}$ system for statistical analysis (Proc

Logistic) was used to conduct all logistic regression analyses (SAS ${ }^{\circledR}$ Institute 1991).

\section{RESULTS}

Four interior-edge songbird species and 11 forest-interior species were detected at $\geq 20 \%$ or $\leq 80 \%$ of point counts each year. Interior-edge species included: black-capped chickadee (Poecile atricapillus), dark-eyed junco (Junco hyemalis), eastern towhee (Pipilo 
erythrophthalmus), and rose-breasted grosbeak (Pheucticus ludovicianus); forest-interior species were: black-throated blue warbler (Dendroica caerulescens), Canada warbler (Wilsonia canadensis), magnolia warbler (D. magnolia), ovenbird (Seirus aurocapillus), scarlet tanager (Piranga olivacea), veery (Catharus fuscescens), and winter wren (Troglodytes troglodytes). Species nomeclature follows the American Ornithologists' Union checklist for North American birds (American Ornithologists' Union 1999).

\section{Contingency Table Analysis}

When all disturbances were considered, only black-throated blue warbler abundance differed among fragmented and nonfragmented landscapes (Table 4.2). This species was present at a higher proportion of points in nonfragmented landscapes than in fragmented landscapes.

All species, except the black-capped chickadee, differed among landscape types when diameter-limit and regeneration harvests were considered separately (Table 4.3). The dark-eyed junco was found in lower proportions in landscapes fragmented by regeneration harvests than in nonfragmented landscapes and landscapes fragmented by diameter-limit harvests. Canada warblers were found less frequently in nonfragmented landscapes than landscapes fragmented by diameter-limit harvests, whereas the winter wren was found more frequently in landscapes fragmented by diameter-limit harvests than in nonfragmented landscapes and landscapes fragmented by regeneration harvests. The black-throated blue warbler was found more often in landscapes fragmented by regeneration harvests than in either of the other 2 landscape types, and the ovenbird was found less frequently in landscapes fragmented by diameter-limit harvests. The scarlet tanager was found more frequently in nonfragmented landscapes than either of the other 2 landscape types; and the veery was found more often in nonfragmented landscapes than landscapes fragmented by regeneration harvests. The eastern towhee, rose-breasted grosbeak, and magnolia warbler were found in $<5$ landscapes fragmented by regeneration harvests and were not included in analyses.

\section{Logistic Regression}

\section{Landscape Models}

Landscape variables that differed between years were percent regeneration harvest, percent diameter-limit harvest, percent hardwood forest, and percent core of mature forest (Table 4.4). Percent regeneration was higher in 1997 and 1998 than in 1996 while percent core area 
was higher in 1996 than in 1997 or 1998. Percent diameter-limit harvest increased significantly each year, whereas percent hardwood forest declined each year.

Logistic regression models indicate that landscape configuration variables (CWED, NP, $\%$ Core) were significant predictors for presence of 3 interior-edge species: black-capped chickadees, dark-eyed juncos, and eastern towhees (Table 4.5). Abundances of Black-capped chickadees and dark-eyed juncos had negative relationships with CWED. Both dark-eyed junco and eastern towhee abundances were negatively related to \%Core. Landscape composition also influenced these two species. Dark-eyed juncos had positive relationships with \%Diam and $\%$ Hard, and eastern towhees had a positive relationship with \% Regen.

Among forest-interior species, only the ovenbird was positively related to \%Core (Table 4.5). Timber harvesting had an effect on several species. Percent regeneration was positively related to black-throated blue warbler, ovenbird, and scarlet tanager abundances, and winter wren abundance was negatively related to \%Regen. Other cover variables also influenced several species. The amount of open (nonforested) habitat was negatively related to blackthroated blue warbler abundance, and positively related to ovenbird abundance. Winter wren abundance also was positively related to \%Diam. Abundances of 2 species, the Canada warbler and magnolia warbler, were not related to any landscape variables.

\section{Microhabitat Models}

Descriptive statistics for microhabitat-level habitat characteristics are found in Table 4.6. Only 6 species had logistic regression models based solely on microhabitat variables (Table 4.7). Eastern towhee abundance was positively related to litter cover, and dark-eyed junco abundance was negatively related to understory stem density and positively related to percent green cover. Black-throated blue warbler abundance had negative relationships with density of trees >8-29.9$\mathrm{cm} \mathrm{dbh}$ and percent bareground, and veery abundance had a positive relationship with cover of woody debris. Abundance of Canada warblers was negatively related to litter cover, and abundance of magnolia warblers was negatively related to understory stem densities (Table 4.7).

\section{Combined Models}

Combined models indicated that most species respond to both landscape- and microhabitat-level characteristics (Table 4.8). Abundances of scarlet tanagers and winter wrens were related solely to landscape variables, while only veery abundance was related solely to 
microhabitat-level characteristics. The eastern towhee $\left(\mathrm{X}^{2}=10.17, \mathrm{P}<0.01\right)$ had a negative relationship to year, indicating that they declined from 1996 to 1998.

The combined models performed better than the landscape and microhabitat models for 1 forest-interior species (Table 4.9). Landscape models performed better than microhabitat and combined models for 2 interior-edge species and 3 forest-interior species, and microhabitat models performed better than landscape and combined models for 1 interior-edge species and 3 forest-interior species. An examination of Wald chi-square statistics indicates that for most species both microhabitat variables and landscape variables were important predictors of species presence or absence (Table 4.10).

\section{DISCUSSION}

Black-throated blue warblers and ovenbirds had higher abundance in forests fragmented by timber harvesting than in unfragmented forests in Maine (Hagan et al. 1996). I found that both these species had positive relationships with regeneration amount in the landscape. However, contingency analysis indicated that the ovenbird was not more frequently observed in landscapes fragmented by regeneration harvests than nonfragmented landscapes but was observed more frequently in nonfragmented landscapes than landscapes fragmented by diameterlimit harvests. Unlike Hagan et al. (1996) I found the black-throated blue warbler more frequently in nonfragmented landscapes than fragmented landscapes when all forms of disturbance were considered. However, this species found more frequently in forests fragmented by regeneration harvests than in forests fragmented by diameter-limit harvests or in nonfragmented forests. Thus, this species may be avoiding open areas such as roads and surface

mines. Analyses of the effects of partial harvesting methods on songbird abundance indicate that many species do not differ between harvested and unharvested stands on the WWERF (Chapters 1-3, this volume). My results might differ from Hagan et al. (1996) because the harvesting methods used on the WWERF left some canopy cover in the stand, while clearcuts in their study may have had all of the overstory removed. Another factor explaining differences between our results is that Maine is in a different biome (northern coniferous/transition forest) than West Virginia.

It appears that species respond to fragmentation by harvesting differently depending on the harvest method. Two species were found more frequently in nonfragmented landscapes than 
landscapes fragmented by regeneration harvests, whereas 2 different species were found more frequently in nonfragmented landscapes than landscapes fragmented by diameter-limit harvests. Hagan et al. (1996) suggest that forest-dwelling songbirds increase in fragmented areas as a result of displaced individuals packing into remaining habitat. However, displaced individuals also might avoid openings created by timber harvesting, and instead of settling in the closest available habitat they may choose to settle further away from the disturbance in nonfragmented forest. Some species might have a "psychological need" for larger forest (Hunter 1990). Thus, abundance of some species could increase in nonfragmented forest. Alternatively, songbird abundance may not differ between fragmented and nonfragmented areas if songbirds do not respond to timber harvesting as fragmentation and settle in the harvested area. I frequently found forest-dwelling songbirds in both regeneration and diameter-limit harvests (Chapters1-3, this volume). In Missouri, some forest-interior species had lower abundance on forest with clearcutting while others had higher abundance (Thompson et al. 1992). Forest-interior birds also extensively used early and mid-successional even-aged stands in forests in Missouri. Thus, songbird response to fragmentation by harvesting might vary by region and by harvest type.

Songbird presence was related to factors at both the landscape and microhabitat scales on the WWERF. Both landscape composition and configuration appeared to be important for several species. Several species had significant relationships to the amount of harvested habitat in the landscape. In southeastern Canada, ovenbirds and scarlet tanagers responded positively to forest cover and number of forest patches, whereas veeries responded negatively to total edge (Villard et al. 1999). In this study I observed similar trends for ovenbirds, but I could not fit a landscape model for veeries. However, the study by Villard et al. (1999) was in an area that has been permanently fragmented, and they were not examining amounts of different forest habitats, but rather the total amount of cover of forest.

In my study, most species had both landscape and microhabitat variables in their combined models, indicating that both levels should be considered when creating management plans for songbird species on the WWERF. However, studies by Buford and Capen (1999), working in unfragmented forest in Vermont, and by Demeo (1999), working on the relatively unfragmented Monongahela National Forest in West Virginia, indicate that landscape-level disturbances are less important predictors for bird species occurrence than local disturbance. However, landscape variables were more important predictors of occurrence than either 
macrohabitat or microhabitat features in fragmented cottonwood stands in Idaho (Saab 1999). Thus, songbird response to disturbance appears to vary across biomes and landscapes. In unfragmented eastern deciduous forests, microhabitat variables are likely more important predictors of occurrence than landscape variables. In forested landscapes fragmented by agriculture, landscape variables are likely more important, but regional differences are likely to occurr. The WWERF, as a managed, industrial forest, may represent an intermediate between these 2 extremes. Only recently has the WWERF been fragmented by timber harvesting, and large blocks of unfragmented forest still exist. Thus, both landscape and microhabitat variables were important predictors of species occurrence in my study.

\section{MANAGEMENT IMPLICATIONS}

Timber harvesting in the landscape does not appear to have immediate negative impacts on most common species of songbirds on the WWERF. Forest-interior songbird populations, however, may decrease if the amount of regeneration harvests, that are not regenerating because of deer herbivory, increases on the forest. Management for songbird diversity on the WWERF could be approached several ways. Forest managers could plan single-tree selection harvests to maintain canopy closure and increase vertical diversity for forest-interior species and species that use canopy gaps (Thompson et al. 1995). Selection harvesting will increase microhabitat variables, such as woody debris, herbaceous ground cover, and understory stem densities, that are important for several species. Species that were correlated to the amount of diameter-limit harvesting in this study would likely respond positively to the amount of single-tree harvests in the landscape. In addition to selection harvests, foresters also could harvest a few stands using seed tree and two-age harvests to increase habitat for early successional species, such as the eastern towhee, which was positively correlated to the amount of regeneration in the landscape (Thompson et al . 1995). Additionally, regeneration harvests could be "clumped" to decrease isolation effects and potentially to reduce deer herbivory (Thompson et al. 1995). Interior-edge species also would benefit from both selection harvests and regeneration harvests.

Another approach would be to increase the amount of regeneration harvests on the forest and increase rotation length to $>120$ years. In this approach, edge and early successional species would benefit from the increase in the amount of young and regenerating forest, which is a habitat that is scarce in West Virginia (Bell and Whitmore 1997). Forest interior species would 
benefit from the longer rotation lengths. Species such as the ovenbird that prefer high core area of mature forest would benefit from this approach. However, as stands mature some microhabitat variables, such as understory stem density, could decrease and lead to declines on some species. In both approaches, age classes should be balanced across the forest because each stage of succession is important for some species (Thompson et al. 1995).

\section{ACKNOWLEDGEMENTS}

This research was funded by Westvaco Corporation, Forest Resources Division, and the West Virginia Division of Natural Resources Nongame Wildlife and Natural Heritage Program. The West Virginia Cooperative Fish and Wildlife Research Unit, USGS, BRD, provided equipment and logistical support. Mark Ford, Sydney McIntyre, Sarah Clapham, and Sally Lane provided technical and field assistance. I would particularly like to thank Ann Steketee for her advice and help conducting landscape analyses. I also thank the many field technicians who assisted with nest searching, point count surveys, and habitat sampling. R. C. Whitmore, L. S. Gribko, A. F. Egan, and W. M. Ford provided helpful suggestions on earlier drafts of this manuscript.

\section{LITERATURE CITED}

AMERICAN ORNITHOLOGISTS' UNION. 1999. The American Ornithologists' Union check-list of North American birds. http://pica.wru.umt.edu/AOU/birdlist.HTML.

ANDERSON, S.H. AND H. H. SHUGART. 1974. Habitat selection of breeding birds in an east Tennessee deciduous forest. Ecology 55:828-837.

ASKINS, R.A., J.F. LYNCH, AND R. GREENBURG. 1990. Population declines in migratory birds in eastern North America. Curr. Ornith. 7: 1-57.

BELL, J.L. AND R.C. WHITMORE. 1997. Eastern towhee numbers increase following defoliation by gypsy moths. Auk 114: 708-716.

BUFORD, E. W., AND D. E. CAPEN. 1999. Abundance and productivity of forest songbirds in a managed, unfragmented landscape in Vermont. Journal of Wildlife Management 63:180-188. 
DEMEO, T. E. 1999. Forest songbird abundance and viability at multiple scales on the Monongahela National Forest, West Virginia. Ph.D. dissertation, West Virginia University, Morgantown, West Virginia. 159pp.

DONOVAN, T. M., F. R. THOMPSON III, J. FAABORG, AND J. R. PROBST. 1995. Reproductive success of migratory birds in habitat sources and sinks. Conservation Biology 9:1380-1395.

DOWDY, S. AND S. WEARDEN. 1991. Statistics for research. John Wiley and Sons. New York, New York. 629pp.

DUNNING, J. B., B. J. DANIELSON, AND H. R. PULLIAM. 1992. Ecological processes that affect populations in complex landscapes. Oikos 65:169-175.

FAABORG, J., M. BRITTINGHAM, T. DONOVAN, AND J. BLAKE. 1995. Habitat fragmentation in the temperate zone. Pages 357-380 in T.E. Martin and D. M. Finch eds., Ecology and management of neotropical migratory birds. Oxford University Press, New York and Oxford.

FENNEMAN, N.M. 1938. Physiography of the Eastern United States. McGraw-Hill, New York, New York, 714pp.

FINCH, D. M. 1991. Population ecology, habitat requirements, and conservation of neotropical migratory birds. U.S. For. Serv. Gen. Tech. Rep. RM-205.

HAGAN, J.M., III, P.S. MCKINLEY, A.L. MEEHAN, AND A.L. GROVE. 1997. Diversity and abundance of landbirds in a northeastern industrial forest. Journal of Wildlife Management 61: 718-735.

HAGAN, J.M.,III, W.M. VANDER HAEGEN, AND P.S. MCKINLEY. 1996. The early development of forest fragmentation effects on birds. Conservation Biology 10:188-202.

HARPOLE, D. N. AND C. A. HAAS. 1999. Effects of seven silvicultural treatments on terrestrial salamanders. Forest Ecology and Management 114:349-56.

HARRIS, L.D. 1984. The fragmented forest. Univ. of Chicago Press, Chicago, Illinois.

HARTLEY, M., AND M.L. HUNTER, Jr. 1998. A meta-analysis of forest cover, edge effects, and artificial nest predation rates. Conservation Biology 12:465-469.

HUNTER, M. L., JR. 1990. Wildlife, forests, and forestry: principles of managing forests for biological diversity. Prentice-Hall, Englewood Cliffs, New Jersey. 370pp. 
JAMES, F. C. 1971. Ordination of habitat relationships among breeding birds. Wilson Bulletin $83: 215-236$.

JAMES, F.C., AND H.H. SHUGART. 1970. A quantitative method of habitat description. Audubon Field Notes 24: 727-736.

JOHNSON, D. H.. 1999. The insignificance of statistical significance testing. Journal of Wildlife Management 63: 763-772.

MARTIN, T.E., C. PAINE, C.J. CONWAY, W. M. HOCHACHKA, P. ALLEN, and W. JENKINS. 1997. BBIRD Field Protocol. USGS, Biological Resources Division, Montana Cooperative Fish and Wildlife Research Unit, Missoula, Montana.

MACARTHUR, R. H., AND J. W. MACARTHUR. 1961. On bird species diversity. Ecology 50:594-598.

MCGARIGAL, K., AND B. J. MARKS. 1995. FRAGSTATS: spatial pattern analysis program for quantifying landscape structure. U. S. For. Serv. Gen. Tech. Rept. PNW -351.

MILLER, G.W. 1993. Financial aspects of partial cutting practices in central Appalachian hardwoods. U.S. For. Serv. Res. Pap. NE-673.

NETER, J., M.H. KUTNER, C. J. NACHTSHEIM, AND W. WASSERMAN. 1996. Applied Linear Statistical Models. Irwin. Homewood, Illinois. 1181pp.

NICHOLS, J.V. 1996. Effects of two-age timber management and clearcutting on songbird density and reproductive success. M.S. Thesis, West Virginia University. 131pp.

NUR, N., S. L. JONES, AND G. R. R GEUPEL 1999. A statistical guide to data analysis of avian monitoring programs. U.S. Fish and Wildl. Serv. Biol. Tech. Publ. BTP-R60011999.

PATON, W. 1994. The effect of edge on avian nest success: how strong is the evidence? Conservation Biology 8:17-26.

PETIT, L.J., D.R. PETIT, and T.E. MARTIN. 1995. Landscape-level management of migratory birds - looking past the trees to see the forest. Wildlife Society Bulletin 23:420-429.

RALPH, C.J., G.R. GUEPEL, P. PYLE, T.E. MARTIN, AND D.F. DESANTE. 1993. Handbook of field methods for monitoring landbirds. U.S. For. Serv. Gen. Tech. Rept. PSW-GTR-144. 
ROBINSON, S.K., F.R. THOMPSON III, T.M. DONOVAN, D.R. WHITEHEAD, AND J.

FAABORG. 1995. Regional forest fragmentation and the nesting success of migratory birds. Science 267:1987-1990.

$\mathrm{SAAB}, \mathrm{V}$. 1999. Importance of spatial scale to habitat use by breeding birds in riparian forests: a hierarchical analysis. Ecological Applications 9:135-151.

SAS INSTITUTE INC. 1991. SAS/STAT Guide for personal computers, version 6 edition. SAS Institute, Inc. Cary, N. Carolina. 1028pp.

SMITH, K. 1977. Distribution of summer birds along a forest moisture gradient in an Ozark watershed. Ecology 58:810-819.

STOKES, M. A., C. S. DAVIS, AND G. G. KOCH. 1995. Categorical Data Analysis Using the SAS System. Sas Institute Inc., Cary, North Carolina.

STRAUGHSBAUGH, P.D., AND E. L. CORE. 1977. Flora of West Virginia. Seneca Books, Grantsville, West Virginia.

THOMPSON, F. R., III, W. D. DIJAK, T. G. KULOWIEC, AND D A. HAMILTON. 1992.

Breeding bird populations in Missouri Ozark Forests with and without clearcutting. Journal of Wildlife Managemet 56:23-30.

THOMPSON, F.R., III, J.R. PROBST, AND M.G. RAPHAEL. 1995. Impacts of silviculture: overview and management recommendations. Pages 201-219 in T. E. Martin and D. M. Finch,eds., Ecology and management of neotropical migratory birds. Oxford University Press, New York and Oxford.

VILLARD, M., M. K. TRZCINSKI, AND G. MERRIAM. 1999. Fragmentation effects on forest birds: relative influence of woodland cover and configuration on landscape occupancy. Conservation Biology 13:774-783.

WHITMORE, R. C. 1979. Short-term change in vegetation structure and its effect on Grasshopper Sparrows in West Virginia. Auk 96: 621-625.

WILLSON, M. F. 1974. Avian community organization and habitat structure. Ecology 55:1017-1029.

ZAR, J. H. 1999. Biostatistical analysis, $4^{\text {th }}$ edition. Prentice Hall, Upper Saddle River, New Jersey. 663pp. 
Table 4.1. Weights used to calculate the contrast-weighted edge density among all possible pairs of edges on the Westvaco Wildlife and Ecosystem Research Forest, Randolph County, West Virginia, 1996-1998.

\begin{tabular}{lc}
\hline Pair & Weight \\
\hline Hardwood-Mixedwood & 0.00 \\
Hardwood-Diameter-limit & 0.25 \\
Hardwood-Regeneration & 1.00 \\
Hardwood-Open & 1.00 \\
Mixedwood-Diameter-limit & 0.25 \\
Mixedwood-Regeneration & 1.00 \\
Mixedwood-Open & 1.00 \\
Diameter-limit-Regeneration & 0.75 \\
Diameter-limit-Open & 0.75 \\
Regeneration-Open & 0.00 \\
\hline
\end{tabular}


Table 4.2. Presence of songbird species in fragmented $(n=212)$ and nonfragmented $(n=72)$ landscapes on the Westvaco Wildlife and Ecosystem Research Forest, Randolph County, West Virginia, 1996-1998.

\begin{tabular}{|c|c|c|c|c|c|c|}
\hline \multirow[b]{3}{*}{ Species } & \multicolumn{4}{|c|}{ Landscape } & \multirow[b]{3}{*}{$\begin{array}{c}\text { Cochran-Mantel- } \\
\text { Haenszel } \\
\text { Chi-square }\end{array}$} & \multirow[b]{3}{*}{$\mathrm{P}$} \\
\hline & \multicolumn{2}{|c|}{ Fragmented } & \multicolumn{2}{|c|}{ Nonfragmented } & & \\
\hline & $\begin{array}{l}\text { Points } \\
\text { Species } \\
\text { Present }\end{array}$ & $\begin{array}{c}\text { Proportion } \\
\text { of } \\
\text { Points } \\
\end{array}$ & $\begin{array}{l}\text { Points } \\
\text { Species } \\
\text { Present }\end{array}$ & $\begin{array}{c}\text { Proportion } \\
\text { of } \\
\text { Points } \\
\end{array}$ & & \\
\hline Edge-interior Species: & & & & & & \\
\hline Black-capped chickadee & 90 & 0.42 & 28 & 0.39 & 0.28 & 0.60 \\
\hline Dark-eyed junco & 143 & 0.67 & 42 & 0.58 & 1.91 & 0.17 \\
\hline Eastern towhee & 54 & 0.25 & 19 & 0.26 & 0.01 & 0.90 \\
\hline Rose-breasted grosbeak & 52 & 0.24 & 18 & 0.25 & $<0.01$ & 0.98 \\
\hline Forest-interior Species: & & & & & & \\
\hline Black-throated blue warbler & 86 & 0.41 & 40 & 0.56 & 5.10 & 0.02 \\
\hline Canada warbler & 113 & 0.53 & 39 & 0.54 & 1.45 & 0.23 \\
\hline Magnolia warbler & 87 & 0.41 & 22 & 0.31 & 2.28 & 0.13 \\
\hline Ovenbird & 59 & 0.28 & 26 & 0.36 & 1.76 & 0.18 \\
\hline Scarlet tanager & 122 & 0.57 & 35 & 0.49 & 1.57 & 0.21 \\
\hline Veery & 135 & 0.64 & 52 & 0.72 & 1.70 & 0.19 \\
\hline Winter wren & 108 & 0.51 & 35 & 0.49 & 0.13 & 0.72 \\
\hline
\end{tabular}


Table 4.3. Presence of songbird species in landscapes fragmented by diameter-limit harvests ( $\mathrm{n}=105)$, and by regeneration harvests $(\mathrm{n}=19)$, and in nonfragmented $(\mathrm{n}=159)$ landscapes on the Westvaco Wildlife and Ecosystem Research Forest, Randolph County, West Virginia, 1996-1998. Within a species, proportions with the same letter do not differ (pairwise Cochran-Mantel-Haenszel Chi-square, $\mathrm{P} \leq 0.03)$.

\begin{tabular}{|c|c|c|c|c|c|c|c|c|}
\hline \multirow[b]{3}{*}{ Species } & \multicolumn{6}{|c|}{ Landscape } & \multirow{3}{*}{$\begin{array}{c}\text { Omnibus } \\
\text { Cochran- } \\
\text { Mantel- } \\
\text { Haenszel } \\
\text { Chi-square } \\
\end{array}$} & \multirow[b]{3}{*}{$\mathrm{P}$} \\
\hline & \multicolumn{2}{|c|}{ Diameter-limit } & \multicolumn{2}{|c|}{ Regeneration } & \multicolumn{2}{|c|}{ Nonfragmented } & & \\
\hline & $\begin{array}{l}\text { Points } \\
\text { Species } \\
\text { Present }\end{array}$ & $\begin{array}{l}\text { Proportion } \\
\text { of Points }\end{array}$ & $\begin{array}{l}\text { Points } \\
\text { Species } \\
\text { Present }\end{array}$ & $\begin{array}{l}\text { Proportion } \\
\text { of Points }\end{array}$ & $\begin{array}{l}\text { Points } \\
\text { Species } \\
\text { Present }\end{array}$ & $\begin{array}{l}\text { Proportion } \\
\text { of Points }\end{array}$ & & \\
\hline Interior-edge Species: & & & & & & & & \\
\hline Black-capped chickadee & 48 & 0.46 & 7 & 0.37 & 62 & 0.39 & 1.34 & 0.51 \\
\hline Dark-eyed junco & 85 & $0.81 \mathrm{~A}$ & 8 & $0.42 \mathrm{~B}$ & 91 & $0.57 \mathrm{~A}$ & 9.86 & $<0.01$ \\
\hline Forest-interior Species: & & & & & & & & \\
\hline Black-throated blue warbler & 38 & $0.36 \mathrm{~B}$ & 14 & $0.74 \mathrm{~A}$ & 73 & $0.46 \mathrm{~B}$ & 44.93 & $<0.01$ \\
\hline Canada warbler & 71 & $0.67 \mathrm{~A}$ & 12 & $0.63 \mathrm{AB}$ & 87 & $0.55 \mathrm{~B}$ & 15.44 & 0.09 \\
\hline Ovenbird & 18 & $0.17 \mathrm{~B}$ & 10 & $0.53 \mathrm{~A}$ & 57 & $0.36 \mathrm{~A}$ & 8.28 & 0.01 \\
\hline Scarlet tanager & 47 & $0.45 \mathrm{~B}$ & 13 & $0.68 \mathrm{AB}$ & 106 & $0.67 \mathrm{~A}$ & 2.61 & 0.02 \\
\hline Veery & 69 & $0.66 \mathrm{AB}$ & 8 & $0.42 \mathrm{~B}$ & 109 & $0.68 \mathrm{~A}$ & 5.25 & 0.07 \\
\hline Winter wren & 68 & $0.65 \mathrm{~A}$ & 7 & $0.37 \mathrm{~B}$ & 58 & $0.36 \mathrm{~B}$ & 19.55 & $<0.01$ \\
\hline
\end{tabular}


Table 4.4. Means, standard errors (SE), minimum (Min) and maximum values (Max), and 95\% confidence coefficients (CI) for landscape variables on the Westvaco Wildlife and Ecosystem Research Forest, Randolph County, West Virginia, 1996-1998. Means with different letters differ among years (ANOVA, $\mathrm{P} \leq 0.10)$. Means are for untransformed data.

\begin{tabular}{|c|c|c|c|c|c|c|c|c|c|c|c|c|c|c|c|c|c|}
\hline \multirow[b]{2}{*}{ Variable } & \multirow[b]{2}{*}{$\mathrm{F}$} & \multirow[b]{2}{*}{ P-value } & \multicolumn{5}{|c|}{1996} & \multicolumn{5}{|c|}{1997} & \multicolumn{5}{|c|}{1998} \\
\hline & & & Mean & SE & Min & Max & CI & Mean & SE & Min & Max & CI & Mean & SE & Min & Max & CI \\
\hline CWED & 0.15 & 0.86 & 47.2 & 1.3 & 0.0 & 91.4 & 2.6 & 47.9 & 1.3 & 0.0 & 98.9 & 2.6 & 48.2 & 1.3 & 0.0 & 98.9 & 2.6 \\
\hline NP & 1.43 & 0.24 & 11.8 & 0.3 & 2.0 & 23.0 & 0.6 & 11.6 & 0.3 & 2.0 & 22.0 & 0.6 & 11.1 & 0.3 & 2.0 & 21.0 & 0.6 \\
\hline$\%$ Core & 9.41 & $<0.01$ & $39.6 \mathrm{~A}$ & 0.9 & 17.3 & 78.4 & 1.8 & $35.0 \mathrm{~B}$ & 1.0 & 4.9 & 78.4 & 2.1 & $33.4 \mathrm{~B}$ & 1.2 & 1.9 & 78.4 & 2.3 \\
\hline$\%$ Hard & 42.93 & $<0.01$ & $82.1 \mathrm{~A}$ & 0.6 & 53.5 & 98.9 & 1.1 & $73.0 \mathrm{~B}$ & 1.0 & 29.8 & 98.9 & 2.0 & $68.3 \mathrm{C}$ & 1.5 & 17.4 & 98.9 & 2.9 \\
\hline$\%$ Mix & 0.01 & 0.99 & 10.2 & 0.5 & 0.1 & 28.4 & 0.9 & 10.2 & 0.5 & 0.1 & 28.4 & 0.9 & 10.1 & 0.5 & 0.1 & 28.4 & 0.9 \\
\hline$\%$ Diam & 36.51 & $<0.01$ & $0.9 \mathrm{C}$ & 0.2 & 0.0 & 10.4 & 0.3 & $8.0 \mathrm{~B}$ & 0.9 & 0.0 & 50.2 & 1.8 & $12.7 \mathrm{C}$ & 1.4 & 0.0 & 66.9 & 2.8 \\
\hline$\%$ Regen & 14.96 & $<0.01$ & $1.9 \mathrm{~B}$ & 0.2 & 0.0 & 16.0 & 0.5 & $3.8 \mathrm{~A}$ & 0.3 & 0.0 & 17.5 & 0.6 & $4.0 \mathrm{~A}$ & 0.3 & 0.0 & 17.5 & 0.6 \\
\hline$\%$ Open & 0.00 & 1.00 & 4.9 & 0.3 & 0.0 & 17.5 & 0.6 & 4.9 & 0.3 & 0.0 & 17.5 & 0.6 & 4.9 & 0.3 & 0.0 & 17.5 & 0.6 \\
\hline
\end{tabular}


Table 4.5. Significant landscape variables selected by stepwise logistic regression for predicting the presence of songbird species on the Westvaco Wildlife and Ecosystem Research Forest, Randolph County, West Virginia, 1996-1998.

\begin{tabular}{|c|c|c|c|c|c|c|c|c|c|c|c|}
\hline \multirow[b]{2}{*}{ Species } & \multirow[b]{2}{*}{ Prop. $^{\text {a }}$} & \multicolumn{2}{|c|}{$\begin{array}{c}\text { Goodness of } \\
\text { Fit Test }^{\mathrm{b}}\end{array}$} & \multicolumn{8}{|c|}{ Landscape Variables $^{c}$} \\
\hline & & $\mathrm{X}^{2}$ & $\mathrm{P}$ & CWED & NP & $\%$ Core & $\%$ Hard & $\%$ Mix & $\%$ Diam & \%Regen & \%Open \\
\hline \multicolumn{12}{|l|}{ Interior-edge: } \\
\hline Black-capped chickadee & 0.41 & 9.07 & 0.34 & $\begin{array}{c}- \\
(1)\end{array}$ & & & & & & & \\
\hline Dark-eyed junco & 0.61 & 5.32 & 0.72 & - & & $\frac{-}{(3)}$ & $\begin{array}{l}+ \\
(4)\end{array}$ & & $\begin{array}{c}+ \\
(1)\end{array}$ & & \\
\hline Eastern towhee & 0.28 & 7.38 & 0.50 & & & (2) & & & & $\begin{array}{c}+ \\
(1)\end{array}$ & \\
\hline \multicolumn{12}{|l|}{ Forest interior: } \\
\hline Black-throated blue warbler & 0.44 & 6.48 & 0.59 & & & & & $\begin{array}{c}+ \\
(1)\end{array}$ & & $\begin{array}{l}+ \\
(2)\end{array}$ & $\frac{-}{(3)}$ \\
\hline Ovenbird & 0.32 & 5.75 & 0.67 & & & $\begin{array}{c}+ \\
(1)\end{array}$ & & & & $\begin{array}{l}+ \\
(2)\end{array}$ & $\begin{array}{l}+ \\
(3)\end{array}$ \\
\hline Scarlet tanager & 0.55 & 6.42 & 0.38 & & & & & & & $\begin{array}{l}+ \\
(1)\end{array}$ & \\
\hline Winter wren & 0.44 & 5.47 & 0.71 & & & & & & $\begin{array}{c}+ \\
(1)\end{array}$ & $(2)$ & \\
\hline
\end{tabular}

${ }^{\text {a }}$ Proportion of landscapes in which the species was detected during point count surveys.

${ }^{\mathrm{b}}$ Hosmer and Lemeshow goodness-of-fit test.

${ }^{c}$ Cells with a '+' or '-' indicate a significant relationship (Liklihood-ratio test: $\mathrm{P} \leq 0.10$ ). Numbers in parentheses indicate order entered into model. 
Table 4.6. Means, standard errors (SE), minimum (Min) and maximum values (Max), and 95\% confidence coefficients (CI) for microhabitat and landscape variables used in microhabitat and combined logistic regression models on the Westvaco Wildlife and Ecosystem Research Forest, Randolph County, West Virginia, 1996-1998. Means are for untransformed data.

\begin{tabular}{|c|c|c|c|c|c|c|c|c|c|c|c|c|}
\hline \multirow[b]{2}{*}{ Variables } & \multirow[b]{2}{*}{$\mathrm{F}$} & \multirow[b]{2}{*}{ P-value } & \multicolumn{5}{|c|}{1996} & \multicolumn{5}{|c|}{1998} \\
\hline & & & Mean & $\mathrm{SE}$ & Min & $\operatorname{Max}$ & $\mathrm{CI}$ & Mean & $\mathrm{SE}$ & Min & $\operatorname{Max}$ & $\mathrm{CI}$ \\
\hline \multicolumn{13}{|l|}{ Microhabitat: } \\
\hline Trees $>8-29.9 \mathrm{~cm} / 0.04-\mathrm{ha}$ & 0.73 & 0.40 & 14.8 & 0.6 & 6.0 & 27.5 & 1.2 & 13.98 & 0.8 & 0.0 & 27.7 & 1.5 \\
\hline Trees $\geq 30 \mathrm{~cm} / 0.04$-ha & 1.05 & 0.31 & 5.4 & 0.2 & 1.7 & 11.7 & 0.5 & 5.06 & 0.3 & 0.0 & 11.7 & 0.5 \\
\hline Understory Stems/0.008-ha & 0.04 & 0.84 & 48.0 & 6.3 & 2.5 & 406.0 & 12.6 & 49.85 & 6.4 & 1.2 & 406.0 & 12.7 \\
\hline VSI & 3.73 & 0.06 & 10.7 & 0.2 & 6.0 & 14.0 & 0.4 & 9.97 & 0.3 & 0.7 & 14.7 & 0.6 \\
\hline$\%$ Green & 0.82 & 0.37 & 25.2 & 1.7 & 0.0 & 65.0 & 3.4 & 27.40 & 1.7 & 0.0 & 65.0 & 3.4 \\
\hline$\%$ Litter & 4.23 & 0.04 & 58.9 & 2.0 & 21.2 & 91.2 & 4.0 & 52.71 & 2.2 & 5.0 & 87.5 & 4.5 \\
\hline$\%$ Wood & 1.31 & 0.25 & 7.7 & 0.6 & 0.0 & 22.5 & 1.1 & 8.66 & 0.7 & 0.0 & 30.0 & 1.3 \\
\hline$\%$ Bare & 2.45 & 0.12 & 7.9 & 0.9 & 0.0 & 33.7 & 1.8 & 10.09 & 1.1 & 0.0 & 43.7 & 2.2 \\
\hline \multicolumn{13}{|l|}{ Landscape: } \\
\hline CWED & 0.06 & 0.81 & 49.3 & 1.5 & 27.3 & 68.8 & 2.9 & 49.28 & 1.4 & 29.0 & 72.9 & 2.8 \\
\hline NP & 1.85 & 0.17 & 12.7 & 0.4 & 7.0 & 21.0 & 0.8 & 11.97 & 0.4 & 7.0 & 21.0 & 0.8 \\
\hline$\%$ Core & 7.19 & $<0.01$ & 36.8 & 1.2 & 17.3 & 53.4 & 2.3 & 32.12 & 1.4 & 7.6 & 52.1 & 2.9 \\
\hline$\%$ Hard & 41.25 & $<0.01$ & 81.3 & 0.6 & 70.3 & 92.1 & 1.1 & 68.20 & 2.0 & 27.8 & 86.0 & 3.9 \\
\hline
\end{tabular}


Table 4.6. Continued.

\begin{tabular}{|c|c|c|c|c|c|c|c|c|c|c|c|c|}
\hline \multirow[b]{2}{*}{ Variables } & \multirow[b]{2}{*}{$\mathrm{F}$} & \multirow[b]{2}{*}{ P-value } & \multicolumn{5}{|c|}{1996} & \multicolumn{5}{|c|}{1998} \\
\hline & & & Mean & $\mathrm{SE}$ & Min & $\operatorname{Max}$ & $\mathrm{CI}$ & Mean & $\mathrm{SE}$ & Min & $\operatorname{Max}$ & $\mathrm{CI}$ \\
\hline$\%$ Mix & 0.01 & 0.91 & 11.3 & 0.5 & 2.6 & 21.7 & 1.0 & 11.38 & 0.5 & 2.6 & 21.7 & 1.0 \\
\hline$\%$ Diam & 29.48 & $<0.01$ & 1.4 & 0.3 & 0.0 & 10.4 & 0.5 & 11.91 & 1.9 & 0.0 & 54.9 & 3.9 \\
\hline$\% \operatorname{Reg}$ & 18.99 & $<0.01$ & 1.9 & 0.3 & 0.0 & 12.8 & 0.6 & 4.46 & 0.5 & 0.0 & 17.5 & 1.0 \\
\hline$\%$ Open & 0.00 & 1.00 & 4.1 & 0.2 & 2.0 & 7.2 & 0.3 & 4.06 & 0.2 & 2.0 & 7.2 & 0.3 \\
\hline
\end{tabular}


Table 4.7. Microhabitat variables selected by stepwise logistic regression to predict the presence of interior-edge and forest-interior species on the Westvaco Wildlife and Ecosystem Research Forest, Randolph County, West Virginia, 1996-1998.

\begin{tabular}{|c|c|c|c|c|c|c|c|c|c|c|c|}
\hline \multirow[b]{2}{*}{ Species } & \multirow[b]{2}{*}{ Prop. $^{\text {a }}$} & \multicolumn{2}{|c|}{$\begin{array}{l}\text { Goodness of } \\
\text { Fit Test }^{b}\end{array}$} & \multicolumn{8}{|c|}{ Microhabitat Variables ${ }^{\mathrm{c}}$} \\
\hline & & $\mathrm{X}^{2}$ & $\mathrm{P}$ & Stree & LTree & Under & Vertd & Green & Litt & Wood & Bare \\
\hline \multicolumn{12}{|l|}{ Interior-edge: } \\
\hline$\overline{\text { Dark-eyed Junco }}$ & 0.27 & 4.47 & 0.81 & & & $\begin{array}{l}- \\
(1)\end{array}$ & & $\begin{array}{l}+ \\
(2)\end{array}$ & & & \\
\hline Eastern Towhee & 0.30 & 11.24 & 0.19 & & & & & & $\begin{array}{c}+ \\
(1)\end{array}$ & & \\
\hline \multicolumn{12}{|l|}{ Forest interior: } \\
\hline $\begin{array}{l}\text { Black-throated Blue } \\
\text { Warbler }\end{array}$ & 0.57 & 4.27 & 0.83 & $\frac{-}{(2)}$ & & & & & & & $(\overline{1)}$ \\
\hline Canada warbler & 0.62 & 5.30 & 0.62 & & & & & & $\begin{array}{l}- \\
(1)\end{array}$ & & \\
\hline Magnolia warbler & 0.47 & 12.14 & 0.14 & & & $\begin{array}{l}- \\
(1)\end{array}$ & & & & & \\
\hline Veery & 0.75 & 5.84 & 0.56 & & & & & & & $\begin{array}{c}+ \\
(1) \\
\end{array}$ & \\
\hline
\end{tabular}

${ }^{\text {a }}$ Proportion of point counts in which species were detected.

${ }^{\mathrm{b}}$ Hosmer and Lemeshow goodness-of-fit test.

${ }^{c}$ Cells with a '+' or '-' indicate a significant relationship (Likelihood-ratio test: $\mathrm{P} \leq 0.10$ ). Numbers in parentheses indicate order entered into model. 
Table 4.8. Combined landscape and microhabitat variables chosen by stepwise logistic regression to predict the presence of songbirds in 1996 and 1998 on the Westvaco Wildlife and Ecosystem Research Forest, Randolph County, West Virginia (n=69 each year).

\begin{tabular}{|c|c|c|c|}
\hline \multirow[b]{2}{*}{ Species } & \multicolumn{2}{|c|}{$\begin{array}{l}\text { Goodness of } \\
\text { Fit Test }\end{array}$} & \multirow[b]{2}{*}{ Models ${ }^{\mathrm{b}}$} \\
\hline & $\mathrm{X}^{2}$ & $\mathrm{P}$ & \\
\hline \multicolumn{4}{|l|}{ Interior-edge: } \\
\hline Dark-eyed junco & 10.38 & 0.24 & 14.87-0.13CWED -17.02\%Core -2.93Litt \\
\hline Eastern towhee & 8.20 & 0.41 & 2.52-4.13Litt-0.993 Year + 6.15\%Regen \\
\hline \multicolumn{4}{|l|}{ Forest interior: } \\
\hline Black-throated blue warbler & 7.16 & 0.52 & $-0.799+8.29 \%$ Regen-0.10Bare+0.03Green \\
\hline Canada warbler & 6.70 & 0.57 & $7.35-3.62 \mathrm{Litt}+4.12 \mathrm{NP}$ \\
\hline Magnolia warbler & 5.55 & 0.70 & 7.78-12.05\%Core +5.37Stree-6.40NP -11.14Wood -44.51\%Open \\
\hline Ovenbird & 6.50 & 0.59 & $-2.85-12.67$ Bare+19.43\% Core-7.74\%Hard+0.06CWED \\
\hline Scarlet tanager & 5.92 & 0.66 & $-0.86+27.47 \%$ Open \\
\hline Veery & 5.84 & 0.56 & $0.33+9.95$ Wood \\
\hline Winter wren & 6.97 & 0.22 & $-0.68+6.17 \%$ Diam \\
\hline
\end{tabular}

${ }^{\text {a }}$ Hosmer-Lemeshow goodness-of-fit test.

${ }^{\mathrm{b}}$ Variables are listed in order of importance based on entry into the model and Wald chi-square statistics. 
Table 4.9. Best-fit models predicting songbird occurrence on the Westvaco Wildlife and Ecosystem Research Forest, Randolph County, West Virginia, 1996-1998. Models with better performance are marked with an " $\mathrm{X}$ ".

\begin{tabular}{lccc}
\hline \multirow{2}{*}{ Species } & \multicolumn{3}{c}{ Model } \\
\cline { 2 - 3 } Edge-interior species: & Landscape & Microhabitat & Combined \\
\hline Black-capped chickadee & $\mathrm{X}$ & \\
Dark-eyed junco & & $\mathrm{X}$ \\
Eastern towhee & $\mathrm{X}$ & \\
Forest interior: & & $\mathrm{X}$ \\
Black-throated blue warbler & & $\mathrm{X}$ \\
Canada warbler & & \\
Magnolia warbler & & \\
Ovenbird & $\mathrm{X}$ & \\
Scarlet tanager & $\mathrm{X}$ & \\
Veery $^{\mathrm{b}}$ & & $\mathrm{X}$ \\
Winter wren $^{\mathrm{a}}$ & $\mathrm{X}$ & \\
\hline
\end{tabular}

${ }^{a}$ Combined model only had landscape variables.

${ }^{\mathrm{b}}$ Combined model only had microhabitat variables. 
Table 4.10. Wald chi-square test statistics and significance levels for variables selected in combined landscape and microhabitat models predicting bird species occurrences on the Westvaco Wildlife and Ecosystem Research Forest, Randolph County, West Virginia, 1996-1998.

\begin{tabular}{|c|c|c|c|c|c|c|c|c|c|}
\hline \multirow[b]{3}{*}{ Variable } & \multicolumn{9}{|c|}{$\begin{array}{c}\text { Wald Chi-square Statistic } \\
\text { (P-value) }\end{array}$} \\
\hline & \multicolumn{9}{|c|}{ Species $^{\mathrm{a}}$} \\
\hline & DEJU & EATO & BTBW & CAWA & MAWA & OVEN & SCTA & VEER & WIWR \\
\hline \multicolumn{10}{|l|}{ Microhabitat: } \\
\hline Trees $>8-29.9 \mathrm{~cm} / 0.04-\mathrm{ha}$ & & & & & $\begin{array}{c}11.83 \\
(<0.01)\end{array}$ & & & & \\
\hline \multicolumn{10}{|l|}{ Trees $\geq 30 \mathrm{~cm} / 0.04$-ha } \\
\hline \multicolumn{10}{|l|}{ Understory Stems/0.008-ha } \\
\hline \multicolumn{10}{|l|}{ VSI } \\
\hline$\%$ Green & & & $\begin{array}{c}5.09 \\
(0.02)\end{array}$ & & & & & & \\
\hline$\%$ Litter & $\begin{array}{l}7.03 \\
(0.01)\end{array}$ & $\begin{array}{l}14.22 \\
(<0.01)\end{array}$ & & $\begin{array}{c}13.27 \\
(<0.01)\end{array}$ & & & & & \\
\hline$\%$ Wood & & & & & $\begin{array}{c}6.20 \\
(0.01)\end{array}$ & & & $\begin{array}{c}4.56 \\
(0.03)\end{array}$ & \\
\hline$\%$ Bare & & & $\begin{array}{c}15.19 \\
(<0.01)\end{array}$ & & & $\begin{array}{l}11.93 \\
(<0.01)\end{array}$ & & & \\
\hline \multicolumn{10}{|l|}{ Landscape: } \\
\hline$\overline{\text { CWED }}$ & $\begin{array}{c}23.95 \\
(<0.01)\end{array}$ & & & & $\begin{array}{c}24.12 \\
(<0.01)\end{array}$ & $\begin{array}{c}7.75 \\
(0.01)\end{array}$ & & & \\
\hline NP & & & & $\begin{array}{l}5.06 \\
(0.02)\end{array}$ & $\begin{array}{l}7.05 \\
(<0.01)\end{array}$ & & & & \\
\hline$\%$ Core & $\begin{array}{c}26.59 \\
(<0.01)\end{array}$ & & & & & $\begin{array}{c}18.27 \\
(<0.01)\end{array}$ & & & \\
\hline$\%$ Hard & & & & & & $\begin{array}{c}9.23 \\
(<0.01)\end{array}$ & & & \\
\hline \multicolumn{10}{|l|}{$\%$ Mix } \\
\hline$\%$ Diam & & & & & & & & & $\begin{array}{c}10.90 \\
(<0.01)\end{array}$ \\
\hline
\end{tabular}


Table 4. 10. Continued.

\begin{tabular}{|c|c|c|c|c|c|c|c|c|c|}
\hline \multirow[b]{3}{*}{ Variable } & \multicolumn{9}{|c|}{$\begin{array}{l}\text { Wald Chi-square Statistic } \\
\text { (P-value) }\end{array}$} \\
\hline & \multicolumn{9}{|c|}{ Species $^{\mathrm{a}}$} \\
\hline & $\overline{D E J U}$ & EATO & BTBW & CAWA & MAWA & OVEN & SCTA & VEER & WIWR \\
\hline \%Regen & & $\begin{array}{c}8.14 \\
(<0.01)\end{array}$ & $\begin{array}{l}17.73 \\
(<0.01)\end{array}$ & & & & & & \\
\hline \%Open & & & & & $\begin{array}{c}5.84 \\
(0.02)\end{array}$ & & $\begin{array}{c}5.00 \\
(0.03)\end{array}$ & & \\
\hline
\end{tabular}

${ }^{a}$ DEJU=dark-eyed junco; EATO=eastern towhee; BTBW=black-throated blue warbler; CAWA=Canada warbler; MAWA=magnolia warbler; OVEN=ovenbird; SCTA=scarlet tanager; VEER=veery; WIWR=winter wren. 

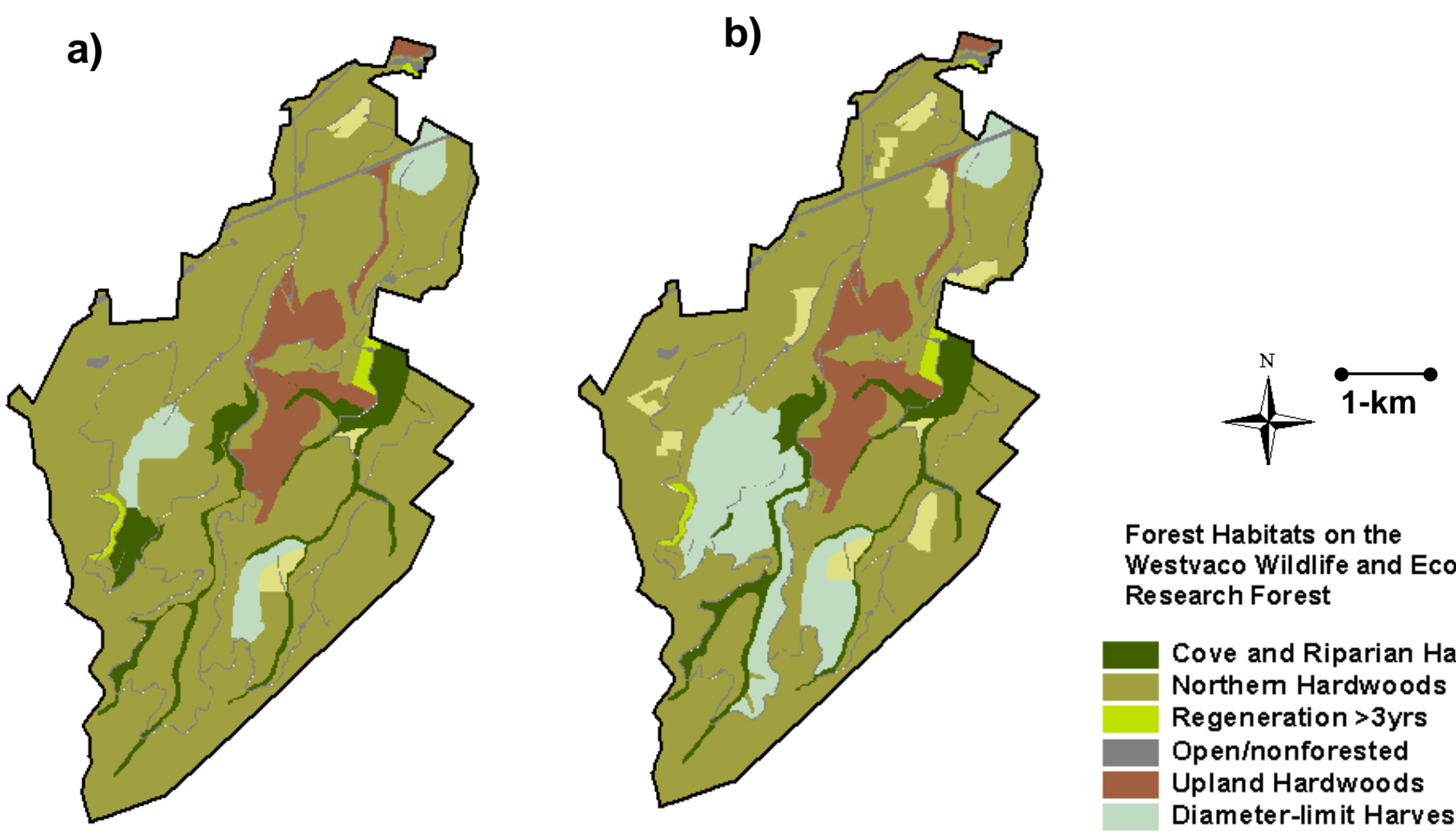

Forest Habitats on the

Westvaco Wildlife and Ecosystem

Research Forest

Cove and Riparian Hardwoods

Northem Hardwoods

Regeneration >3yrs

Open/nonforested

Upland Hardwoods

Diameter-lim it Harvest

Regeneration Harvest

Figure 4.1. Landscape composition of the Westvaco Wildlife and Ecosystem Research Forest, Randolph County, West Virginia in a) 1996 and b) 1998. 


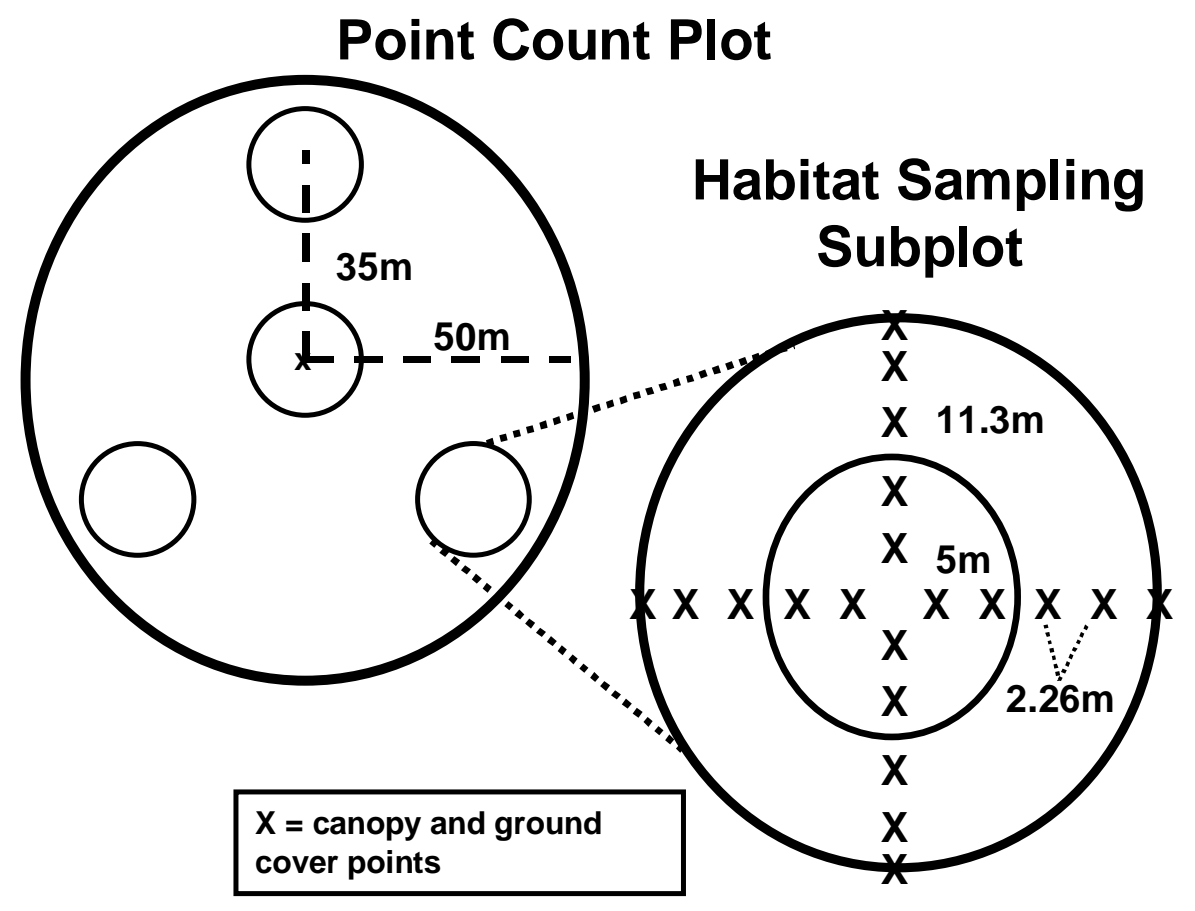

Figure 4.2. Layout-out of point count plots and habitat sampling subplots on the Westvaco Wildife and Ecosystem Research Forest, Randolph County, West Virginia, 1996-1998. 


\section{VITA}

\section{Cathy A. Weakland}

Cathy Ann Weakland was born the last of 6 children to Lawrence and Anna Weakland on October 26, 1969. She grew up in Euclid, Ohio, a suburb of Cleveland. She graduated from Euclid High School in 1988. She entered The Ohio State University in Columbus that same year and after 4 years received a Bachelor of Science degree in Zoology. She graduated magna cum laude with honors in zoology. It was at OSU where she developed a love for songbirds while studying nest defense and mate selection behavior of the hooded warbler.

After spending 4 months studying the endangered Kirtland's warbler in northern Michigan, she entered Purdue University in West Lafayette, Indiana to pursue a Master of Science degree in wildlife science, studying the effects of forest-road edges on neotropical migrant songbirds. She graduated in May of 1995. In January 1996 she began work on her doctoral dissertation research at West Virginia University under the guidance of Dr. Petra Bohall Wood. While at WVU she taught two courses, wildlife management techniques and wildlife management for foresters. She graduated in May 2000 with her Ph. D.

Although she has spent most of her adult life pursuing academic interests, she considers her greatest achievement to be her conversion to Christianity on December 13, 1995, 2 weeks prior to moving to Morgantown. However, she realizes that this really is not her achievement, but merely a result of the divine work of the Holy Spirit. Like the apostle Paul, she believes:

"I consider everything a loss compared to the surpassing greatness of knowing Christ Jesus my Lord...I consider them rubbish that I may gain Christ and be found in Him, not having a righteousness of my own that comes from law, but that which is through faith in Christ - the righteousness that comes from God and is by faith. I want to know Christ and the power of His resurrection and the fellowship of sharing in His sufferings, becoming like Him in his death, and so, somehow, to attain to the resurrection from the dead." Phil. 3: 8-11 (NIV)

- the apostle Paul of Tarsus, as a prisoner in Rome to the converts in Philippi ( 60 AD) 